GA-A 13752

UC-77

\title{
PROPERTIES OF UNIRRADIATED \\ FUEL ELEMENT GRAPHITES \\ H-451 AND TS-1240
}

\author{
by \\ W. R. JOHNSON \\ and G. B. ENGLE
}

- NOTICE - Mocount of work This report was prepared as an acorment. Neither sponsored by the United the United States Energy the United States nor Administration, nor any of Research and Dess nor any of their conkracts, their employes, or their employees, makes legat

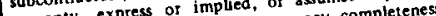
warkity or responsibility for the accuspatus, product or

Prepared under Contract E(04-3)-167

Project Agreement No. 17

for the San Francisco Operations Office

U.S. Energy Research and Development Administration 


\section{DISCLAIMER}

This report was prepared as an account of work sponsored by an agency of the United States Government. Neither the United States Government nor any agency Thereof, nor any of their employees, makes any warranty, express or implied, or assumes any legal liability or responsibility for the accuracy, completeness, or usefulness of any information, apparatus, product, or process disclosed, or represents that its use would not infringe privately owned rights. Reference herein to any specific commercial product, process, or service by trade name, trademark, manufacturer, or otherwise does not necessarily constitute or imply its endorsement, recommendation, or favoring by the United States Government or any agency thereof. The views and opinions of authors expressed herein do not necessarily state or reflect those of the United States Government or any agency thereof. 


\section{DISCLAIMER}

Portions of this document may be illegible in electronic image products. Images are produced from the best available original document. 


\section{ABSTRACT}

Nuclear graphite grades H-451 (Great Lakes Carbon Company) and TS-1240 (Union Carbide Corporation) are described and property data are presented for the unirradiated state. Properties measured included bulk density, ultimate tensile strength, modulus of elasticity, Poisson's ratio, thermal expansivity, and thermal conductivity. The data presented represent the minimum and maximum property values of the prototype full-size production logs and are indicative of property values to be expected in commercial production. 

CONTENTS

ABSTRACT . . . . . . . . . . . . . . . . . . $\quad$. $i i i$

1. SUMMARY . . . . . . . . . . . . . . . . . . . . 1

2. INTRODUCTION . . . . . . . . . . . . . . . . . . 3

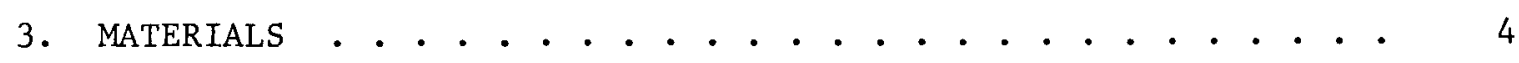

3.1. $\mathrm{H}-451$. . . . . . . . . . . . . . . . 4

3.2. TS 1240 . . . . . . . . . . . . . . . . 4

4. EXPERIMENTAL METHODS .................... . . . 7

5. EXPERIMENTAL RESULTS . . . . . . . . . . . . . . . 8

5.1. Sampling . . . . . . . . . . . . . . 8

5.2. Properties . . . . . . . . . . . . . . 8

5.2.1. Bulk Density ............. . 10

5.2.2. Tensile Properties . . . . . . . . . . 10

5.2.3. Thermal Expansivity . . . . . . . . . 19

5.2.4. Thermal Conductivity ............ 24

5.2.5. Impurity Content............. . 24

6. DISCUSSION AND CONCLUSIONS ............... . . . 28

6.1. Discussion . . . . . . . . . . . . . . 28

6.1.1. $\mathrm{H}-451$. . . . . . . . . . . . . . . 28

6.1.2. TS -1240 . . . . . . . . . . . . 29

6.2. Conclusions . . . . . . . . . . . . . . . 29

ACKNOWLEDGMENTS . . . . . . . . . . . . . . . . 30

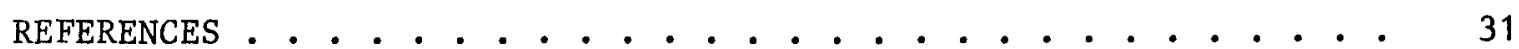

APPENDIX A. EXPERIMENTAL METHODS ............... A-1

APPENDIX B. TABULATED DATA ................ . . B-1 
5-1. Sampling plan of graphite $\log$ showing material designated

for property measurements .............. 9

5-2. Orientation of orthogonal axes for definition of

Poisson's ratio................... . 21

A-1. Cyclic stress-strain curve for graphite . . . . . . . A-2

TABLES

1-1. Summary of unirradiated properties, $\mathrm{H}-451$ and TS-1240

graphites . . . . . . . . . . . . . . . . 2

3-1. Description of $\mathrm{H}-451$ and TS-1240 graphites . . . . . . . 5

5-1. Bulk density: $\mathrm{H}-451$. . . . . . . . . . . 11

5-2. Bulk density: TS-1240, lot 1............ 12

5-3. Ultimate tensile strength: H-451 .......... 13

5-4. U1timate tensile strength: TS -1240 , lot 1 . . . . . . 14

5-5. Modulus of elasticity: H-451 . . . . . . . . . 16

5-6. Modulus of elasticity: TS-1240, lot 1 . . . . . . . 17

5-7. Poisson's ratio: $\mathrm{H}-451$ and $\mathrm{TS}-1240$.......... . 20

5-8. Thermal expansivity: $\mathrm{H}-451$. . . . . . . . . . 22

5-9. Thermal expansivity: TS-1240, lot 1 ......... 23

5-10. Thermal conductivity: $\mathrm{H}-451$. . . . . . . . . . 25

5-11. Thermal conductivity: TS-1240, lot 1 . . . . . . . . 26

5-12. Impurity content . . . . . . . . . . . . . . 27

$\mathrm{A}-1$. Losses resulting from ashing . . . . . . . . . . . . A-7

B-1. Density and tensile properties of $\mathrm{H}-451$, lot 266 ,

$\mathrm{B}-2$. Tensile properties of $\mathrm{H}-451$, $\operatorname{lot} 266, \log 5651-28$. . . B-4

$\mathrm{B}-3$. Density and tensile properties of $\mathrm{H}-451,1$ ot $266,10 \mathrm{~g}$ 5651-58 ................. . . B-5

B-4. Density and tensile strength of $\mathrm{H}-451$, lot $408, \log$ 5651-90, 0.25-in.-diameter by 0.9-in.-long specimen . . . B-6

B-5. Density and tensile strength of $\mathrm{H}-451$, lot $408,10 \mathrm{~g}$ 5651-90, 0.505-in.-diameter by 3.0-in.-long specimen . . B-14

$\mathrm{B}-6$. Density and tensile properties of $\mathrm{H}-451$, lot $408,10 \mathrm{~g}$ 5651-86, 0.505-in.-diameter by 3.0-in.-long specimen . . B-22 
TABLES (Continued)

B-7. Density and tensile properties of $\mathrm{H}-451$, lot 408 , log 5651-86, 0.25-in.-diameter by 0.9-in.-long specimen . . B-23

B-8. Density and tensile properties of H-451, lot 426, $\log 6484-33$. . . . . . . . . . . . . . B-25

B-9. Density and tensile properties of $\mathrm{H}-451$, lot 426, $\log 6484-34$. . . . . . . . . . . . . . . B-29

B-10. Density and tensile properties of H-451, lot 426, $\log 6484-40$. . . . . . . . . . . . . . B-33

$\mathrm{B}-11$. Density and tensile properties of $\mathrm{H}-451$, lot 426, $\log 6484-41$

$\mathrm{B}-12$. Density and tensile properties of TS-1240, lot 1 , $\log 5651-72$

B-13. Density and tensile properties of TS-1240, lot 1 , $\log 5651-73,0.505-i n .-d i a m e t e r$ by 3.0-in.-long specimens B-45

B-14. Density and tensile properties of TS-1240, lot 1, log 5651-73,0.25-in.-diameter by 0.9-in.-1ong specimens . B-46

B-15. Density and tensile properties of TS-1240, lot 1, $\log 5651-74$................ . B-48

B-16. Density and tensile properties of TS-1240, lot 1, $\log 5651-75$............... . . B-52

B-17. Density and tensile properties of TS-1240, lot 1 , $\log 6484-29$................... B-56

$\mathrm{B}-18$. Poisson's ratio: $\mathrm{H}-451$. . . . . . . . . B-61

B-19. Poisson's ratio: TS-1240 . . . . . . . . . . . $\mathrm{B}-69$

B-20. Thermal expansivity of $\mathrm{H}-451, \operatorname{lot} 408, \log 6484-24$. . . B-70

B-21. Thermal expansivity of $\mathrm{H}-451, \operatorname{lot} 408, \log 5651-86$. . . B-71

B-22. Thermal expansivity of $\mathrm{H}-451, \operatorname{lot} 426, \log 6484-33$. . . B-72

$\mathrm{B}-23$. Thermal expansivity of $\mathrm{H}-451, \operatorname{lot} 426, \log 6484-34$. . . B-74

B-24. Thermal expansivity of $\mathrm{H}-451, \operatorname{lot} 426, \log 6484-41$. . B B-76

B-25. Therma1 expansivity of TS-1240, lot 1, $\log 5651-72$. . B-78

B-26. Thermal expansivity of TS-1240, $\operatorname{lot} 1, \log 5651-73 . \cdots \mathrm{B}-80$

B-27. Thermal expansivity of TS-1240, $\operatorname{lot} 1, \log 5651-74$. . B-81

B-28. Thermal expansivity of TS-1240, lot 1, $\log 5651-75$. . . B-83

B-29. Therma1 expansivity of TS-1240, $\operatorname{lot} 1, \log 6484-29 . .$. B-85 
$\mathrm{B}-30$. Thermal conductivity of $\mathrm{H}-451, \log 266, \log 5651-28$. . . B-87 $\mathrm{B}-31$. Thermal conductivity of $\mathrm{H}-451, \log 408, \log 5651-86$. . . B-88 $\mathrm{B}-32$. Thermal conductivity of $\mathrm{H}-451$, lot 426 , logs $6484-34$ and 6484-41................... . . B-89

B-33. Thermal conductivity of TS-1240, lot 1, logs 5651-72 and 5651-75................. . B-90

B-34. Thermal conductivity of TS-1240, $\operatorname{lot} 1, \log 5651-73$. . . B-91

B-35. Impurity content of H-451 . . . . . . . . . . . . B-92

$\mathrm{B}-36$. Impurity content of TS-1240, lot 1 . . . . . . . . B-95

B-37. Burnable and nonburnable boron equivalent values for $\mathrm{H}-451$ and TS-1240 . . . . . . . . . . . . . . B-97

B-38. Impurity contents of $\mathrm{H}-451$ and $\mathrm{TS}-1240$. . . . . . . . B-99

B-39. Spark mass spectrometric impurity analysis of $\mathrm{H}-451$ and TS-1240................. . . . B-100

B-40. Summary of lithium analyses for $\mathrm{H}-451$. . . . . . . . . B-101

B-41. Summary of lithium analyses for TS-1240 . . . . . . . . B-102 


\section{SUMMARY}

Nuclear graphite grades H-451 [Great Lakes Carbon Company (GLCC)] and TS-1240 [Carbon Products Division of Union Carbide Corporation (UCC)] are described and physical, mechanical, and chemical property data are presented on the graphites in the unirradiated state. Dimensional and property change data are given in Refs. 1 through 3.

A summary of the property data is given in Table 1-1. These data represent the minimum and maximum property values of the prototype graphites and indicate the property values to be expected in commercial production. Grade $\mathrm{H}-451$ has been selected for replacement fuel and reflector elements in the Fort St. Vrain High-Temperature Gas-Cooled Reactor. Commercial production of approximately 350 logs of Grade H-451 commenced in 1975 .

Grades TS-1240 and S0818 [Airco Speer Division of the Air Reduction Company (AS)] are also being evaluated for use in LHTGRs. 
TABLE $1-1$
SUMMARY OF UNIRRADIATED PROPERTIES, $\mathrm{H}-451^{\text {(a) }}$ AND TS-1240 GRAPHITES

\begin{tabular}{|c|c|c|c|c|}
\hline \multirow[b]{3}{*}{ Property } & \multicolumn{4}{|c|}{ Mean Value \pm Standard Deviation (Position In Log) } \\
\hline & \multicolumn{2}{|c|}{ Axial ${ }^{(b)}$} & \multicolumn{2}{|c|}{ Radial (b) } \\
\hline & Minimum & Maximum & Minimum & Maximum \\
\hline \multicolumn{5}{|l|}{ Log bulk density $\left(\mathrm{g} / \mathrm{cm}^{3}\right)$} \\
\hline $\mathrm{H}-451$ & $1.707 \pm 0.018$ & $1.739 \pm 0.019$ & -- & -- \\
\hline TS -1240 & $1.800 \pm 0.007$ & $1.808 \pm 0.013$ & -- & -- \\
\hline \multicolumn{5}{|c|}{ Ultimate tensile strength (psi) } \\
\hline H-451 & $1980 \pm 234$ (MLC) & $2758 \pm 269$ (MLE) & $1560 \pm 304$ (MLC) & $2208 \pm 326(\mathrm{EE})$ \\
\hline TS -1240 & $1990 \pm 415(\mathrm{EC})$ & $2446 \pm 368$ (MLE) & $1430 \pm 413(\mathrm{MLC})$ & $2090 \pm 263(E E)$ \\
\hline \multicolumn{5}{|c|}{ Modulus of elasticity $\left(10^{6}\right.$ psi) } \\
\hline $\mathrm{H}-451$ & $1.15 \pm 0.04$ (MLC) & $1.26 \pm 0.09$ (EE) & $1.00 \pm 0.04$ (MLC) & $1.08 \pm 0.06(\mathrm{EE})$ \\
\hline TS-1240 & $1.08 \pm 0.18(\mathrm{MLC})$ & $1.17 \pm 0.11$ (MLE) & $0.98 \pm 0.11$ (MLC) & $1.08 \pm 0.09(\mathrm{EC})$ \\
\hline \multicolumn{5}{|l|}{ Poisson's ratio } \\
\hline $\mathrm{H}-451$ & $0.110 \pm 0.010(\mathrm{EE})$ & $0.127 \pm 0.013(\mathrm{MLC})$ & $0.108 \pm 0.008$ (MLC) & $0.110 \pm 0.009(\mathrm{MLC})$ \\
\hline TS -1240 & \multicolumn{2}{|c|}{$0.152 \pm 0.010(Q L C)$} & \multicolumn{2}{|c|}{$0.129 \pm 0.007$ (QLC) } \\
\hline \multicolumn{3}{|c|}{ Thermal expansivity $\left(10^{-6}{ }^{\circ} \mathrm{C}^{-1}\right)$} & & \\
\hline $\mathrm{H}-451$ & $3.89 \pm 0.16$ (MLE) & $4.07 \pm 0.22(\mathrm{EC})$ & $4.52 \pm 0.23$ (MLC) & $4.63 \pm 0.17$ (EE) \\
\hline TS -1240 & $4.27 \pm 0.39(\mathrm{EC})$ & $4.48 \pm 0.25(\mathrm{EE})$ & $4.69 \pm 0.28$ (MLC) & $4.95 \pm 0.22(\mathrm{EE})$ \\
\hline Thermal conductivity, 800 & & & & \\
\hline $\mathrm{H}-451$ & $0.155=$ & $\pm 0.009(\mathrm{MLC})$ & $0.150 \pm 0$ & 010 (MLC) \\
\hline TS- 1240 & $0.148=$ & \pm 0.014 (MLC) & $0.147 \pm 0$ & 012 (MLC) \\
\hline
\end{tabular}

(a) Data for $10 t 426$ only.

(b) Direction of applied stress for Poisson's ratio. 


\section{INTRODUCTION}

During the past several years nuclear graphite development for the LHTGR in the U.S. has been directed toward producing near-isotropic graphites for fuel and replaceable reflector elements. The development effort has been concentrated on the use of petroleum-based near-isotropic cokes and conventional manufacturing processes. The development of the nearisotropic graphites has progressed to the state where full-size preproduction prototype logs have been manufactured in production equipment. Three near-isotropic graphite grades, one by each of the three major U.S. graphite manufacturers, are under development; grade H-451 by GLCC, grade TS- 1240 by UCC, and grade S0818 by AS. The above mentioned graphites are being evaluated by GA.

This report describes the results of property measurements on grades $\mathrm{H}-451$ and TS-1240 in the unirradiated state. Irradiation data on H-451 and TS-1240 are reported elsewhere (Refs. 1 through 3). Data are presented for three prototype lots of grade $\mathrm{H}-451$ and for one prototype lot of TS- 1240 . 


\section{MATERIALS}

Prototype lots of grades $\mathrm{H}-451$ and TS -1240 were manufactured by extrusion with petroleum-based near-isotropic cokes. The cokes are considered proprietary products of the respective manufacturers. Conventional manufacturing processes, similar to those used for the production of steel-furnace electrodes, were used. A description of graphites $\mathrm{H}-451$ and TS-1240 is given in Table $3-1$.

\section{3. $1 . \mathrm{H}-451$}

The prototype H-451 lots were manufactured with calcined cokes from different coke sources designated A and B. A single near-isotropic coke, designated $A$, was used to manufacture a 6-in.-diameter pilot plant grade $\mathrm{H}-419$, which was the initial development effort at GLCC leading to H-451. A description of $\mathrm{H}-429$ along with property and irradiation data was reported in Ref. 1. Coke A was also used to produce the first full-size prototype (lot 266) of H-451. Subsequent H-451 lots 408 and 426 were produced with a blend of coke A and a second coke designated $B$. The coke sources and data are considered to be proprietary by GLCC. Three different batches of coke from source A were used in the development program (see Table 3-1). Coal-tar pitch was used as the binder and petroleum pitch as an impregnant. One additional lot, 440, is under investigation at GA, but data were not available for this report. Lot 440 was manufactured with a blend of cokes $B$ and $C$. Lot 426 has been selected as the reference for future production; therefore, the data from lot 426 are of most importance in assessing $\mathrm{H}-451$ and are used to describe $\mathrm{H}-451$.

\subsection{TS -1240}

Grade TS-1240 was manufactured with a single-source near-isotropic calcined petroleum coke. The details of this coke are considered 
TABLE 3-1

DESCRIPTION OF H-451 AND TS-1240 GRAPHITES

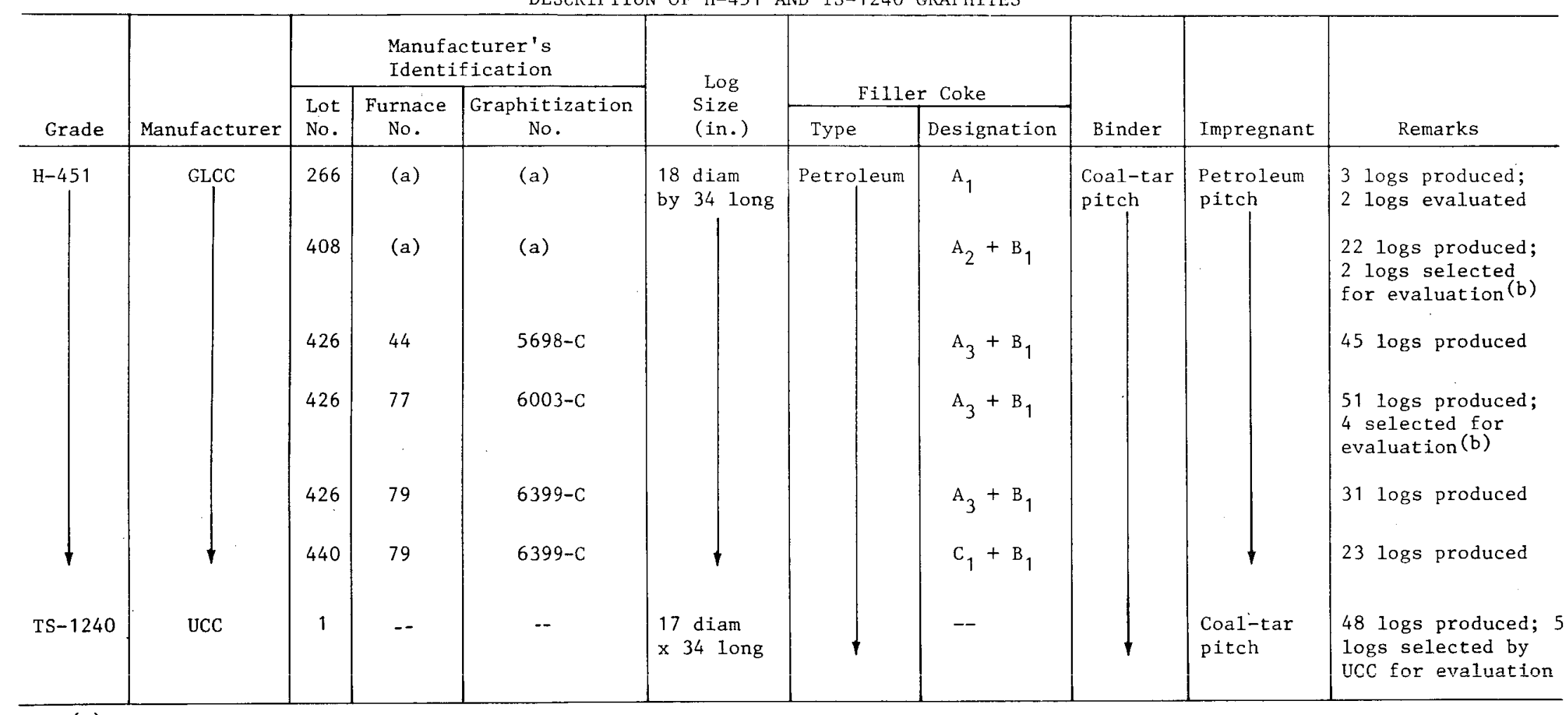

(a) Unknown to authors.

(b) Logs with a whole $\log$ density (measured by the manufacturer) equal to the mean density \pm one standard deviation of the logs in a given lot were selected for evaluation. 
proprietary by UCC. Coal-tar pitch was used as the binder and impregnant. A portion of the logs from the first batch of TS -1240 was graphitized as 34-in.-1ong logs while others were graphitized as 68-in.-long logs and halved to produce two standard 34-in.-long logs. 
4. EXPERIMENTAL METHODS

Details of the experimental methods are given in Appendix A. 


\section{EXPERIMENTAL RESULTS}

\subsection{SAMPLING}

Commercial graphite logs of the size used for fuel and reflector elements in HTGRs (17 in. in diameter by $34 \mathrm{in.} \mathrm{1ong)} \mathrm{have} \mathrm{been} \mathrm{shown} \mathrm{to}$ have a nonuniform distribution of properties within a single $10 \mathrm{~g}$ and from $\log$ to $\log$ within a manufactured lot (Refs. 1,4). Therefore, the properties and impurity contents were measured as a function of location within each $\log$ tested. Properties were measured on specimens taken paralle1 (axial) and perpendicular (radial) to the extrusion direction and at four different locations within the log: midlength center (MLC), end center (EC), midlength edge (MLE), and end edge (EE) (see Fig. 5-1). One $\log$ of TS-1240 [5651-72 (UCC 32)] was one-half of a 1 og that was 68 in. long during graphitization. This $10 g$ was sampled at a quarterlength center (QLC) 1ocation and a quarterlength edge (QLE) 1ocation in the 68-in. log. QLC and QLE of the 68-in. log are equivalent to MLC and MLE of the $34-i n$. $10 g$.

\subsection{PROPERTIES}

Complete data sets of the properties measured are given in Appendix B. The data are presented in summary form in this section.

The following properties were measured on $\mathrm{H}-451$ and $\mathrm{TS}-1240$ : (1) bulk density; (2) tensile properties including ultimate tensile strength, strain at fracture, modulus of elasticity, and Poisson's ratio; (3) thermal expansivity; (4) thermal conductivity; and (5) impurity content. 


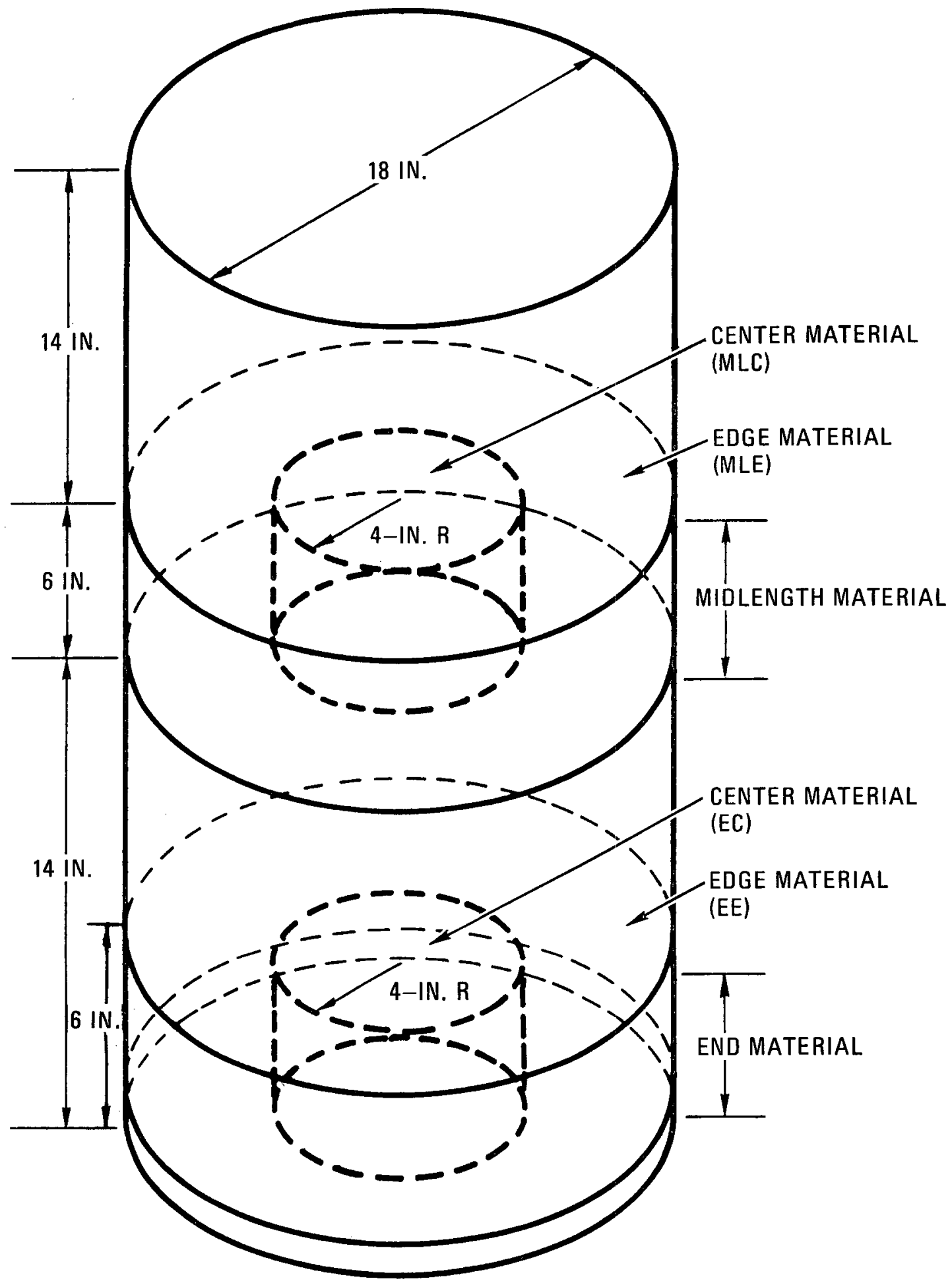

Fig. 5-1. Sampling plan of graphite log showing material designated for property measurements 


\subsubsection{Bulk Density}

Bulk density measurements were made on specimens 0.505 in. in diameter by 3.0 in. long unless otherwise noted. The bulk density data are summarized in Tables 5-1 and 5-2. The tables include calculated whole log density values obtained by averaging the mean values from the center and edge specimens. Proper weight was given in the calculations to the volume of the parent $\log$ that the small specimens represented. The bulk densities measured on whole logs in the manufacturer's plant were significantly higher than those measured at GA on small specimens throughout the $10 \mathrm{~g}$. Both graphites show a small but significant increase in density from the center to the edges of the log. However, several logs had no gradient. The density was, in general, lowest at the MLC position and increased slightly near the edges and ends of the logs. Again a few exceptions were observed. The variation in density for $\mathrm{H}-451$, 1ot 426, was 1.695

to $1.753 \mathrm{~g} / \mathrm{cm}^{3}$. The variation for TS-1240, lot 1 (excluding $\log 5651-72$ ), was 1.792 to $1.858 \mathrm{~g} / \mathrm{cm}^{3}$.

\subsubsection{Tensile Properties}

Tensile tests were conducted in air at room temperature on 0.505-in.diameter by 3.0-in.-long specimens or 0.25-in.-diameter by 0.90-in.-long specimens. Poisson's ratio measurements were conducted in air at room temperature on $0.50-i n$. by $0.50-i n$. by 6.0 -in.-long specimens or 0.505-in.diameter by 6.0-in.-1ong specimens at stresses up to 1200 psi.

\subsubsection{Ultimate Tensile Strength}

The ultimate tensile strength data are summarized in Tables 5-3 and 5-4. The mean ultimate tensile strength values of the 0.505- and 0.25-in.diameter specimens differed by only $5 \%$ in equivalent positions; therefore, the values from both sets of specimens were considered together. The tensile strength of $\mathrm{H}-451$ and $\mathrm{TS}-1240$ is highest in the axial direction, lowest at MLC for axial and radial specimens, and increases from the center to the edges. 
TABLE 5-

BULK DENSITY: H-451

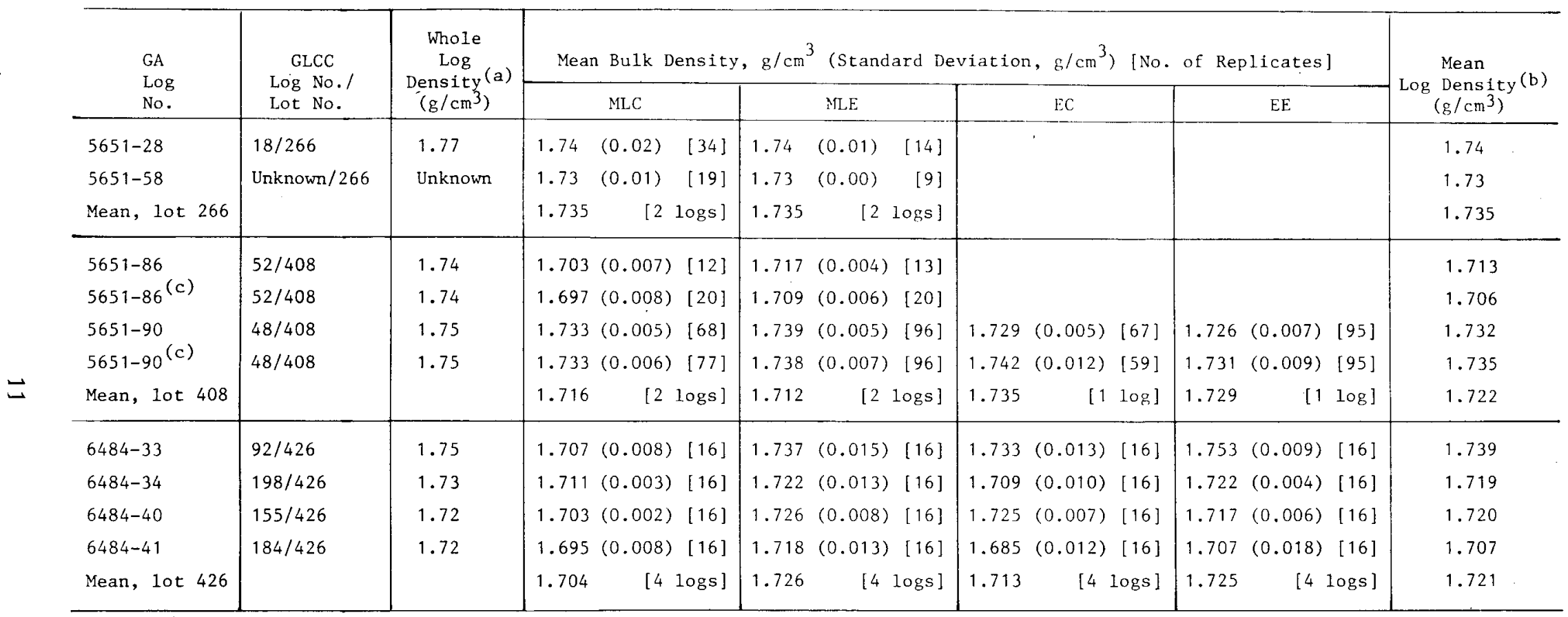

(a) Measured by GLCC.

(b) Calculated from small specimens.

(c) 0.25 -in.-diameter by 0.90-in.-1ong specimens. 
TABLE $5-2$
BULK DENSITY: TS-1240, LOT

\begin{tabular}{|c|c|c|c|c|c|c|c|c|c|}
\hline \multirow{2}{*}{$\begin{array}{l}\text { GA } \\
\text { Log } \\
\text { No. }\end{array}$} & \multirow{2}{*}{$\begin{array}{l}\text { UCC } \\
\text { Log } \\
\text { No. }\end{array}$} & \multirow{2}{*}{$\begin{array}{l}\text { Whole } \\
\text { Log } \\
\text { Density (a) } \\
\left(\mathrm{g} / \mathrm{cm}^{3}\right)\end{array}$} & \multicolumn{6}{|c|}{ Mean Bulk Density, $\mathrm{g} / \mathrm{cm}^{3}$ (Standard Deviation, $\mathrm{g} / \mathrm{cm}^{3}$ ) [No. of Replicates] } & \multirow{2}{*}{$\begin{array}{l}\text { Mean } \\
\text { Legsity }(b) \\
\left(g / \mathrm{cm}^{3}\right)\end{array}$} \\
\hline & & & MLC & MLE & QLC & QLE & EC & EE & \\
\hline $5651-72$ & 32 & 1.77 & & & $1.729(0.008)[17]$ & $1.750(0.014)[20]$ & $1.725(0.013)[17\}$ & $1.741(0.012)[18]$ & - \\
\hline $5651-73$ & 40 & 1.79 & $1.784(0.005)[12]$ & $1.805(0.006)[15]$ & & & & & 1.800 \\
\hline $5651-73^{(c)}$ & 40 & 1.79 & $1.773(0.006)[40]$ & $1.808(0.007)[40]$ & & & & & 1.800 \\
\hline $5651-74$ & 42 & 1.82 & $1.792(0.004)[16]$ & $1.803(0.006)[16]$ & & & $1.804(0.023)[17]$ & $1.806(0.014)[16]$ & 1.803 \\
\hline $5651-75$ & 46 & 1.84 & $1.812(0.006)[29]$ & $1.814(0.006) \cdot[20]$ & & & $1.815(0.004)[16]$ & $1.798(0.015) \quad[19]$ & 1.808 \\
\hline $6484-29$ & $\begin{array}{l}8-1 \mathrm{~S}- \\
\text { ID-23 }\end{array}$ & 1.83 & $1.795(0.003)[16]$ & $1.800(0.006)[18]$ & & & $1.802(0.004)[16]$ & $1.804(0.007)[19]$ & 1.800 \\
\hline Mean, lot 1 & & & {$\left[\begin{array}{ll}4 & \log 8\end{array}\right]$} & {$\left[\begin{array}{ll}4 & \log s\end{array}\right]$} & & & [ 3 logs] & [ 3 logs $]$ & 1.803 \\
\hline
\end{tabular}

(a) Measured at UCC.

(b) Calculated from small specimens.

(c) $0.25-$ in.-diameter by $0.90-$ in.-1ong specimens. 
TABLE 5-3

ULTIMATE TENSILE STRENGTH: H-451

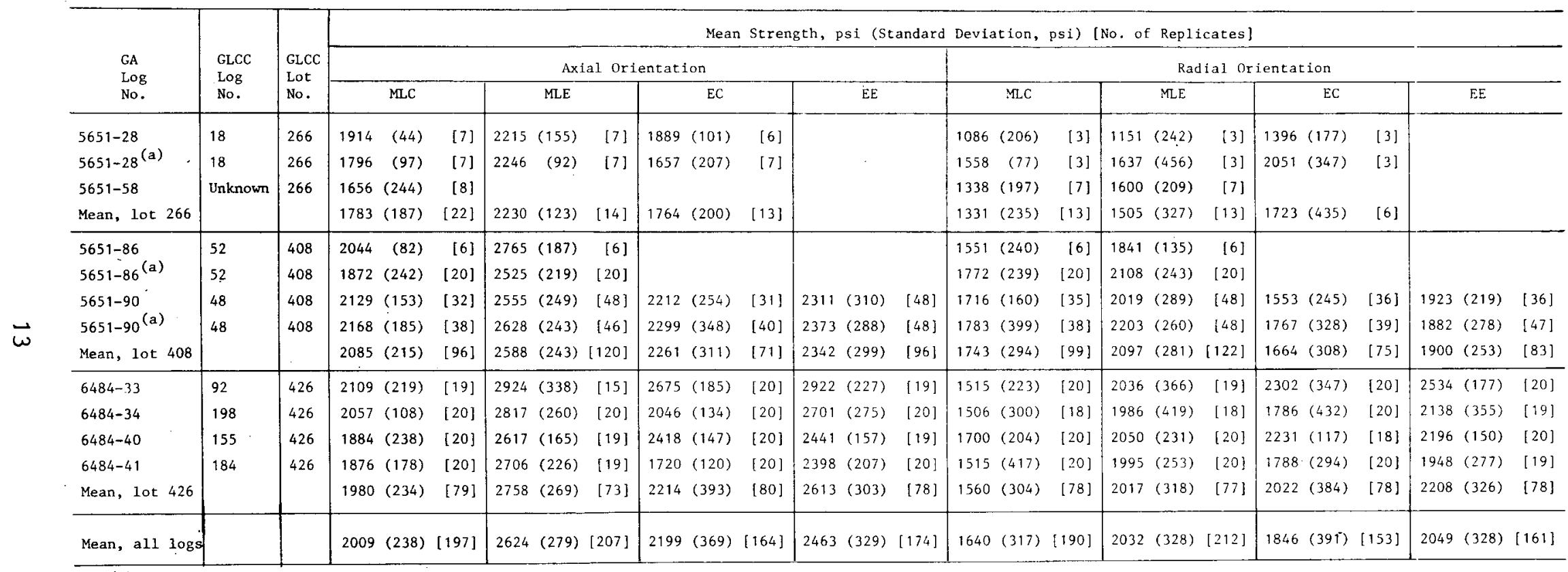

(a) Specimens were $0.25 \mathrm{in.}$ in diameter by $0.90 \mathrm{in.}$ long. 


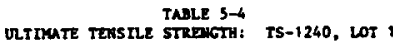

\begin{tabular}{|c|c|c|c|c|c|c|c|c|c|c|c|c|c|}
\hline \multirow{3}{*}{$\begin{array}{l}\text { CA } \\
\text { Log } \\
\text { Ho. }\end{array}$} & \multirow{3}{*}{$\begin{array}{l}\text { vec } \\
\text { Log } \\
\text { so. }\end{array}$} & \multicolumn{12}{|c|}{ Kean Strength, pa1 (Standard Devitat ton, psi) (No. of Replicatee) } \\
\hline & & \multicolumn{6}{|c|}{ Axles Oxtentetion } & \multicolumn{6}{|c|}{ Rediel Or lentation } \\
\hline & & Nac & $\operatorname{kgB}$ & QLC & QLI & $\mathrm{Ec}$ & $\mathbf{m z}$ & sac & sus & QRE & Q212 & xc & $\mathbf{E E}$ \\
\hline $5651-72$ & 32 & & & $1792(136) \times[18]$ & $1851(287)[21]$ & $1693(260)(19)$ & $1959(137)[19]$ & & & $2035(105)[13]$ & $2053(151)[16]$ & $1775(519)[17]$ & $2087(110)[17]$ \\
\hline $5651-73$ & 40 & $1796(117)$ [6] & $2290(357) \quad[5]$ & & & & & 1436 (159) $[6]$ & $1837(109)[6]$ & & & & \\
\hline $5651-73^{(a)}$ & 40 & $1571(252)\{201$ & $2315(440)[20]$ & & & & & 1383 (298) $[201$ & $1991 \quad(285)[20]$ & & & & \\
\hline $565 i-74$ & 42 & 2007 (131) [201 & $2226(236)[19]$ & & & $2211(187)\{20\}$ & $2271(193)[20\}$ & $1716(305)[17]$ & $1744(307)[18]$ & & & $2186(380)[15]$ & $2090(345)[19]$ \\
\hline $5651-75$ & 46 & $2696(177)[17]$ & $2741(233)[19]$ & & & $1886(278)[20]$ & $2096(238)[19]$ & $1363(683)[17]$ & $1912(471)[16]$ & & & 2014 (68B) $[16]$ & 2049 (223) [1] \\
\hline $6484-29$ & 8-15- & $2304(179)[19]$ & $2511(275)[16]$ & & & $2365 \quad(198)\{20\}$ & $2437(260)[17]$ & 1301 (192) [19] & 1390 (293) [19] & & & $1954(436)[19]$ & $2127(306)[20]$ \\
\hline Mean & & $2097(462)[82]$ & $2446 .(368)[79]$ & $1792(136)[18]$ & $\mid 1851(287) \quad 1211$ & $1990(415) \quad[79\}$ & 2185 (272) [75] & $1430(413)[79]$ & 1762 (395) $[79]$ & $20.035(105)[13]$ & 2053 (151) $[16]$ & $1975(526)[67]$ & $2090(263)[73\}$ \\
\hline
\end{tabular}


The mean tensile strength of $\mathrm{H}-451$, lot 426, ranged from $1980 \pm 234$ psi at MLC to $2758 \pm 269$ psi at MLE in the axial direction and from 1560 $\pm 304 \mathrm{psi}$ at $\mathrm{MLC}$ to $2208 \pm 326 \mathrm{psi}$ at $\mathrm{EE}$ in the radial direction. The mean tensile strength of TS-1240, lot 1, ranged from $2097 \pm 442$ psi at MLC to $2446 \pm 368 \mathrm{psi}$ at MLE in the axial direction and from $1430 \pm 413$ psi at MLC to $2090 \pm 263$ psi at EE in the radial direction.

The mean $\log$ strengths for $\mathrm{H}-451$ graphite, lot 426, calculated in the same manner as the mean $\log$ density (Section 5.2.1), were 2538 psi for the axial direction and 2032 psi for the radial direction. The corresponding values for TS-1240, 1ot 1, were 2247 psi for the axial direction and 1970 psi for the radial direction.

H-451 logs manufactured with a blend of cokes A and B, lots 408 and 426, had significantly higher tensile strengths at all $10 g$ positions than those manufactured with coke A, lot 266 (see Tab1e 5-3).

Standard deviations for H-451 ranged from approximately 250 to 350 psi and those for TS-1240 ranged from 300 to 400 psi. The radial strength values of TS-1240, $\log 5651-75$, had standard deviations of about 680 psi at the MLC and EC positions. These were the highest deviations observed.

Large log-to-log variations in mean strength were observed for a given position in both graphites. A maximum variation of $1125 \mathrm{psi}$ in the mean strengths of the logs of TS-1240 was observed at the MLC position in the axial direction. The maximum variation for $\mathrm{H}-451$, lot 426, was $955 \mathrm{psi}$ at EC in the axial direction. No systematic variations in strength were observed from position to position within a $10 \mathrm{~g}$ for either graphite grade.

\subsubsection{Modulus of Elasticity}

The modulus of elasticity data are summarized in Tables 5-5 and 5-6. The modulus of elasticity of $\mathrm{H}-451$ and $\mathrm{TS}-1240$ varied with position and orientation in a $\log$ in the same manner as tensile strength. 
TABLE 5-5

MODULUS OF ELASTICITY: $\mathrm{H}-451$

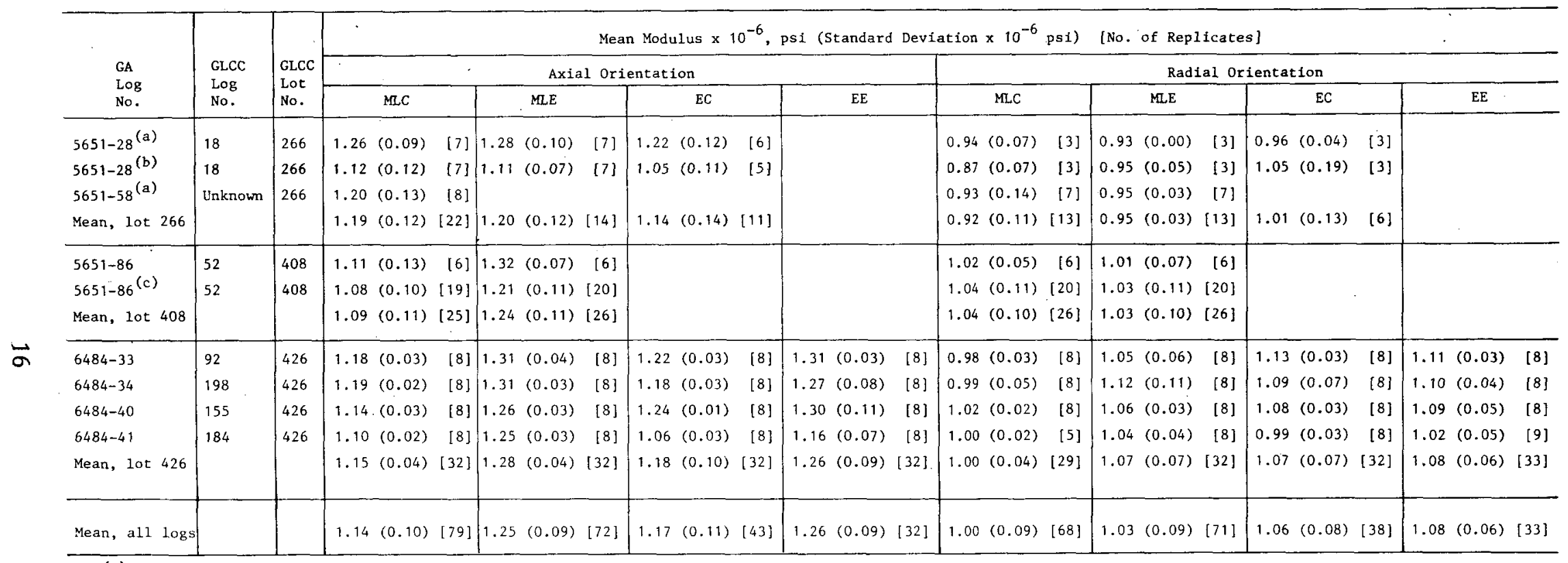

(a) Modulus of elasticity taken as chord modulus between 250 and $500 \mathrm{psi}$ on 0.505 -in.-diameter by 4.0-in.-long specimens but tested to fallure without cycling.

(b) Same as (a) except specimens were $0.25 \mathrm{in}$. in diameter by $0.90 \mathrm{in}$. long.

(c) Specimens were $0.25 \mathrm{in}$. in diameter by $0.90 \mathrm{in}$. long. 


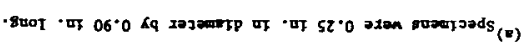

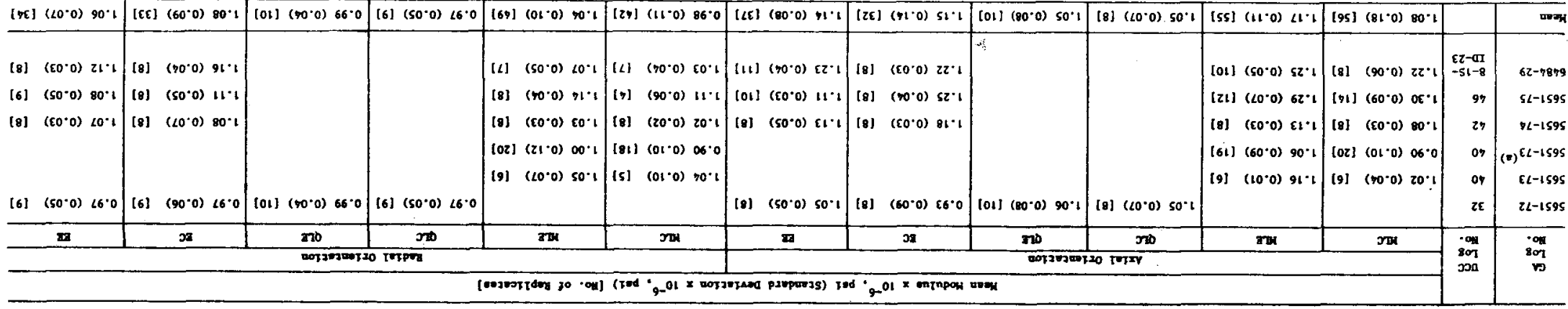

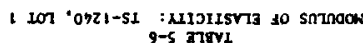


The mean modulus of $\mathrm{H}-451$, lot 426 , ranged from $1.15 \times 10^{6}$ psi at MLC to $1.28 \times 10^{6}$ psi at MLE in the axial direction and from $1.00 \times 10^{6}$ psi at MLC to $1.08 \times 10^{6}$ psi at EE in the radial direction. The mean modulus of TS-1240, lot 1 , ranged from $1.08 \times 10^{6} \mathrm{psi}$ at MLC to $1.17 \times 10^{6}$ psi at MLE in the axial direction and from $0.98 \times 10^{6}$ psi at MLC to $1.08 \times$ $10^{6}$ psi at EC in the radial direction.

The standard deviations of the moduli for $\mathrm{H}-451$, lot 426, ranged from $0.04 \times 10^{6}$ psi to $0.10 \times 10^{6} \mathrm{psi}$ and those for $\mathrm{TS}-1240$, 1ot 1 , ranged from $0.07 \times 10^{6}$ psi to $0.18 \times 10^{6}$ psi.

The mean log moduli for H-451 graphite, lot 426, calculated in the same manner as the mean $10 \mathrm{~g}$ density (Section 5.2.1) were $1.24 \times 10^{6} \mathrm{psi}$ for the axial direction and $1.07 \times 10^{6}$ psi for the radial direction. The corresponding values for TS-1240, lot 1 , were $1.15 \times 10^{6}$ psi for the axial direction and $1.05 \times 10^{6}$ psi for the radial direction.

Significant $\log$-to-log variations in mean modulus were observed for a given position for both graphites. For TS-1240, variations observed in the axial direction ranged from $0.18 \times 10^{6} \mathrm{psi}$ at EE to $0.40 \times 10^{6} \mathrm{psi}$ at MLC and in the radial direction from $0.11 \times 10^{6}$ psi at EE to $0.21 \times 10^{6}$ psi at MLC. For H-451, 1ot 426, the variations ranged from $0.06 \times 10^{6}$ psi at MLE to $0.18 \times 10^{6} \mathrm{psi}$ at EC in the axial direction and from $0.04 \times 10^{6} \mathrm{psi}$ at MLC to $0.14 \times 10^{6} \mathrm{psi}$ at $\mathrm{EC}$ in the radial direction. The variations in modulus of both graphites at a given position were largest in the axial direction.

\subsubsection{Strain at Fracture}

The data for strain at fracture are given in Appendix B in Tables B-1 through B-17.

The strain at fracture for $\mathrm{H}-451$ and $\mathrm{TS}-1240$ was in the range 0.25 to $0.35 \%$. The strain at fracture was lowest for both axial and radial specimens at the MLC of all logs and increased from the center to the edges. 
For TS-1240 the strain at fracture was generally greater in the axial direction than in the radial direction at MLC and MLE, whereas it was greater in the radial direction at $\mathrm{EC}$ and $\mathrm{EE}$. A systematic variation in the strain at fracture from log-to-log or lot-to-lot was not observed in $\mathrm{H}-451$.

\subsubsection{Poisson's Ratio}

The Poisson's ratio data are summarized in Table 5-7. The Poisson's ratio values, $\bar{v}_{i j}$, are identified in standard tensor notation (Ref. 5) according to the orthogonal axes within a $\log$ as shown in Fig. 5-2.

Poisson's ratio was measured on $\mathrm{H}-451$ and $\mathrm{TS}-1240$ in the axial and radial directions at several locations in a $\log$. Poisson's ratio decreased with increasing stress during initial loading and reached a constant value, independent of stress, after two or three loadings.

When stresses were applied in a radial direction $\left(\sigma_{1}\right.$ or $\left.\sigma_{2}\right)$ to $\mathrm{H}-451$ MLC specimens, $\bar{v}_{13}=\bar{v}_{23}=0.110 \pm 0.009$ and $\bar{v}_{12}=\bar{v}_{21}=0.108 \pm 0.008$. When stresses were applied in the axial direction $\left(\sigma_{3}\right)$ to H-451 MLC specimens, $\bar{v}_{31}=\bar{v}_{32}=0.127 \pm 0.013$. When stresses were applied in the axial direction $\left(\sigma_{3}\right)$ to H-451 MLE specimens, $\bar{v}_{32}=0.121 \pm 0.005$ and $\bar{v}_{31}=0.114$ \pm 0.006 . When stresses were applied in the axial direction $\left(\sigma_{3}\right)$ to $\mathrm{H}-451$ EE specimens, $\bar{v}_{32}=0.110 \pm 0.010$ and $\bar{v}_{31}=0.117 \pm 0.006$.

When stresses were applied in the radial direction $\left(\sigma_{1}\right.$ or $\left.\sigma_{2}\right)$ to TS-1240 QLC specimens, $\bar{v}_{31}=\bar{v}_{32}=0.129 \pm 0.007$. When stresses were applied in the axial direction $\left(\sigma_{3}\right)$ to TS-1240 QLC specimens, a Poisson's ratio (unknown orientation of transverse strain) of $0.129 \pm 0.007$ was obtained.

\subsubsection{Therma1 Expansivity}

Thermal expansivity data are summarized in Tables 5-8 and 5-9. The mean thermal expansivity of $\mathrm{H}-451$, lot 426 , ranged from $3.89 \times 10^{-6}{ }^{\circ} \mathrm{C}^{-1}$ to 
TABLE $5-7$
POISSON'S RATTO: H-451 AND TS-1240

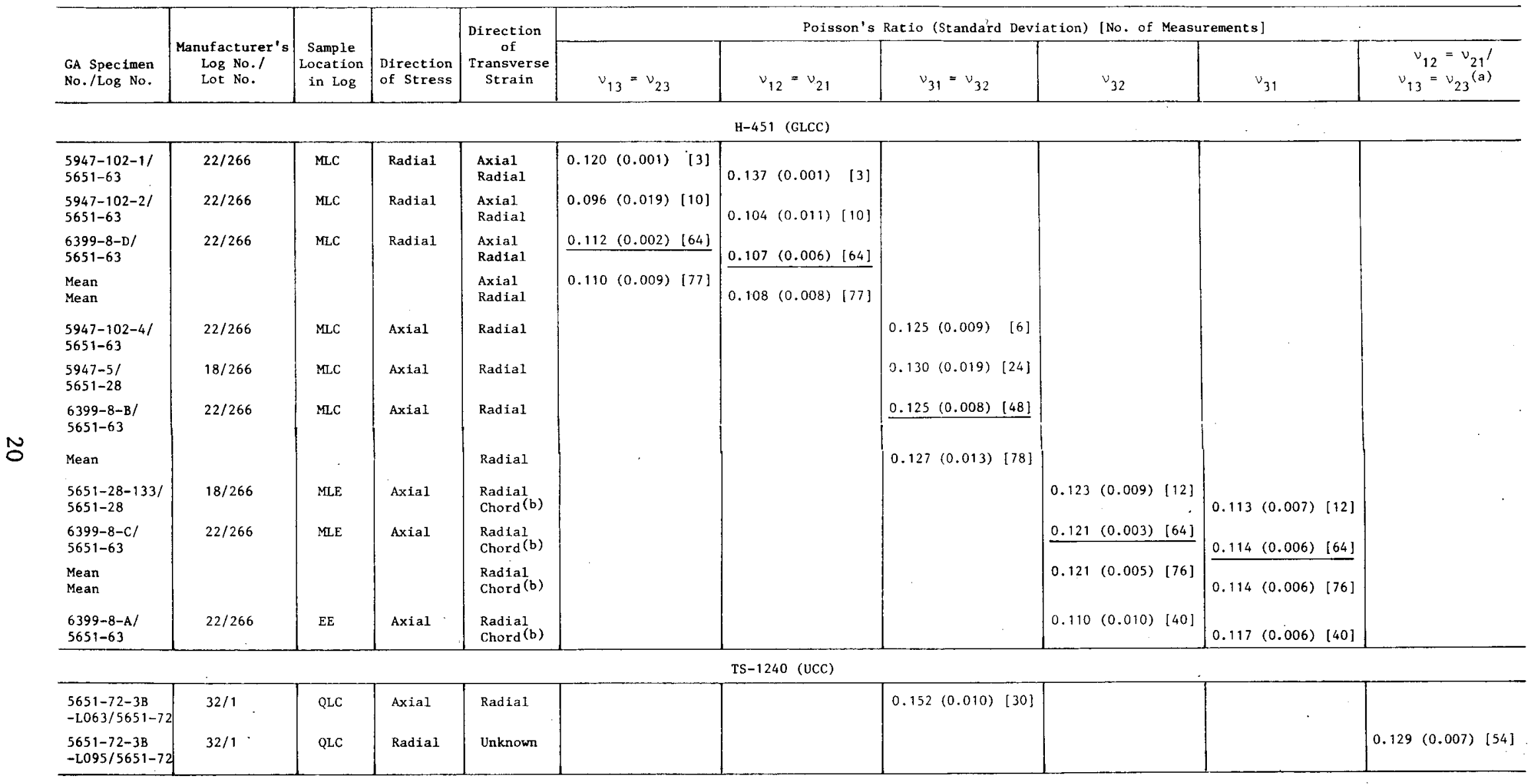

(a) Orientation of sample in $\log$ with respect to directions of measured transverse strains was unknown. Poisson's ratio represents "mean" of various components indicated.

(b) Perpendicular to radius in radial plane. 


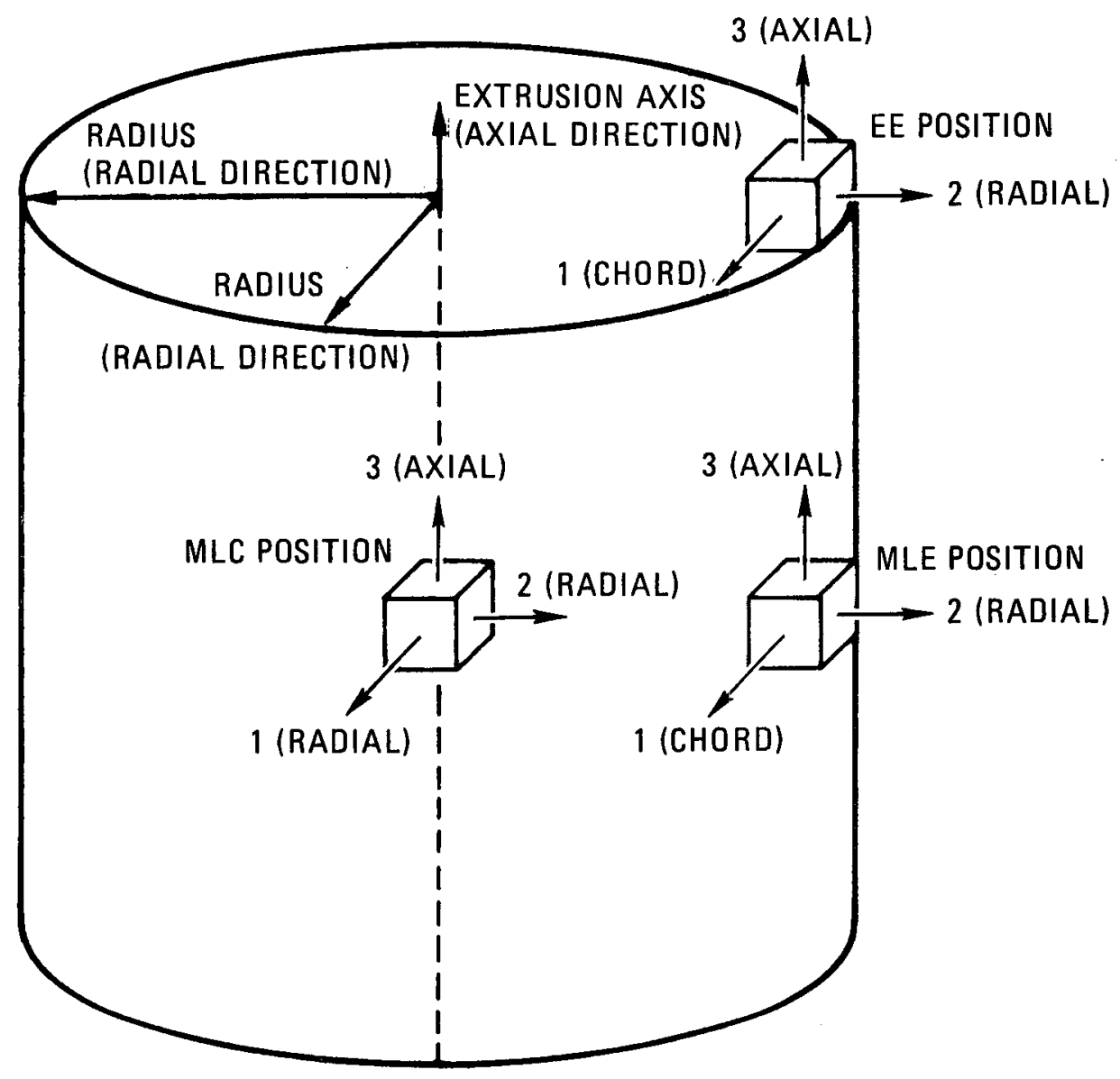

Fig. 5-2. Orientation of orthogonal axes for definition of Poisson's ratio 
TABLE 5-8
THERMAL EXPANS IVITY: $\quad$ H-451

\begin{tabular}{|c|c|c|c|c|c|c|c|c|c|c|c|c|c|c|c|c|c|c|}
\hline \multirow{3}{*}{$\begin{array}{l}\text { GA } \\
\text { Log } \\
\text { No. }\end{array}$} & \multirow{3}{*}{\begin{tabular}{|l|} 
GLCC \\
Log \\
No.
\end{tabular}} & \multirow{3}{*}{$\begin{array}{l}\text { GLCC } \\
\text { Lot } \\
\text { No. }\end{array}$} & \multicolumn{16}{|c|}{ Mean Thernal Expansivity $\times 10^{6}{ }^{\circ} \mathrm{C}^{-1}$ (Standard Deviation $\times 10^{6}{ }^{\circ} \mathrm{C}^{-1}$ ) [No. of Replicates] } \\
\hline & & & \multicolumn{6}{|c|}{ Axial } & \multicolumn{10}{|c|}{ Radial } \\
\hline & & & & MLC & MLE & EC & $\mathrm{EE}$ & & & $\mathrm{MLC}$ & & MLE & & & EC & & EE & \\
\hline $5651-28$ & 18 & 266 & 3.45 & $(0.03) \quad[5]$ & $3.45(0.06) \quad[6]$ & & & & 4.41 & $(0.13) \quad[6]$ & 4.49 & $9(0.16)$ & {$[6]$} & & & & & \\
\hline $5651-86$ & 52 & 408 & 4.18 & $(0.15) \quad[8]$ & $4.19(0.11)[10]$ & & & & 4.63 & $(0.17) \quad[10]$ & 4.57 & $7(0.21)$ & {$[12]$} & & & & & \\
\hline $6484-33$ & 92 & 426 & 4.08 & $(0.14) \quad[8]$ & $3.86(0.20) \quad[8]$ & $4.00(0.18) \quad[8]$ & $3.88(0.09)$ & [8] & 4.51 & $(0.29) \quad[8]$ & 4.65 & $5(0.13)$ & {$[8]$} & 4.70 & $(0.14) \quad[8]$ & 4.70 & $(0.21)$ & [8] \\
\hline $6484-34$ & 198 & 426 & 4.10 & $(0.10) \quad[8]$ & $3.92(0.19) \quad[8]$ & $\left.\begin{array}{lll}4.31 & (0.08) & 18\end{array}\right]$ & $4.13(0.12)$ & [8] & 4.63 & $(0.20) \quad[8]$ & 4.66 & $6(0.25)$ & [8] & 4.55 & $5(0.27) \quad[8]$ & 4.59 & $9(0.10)$ & [8] \\
\hline $6484-41$ & 184 & 426 & 3.92 & $(0.07) \quad[8]$ & $3.90(0.09) \quad[8]$ & $3.90(0.13) \quad[8]$ & $3.89(0.09)$ & {$[8]$} & 4.42 & $(0.18) \quad[8]$ & 4.50 & $(0.18)$ & {$[8]$} & 4.43 & $3(0.16) \quad[8]$ & 4.59 & 9. $(0.18)$ & {$[8]$} \\
\hline Mean, lot 426 & & & 4.03 & $(0.13)[24]$ & $3.89(0.16) \quad[24]$ & $4.07 \quad(0.22) \quad[24]$ & $3.97(0.15)$ & [24] & 4.52 & $(0.23) \quad[24]$ & 4.60 & $(0.20)$ & {$[24]$} & 4.56 & $6(0.22) \quad[24]$ & 4.63 & $3(0.17)$ & [24] \\
\hline Mean, all $\operatorname{logs}$ & & & 3.98 & $(0.25)[37]$ & $3.90(0.27) \cdot[40]$ & $4.07(0.22) \quad[24]$ & $3.97(0.15)$ & [24] & 4.53 & $(0.21)[40]$ & 4.58 & $8(0.20)$ & [42] & 4.56 & $6(0.22) \quad[24]$ & 4.63 & $3(0.17)$ & {$[24]$} \\
\hline
\end{tabular}


TASLE S-9
THERMT EOPANSTVIT:

\begin{tabular}{|c|c|c|c|c|c|c|c|c|c|c|c|c|c|}
\hline \multirow{3}{*}{$\begin{array}{l}\text { GA } \\
\text { Log } \\
\text { So. }\end{array}$} & \multirow{3}{*}{\begin{tabular}{|l|} 
vcc \\
Log \\
No.
\end{tabular}} & \multicolumn{12}{|c|}{ Mean Thermal Expansivity $\times 10^{6} \cdot \mathrm{c}^{-1}$ (Standard Deviaction $\times 10^{6}{ }^{\circ} \mathrm{c}^{-1}$ ) (No. of Rep11cates) } \\
\hline & & \multicolumn{6}{|c|}{ Axial } & \multicolumn{6}{|c|}{ 2ed181 } \\
\hline & & $\overline{\text { KaC }}$ & RLE & Q2C & Que & $\bar{x}$ & BE & nec & Ma: & Q2E & QLE & Ec & $\mathbf{z x}$ \\
\hline $\begin{array}{l}5651-72 \\
5651-73\end{array}$ & 32 & $4.26(0.22)$ [B] & $4.28(0.25)[11]$ & $4.05(0.21)[8]$ & $4.12(0.16)[8]$ & $0.48(0.10)$ & $4.49 \quad(0.32) \quad[81$ & $4.84(0.09) \quad[8]$ & $4.73(0.21)[12]$ & $6.50(0.11)(8)$ & $4.68(0.20)(8)$ & $4.84(0.24) \quad[8]$ & $4.96(0.36)-[8]$ \\
\hline $5651-74$ & 42 & $4.33 \quad(0.06) \quad[8]$ & $4.26(0.07) \quad[B]$ & & & $4.29(0.16) \quad[8]$ & $4.28(0.19) \quad[8]$ & $4.57(0.18)$ (8) & $4.62(0.12) \quad\{8\}$ & & & $4.63(0.11) \quad(8)$ & $4.84(0.11)$ [8] \\
\hline $5651-75$ & 46 & $3.90(0.09) \quad[8]$ & $4.18(0.13) \quad[8]$ & & & $3.69(0.13) \quad[8\}$ & $\begin{array}{lll}4.44 & (0.35) & {[81}\end{array}$ & $4.36(0.16) \quad(8)$ & $4.70(0.11) \quad[8]$ & & & $\mid 4.27 \quad(0.19) \quad[8]$ & $4.95(0.17) \quad$ (8) \\
\hline $6484-29$ & $\left|\begin{array}{c}8-15- \\
1 \mathrm{p}-23\end{array}\right|$ & $4.63(0.17) \quad[8]$ & $4.59(0.16) \quad[8]$ & & & $\begin{array}{lll}4.61 & (0.21) & {[8]}\end{array}$ & $4.69(0.15) \quad[8]$ & $5.00(0.12) \quad[8]$ & $5.18(0.20)[8]$ & & & $5.39(0.30) \quad[8]$ & $\left.\begin{array}{lll}5.03 & (0.12) & {[8]}\end{array}\right]$ \\
\hline a & & $4.28(0.30)[32]$ & $4.32(0.22)[35]$ & $\begin{array}{|lll|}4.05 & (0.21) & {[8]} \\
\end{array}$ & $\begin{array}{lll}4.12 & (0.16)[8]\end{array}$ & $\begin{array}{llll}4.27 & (0.39) & {[321} \\
\end{array}$ & $4.48(0.25)[32]$ & $4.69(0.28)[32\}$ & $4.80(0.27)[36]$ & $4.50(0.11)[8]$ & $\begin{array}{lll}4.68 & (0.20) & {[8]}\end{array}$ & $4.78(0.46)[32]$ & $4.95(0.22)[32]$ \\
\hline
\end{tabular}


$4.07 \times 10^{-6}{ }^{\circ} \mathrm{C}^{-1}$ in the axial direction and $4.52 \times 10^{-6}{ }^{\circ} \mathrm{C}^{-1}$ to $4.63 \mathrm{x}$ $10^{-6}{ }^{\circ} \mathrm{C}^{-1}$ in the radial direction. The mean thermal expansivity of TS-1240, lot 1 , ranged from $4.05 \times 10^{-6}{ }^{\circ} \mathrm{C}^{-1}$ to $4.48 \times 10^{-6}{ }^{\circ} \mathrm{C}^{-1}$ in the axial direction and $4.5 \times 10^{-6}{ }^{\circ} \mathrm{C}^{-1}$ to $4.95 \times 10^{-6}{ }^{\circ} \mathrm{C}^{-1}$ in the radial direction. There was no significant variation of thermal expansivity as a function of specimen location in a $\log$ for either material.

\subsubsection{Thermal Conductivity}

The thermal conductivity data are summarized in Tables 5-10 and 5-11. The mean thermal conductivity of $\mathrm{H}-451$, lot 426 , was $0.155 \pm 0.009 \mathrm{cal} / \mathrm{cm}-$ sec- ${ }^{\circ} \mathrm{C}$ at $800^{\circ} \mathrm{C}$ in the axial direction and $0.150 \pm 0.010 \mathrm{ca} 1 / \mathrm{cm}-\mathrm{sec}-{ }^{\circ} \mathrm{C}$ in the radial direction. The mean thermal conductivity of TS-1240 was $0.148 \pm$ $0.014 \mathrm{cal} / \mathrm{cm}-\mathrm{sec}-{ }^{\circ} \mathrm{C}$ at $800^{\circ} \mathrm{C}$ in the axial direction and $0.147 \pm 0.012 \mathrm{cal} /$ $\mathrm{cm}-\mathrm{sec}-{ }^{\circ} \mathrm{C}$ in the radial direction.

\subsubsection{Impurity Content}

\subsubsection{Neutronic Absorbtion Impurities}

Two types of neutron absorbing impurity elements, burnable and nonburnable, are of importance to the neutronic design and operation of an LHTGR. The impurity data are summarized in Table B-37. The upper bound nonburnable boron equivalent value was $0.9486 \mathrm{ppm}$ for $\mathrm{H}-451$ and $0.7839 \mathrm{ppm}$ for TS -1240 . The upper bound burnable boron equivalent value was 3.2922 ppm for $\mathrm{H}-451$ and $1.8560 \mathrm{ppm}$ for TS-1240. In most cases, the upper bound boron equivalent values reflect the lower limits of detection of the analytical methods used to detect the various elements.

\subsubsection{Ash and Other Important Impurities}

The contents of ash and other important impurities are summarized in Table 5-12. 
TABLE 5-10

THERMAL CONDUCTIVITY: $\mathrm{H}-451$

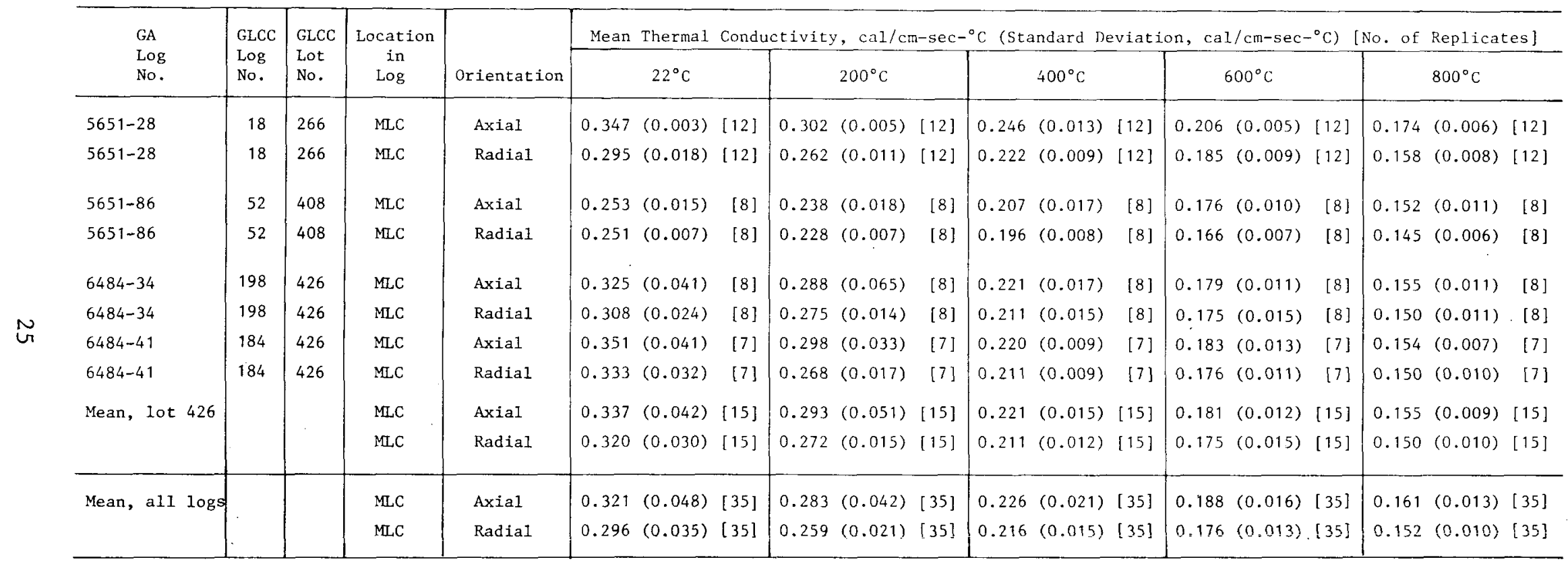


TABLE 5-11

THERMAL CONDUCTIVITY: TS-1240, LOT 1

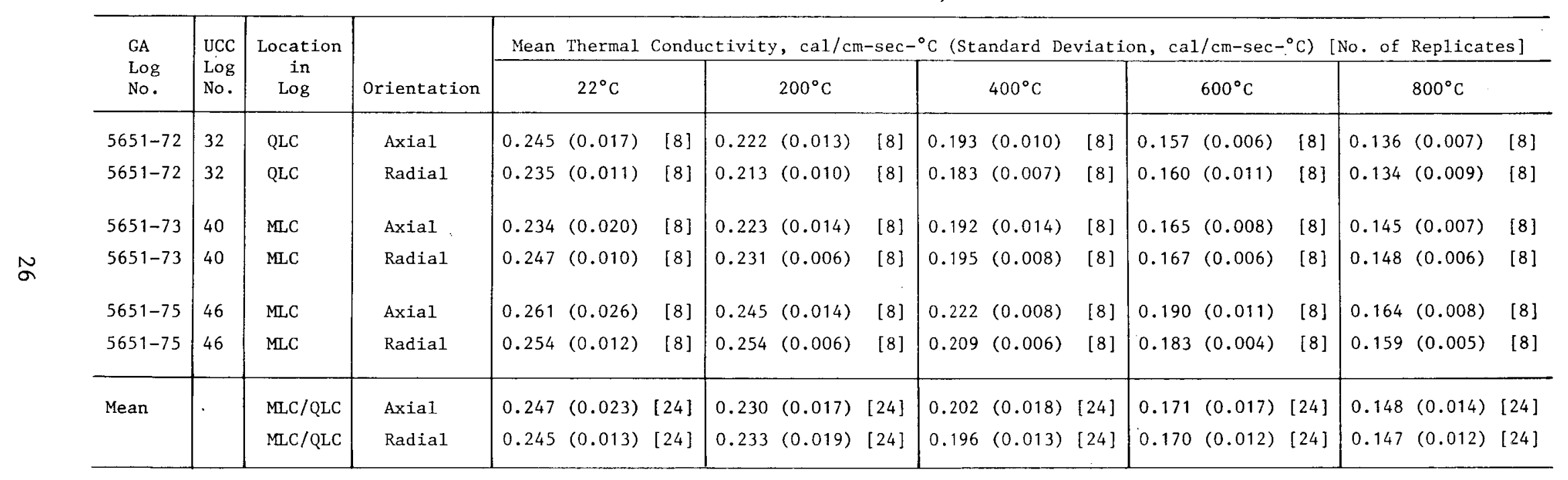


TABLE 5-12

IMPURITY CONTENT

[in $\operatorname{ppm}^{(\mathrm{a})}$ ]

\begin{tabular}{l|l|l|l|l|l|l|l|l|l|l|l|l|l|l}
\hline $\begin{array}{l}\text { Graphite } \\
\text { H-451 } \\
\text { (1ot 426) }\end{array}$ & 45 & 2.5 & 3.2 & 0.5 & 1.3 & 1.5 & 4.3 & 21.9 & 21.3 & 10.3 & 0.9 & 7.1 & -- & -- \\
TS-1240 & 78.7 & 0.7 & 11.1 & 18.9 & 16.0 & 9.4 & 4.1 & 22.5 & 14.9 & 10.4 & 1.0 & 6.0 & 2.9 & 4.6 \\
\hline
\end{tabular}

(a) Less than value given. 


\section{DISCUSSION AND CONCLUSIONS}

\subsection{DISCUSSION}

\subsection{1. $\underline{\mathrm{H}-451}$}

The initial lots of grade H-451 were manufactured with a blend of petroleum-based near-isotropic calcined cokes from two sources. The objective of the development program by GLCC was to optimize the properties and irradiation behavior of H-451 graphite for LHTGR design by choosing a blend of two calcined petroleum cokes for the filler. Great Lakes Carbon Co. foresees uncertainties in the procurement of petroleum coke over the long term with changing feedstocks and changing sources of coke. Therefore, the objective of their internal program is to establish coke technology that wil1 permit them to purchase coke for future $\mathrm{H}-451$ production from different sources that will reproduce the structure and properties of the prototype material currently under evaluation. Lot 426, produced from a blend of cokes $A$ and $B$, has been selected as the prototype lot for future production of commercial $\mathrm{H}-451$.

The mean property values of prototype lot 426 are given in Section 5.2. These data, except density, characterize grade H-451 which will be manufactured for Fort St. Vrain reload fuel and replaceable reflector elements. Commercial H-451 will be manufactured to a mean lot density value of $1.74 \mathrm{~g} / \mathrm{cm}^{3}$ and a minimum $\log$ value of $1.69 \mathrm{~g} / \mathrm{cm}^{3}$.

Lot 440, manufactured with a blend of cokes $B$ and $C$, is currently under evaluation. Lot 440 was manufactured with a mean density of approximately $1.77 \pm 0.01 \mathrm{~g} / \mathrm{cm}^{3}$. Comparisons of property and irradiation data will be made between lots 426 and 440 to assess the effect of the different 
coke blends and densities. Several logs from the manufacturing run for Fort St. Vrain reload segment 9 will be evaluated to assess how well the commercial product matches the prototype lots 426 and 440 .

\subsection{2. $\mathrm{TS}-1240$}

The initial lot of TS-1240 was manufactured with a single-source near-isotropic calcined petroleum coke. The objective of the development program at UCC was to produce a full-size prototype grade for evaluation. The mean property values of lot 1 are given in Section 5.2. These data are representative of grade TS-1240 in its present stage of development. One additional experimental lot of TS-1240 is being manufactured. The objectives of this further development are: (1) to reproduce the properties and irradiation behavior of the first lot, and (2) to improve the product.

\subsection{CONCLUSIONS}

Grade H-451 has been developed to the stage where a commercial order has been placed for use as reload elements in segment 9 of the Fort St. Vrain reactor. The demonstrated superior irradiation behavior and properties of H-451 over the original Fort St. Vrain needle-coke graphite (H-327) should improve the fuel element performance. 
The authors wish to express their thanks to L. Bailey, C. H. Richards, Jr., J. R. Whaley, M. G. Dunlap, F. Hogan, H. H. Evans, and D. W. Stevens for performing the laboratory work.

L. H. Juel and R. F. Peters of Great Lakes Carbon Company and A. E. Goldman of Union Carbide Corporation are acknowledged with thanks for their cooperation in furnishing materials and their helpful comments during the course of work. 


\section{REFERENCES}

1. Engle, G. B., et a1., "Development Status of Near-Isotropic Graphites for Large HTGRs," USAEC Report GA-A12944, General Atomic Company, June 1, 1974.

2. Price, R. J., and L. A. Beavan, "Final Report on Graphite Irradiation Test OG-1," USAEC Report GA-A13089, General Atomic: Company, August 1, 1974.

3. Price, R. J., and L. A. Beavan, "Final Report on Graphite Irradiation Test OG-2," ERDA Report GA-A13556, Genera1 Atomic Company, December 15, 1975.

4. Cobb, H. R. W., G. B. Engle, and S. J. S. Parry, "Characterization of Production-Grade H-327 Graphite for HTGR Design," in Proceedings of Conference on Continuum Aspects of Graphite Design., Gatlinburg, Tennessee, November 9-12, 1970, USAEC Report CONF-701105, p. 98.

5. Nye, J. F., Physical Properties of Crystals, Oxford University Press, London, 1967, pp. 1-8. 
APPENDIX A

EXPERIMENTAL METHODS

A. 1. DENSITY

Bulk densities were measured according to ASTM Standard Test C-559.

\section{A.2. TENSTLE PROPERTIES}

\section{A.2.1. Stress-Strain Curves}

Tensile stress-strain curves were obtained in air at room temperature on unirradiated samples using an Instron tensile machine. Tensile tests were conducted on standard (0.505-in. diameter by 3 to 4 in. long) and subsize (0.250-in. diameter by 0.9 in. long) samples using a crosshead speed of 0.005 to $0.01 \mathrm{in./min.} \mathrm{Strains} \mathrm{were} \mathrm{measured} \mathrm{for} \mathrm{subsize} \mathrm{samples}$ using a 0.5-in. gage length nonaveraging clip-on extensometer. A 2.0-in. gage length averaging extensometer was used on the standard samples. The samples were fixed to metal end pieces with high-strength epoxy cement, and the load was applied through roller-link chains to maintain uniaxial alignment during testing.

All samples were tested using a cyclic loading method. Each sample was loaded to $1000 \mathrm{psi}$ and the crosshead reversed. After unloading to 100 psi, the crosshead was again reversed, and the sample was loaded until fracture occurred. A typical stress-strain curve obtained by this method is shown in Fig. A-1. 


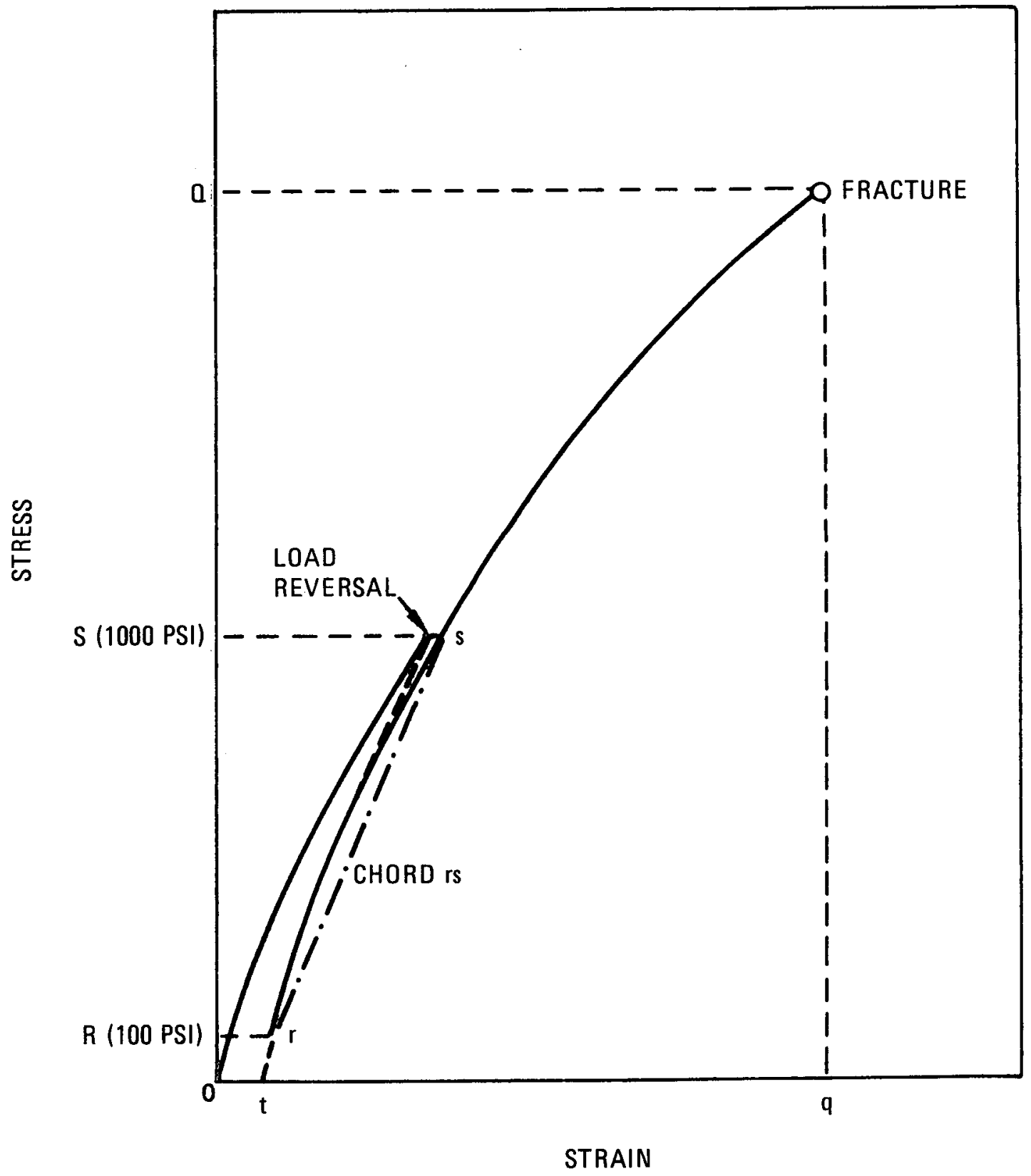

Fig. A-1. Cyclic stress-strain curve for graphite 
Because of the inelastic component in the deformation of graphite, the unloading curve does not retrace the initial loading curve but reaches zero load with a positive "permanent set." The second loading curve generally superimposes on the unloading curve, and beyond the first strain reversal point it forms a continuation of the initial loading curve.

\section{A.2.2. Tensile Strength}

Samples tested for ultimate tensile strength only (no stress-strain curve) were loaded to failure without crosshead reversal. The tensile strength of all samples was calculated by dividing the load at fracture (stress corresponding to point $Q$ on stress-strain curve, Fig. A-1) by the original cross-sectional area of the specimen.

\section{A.2.3. Modulus of Elasticity}

The modulus of elasticity of each specimen was calculated from its stress-strain curve as the slope of the chord (chord modulus) drawn between points on the second loading portion of the curve corresponding to stresses of 100 and 1000 psi (chord rs, Fig. A-1). This chord modulus represents the effective modulus of a graphite component after its first stress excursion.

\section{A.2.4. Strain at Fracture}

The strain at fracture for all samples was taken as the maximum strain as indicated on the stress-strain curve (strain corresponding to point $q$, Fig. $A-1$ ).

\section{A.2.5. Permanent Set}

The permanent set (residual strain) remaining in the sample after load reversal was obtained from the stress-strain curve as the value of strain extrapolated from the load reversal curve to zero stress (strain corresponding to point $t$, Fig. A-1). 


\section{A.2.6. Poisson's Ratio}

Poisson's ratio was obtained on samples $0.505-i n$, in diameter by 5 in. long and 0.5 in. by 0.5 in. by 5 in. long using a strain gage method. Multiple (two to four) biaxial strain gages, equidistant around the cross section, were aligned with the longitudinal and transverse directions of each specimen and attached in position with an epoxy cement. The samples were fixed to end pieces with a high-strength epoxy cement and were loaded in tension in steps to stresses as high as 1200 psi while recording the strains from each gage. The load was applied through roller-link chains to maintain uniaxial alignment during testing. The transverse and longitudinal strains for each gage were measured at each load using a digital strain indicator. Poisson's ratio was calculated from the ratio of transverse and longitudinal strains for each biaxial gage after first conrecting each strain for transverse sensitivity.

\section{A.3. THERMAL CONDUCTIVITY}

Thermal diffusivity measurements were made on disc samples, 0,45 in, in diameter by 0.050 in. thick, by the heat pulse method (ASTM Standard Test Method $\mathrm{C}-741)$. The thermal conductivity was calculated from the following equation:

$$
k=\alpha C_{p} \rho,
$$

where $\alpha=$ thermal diffusivity

$$
\begin{aligned}
& C_{p}=\text { heat capacity } \\
& \rho=\text { density }
\end{aligned}
$$

Specimens were measured at $100^{\circ} \mathrm{C}$ intervals from room temperature to $800^{\circ} \mathrm{C}$. 


\section{A.4. THERMAL EXPANSIVITY}

Thermal expansivity was measured according to ASTM Standard Test Method E-228 except measurements were made on cylindrical samples, 0.25 in. in diameter by 0.9 in. long, using a silica dilatometer. Measurements were made between room temperature and $1000^{\circ} \mathrm{C}$.

\section{A.5. CHEMICAL IMPURITIES}

The graphites were analyzed for ash for all major burnable* and nonburnable** impurities except oxygen and nitrogen. The analytical methods used are given below.

\section{A.5.1. $\underline{\text { Ash }}$}

Analyses for ash were conducted by burning 10-g samples to constant weight in a covered crucible in still air (waffle furnace) at $900^{\circ}$ to $1000^{\circ} \mathrm{C}$.

\section{A.5.2. Standard Emmission Spectroscopy}

Procedures for analysis by emission spectroscopy on unconcentrated and preconcentrated (ashed) samples are described in Ref. A-1.

Determinations of the effects of preconcentration (by ashing) on impurity levels in graphite were made by analyzing ashed, doped standards. Samples were prepared by doping a high-purity spectrographic carbon with

* Burnable impurities are those elements whose cross-section and isotopic distribution are such that their neutronic poisoning effects are virtually eliminated by neutron absorption during the cycle. The major burnable impurities are $\mathrm{B}, \mathrm{Cd}, \mathrm{Eu}, \mathrm{Gd}, \mathrm{Sm}$, and $\mathrm{Li}$.

** Nonburnable impurities are those impurities which retain a considerable fraction of their poisoning effect after a full-power year of operation. The significant nonburnable impurities are $\mathrm{Ag}, \mathrm{A} 1, \mathrm{Ba}, \mathrm{Ca}, \mathrm{Cl}, \mathrm{Co}, \mathrm{Cr}, \mathrm{Cs}, \mathrm{Cu}$, Dy, Er, Fe, Hf, Ho, In, K, La, Sn, Lu, Mg, Mo, Mn, Na, Nd, Ni, Pr, S, Sb, Sc, $\mathrm{Si}, \mathrm{Ta}, \mathrm{Tb}, \mathrm{Ti}, \mathrm{Tm}, \mathrm{V}, \mathrm{W}, \mathrm{Yb}, \mathrm{P}, \mathrm{Pb}, \mathrm{Rb}, \mathrm{T} 1, \mathrm{Zn}, \mathrm{Zr}, \mathrm{Bi}, \mathrm{Nb}, \mathrm{N}, \mathrm{O}, \mathrm{Sr}, \mathrm{Ce}$, and $\mathrm{Be}$. 
$1 \mathrm{ppm}$ each of all primary nonburnable and burnable elements in the form of salts. Procedures for ashing and emission spectrographic analysis are described in Ref. A-1.

Results obtained were used to determine losses that occurred from ashing. These results are shown in Table A-1.

\section{A.5.3. Spark Source Mass Spectrometry (Accu-Labs Research, Inc.)}

Semiquantitative analyses by spark source mass spectrometry (Geomascan Analysis) were conducted by Accu-Labs Research, Inc., Wheat Ridge, Colorado. Samples were graphite electrodes, $0.1 \mathrm{in}$. in diameter by $0.5 \mathrm{in}$. long ( $225 \mathrm{mg}$ ). A description of this technique is given in Ref. A-2.

\section{A.5.4. Sulfur Analysis}

Analyses for sulfur were conducted by iodometric titration as follows, One-gram samples of graphite were burned in oxygen to convert sulfur to sulfur dioxide. The sulfur dioxide was passed through a potassium iodide starch solution and was titrated with potassium iodate solution. The potassium iodate solution is standardized against samples of known sulfur content.

\section{A.5.5. Isotope Dilution Spark Source Mass Spectrometry (ORNL)}

Analyses by spark source mass spectrometry employing isotope dilution were conducted at ORNL. The spark source mass spectrometry technique is described in Ref. A-1. The method of isotope dilution is described in Ref. A-3. 
TABLE A-1

LOSSES RESULTING FROM ASHING

\begin{tabular}{|c|c|c|c|}
\hline Element & Percent Loss & Element & Percent Loss \\
\hline $\mathrm{Ag}$ & 80 & $\mathrm{Mn}$ & 20 \\
\hline $\mathrm{A} 1$ & 0 & Mo & 20 \\
\hline$B$ & 95 & $\mathrm{Na}$ & 20 \\
\hline $\mathrm{Ba}$ & 0 & $\mathrm{Nb}$ & 0 \\
\hline $\mathrm{Be}$ & 0 & $\mathrm{Nd}$ & 0 \\
\hline $\mathrm{Bi}$ & 40 & $\mathrm{Ni}$ & 20 \\
\hline $\mathrm{Ca}$ & 0 & $\mathrm{P}$ & 20 \\
\hline $\mathrm{Cd}$ & 80 & $\mathrm{~Pb}$ & 20 \\
\hline $\mathrm{Ce}$ & 0 & $\operatorname{Pr}$ & 0 \\
\hline Co & 20 & $\mathrm{Rb}$ & 20 \\
\hline $\mathrm{Cr}$ & 20 & $\mathrm{Sb}$ & 20 \\
\hline $\mathrm{Cs}$ & 50 & $\mathrm{Sc}$ & 0 \\
\hline $\mathrm{Cu}$ & 0 & Si & 20 \\
\hline Dy & 0 & $\mathrm{Sm}$ & 0 \\
\hline Er & 0 & Sn & 20 \\
\hline $\mathrm{Eu}$ & 0 & $\mathrm{Sr}$ & 0 \\
\hline $\mathrm{Fe}$ & 20 & $\mathrm{Ta}$ & 0 \\
\hline Gd & 0 & $\mathrm{~Tb}$ & 0 \\
\hline $\mathrm{Hf}$ & 0 & $\mathrm{Tì}$ & 0 \\
\hline Ho & 0 & $\mathrm{~T} 1$ & 100 \\
\hline In & 20 & $\mathrm{Tm}$ & 0 \\
\hline $\mathrm{K}$ & 20 & $\mathrm{y}$ & 80 \\
\hline $\mathrm{La}$ & 0 & W & 20 \\
\hline $\mathrm{Li}$ & 50 & $\mathrm{Yb}$ & 0 \\
\hline $\mathrm{Lu}$ & 0 & $\mathrm{Zn}$ & 40 \\
\hline $\mathrm{Mg}$ & 0 & $\mathrm{Zr}$ & 0 \\
\hline
\end{tabular}




\section{A.5.6. Atomic Absorption Analysis for Lithium}

Analyses for 1ithium by atomic absorption were conducted as follows. Ten-gram samples of graphite were oxidized (dissolved) in a mixture of fuming nitric acid and perchloric acid under reflux. The resultant solution was then analyzed for 1ithium on an atomic absorption spectrophotometer. Lithium was determined by comparison of the absorbance of the sample solution with the absorbance of standard lithium solutions.

REFERENCES

A-1. General Atomic unpublished data.

A-2. Brown, R., M.L. Jacobs, and H.E. Taylor, American Laboratory 4 (No. 11), 29 (1972).

A-3. Poulsen, P.J., R. Alvarez, and C.W. Mueller, Analytical Chemistry 42, 643 (1970). 


\section{APPENDIX B}

\section{TABULATED DATA}

Appendix $B$ contains complete sets of data for density and tensile properties, Poisson's ratio, thermal expansivity, thermal conductivity, and impurity content on $\mathrm{H}-451$ and $\mathrm{TS}-1240$ graphites. 
TABLE B-1

DENSITY AND TENSILE PROPERTIES OF H-451, LOT 2663 (a)

$\left[\log 5651-28(\right.$ GLCC 18$)$, whole $\log$ density $=1.77 \mathrm{~g} / \mathrm{cm}^{3}$

0.505-in.-diameter by 4.0-in.-long specimens]

\begin{tabular}{|c|c|c|c|c|c|}
\hline $\begin{array}{c}\text { GA } \\
\text { Specimen } \\
\text { No. } \\
\end{array}$ & $\begin{array}{l}\text { Location in } \\
\text { Log and } \\
\text { Orientation }\end{array}$ & $\begin{array}{r}\text { Density } \\
\left(\mathrm{g} / \mathrm{cm}^{3}\right)\end{array}$ & $\begin{array}{l}\text { Strain at } \\
\text { Fracture } \\
(\%) \\
\end{array}$ & $\begin{array}{l}\text { Ultimate } \\
\text { Strength } \\
\text { (psi) }\end{array}$ & $\begin{array}{r}\text { Modulus of } \\
\begin{array}{r}\text { Elasticity (b) } \\
\text { (psi) }\end{array}\end{array}$ \\
\hline $\begin{array}{r}5651-28-201 \\
-202 \\
-203 \\
-204 \\
-205 \\
-206 \\
-207 \\
-208 \\
-209 \\
-210 \\
-211 \\
-212 \\
-221 \\
-222 \\
-223 \\
-226 \\
-227 \\
-228 \\
-231 \\
-232 \\
-233 \\
-236 \\
-237 \\
-238 \\
-241 \\
-242 \\
-243 \\
-246 \\
-247 \\
-248 \\
-251 \\
-252 \\
-253 \\
-256\end{array}$ & MLC, axia1 & $\begin{array}{l}1.70 \\
1.74 \\
1.74 \\
1.75 \\
1.75 \\
1.74 \\
1.74 \\
1.73 \\
1.73 \\
1.75 \\
1.74 \\
1.74 \\
1.75 \\
1.75 \\
1.74 \\
1.69 \\
1.75 \\
1.73 \\
1.75 \\
1.75 \\
1.74 \\
1.65 \\
1.75 \\
1.74 \\
1.77 \\
1.74 \\
1.72 \\
1.75 \\
1.75 \\
1.75 \\
1.72 \\
1.74 \\
1.75 \\
1.73 \\
\end{array}$ & & & \\
\hline $\begin{array}{r}5651-28-10014 \\
-10015 \\
-10016 \\
-10017 \\
-10018 \\
-10019 \\
-10020\end{array}$ & 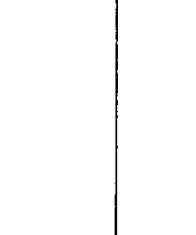 & & $\begin{array}{l}0.214 \\
0.206 \\
0.173 \\
0.194 \\
0.204 \\
0.200 \\
0.216 \\
\end{array}$ & $\begin{array}{l}1910 \\
1874 \\
1849 \\
1900 \\
1935 \\
1976 \\
1951 \\
\end{array}$ & $\begin{array}{l}1.32 \\
1.19 \\
1.39 \\
1.25 \\
1.19 \\
1.32 \\
1.14 \\
\end{array}$ \\
\hline $\begin{array}{l}\text { Mean } \\
\text { Std. Dev. }\end{array}$ & $\downarrow$ & $\begin{array}{l}1.74 \\
0.02\end{array}$ & $\begin{array}{l}0.201 \\
0.015\end{array}$ & $\begin{array}{r}1914 \\
44\end{array}$ & $\begin{array}{l}1.26 \\
0.09\end{array}$ \\
\hline $\begin{array}{r}5651-28-10011 \\
-10012 \\
-10013\end{array}$ & MLC, $\operatorname{radial}$ & & $\begin{array}{l}0.180 \\
0.120 \\
0.093 \\
\end{array}$ & $\begin{array}{r}1324 \\
952 \\
983 \\
\end{array}$ & $\begin{array}{l}1.00 \\
0.86 \\
0.96 \\
\end{array}$ \\
\hline $\begin{array}{l}\text { Mean } \\
\text { Std. Dev. }\end{array}$ & $f$ & & $\begin{array}{l}0.131 \\
0.045\end{array}$ & $\begin{array}{r}1086 \\
206\end{array}$ & $\begin{array}{l}0.94 \\
0.07\end{array}$ \\
\hline
\end{tabular}


TABLE B-1 (Continued)

\begin{tabular}{|c|c|c|c|c|c|}
\hline $\begin{array}{c}\text { GA } \\
\text { Specimen } \\
\text { No. }\end{array}$ & $\begin{array}{l}\text { Lucation in } \\
\text { Log and } \\
\text { orientation }\end{array}$ & $\begin{array}{l}\text { Density } \\
\left(\mathrm{g} / \mathrm{cm}^{3}\right)\end{array}$ & $\begin{array}{l}\text { Strain at } \\
\text { Fracture } \\
(\%)\end{array}$ & $\begin{array}{l}\text { Ultimate } \\
\text { Strength } \\
\quad(p s i)\end{array}$ & $\begin{array}{c}\text { Modulus of } \\
\text { Elasticity(b) } \\
\text { (psi) }\end{array}$ \\
\hline $\begin{array}{r}5651-28-224 \\
-225 \\
-229 \\
-230 \\
-234 \\
-235 \\
-239 \\
-240 \\
-244 \\
-245 \\
-249 \\
-250 \\
-254 \\
-255\end{array}$ & MLE, axial & $\begin{array}{l}1.72 \\
1.75 \\
1.74 \\
1.75 \\
1.74 \\
1.74 \\
1.74 \\
1.76 \\
1.75 \\
1.75 \\
1.73 \\
1.75 \\
1.74 \\
1.72 \\
\end{array}$ & & & \\
\hline $\begin{array}{r}5651-28-10024 \\
-10025 \\
-10026 \\
-10027 \\
-10028 \\
-10029 \\
-10030\end{array}$ & & & $\begin{array}{l}0.256 \\
0.237 \\
0.216 \\
0.193 \\
0.240 \\
0.244 \\
0.27 .1 \\
\end{array}$ & $\begin{array}{l}2251 \\
2190 \\
2215 \\
1920 \\
2231 \\
2256 \\
2245 \\
\end{array}$ & $\begin{array}{l}1.19 \\
1.19 \\
1.47 \\
1.25 \\
1.25 \\
1.32 \\
1.32 \\
\end{array}$ \\
\hline $\begin{array}{l}\text { Mean } \\
\text { Std. Dev. }\end{array}$ & $t$ & $\begin{array}{l}1.74 \\
0.01\end{array}$ & $\begin{array}{l}0.237 \\
0.026\end{array}$ & $\begin{array}{r}2215 \\
155\end{array}$ & $\begin{array}{l}1.28 \\
0.10\end{array}$ \\
\hline $\begin{array}{r}5651-28-10021 \\
-10022 \\
-10023\end{array}$ & MLE, radial & & $\begin{array}{l}0.147 \\
0.165 \\
0.092 \\
\end{array}$ & $\begin{array}{r}1243 \\
1334 \\
876 \\
\end{array}$ & $\begin{array}{l}0.93 \\
0.93 \\
0.93 \\
\end{array}$ \\
\hline $\begin{array}{l}\text { Mean } \\
\text { Std. Dev. }\end{array}$ & 1 & & $\begin{array}{l}0.135 \\
0.038\end{array}$ & $\begin{array}{r}1151 \\
242\end{array}$ & $\begin{array}{l}0.93 \\
0.00\end{array}$ \\
\hline $\begin{array}{r}5651-28-10005 \\
-10006 \\
-10007 \\
-10008 \\
-10009 \\
-10010\end{array}$ & EC, axial & & $\begin{array}{l}0.198 \\
0.158 \\
0.216 \\
0.182 \\
0.195 \\
0.229 \\
\end{array}$ & $\begin{array}{l}1910 \\
1706 \\
2012 \\
1910 \\
1920 \\
1874 \\
\end{array}$ & $\begin{array}{l}1.25 \\
1.32 \\
1.25 \\
1.32 \\
1.19 \\
1.00 \\
\end{array}$ \\
\hline $\begin{array}{l}\text { Mean } \\
\text { Std. Dev. }\end{array}$ & $f$ & & $\begin{array}{l}0.196 \\
0.025\end{array}$ & $\begin{array}{r}1889 \\
101\end{array}$ & $\begin{array}{l}1.22 \\
0.12\end{array}$ \\
\hline $\begin{array}{r}5651-28-10001 \\
-10002 \\
-10003\end{array}$ & EC, radial & & $\begin{array}{l}0.210 \\
0.176 \\
0.132 \\
\end{array}$ & $\begin{array}{l}1564 \\
1411 \\
1212 \\
\end{array}$ & $\begin{array}{l}0.96 \\
0.93 \\
1.00 \\
\end{array}$ \\
\hline $\begin{array}{l}\text { Mean } \\
\text { Std. Dev. }\end{array}$ & $t$ & & $\begin{array}{l}0.173 \\
0.039\end{array}$ & $\begin{array}{r}1396 \\
177\end{array}$ & $\begin{array}{l}0.96 \\
0.04\end{array}$ \\
\hline
\end{tabular}

(a) Measured at GLCC.

(b) Chord modulus between 250 and 500 psi. 
TABLE B-2

TENSILE PROPERTIES OF H-451, LOT 266

[Log 5651-28 (GLCC 18), whole 10 g density $=1.77 \mathrm{~g} / \mathrm{cm}^{3}(\mathrm{a})$

0.250-in.-diameter by 0.90-1n.-long specimens]

\begin{tabular}{|c|c|c|c|c|}
\hline $\begin{array}{l}\text { GA } \\
\text { Specimen } \\
\text { No. }\end{array}$ & $\begin{array}{l}\text { Location in } \\
\text { Log and } \\
\text { Orientation }\end{array}$ & $\begin{array}{l}\text { Strain at } \\
\text { Fracture } \\
\quad(\%)\end{array}$ & $\begin{array}{l}\text { U1timate } \\
\text { Strength } \\
\quad \text { (psi) }\end{array}$ & $\begin{array}{c}\text { Modulus of } \\
\text { Elasticity(b) } \\
\text { (psi) }\end{array}$ \\
\hline $\begin{array}{r}5651-28-10042 \\
-10043 \\
-10044 \\
-10045 \\
-10046 \\
-10047 \\
-10048\end{array}$ & MLC, axial & $\begin{array}{l}0.202 \\
0.225 \\
0.206 \\
0.238 \\
0.225 \\
0.220 \\
0.154 \\
\end{array}$ & $\begin{array}{l}1681 \\
1752 \\
1803 \\
1966 \\
1772 \\
1874 \\
1721 \\
\end{array}$ & $\begin{array}{l}1.00 \\
1.11 \\
1.18 \\
1.00 \\
1.05 \\
1.33 \\
1.18 \\
\end{array}$ \\
\hline $\begin{array}{l}\text { Mean } \\
\text { Std. Dev. }\end{array}$ & 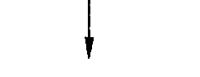 & $\begin{array}{l}0.210 \\
0.028\end{array}$ & $\begin{array}{r}1796 \\
97\end{array}$ & $\begin{array}{l}1.12 \\
0.12\end{array}$ \\
\hline $\begin{array}{r}5651-28-10039 \\
-10040 \\
-10041\end{array}$ & MLC, radial & $\begin{array}{l}0.206 \\
0.240 \\
0.245 \\
\end{array}$ & $\begin{array}{l}1487 \\
1548 \\
1640 \\
\end{array}$ & $\begin{array}{l}0.83 \\
0.83 \\
0.95 \\
\end{array}$ \\
\hline $\begin{array}{l}\text { Mean. } \\
\text { Std. Dev. }\end{array}$ & $\downarrow$ & $\begin{array}{l}0.230 \\
0.021\end{array}$ & $\begin{array}{r}1558 \\
77\end{array}$ & $\begin{array}{l}0.87 \\
0.07\end{array}$ \\
\hline $\begin{array}{r}5651-28-10052 \\
-10053 \\
-10054 \\
-10055 \\
-10056 \\
-10057 \\
-10058\end{array}$ & MLE, axial & $\begin{array}{l}0.294 \\
0.253 \\
0.272 \\
0.280 \\
0.272 \\
0.276 \\
0.303 \\
\end{array}$ & $\begin{array}{l}2322 \\
2118 \\
2220 \\
2220 \\
2200 \\
2241 \\
2404 \\
\end{array}$ & $\begin{array}{l}1.11 \\
1.05 \\
1.18 \\
1.11 \\
1.00 \\
1.18 \\
1.11 \\
\end{array}$ \\
\hline $\begin{array}{l}\text { Mean } \\
\text { Std. Dev. }\end{array}$ & 1 & $\begin{array}{l}0.279 \\
0.016\end{array}$ & $\begin{array}{r}2246 \\
92\end{array}$ & $\begin{array}{l}1.11 \\
0.07\end{array}$ \\
\hline $\begin{array}{r}5651-28-10049 \\
-10050 \\
-10051\end{array}$ & MLE, radial & $\begin{array}{l}0.312 \\
0.125 \\
0.247 \\
\end{array}$ & $\begin{array}{l}2037 \\
1147 \\
1732 \\
\end{array}$ & $\begin{array}{l}0.91 \\
0.95 \\
1.00 \\
\end{array}$ \\
\hline $\begin{array}{l}\text { Mean } \\
\text { Std. Dev. }\end{array}$ & 1 & $\begin{array}{l}0.228 \\
0.095\end{array}$ & $\begin{array}{r}1637 \\
456\end{array}$ & $\begin{array}{l}0.95 \\
0.05\end{array}$ \\
\hline $\begin{array}{r}5651-28-10034 \\
-10035 \\
-10036 \\
-10037 \\
-10038 \\
-10059 \\
-10060\end{array}$ & $\left.\right|^{E C,}$ & $\begin{array}{l}0.233 \\
0.231 \\
0.179 \\
0.163 \\
0.257 \\
0.171 \\
0.212 \\
\end{array}$ & $\begin{array}{l}1691 \\
1670 \\
1732 \\
1548 \\
1966 \\
1283 \\
1711 \\
\end{array}$ & $\begin{array}{l}1.00 \\
1.00 \\
1.25 \\
1.00 \\
1.00 \\
\text { N.A. } \\
\text { N.A. } \\
\end{array}$ \\
\hline $\begin{array}{l}\text { Mean } \\
\text { Std. Dev. }\end{array}$ & 1 & $\begin{array}{l}0.207 \\
0.036\end{array}$ & $\begin{array}{r}1657 \\
207\end{array}$ & $\begin{array}{l}1.05 \\
0.11\end{array}$ \\
\hline $\begin{array}{r}5651-28-10031 \\
-10032 \\
-10033\end{array}$ & $E C, \operatorname{radial}$ & $\begin{array}{l}0.348 \\
0.225 \\
0.235 \\
\end{array}$ & $\begin{array}{l}2384 \\
2078 \\
1691 \\
\end{array}$ & $\begin{array}{l}1.00 \\
1.25 \\
0.87 \\
\end{array}$ \\
\hline $\begin{array}{l}\text { Mean } \\
\text { Std. Dev. }\end{array}$ & $\gamma$ & $\begin{array}{l}0.269 \\
0.068\end{array}$ & $\begin{array}{r}2051 \\
347\end{array}$ & $\begin{array}{l}1.05 \\
0.19\end{array}$ \\
\hline
\end{tabular}

(a) Measured at GLCC.

(b) Chord modulus between 250 and 500 psi. 
TABLE B-3

DENSITY AND TENSILE PROPERTIES OF H-451, LOT 266

[Log 5651-58 (GLCC unknown), 0.505-in.-diameter by 4.0-in.-long specimens]

\begin{tabular}{|c|c|c|c|c|c|}
\hline $\begin{array}{c}\text { GA } \\
\text { Specimen } \\
\text { No. }\end{array}$ & $\begin{array}{l}\text { Location in } \\
\text { Log and } \\
\text { Orientation }\end{array}$ & $\begin{array}{r}\text { Density } \\
\left(\mathrm{g} / \mathrm{cm}^{3}\right) \\
\end{array}$ & $\begin{array}{l}\text { Strain at } \\
\text { Fracture } \\
\quad(\%) \\
\end{array}$ & $\begin{array}{l}\text { Ultimate } \\
\text { Strength } \\
\text { (psi) }\end{array}$ & $\begin{array}{r}\text { Modulus of } \\
\text { Elasticity(a) } \\
\text { (psi) }\end{array}$ \\
\hline $\begin{array}{r}5651-58-1 \\
-2 \\
-3 \\
-4 \\
-5 \\
-6 \\
-7 \\
-8 \\
-9 \\
-10 \\
-11 \\
-12 \\
-13 \\
-14 \\
-15 \\
-16 \\
-17 \\
-18 \\
-19\end{array}$ & MLC, axial & $\begin{array}{l}1.73 \\
1.73 \\
1.73 \\
1.72 \\
1.72 \\
1.72 \\
1.73 \\
1.73 \\
1.74 \\
1.73 \\
1.73 \\
1.74 \\
1.73 \\
1.73 \\
1.73 \\
1.73 \\
1.73 \\
1.73 \\
1.73 \\
\end{array}$ & & & \\
\hline $\begin{array}{r}5651-58-10061 \\
-10062 \\
-10063 \\
-10064 \\
-10065 \\
-10066 \\
-10067 \\
-10068\end{array}$ & & & $\begin{array}{l}0.191 \\
0.168 \\
0.174 \\
0.166 \\
0.172 \\
0.130 \\
0.204 \\
0.200 \\
\end{array}$ & $\begin{array}{l}1681 \\
1477 \\
1630 \\
1630 \\
1620 \\
1263 \\
1859 \\
2088 \\
\end{array}$ & $\begin{array}{l}1.14 \\
1.09 \\
1.25 \\
1.19 \\
1.09 \\
1.14 \\
1.25 \\
1.47 \\
\end{array}$ \\
\hline $\begin{array}{l}\text { Mean } \\
\text { Std. Dev. }\end{array}$ & 1 & $\begin{array}{l}1.73 \\
0.01\end{array}$ & $\begin{array}{l}0.176 \\
0.024\end{array}$ & $\begin{array}{r}1656 \\
244\end{array}$ & $\begin{array}{l}1.20 \\
0.13\end{array}$ \\
\hline $\begin{array}{r}5651-58-10069 \\
-10070 \\
-10071 \\
-10072 \\
-10073 \\
-10074 \\
-10075\end{array}$ & MLC, radial & & $\begin{array}{l}0.171 \\
0.155 \\
0.120 \\
0.137 \\
0.185 \\
0.205 \\
0.220 \\
\end{array}$ & $\begin{array}{l}1289 \\
1273 \\
1146 \\
1070 \\
1462 \\
1589 \\
1538 \\
\end{array}$ & $\begin{array}{l}0.96 \\
0.96 \\
1.04 \\
0.64 \\
0.93 \\
1.04 \\
0.93 \\
\end{array}$ \\
\hline $\begin{array}{l}\text { Mean } \\
\text { Std. Dev. }\end{array}$ & $\downarrow$ & & $\begin{array}{l}0.170 \\
0.036\end{array}$ & $\begin{array}{r}1338 \\
197\end{array}$ & $\begin{array}{l}0.93 \\
0.14\end{array}$ \\
\hline $\begin{array}{r}5651-58-21 \\
-22 \\
-23 \\
-24 \\
-25 \\
-26 \\
-27 \\
-28 \\
-29\end{array}$ & MLE, axial & $\begin{array}{l}1.73 \\
1.73 \\
1.73 \\
1.73 \\
1.73 \\
1.73 \\
1.73 \\
1.73 \\
1.73 \\
\end{array}$ & & & \\
\hline $\begin{array}{l}\text { Mean } \\
\text { Std. Dev. }\end{array}$ & 1 & $\begin{array}{l}1.73 \\
0.00\end{array}$ & & & \\
\hline $\begin{array}{r}5651-58-10076 \\
-10077 \\
-10078 \\
-10079 \\
-10080 \\
-10081 \\
-10082\end{array}$ & MLE, radial & & $\begin{array}{l}0.221 \\
0.150 \\
0.160 \\
0.190 \\
0.250 \\
0.225 \\
0.210 \\
\end{array}$ & $\begin{array}{l}1716 \\
1304 \\
1401 \\
1492 \\
1905 \\
1716 \\
1665 \\
\end{array}$ & $\begin{array}{l}0.93 \\
0.96 \\
0.93 \\
0.93 \\
1.00 \\
0.96 \\
0.96 \\
\end{array}$ \\
\hline $\begin{array}{l}\text { Mean } \\
\text { Std. Dev. }\end{array}$ & 1 & & $\begin{array}{l}0.210 \\
0.036\end{array}$ & $\begin{array}{r}1600 \\
209\end{array}$ & $\begin{array}{l}0.95 \\
0.03\end{array}$ \\
\hline
\end{tabular}

(a) Chord modulus between 250 and 500 psi. 
TABLE B-4

DENSITY AND TENSILE STRENGTH OF H-451, LOT 408

[ Log 5651-90 (GLCC 48), whole log density $=1.75 \mathrm{~g} / \mathrm{cm}^{3}{ }^{(a)}$, 0.250-in.-diameter by 0.9-in.-long specimens]

\begin{tabular}{|c|c|c|c|}
\hline $\begin{array}{c}\text { GA } \\
\text { Specimen } \\
\text { No. } \\
\end{array}$ & $\begin{array}{l}\text { Location in } \\
\text { Log and } \\
\text { Orientation }\end{array}$ & $\begin{array}{l}\text { Density } \\
\left(\mathrm{g} / \mathrm{cm}^{3}\right)\end{array}$ & $\begin{array}{l}\text { Ultimate } \\
\text { Strength } \\
\quad \text { (psi) }\end{array}$ \\
\hline $\begin{array}{l}2 \mathrm{AC}-300-\mathrm{A} 1 \\
2 \mathrm{AC}-301-\mathrm{A} 1 \\
2 \mathrm{AC}-302-\mathrm{A} 1 \\
2 \mathrm{AC}-303-\mathrm{A} 1 \\
2 \mathrm{AC}-304-\mathrm{A} 1 \\
2 \mathrm{AC}-305-\mathrm{A} 1 \\
2 \mathrm{AC}-306-\mathrm{A} 1 \\
2 \mathrm{AC}-307-\mathrm{A} 1 \\
2 \mathrm{AC}-308-\mathrm{A} 1 \\
2 \mathrm{AC}-309-\mathrm{A} 1 \\
2 \mathrm{AC}-310-\mathrm{A} 1 \\
2 \mathrm{AC}-311-\mathrm{A} 1 \\
2 \mathrm{AC}-312-\mathrm{A} 1 \\
2 \mathrm{AC}-313-\mathrm{A} 1 \\
2 \mathrm{AC}-314-\mathrm{A} 1 \\
2 \mathrm{AC}-315-\mathrm{A} 1 \\
2 \mathrm{AC}-316-\mathrm{A} 1 \\
2 \mathrm{AC}-317-\mathrm{A} 1\end{array}$ & MLC, radial & $\begin{array}{l}1.742 \\
1.734 \\
1.735 \\
1.741 \\
1.732 \\
1.736 \\
1.730 \\
1.736 \\
1.731 \\
1.726 \\
1.735 \\
1.736 \\
1.731 \\
1.734 \\
1.741 \\
1.739 \\
1.744 \\
1.738\end{array}$ & $\begin{array}{l}2212 \\
1418 \\
1862 \\
2245 \\
1603 \\
2065 \\
2048 \\
1219 \\
1076 \\
1018 \\
1792 \\
1424 \\
1589 \\
1365 \\
1813 \\
1877 \\
2283 \\
816\end{array}$ \\
\hline $\begin{array}{l}2 \mathrm{BC}-300-\mathrm{A} 1 \\
2 \mathrm{BC}-301-\mathrm{A} 1 \\
2 \mathrm{BC}-302-\mathrm{A} 1 \\
2 \mathrm{BC}-303-\mathrm{A} 1 \\
2 \mathrm{BC}-304-\mathrm{A} 1 \\
2 \mathrm{BC}-305-\mathrm{A} 1 \\
2 \mathrm{BC}-306-\mathrm{A} 1 \\
2 \mathrm{BC}-307-\mathrm{A} 1 \\
2 \mathrm{BC}-308-\mathrm{A} 1 \\
2 \mathrm{BC}-309-\mathrm{A} 1 \\
2 \mathrm{BC}-310-\mathrm{A} 1 \\
2 \mathrm{BC}-311-\mathrm{A} 1 \\
2 \mathrm{BC}-312-\mathrm{A} 1 \\
2 \mathrm{BC}-313-\mathrm{A} 1 \\
2 \mathrm{BC}-314-\mathrm{A} 1 \\
2 \mathrm{BC}-315-\mathrm{A} 1 \\
2 \mathrm{BC}-316-\mathrm{A} 1 \\
2 \mathrm{BC}-317-\mathrm{A} 1 \\
2 \mathrm{BC}-318-\mathrm{A} 1 \\
2 \mathrm{BC}-319-\mathrm{A} 1\end{array}$ & & $\begin{array}{l}1.734 \\
1.735 \\
1.733 \\
1.727 \\
1.732 \\
1.724 \\
1.730 \\
1.726 \\
1.726 \\
1.734 \\
1.730 \\
1.736 \\
1.741 \\
1.730 \\
1.731 \\
1.732 \\
1.735 \\
1.735 \\
1.729 \\
1.736 \\
\end{array}$ & $\begin{array}{l}1381 \\
1951 \\
2131 \\
1095 \\
2028 \\
1721 \\
1583 \\
1865 \\
1786 \\
1803 \\
2193 \\
2453 \\
2234 \\
2249 \\
2310 \\
2045 \\
1823 \\
2026 \\
1619 \\
2006 \\
\end{array}$ \\
\hline $\begin{array}{l}\text { Mean } \\
\text { Std. Dev. }\end{array}$ & $\downarrow$ & $\begin{array}{l}1.734 \\
0.005\end{array}$ & $\begin{array}{r}1783 \\
399\end{array}$ \\
\hline
\end{tabular}


TABLE B-4 (Continued)

\begin{tabular}{|c|c|c|c|}
\hline $\begin{array}{l}\text { GA } \\
\text { Specimen } \\
\text { No. }\end{array}$ & $\begin{array}{l}\text { Location in } \\
\text { Log and } \\
\text { Orientation }\end{array}$ & $\begin{array}{l}\text { Density } \\
\left(\mathrm{g} / \mathrm{cm}^{3}\right)\end{array}$ & $\begin{array}{l}\text { Ultimate } \\
\text { Strength } \\
\text { (psi) }\end{array}$ \\
\hline $\begin{array}{l}2 \mathrm{AC}-1-\mathrm{A} 1 \\
2 \mathrm{AC}-1-\mathrm{A} 2 \\
2 \mathrm{AC}-2-\mathrm{A} 1 \\
2 \mathrm{AC}-2-\mathrm{A} 2 \\
2 \mathrm{AC}-3-\mathrm{A} 1 \\
2 \mathrm{AC}-3-\mathrm{A} 2 \\
2 \mathrm{AC}-4-\mathrm{A} 1 \\
2 \mathrm{AC}-4-\mathrm{A} 2 \\
2 \mathrm{AC}-5-\mathrm{A} 1 \\
2 \mathrm{AC}-5-\mathrm{A} 2 \\
2 \mathrm{AC}-6-\mathrm{A} 1 \\
2 \mathrm{AC}-6-\mathrm{A} 2 \\
2 \mathrm{AC}-7-\mathrm{A} 1 \\
2 \mathrm{AC}-7-\mathrm{A} 2 \\
2 \mathrm{AC}-8-\mathrm{A} 1 \\
2 \mathrm{AC}-8-\mathrm{A} 2 \\
2 \mathrm{AC}-9-\mathrm{A} 1 \\
2 \mathrm{AC}-9-\mathrm{A} 2 \\
2 \mathrm{AC}-10-\mathrm{A} 1 \\
2 \mathrm{AC}-10-\mathrm{A} 2\end{array}$ & MLC, axial & $\begin{array}{l}1.733 \\
1.742 \\
1.735 \\
1.727 \\
1.737 \\
1.729 \\
1.733 \\
1.730 \\
1.726 \\
1.725 \\
1.717 \\
1.728 \\
1.730 \\
1.741 \\
1.729 \\
1.722 \\
1.727 \\
\text { No core } \\
1.720 \\
1.724\end{array}$ & $\begin{array}{l}1344 \\
2132 \\
2073 \\
2153 \\
2417 \\
2297 \\
2341 \\
1914 \\
2073 \\
2186 \\
2188 \\
1747 \\
1943 \\
2205 \\
2147 \\
2230 \\
2208 \\
-- \\
2194 \\
1806\end{array}$ \\
\hline $\begin{array}{l}2 \mathrm{BC}-1-\mathrm{A} 1 \\
2 \mathrm{BC}-1-\mathrm{A} 2 \\
2 \mathrm{BC}-2-\mathrm{A} 1 \\
2 \mathrm{BC}-2-\mathrm{A} 2 \\
2 \mathrm{BC}-3-\mathrm{A} 1 \\
2 \mathrm{BC}-3-\mathrm{A} 2 \\
2 \mathrm{BC}-4-\mathrm{A} 1 \\
2 \mathrm{BC}-4-\mathrm{A} 2 \\
2 \mathrm{BC}-5-\mathrm{A} 1 \\
2 \mathrm{BC}-5-\mathrm{A} 2 \\
2 \mathrm{BC}-6-\mathrm{A} 1 \\
2 \mathrm{BC}-6-\mathrm{A} 2 \\
2 \mathrm{BC}-7-\mathrm{A} 1 \\
2 \mathrm{BC}-7-\mathrm{A} 2 \\
2 \mathrm{BC}-8-\mathrm{A} 1 \\
2 \mathrm{BC}-8-\mathrm{A} 2 \\
2 \mathrm{BC}-9-\mathrm{A} 1 \\
2 \mathrm{BC}-9-\mathrm{A} 2 \\
2 \mathrm{BC}-10-\mathrm{A} 1 \\
2 \mathrm{BC}-10-\mathrm{A} 2\end{array}$ & & $\begin{array}{l}1.740 \\
1.727 \\
1.737 \\
1.732 \\
1.738 \\
1.734 \\
1.731 \\
1.738 \\
1.716 \\
1.723 \\
1.738 \\
1.731 \\
1.728 \\
1.731 \\
1.736 \\
1.735 \\
1.736 \\
1.739 \\
1.742 \\
1.744 \\
\end{array}$ & $\begin{array}{l}2490 \\
2245 \\
2451 \\
2208 \\
2249 \\
2193 \\
2109 \\
2267 \\
1828 \\
2171 \\
2075 \\
2169 \\
1847 \\
2111 \\
2374 \\
2334 \\
2147 \\
2271 \\
2600 \\
2358 \\
\end{array}$ \\
\hline $\begin{array}{l}\text { Mean } \\
\text { Std. Dev. }\end{array}$ & $V$ & $\begin{array}{l}1.732 \\
0.007\end{array}$ & $\begin{array}{r}2168 \\
185\end{array}$ \\
\hline
\end{tabular}


TABLE B-4 (Continued)

\begin{tabular}{|c|c|c|c|}
\hline $\begin{array}{c}\text { GA } \\
\text { Specimen } \\
\text { No. }\end{array}$ & $\begin{array}{l}\text { Location in } \\
\text { Log and } \\
\text { Orientation }\end{array}$ & $\begin{array}{l}\text { Density } \\
\left(\mathrm{g} / \mathrm{cm}^{3}\right)\end{array}$ & $\begin{array}{l}\text { U1timate } \\
\text { Strength } \\
\quad \text { (psi) }\end{array}$ \\
\hline $\begin{array}{l}2 \mathrm{AE}-300-\mathrm{A} 1 \\
2 \mathrm{AE}-301-\mathrm{A} 1 \\
2 \mathrm{AE}-302-\mathrm{A} 1 \\
2 \mathrm{AE}-303-\mathrm{A} 1 \\
2 \mathrm{AE}-304-\mathrm{A} 1 \\
2 \mathrm{AE}-305-\mathrm{A} 1 \\
2 \mathrm{AE}-306-\mathrm{A} 1 \\
2 \mathrm{AE}-307-\mathrm{A} 1 \\
2 \mathrm{AE}-308-\mathrm{A} 1 \\
2 \mathrm{AE}-309-\mathrm{A} 1 \\
2 \mathrm{AE}-310-\mathrm{A} 1 \\
2 \mathrm{AE}-311-\mathrm{A} 1 \\
2 \mathrm{AE}-312-\mathrm{A} 1 \\
2 \mathrm{AE}-313-\mathrm{A} 1 \\
2 \mathrm{AE}-314-\mathrm{A} 1 \\
2 \mathrm{AE}-315-\mathrm{A} 1 \\
2 \mathrm{AE}-316-\mathrm{A} 1 \\
2 \mathrm{AE}-317-\mathrm{A} 1 \\
2 \mathrm{AE}-318-\mathrm{A} 1 \\
2 \mathrm{AE}-319-\mathrm{A} 1 \\
2 \mathrm{AE}-320-\mathrm{A} 1 \\
2 \mathrm{AE}-321-\mathrm{A} 1 \\
2 \mathrm{AE}-322-\mathrm{A} 1 \\
2 \mathrm{AE}-323-\mathrm{A} 1\end{array}$ & MLE, radial & $\begin{array}{l}1.740 \\
1.730 \\
1.744 \\
1.733 \\
1.738 \\
1.738 \\
1.737 \\
1.740 \\
1.740 \\
1.735 \\
1.732 \\
1.735 \\
1.736 \\
1.735 \\
1.734 \\
1.738 \\
1.729 \\
1.738 \\
1.739 \\
1.732 \\
1.738 \\
1.739 \\
1.727 \\
1.736\end{array}$ & $\begin{array}{l}2565 \\
2164 \\
2613 \\
1764 \\
2212 \\
2132 \\
2411 \\
2537 \\
2374 \\
2559 \\
2796 \\
2573 \\
2029 \\
2129 \\
2223 \\
2534 \\
1909 \\
2173 \\
1851 \\
2195 \\
2356 \\
1604 \\
1768 \\
1607\end{array}$ \\
\hline $\begin{array}{l}2 \mathrm{BE}-300-\mathrm{A} 1 \\
2 \mathrm{BE}-301-\mathrm{A} 1 \\
2 \mathrm{BE}-302-\mathrm{A} 1 \\
2 \mathrm{BE}-303-\mathrm{A} 1 \\
2 \mathrm{BE}-304-\mathrm{A} 1 \\
2 \mathrm{BE}-305-\mathrm{A} 1 \\
2 \mathrm{BE}-306-\mathrm{A} 1 \\
2 \mathrm{BE}-307-\mathrm{A} 1 \\
2 \mathrm{BE}-308-\mathrm{A} 1 \\
2 \mathrm{BE}-309-\mathrm{A} 1 \\
2 \mathrm{BE}-310-\mathrm{A} 1 \\
2 \mathrm{BE}-311-\mathrm{A} 1 \\
2 \mathrm{BE}-312-\mathrm{A} 1 \\
2 \mathrm{BE}-313-\mathrm{A} 1 \\
2 \mathrm{BE}-314-\mathrm{A} 1 \\
2 \mathrm{BE}-315-\mathrm{A} 1 \\
2 \mathrm{BE}-316-\mathrm{A} 1 \\
2 \mathrm{BE}-317-\mathrm{A} 1 \\
2 \mathrm{BE}-318-\mathrm{A} 1 \\
2 \mathrm{BE}-319-\mathrm{A} 1 \\
2 \mathrm{BE}-320-\mathrm{A} 1 \\
2 \mathrm{BE}-321-\mathrm{A} 1 \\
2 \mathrm{BE}-322-\mathrm{A} 1 \\
2 \mathrm{BE}-323-\mathrm{A} 1\end{array}$ & & $\begin{array}{l}1.739 \\
1.741 \\
1.751 \\
1.736 \\
1.710 \\
1.744 \\
1.749 \\
1.745 \\
1.749 \\
1.744 \\
1.749 \\
1.743 \\
1.736 \\
1.731 \\
1.739 \\
1.733 \\
1.737 \\
1.733 \\
1.741 \\
1.734 \\
1.742 \\
1.737 \\
1.739 \\
1.730\end{array}$ & $\begin{array}{l}2065 \\
1867 \\
2370 \\
2524 \\
2232 \\
2229 \\
2431 \\
2065 \\
2427 \\
2184 \\
2308 \\
2109 \\
2267 \\
2112 \\
2254 \\
2193 \\
2170 \\
2252 \\
2232 \\
2087 \\
1763 \\
2330 \\
2271 \\
2313\end{array}$ \\
\hline $\begin{array}{l}\text { Mean } \\
\text { Std. Dev. }\end{array}$ & 1 & $\begin{array}{l}1.737 \\
0.007\end{array}$ & $\begin{array}{r}2203 \\
260\end{array}$ \\
\hline
\end{tabular}


TABLE B-4 (Continued)

\begin{tabular}{|c|c|c|c|}
\hline $\begin{array}{c}\text { GA } \\
\text { Specimen } \\
\text { No. }\end{array}$ & $\begin{array}{l}\text { Location in } \\
\text { Log and } \\
\text { Orientation }\end{array}$ & $\begin{array}{l}\text { Density } \\
\left(\mathrm{g} / \mathrm{cm}^{3}\right)\end{array}$ & $\begin{array}{l}\text { Ultimate } \\
\text { Strength } \\
\quad \text { (psi) }\end{array}$ \\
\hline $\begin{array}{l}2 \mathrm{AE}-1-\mathrm{A} 1 \\
2 \mathrm{AE}-1-\mathrm{A} 2 \\
2 \mathrm{AE}-2-\mathrm{A} 1 \\
2 \mathrm{AE}-2-\mathrm{A} 2 \\
2 \mathrm{AE}-3-\mathrm{A} 1 \\
2 \mathrm{AE}-3-\mathrm{A} 2 \\
2 \mathrm{AE}-4-\mathrm{A} 1 \\
2 \mathrm{AE}-4-\mathrm{A} 2 \\
2 \mathrm{AE}-5-\mathrm{A} 1 \\
2 \mathrm{AE}-5-\mathrm{A} 2 \\
2 \mathrm{AE}-6-\mathrm{A} 1 \\
2 \mathrm{AE}-6-\mathrm{A} 2 \\
2 \mathrm{AE}-7-\mathrm{A} 1 \\
2 \mathrm{AE}-7-\mathrm{A} 2 \\
2 \mathrm{AE}-8-\mathrm{A} 1 \\
2 \mathrm{AE}-8-\mathrm{A} 2 \\
2 \mathrm{AE}-9-\mathrm{A} 1 \\
2 \mathrm{AE}-9-\mathrm{A} 2 \\
2 \mathrm{AE}-10-\mathrm{A} 1 \\
2 \mathrm{AE}-10-\mathrm{A} 2 \\
2 \mathrm{AE}-11-\mathrm{A} 1 \\
2 \mathrm{AE}-12-\mathrm{A} 1\end{array}$ & MLE, axia1 & $\begin{array}{l}1.732 \\
1.739 \\
1.734 \\
1.732 \\
1.739 \\
1.750 \\
1.738 \\
1.734 \\
1.739 \\
1.735 \\
1.736 \\
1.734 \\
1.737 \\
1.729 \\
1.730 \\
1.735 \\
1.741 \\
1.737 \\
1.744 \\
1.742 \\
1.732 \\
1.738\end{array}$ & $\begin{array}{l}2502 \\
2782 \\
2435 \\
2417 \\
2443 \\
2802 \\
2839 \\
2577 \\
2396 \\
1970 \\
2223 \\
2405 \\
2526 \\
2567 \\
2330 \\
2284 \\
2618 \\
2365 \\
2611 \\
2683 \\
2376 \\
2724\end{array}$ \\
\hline $\begin{array}{l}2 \mathrm{BE}-1-\mathrm{A} 1 \\
2 \mathrm{BE}-2-\mathrm{A} 1 \\
2 \mathrm{BE}-3-\mathrm{A} 1 \\
2 \mathrm{BE}-4-\mathrm{A} 1 \\
2 \mathrm{BE}-5-\mathrm{A} 1 \\
2 \mathrm{BE}-6-\mathrm{A} 1 \\
2 \mathrm{BE}-7-\mathrm{A} 1 \\
2 \mathrm{BE}-8-\mathrm{A} 1 \\
2 \mathrm{BE}-9-\mathrm{A} 1 \\
2 \mathrm{BE}-10-\mathrm{A} 1 \\
2 \mathrm{BE}-11-\mathrm{A} 1 \\
2 \mathrm{BE}-12-\mathrm{A} 1 \\
2 \mathrm{BE}-13-\mathrm{A} 1 \\
2 \mathrm{BE}-14-\mathrm{A} 1 \\
2 \mathrm{BE}-15-\mathrm{A} 1 \\
2 \mathrm{BE}-16-\mathrm{A} 1 \\
2 \mathrm{BE}-17-\mathrm{A} 1 \\
2 \mathrm{BE}-18-\mathrm{A} 1 \\
2 \mathrm{BE}-19-\mathrm{A} 1 \\
2 \mathrm{BE}-20-\mathrm{A} 1 \\
2 \mathrm{BE}-21-\mathrm{A} 1 \\
2 \mathrm{BE}-22-\mathrm{A} 1 \\
2 \mathrm{BE}-23-\mathrm{A} 1 \\
2 \mathrm{BE}-24-\mathrm{A} 1\end{array}$ & & $\begin{array}{l}1.748 \\
1.745 \\
1.743 \\
1.748 \\
1.748 \\
1.753 \\
1.738 \\
1.752 \\
1.739 \\
1.745 \\
1.745 \\
1.744 \\
1.745 \\
1.738 \\
1.751 \\
1.736 \\
1.736 \\
1.740 \\
1.731 \\
1.743 \\
1.732 \\
1.732 \\
1.735 \\
1.731\end{array}$ & $\begin{array}{l}3095 \\
3054 \\
2586 \\
2843 \\
3084 \\
2938 \\
2663 \\
2807 \\
2832 \\
2791 \\
2929 \\
2884 \\
2642 \\
2579 \\
2681 \\
2618 \\
2841 \\
2575 \\
2695 \\
2998 \\
2431 \\
2571 \\
2368 \\
3019\end{array}$ \\
\hline $\begin{array}{l}\text { Mean } \\
\text { Std. Dev. }\end{array}$ & $V$ & $\begin{array}{l}1.739 \\
0.006\end{array}$ & $\begin{array}{r}2628 \\
243\end{array}$ \\
\hline
\end{tabular}


TABLE B-4 (Continued)

\begin{tabular}{|c|c|c|c|}
\hline $\begin{array}{l}\text { GA } \\
\text { Specimen } \\
\text { No. }\end{array}$ & $\begin{array}{l}\text { Location in } \\
\text { Log and } \\
\text { Orientation }\end{array}$ & $\begin{array}{l}\text { Density } \\
\left(\mathrm{g} / \mathrm{cm}^{3}\right)\end{array}$ & $\begin{array}{l}\text { Ultimate } \\
\text { Strength } \\
\quad \text { (psi) }\end{array}$ \\
\hline $\begin{array}{l}\mathrm{C}-300-\mathrm{A} 1 \\
\mathrm{C}-301-\mathrm{A} 1 \\
\mathrm{C}-302-\mathrm{A} 1 \\
\mathrm{C}-303-\mathrm{A} 1 \\
\mathrm{C}-304-\mathrm{A} 1 \\
\mathrm{C}-305-\mathrm{A} 1 \\
\mathrm{C}-306-\mathrm{A} 1 \\
\mathrm{C}-307-\mathrm{A} 1 \\
\mathrm{C}-308-\mathrm{A} 1 \\
\mathrm{C}-309-\mathrm{A} 1 \\
\mathrm{C}-310-\mathrm{A} 1 \\
\mathrm{C}-311-\mathrm{A} 1 \\
\mathrm{C}-312-\mathrm{A} 1 \\
\mathrm{C}-313-\mathrm{A} 1 \\
\mathrm{C}-314-\mathrm{A} 1 \\
\mathrm{C}-315-\mathrm{A} 1 \\
\mathrm{C}-317-\mathrm{A} 1 \\
\mathrm{C}-318-\mathrm{A} 1 \\
\mathrm{C}-319-\mathrm{A} 1\end{array}$ & $\mathrm{EC}$, radial & $\begin{array}{l}1.715 \\
1.737 \\
1.729 \\
1.726 \\
1.730 \\
1.725 \\
1.711 \\
1.736 \\
1.720 \\
1.728 \\
1.735 \\
1.737 \\
1.742 \\
1.742 \\
1.746 \\
1.749 \\
1.742 \\
1.743 \\
1.743\end{array}$ & $\begin{array}{r}1609 \\
2404 \\
1548 \\
1467 \\
1406 \\
774 \\
1446 \\
1365 \\
1508 \\
1589 \\
1630 \\
2078 \\
1691 \\
1446 \\
1670 \\
1732 \\
1691 \\
1263 \\
1161\end{array}$ \\
\hline $\begin{array}{l}300-\mathrm{A} 1 \\
301-\mathrm{A} 1 \\
302-\mathrm{A} 1 \\
303-\mathrm{A} 1 \\
304-\mathrm{A} 1 \\
305-\mathrm{A} 1 \\
306-\mathrm{A} 1 \\
307-\mathrm{A} 1 \\
308-\mathrm{A} 1 \\
309-\mathrm{A} 1 \\
310-\mathrm{A} 1 \\
311-\mathrm{A} 1 \\
312-\mathrm{A} 1 \\
313-\mathrm{A} 1 \\
314-\mathrm{A} 1 \\
315-\mathrm{A} 1 \\
316-\mathrm{A} 1 \\
317-\mathrm{A} 1 \\
318-\mathrm{A} 1 \\
319-\mathrm{A} 1\end{array}$ & & $\begin{array}{l}1.752 \\
1.748 \\
1.751 \\
1.759 \\
1.755 \\
1.754 \\
1.742 \\
1.737 \\
1.727 \\
1.732 \\
1.756 \\
1.732 \\
1.749 \\
1.744 \\
1.750 \\
1.746 \\
1.744 \\
1.745 \\
1.745 \\
1.740\end{array}$ & $\begin{array}{l}1711 \\
2139 \\
1691 \\
1772 \\
1833 \\
2282 \\
1304 \\
2159 \\
2292 \\
2098 \\
2261 \\
1895 \\
1996 \\
2037 \\
1365 \\
1589 \\
1813 \\
1772 \\
2221 \\
2221\end{array}$ \\
\hline $\begin{array}{l}\text { Mean } \\
\text { Std. Dev. }\end{array}$ & 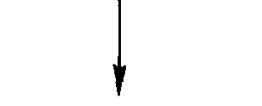 & $\begin{array}{l}\overline{1.740} \\
0.011\end{array}$ & $\begin{array}{r}1767 \\
328\end{array}$ \\
\hline
\end{tabular}


TABLE B-4 (Continued)

\begin{tabular}{|c|c|c|c|}
\hline $\begin{array}{c}\text { GA } \\
\text { Specimen } \\
\text { No. }\end{array}$ & $\begin{array}{l}\text { Location in } \\
\text { Log and } \\
\text { Orientation }\end{array}$ & $\begin{array}{l}\text { Density } \\
\left(\mathrm{g} / \mathrm{cm}^{3}\right)\end{array}$ & $\begin{array}{l}\text { U1timate } \\
\text { Strength } \\
\text { (psi) }\end{array}$ \\
\hline $\begin{array}{l}\mathrm{C}-1-\mathrm{A} 1 \\
\mathrm{C}-1-\mathrm{A} 2 \\
\mathrm{C}-2-\mathrm{A} 1 \\
\mathrm{C}-2-\mathrm{A} 2 \\
\mathrm{C}-3-\mathrm{A} 1 \\
\mathrm{C}-3-\mathrm{A} 2 \\
\mathrm{C}-4-\mathrm{A} 1 \\
\mathrm{C}-4-\mathrm{A} 2 \\
\mathrm{C}-5-\mathrm{A} 1 \\
\mathrm{C}-5-\mathrm{A} 2 \\
\mathrm{C}-6-\mathrm{A} 1 \\
\mathrm{C}-6-\mathrm{A} 2 \\
\mathrm{C}-7-\mathrm{A} 1 \\
\mathrm{C}-7-\mathrm{A} 2 \\
\mathrm{C}-8-\mathrm{A} 1 \\
\mathrm{C}-8-\mathrm{A} 2 \\
\mathrm{C}-9-\mathrm{A} 1 \\
\mathrm{C}-9-\mathrm{A} 2 \\
\mathrm{C}-10-\mathrm{A} 1 \\
\mathrm{C}-10-\mathrm{A} 2\end{array}$ & EC, axial & $\mid$ & $\begin{array}{l}1935 \\
2587 \\
1895 \\
2811 \\
2404 \\
1996 \\
2669 \\
2587 \\
2383 \\
2241 \\
2628 \\
2200 \\
2404 \\
2770 \\
2424 \\
2832 \\
1650 \\
2384 \\
2445 \\
1569\end{array}$ \\
\hline $\begin{array}{l}1-\mathrm{A} 1 \\
1-\mathrm{A} 2 \\
2-\mathrm{A} 1 \\
2-\mathrm{A} 2 \\
3-\mathrm{A} 1 \\
3-\mathrm{A} 2 \\
4-\mathrm{A} 1 \\
4-\mathrm{A} 2 \\
5-\mathrm{A} 1 \\
5-\mathrm{A} 2 \\
6-\mathrm{A} 1 \\
6-\mathrm{A} 2 \\
7-\mathrm{A} 1 \\
7-\mathrm{A} 2 \\
8-\mathrm{A} 1 \\
8-\mathrm{A} 2 \\
9-\mathrm{A} 1 \\
9-\mathrm{A} 2 \\
10-\mathrm{A} 1 \\
10-\mathrm{A} 2\end{array}$ & & $\begin{array}{l}1.754 \\
1.760 \\
1.753 \\
1.750 \\
1.742 \\
1.759 \\
1.759 \\
1.753 \\
1.756 \\
1.748 \\
1.752 \\
1.751 \\
1.762 \\
1.749 \\
1.749 \\
1.755 \\
1.754 \\
1.751 \\
1.752 \\
1.755 \\
\end{array}$ & $\begin{array}{l}2078 \\
2139 \\
2444 \\
2485 \\
2546 \\
2587 \\
1996 \\
2281 \\
1874 \\
1772 \\
2669 \\
2078 \\
1711 \\
2139 \\
2444 \\
3015 \\
2098 \\
1833 \\
2404 \\
2545 \\
\end{array}$ \\
\hline $\begin{array}{l}\text { Mean } \\
\text { Std. Dev. }\end{array}$ & 1 & $\begin{array}{l}1.744 \\
0.012\end{array}$ & $\begin{array}{r}2299 \\
348\end{array}$ \\
\hline
\end{tabular}


TABLE B-4 (Continued)

\begin{tabular}{|c|c|c|c|}
\hline $\begin{array}{c}\text { GA } \\
\text { Specimen } \\
\text { No. }\end{array}$ & $\begin{array}{l}\text { Location in } \\
\text { Log and } \\
\text { Orientation }\end{array}$ & $\begin{array}{l}\text { Density } \\
\left(\mathrm{g} / \mathrm{cm}^{3}\right)\end{array}$ & $\begin{array}{l}\text { Ultimate } \\
\text { Strength } \\
\text { (psi) }\end{array}$ \\
\hline $\begin{array}{l}\mathrm{E}-300-\mathrm{A} 1 \\
\mathrm{E}-301-\mathrm{A} 1 \\
\mathrm{E}-302-\mathrm{A} 1 \\
\mathrm{E}-303-\mathrm{A} 1 \\
\mathrm{E}-304-\mathrm{A} 1 \\
\mathrm{E}-305-\mathrm{A} 1 \\
\mathrm{E}-306-\mathrm{A} 1 \\
\mathrm{E}-307-\mathrm{A} 1 \\
\mathrm{E}-308-\mathrm{A} 1 \\
\mathrm{E}-309-\mathrm{A} 1 \\
\mathrm{E}-310-\mathrm{A} 1 \\
\mathrm{E}-311-\mathrm{A} 1 \\
\mathrm{E}-312-\mathrm{A} 1 \\
\mathrm{E}-313-\mathrm{A} 1 \\
\mathrm{E}-314-\mathrm{A} 1 \\
\mathrm{E}-315-\mathrm{A} 1 \\
\mathrm{E}-316-\mathrm{A} 1 \\
\mathrm{E}-317-\mathrm{A} 1 \\
\mathrm{E}-318-\mathrm{A} 1 \\
\mathrm{E}-319-\mathrm{A} 1 \\
\mathrm{E}-320-\mathrm{A} 1 \\
\mathrm{E}-321-\mathrm{A} 1 \\
\mathrm{E}-322-\mathrm{A} 1 \\
\mathrm{E}-323-\mathrm{A} 1\end{array}$ & EE, radial & $\begin{array}{l}1.738 \\
1.731 \\
1.718 \\
1.724 \\
1.718 \\
1.718 \\
1.733 \\
1.743 \\
1.739 \\
1.743 \\
1.745 \\
1.748 \\
1.733 \\
1.732 \\
1.724 \\
1.732 \\
1.733 \\
1.724 \\
1.737 \\
1.738 \\
1.730 \\
1.737 \\
1.723 \\
1.720\end{array}$ & $\begin{array}{l}1691 \\
1304 \\
1100 \\
1182 \\
1426 \\
1813 \\
1793 \\
1956 \\
1670 \\
2058 \\
1569 \\
1670 \\
1956 \\
1914 \\
1793 \\
1895 \\
1813 \\
1426 \\
2037 \\
2220 \\
1976 \\
2017 \\
2037 \\
1976\end{array}$ \\
\hline $\begin{array}{l}300-\mathrm{A} 1 \\
301-\mathrm{A} 1 \\
302-\mathrm{A} 1 \\
303-\mathrm{A} 1 \\
304-\mathrm{A} 1 \\
305-\mathrm{A} 1 \\
306-\mathrm{A} 1 \\
307-\mathrm{A} 1 \\
308-\mathrm{A} 1 \\
309-\mathrm{A} 1 \\
310-\mathrm{A} 1 \\
311-\mathrm{A} 1 \\
312-\mathrm{A} 1 \\
313-\mathrm{A} 1 \\
314-\mathrm{A} 1 \\
315-\mathrm{A} 1 \\
316-\mathrm{A} 1 \\
317-\mathrm{A} 1 \\
318-\mathrm{A} 1 \\
319-\mathrm{A} 1 \\
320-\mathrm{A} 1 \\
321-\mathrm{A} 1 \\
322-\mathrm{A} 1 \\
323-\mathrm{A} 1\end{array}$ & 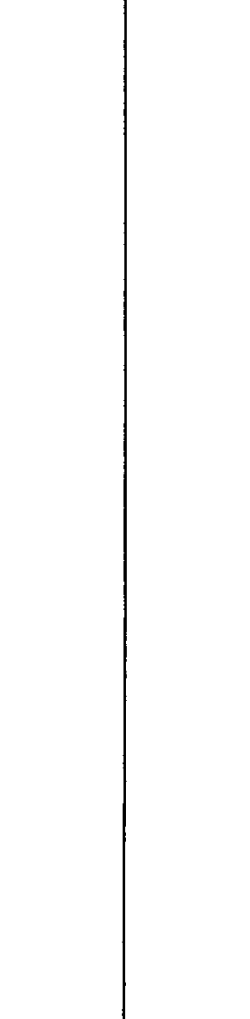 & $\begin{array}{l}1.734 \\
1.744 \\
1.742 \\
1.743 \\
1.725 \\
1.740 \\
1.751 \\
1.749 \\
1.745 \\
1.742 \\
1.746 \\
1.732 \\
1.733 \\
1.725 \\
1.725 \\
1.723 \\
1.723 \\
1.723 \\
1.732 \\
1.723 \\
-- \\
1.726 \\
1.726 \\
1.721 \\
\end{array}$ & $\begin{array}{l}1787 \\
1723 \\
1833 \\
1729 \\
2051 \\
1995 \\
1893 \\
1687 \\
2230 \\
2127 \\
2029 \\
1943 \\
2124 \\
2023 \\
2162 \\
2361 \\
2037 \\
2116 \\
2082 \\
2254 \\
-- \\
2188 \\
2283 \\
1958 \\
\end{array}$ \\
\hline $\begin{array}{l}\text { Mean } \\
\text { Std. Dev. }\end{array}$ & 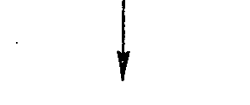 & $\begin{array}{l}1.733 \\
0.009\end{array}$ & $\begin{array}{r}1882 \\
278\end{array}$ \\
\hline
\end{tabular}


TABLE B-4 (Continued)

\begin{tabular}{|c|c|c|c|}
\hline $\begin{array}{l}\text { GA } \\
\text { Specimen } \\
\text { No. }\end{array}$ & $\begin{array}{l}\text { Location in } \\
\text { Log and } \\
\text { Orientation }\end{array}$ & $\begin{array}{l}\text { Density } \\
\left(\mathrm{g} / \mathrm{cm}^{3}\right)\end{array}$ & $\begin{array}{l}\text { Ultimate } \\
\text { Strength } \\
\text { (psi) }\end{array}$ \\
\hline $\begin{array}{l}\mathrm{E}-1-\mathrm{A} 1 \\
\mathrm{E}-2-\mathrm{A} 1 \\
\mathrm{E}-3-\mathrm{A} 1 \\
\mathrm{E}-4-\mathrm{A} 1 \\
\mathrm{E}-5-\mathrm{A} 1 \\
\mathrm{E}-6-\mathrm{A} 1 \\
\mathrm{E}-7-\mathrm{A} 1 \\
\mathrm{E}-8-\mathrm{A} 1 \\
\mathrm{E}-9-\mathrm{A} 1 \\
\mathrm{E}-10-\mathrm{A} 1 \\
\mathrm{E}-11-\mathrm{A} 1 \\
\mathrm{E}-12-\mathrm{A} 1 \\
\mathrm{E}-13-\mathrm{A} 1 \\
\mathrm{E}-14-\mathrm{A} 1 \\
\mathrm{E}-15-\mathrm{A} 1 \\
\mathrm{E}-16-\mathrm{A} 1 \\
\mathrm{E}-17-\mathrm{A} 1 \\
\mathrm{E}-18-\mathrm{A} 1 \\
\mathrm{E}-19-\mathrm{A} 1 \\
\mathrm{E}-20-\mathrm{A} 1 \\
\mathrm{E}-21-\mathrm{A} 1 \\
\mathrm{E}-22-\mathrm{A} 1 \\
\mathrm{E}-23-\mathrm{A} 1 \\
\mathrm{E}-24-\mathrm{A} 1\end{array}$ & $\mid$ & $\begin{array}{l}1.738 \\
1.725 \\
1.719 \\
1.734 \\
1.735 \\
1.728 \\
1.740 \\
1.714 \\
1.741 \\
1.741 \\
1.730 \\
1.738 \\
1.705 \\
1.731 \\
1.731 \\
1.732 \\
1.734 \\
1.746 \\
1.736 \\
1.733 \\
1.738 \\
1.723 \\
1.728 \\
1.729\end{array}$ & $\begin{array}{l}2057 \\
2404 \\
2118 \\
2444 \\
2139 \\
2261 \\
2302 \\
1874 \\
2322 \\
1996 \\
2567 \\
2689 \\
1792 \\
2628 \\
2485 \\
2485 \\
2465 \\
2180 \\
2506 \\
2302 \\
2322 \\
1833 \\
2546 \\
2546\end{array}$ \\
\hline $\begin{array}{l}1-\mathrm{A} 1 \\
2-\mathrm{A} 1 \\
3-\mathrm{A} 1 \\
4-\mathrm{A} 1 \\
5-\mathrm{A} 1 \\
6-\mathrm{A} 1 \\
7-\mathrm{A} 1 \\
8-\mathrm{A} 1 \\
9-\mathrm{A} 1 \\
10-\mathrm{A} 1 \\
11-\mathrm{A} 1 \\
12-\mathrm{A} 1 \\
13-\mathrm{A} 1 \\
14-\mathrm{A} 1 \\
15-\mathrm{A} 1 \\
16-\mathrm{A} 1 \\
17-\mathrm{A} 1 \\
18-\mathrm{A} 1 \\
19-\mathrm{A} 1 \\
20-\mathrm{A} 1 \\
21-\mathrm{A} 1 \\
22-\mathrm{A} 1 \\
23-\mathrm{A} 1 \\
24-\mathrm{A} 1\end{array}$ & 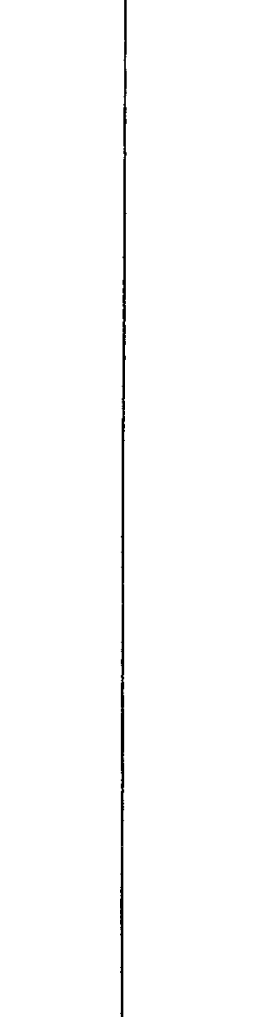 & $\begin{array}{l}1.715 \\
1.713 \\
1.725 \\
1.744 \\
1.732 \\
1.729 \\
1.728 \\
1.723 \\
1.728 \\
1.734 \\
1.739 \\
1.731 \\
1.725 \\
1.726 \\
1.731 \\
1.724 \\
1.723 \\
1.718 \\
1.730 \\
1.726 \\
1.700 \\
1.723 \\
1.732 \\
1.720 \\
\end{array}$ & $\begin{array}{l}2412 \\
2383 \\
2518 \\
2845 \\
2601 \\
2324 \\
2485 \\
2674 \\
2576 \\
2377 \\
2597 \\
2270 \\
2703 \\
2579 \\
2408 \\
2139 \\
1987 \\
2101 \\
2973 \\
2379 \\
1616 \\
2874 \\
2643 \\
2782 \\
\end{array}$ \\
\hline $\begin{array}{l}\text { Mean } \\
\text { Std. Dev. }\end{array}$ & $r$ & $\begin{array}{l}1.728 \\
0.009\end{array}$ & $\begin{array}{r}2373 \\
288\end{array}$ \\
\hline
\end{tabular}

(a) Measured at GLCC. B-13 
TABLE $B-5$

DENSITY AND TENSILE STRENGTH OF H-451, LOT 408 [Log 5651-90 (GLCC 48), whole log density $=1.75 \mathrm{~g} / \mathrm{cm}^{3}$ (a), 0.505-in.-diameter by 3.0-in.-long specimens]

\begin{tabular}{|c|c|c|c|}
\hline $\begin{array}{c}\text { GA } \\
\text { Specimen } \\
\text { No. }\end{array}$ & $\begin{array}{l}\text { Location in } \\
\text { Log and } \\
\text { Orientation }\end{array}$ & $\begin{array}{l}\text { Density } \\
\left(g / \mathrm{cm}^{3}\right)\end{array}$ & $\begin{array}{l}\text { Ultimate } \\
\text { Strength } \\
\quad \text { (psi) }\end{array}$ \\
\hline $\begin{array}{r}2 A E-500 \\
-501 \\
-502 \\
-503 \\
-504 \\
-505 \\
-506 \\
-507 \\
-508 \\
-509 \\
-510 \\
-511 \\
-512 \\
-513 \\
-514 \\
-515 \\
-516 \\
-517 \\
-518 \\
-519 \\
-520 \\
-521 \\
-522 \\
-523\end{array}$ & MLE, radial & $\begin{array}{l}1.741 \\
1.736 \\
1.738 \\
1.739 \\
1.740 \\
1.738 \\
1.737 \\
1.738 \\
1.738 \\
1.738 \\
1.740 \\
1.735 \\
1.739 \\
1.735 \\
1.737 \\
1.738 \\
1.735 \\
1.733 \\
1.730 \\
1.733 \\
1.737 \\
1.736 \\
1.737 \\
1.735\end{array}$ & $\begin{array}{l}2491 \\
2193 \\
2415 \\
2070 \\
2317 \\
2366 \\
2447 \\
2327 \\
1979 \\
2111 \\
2358 \\
2236 \\
2300 \\
1589 \\
1793 \\
1664 \\
1663 \\
1792 \\
1846 \\
1773 \\
1885 \\
1606 \\
1474 \\
1687\end{array}$ \\
\hline $\begin{array}{r}2 B E-500 \\
-501 \\
-502 \\
-503 \\
-504 \\
-505 \\
-506 \\
-507 \\
-508 \\
-509 \\
-510 \\
-511 \\
-512 \\
-513 \\
-514 \\
-515 \\
-516 \\
-517 \\
-518 \\
-519 \\
-520 \\
-521 \\
-522 \\
-523\end{array}$ & & $\begin{array}{l}1.747 \\
1.745 \\
1.744 \\
1.739 \\
1.749 \\
1.744 \\
1.748 \\
1.745 \\
1.743 \\
1.748 \\
1.749 \\
1.744 \\
1.734 \\
1.737 \\
1.736 \\
1.737 \\
1.737 \\
1.736 \\
1.732 \\
1.735 \\
1.737 \\
1.738 \\
1.730 \\
1.737\end{array}$ & $\begin{array}{l}2018 \\
1417 \\
1832 \\
1838 \\
1873 \\
2027 \\
1625 \\
1849 \\
1539 \\
1985 \\
1810 \\
1838 \\
2319 \\
2239 \\
2188 \\
2088 \\
2046 \\
2001 \\
2290 \\
2144 \\
2341 \\
2417 \\
2242 \\
2336 \\
\end{array}$ \\
\hline $\begin{array}{l}\text { Mean } \\
\text { Std. Dev. }\end{array}$ & 1 & $\begin{array}{l}1.739 \\
0.005\end{array}$ & $\begin{array}{r}2019 \\
289\end{array}$ \\
\hline
\end{tabular}


TABLE B-5 (Continued)

\begin{tabular}{|c|c|c|c|}
\hline $\begin{array}{c}\text { GA } \\
\text { Specimen } \\
\text { No. }\end{array}$ & $\begin{array}{l}\text { Location in } \\
\text { Log and } \\
\text { Orientation }\end{array}$ & $\begin{array}{l}\text { Density } \\
\left(\mathrm{g} / \mathrm{cm}^{3}\right)\end{array}$ & $\begin{array}{l}\text { U1timate } \\
\text { Strength } \\
\text { (psi) }\end{array}$ \\
\hline $\begin{array}{r}2 A E-200 \\
-201 \\
-202 \\
-203 \\
-204 \\
-205 \\
-206 \\
-207 \\
-208 \\
-209 \\
-210 \\
-211 \\
-212 \\
-213 \\
-214 \\
-215 \\
-216 \\
-217 \\
-218 \\
-219 \\
-220 \\
-221 \\
-222 \\
-223\end{array}$ & MLE, axia1 & $\begin{array}{l}1.736 \\
1.737 \\
1.739 \\
1.741 \\
1.741 \\
1.743 \\
1.742 \\
1.736 \\
1.739 \\
1.740 \\
1.740 \\
1.739 \\
1.733 \\
1.738 \\
1.741 \\
1.733 \\
1.733 \\
1.733 \\
1.740 \\
1.738 \\
1.737 \\
1.736 \\
1.732 \\
1.738\end{array}$ & $\begin{array}{l}2630 \\
2362 \\
2529 \\
2591 \\
2489 \\
2417 \\
2371 \\
2540 \\
2623 \\
2351 \\
2270 \\
2607 \\
2070 \\
2230 \\
2571 \\
2292 \\
2635 \\
2480 \\
2550 \\
2618 \\
2519 \\
2225 \\
2374 \\
2360\end{array}$ \\
\hline $\begin{array}{r}2 B E-200 \\
-201 \\
-202 \\
-203 \\
-204 \\
-205 \\
-206 \\
-207 \\
-208 \\
-209 \\
-210 \\
-211 \\
-212 \\
-213 \\
-214 \\
-215 \\
-216 \\
-217 \\
-218 \\
-219 \\
-220 \\
-221 \\
-222 \\
-223\end{array}$ & & $\begin{array}{l}1.748 \\
1.748 \\
1.743 \\
1.741 \\
1.748 \\
1.742 \\
1.741 \\
1.744 \\
1.739 \\
1.750 \\
1.745 \\
1.746 \\
1.745 \\
1.742 \\
1.745 \\
1.735 \\
1.751 \\
1.733 \\
1.735 \\
1.732 \\
1.729 \\
1.737 \\
1.729 \\
1.737 \\
\end{array}$ & $\begin{array}{l}3142 \\
3042 \\
2820 \\
3030 \\
2904 \\
2838 \\
2683 \\
2758 \\
2672 \\
2739 \\
2670 \\
2502 \\
2789 \\
2589 \\
2608 \\
2600 \\
2894 \\
2662 \\
2479 \\
2614 \\
2446 \\
1771 \\
2338 \\
2225 \\
\end{array}$ \\
\hline $\begin{array}{l}\text { Mean } \\
\text { Std. Dev. }\end{array}$ & $v$ & $\begin{array}{l}1.739 \\
0.005\end{array}$ & $\begin{array}{r}2555 \\
249\end{array}$ \\
\hline
\end{tabular}


TABLE B-5 (Continued)

\begin{tabular}{|c|c|c|c|}
\hline $\begin{array}{c}\text { GA } \\
\text { Specimen } \\
\text { No. }\end{array}$ & $\begin{array}{l}\text { Location in } \\
\text { Log and } \\
\text { Orientation }\end{array}$ & $\begin{array}{l}\text { Density } \\
\left(\mathrm{g} / \mathrm{cm}^{3}\right)\end{array}$ & $\begin{array}{l}\text { Ultimate } \\
\text { Strength } \\
\quad \text { (psi) }\end{array}$ \\
\hline $\begin{array}{r}2 A C-500 \\
-501 \\
-502 \\
-503 \\
-504 \\
-505 \\
-506 \\
-507 \\
-508 \\
-509 \\
-510 \\
-511 \\
-512 \\
-413 \\
-514 \\
-515 \\
-516 \\
-517\end{array}$ & MLC, radial & $\begin{array}{l}1.730 \\
1.733 \\
1.730 \\
1.729 \\
1.732 \\
1.733 \\
1.733 \\
1.736 \\
1.731 \\
1.732 \\
1.733 \\
1.736 \\
1.733 \\
1.733 \\
1.734 \\
1.734 \\
1.732 \\
1.734\end{array}$ & $\begin{array}{r}1731 \\
1632 \\
1912 \\
1847 \\
1848 \\
1789 \\
1621 \\
718 \\
1616 \\
1486 \\
1484 \\
1765 \\
1732 \\
1745 \\
1790 \\
1550 \\
1606 \\
1810\end{array}$ \\
\hline $\begin{array}{r}2 \mathrm{BC}-500 \\
-501 \\
-502 \\
-503 \\
-504 \\
-505 \\
-506 \\
-507 \\
-508 \\
-509 \\
-510 \\
-511 \\
-512 \\
-513 \\
-514 \\
-515 \\
-516 \\
-517\end{array}$ & & $\begin{array}{l}1.744 \\
1.738 \\
1.724 \\
1.737 \\
1.735 \\
1.738 \\
1.737 \\
1.738 \\
1.735 \\
1.729 \\
1.716 \\
1.719 \\
1.727 \\
1.723 \\
1.725 \\
1.729 \\
1.729 \\
1.727 \\
\end{array}$ & $\begin{array}{l}2108 \\
1794 \\
1658 \\
1779 \\
1694 \\
1594 \\
1946 \\
1678 \\
1660 \\
2042 \\
1936 \\
1664 \\
1755 \\
1684 \\
1426 \\
1613 \\
1343 \\
1593 \\
\end{array}$ \\
\hline $\begin{array}{l}\text { Mean } \\
\text { Std. Dev. }\end{array}$ & 1 & $\begin{array}{l}1.732 \\
0.006\end{array}$ & $\begin{array}{r}1716 \\
160\end{array}$ \\
\hline
\end{tabular}


TABLE B-5 (Continued)

\begin{tabular}{|c|c|c|c|}
\hline $\begin{array}{l}\text { GA } \\
\text { Specimen } \\
\text { No. }\end{array}$ & $\begin{array}{l}\text { Location in } \\
\text { Log and } \\
\text { Orientation }\end{array}$ & $\begin{array}{l}\text { Density } \\
\left(\mathrm{g} / \mathrm{cm}^{3}\right)\end{array}$ & $\begin{array}{l}\text { U1timate } \\
\text { Strength } \\
\text { (psi) }\end{array}$ \\
\hline $\begin{array}{r}2 A C-200 \\
-201 \\
-202 \\
-203 \\
-204 \\
-205 \\
-206 \\
-207 \\
-208 \\
-209 \\
-210 \\
-211 \\
-212 \\
-213 \\
-214 \\
-215\end{array}$ & MLC, axial & $\begin{array}{l}1.735 \\
1.730 \\
1.734 \\
1.734 \\
1.736 \\
1.733 \\
1.731 \\
1.730 \\
1.731 \\
1.730 \\
1.730 \\
1.733 \\
1.735 \\
1.733 \\
1.736 \\
1.730\end{array}$ & $\begin{array}{l}2108 \\
2181 \\
2077 \\
2073 \\
1989 \\
1961 \\
1992 \\
2348 \\
2094 \\
2116 \\
2216 \\
2196 \\
1908 \\
2235 \\
2344 \\
2284\end{array}$ \\
\hline $\begin{array}{r}2 \mathrm{BC}-200 \\
-201 \\
-202 \\
-203 \\
-204 \\
-205 \\
-206 \\
-207 \\
-208 \\
-209 \\
-210 \\
-211 \\
-212 \\
-213 \\
-214 \\
-215\end{array}$ & . & $\begin{array}{l}1.733 \\
1.741 \\
1.730 \\
1.729 \\
1.735 \\
1.739 \\
1.729 \\
1.729 \\
1.735 \\
1.739 \\
1.736 \\
1.741 \\
1.730 \\
1.728 \\
1.730 \\
1.725 \\
\end{array}$ & $\begin{array}{l}2200 \\
2170 \\
2199 \\
2095 \\
2159 \\
2172 \\
1618 \\
1911 \\
2314 \\
2226 \\
2322 \\
2193 \\
2214 \\
2185 \\
2124 \\
1865 \\
\end{array}$ \\
\hline $\begin{array}{l}\text { Mean } \\
\text { Std. Dev. }\end{array}$ & 1 & $\begin{array}{l}1.733 \\
0.004\end{array}$ & $\begin{array}{r}2129 \\
153\end{array}$ \\
\hline
\end{tabular}


TABLE B-5 (Continued)

\begin{tabular}{|c|c|c|c|}
\hline $\begin{array}{c}\text { GA } \\
\text { Specimen } \\
\text { No. }\end{array}$ & $\begin{array}{l}\text { Location in } \\
\text { Log and } \\
\text { Orientation }\end{array}$ & $\begin{array}{l}\text { Density } \\
\left(\mathrm{g} / \mathrm{cm}^{3}\right)\end{array}$ & $\begin{array}{l}\text { Ultimate } \\
\text { Strength } \\
\quad \text { (psi) }\end{array}$ \\
\hline $\begin{array}{r}1 A-E-500 \\
-501 \\
-502 \\
-503 \\
-504 \\
-505 \\
-506 \\
-507 \\
-508 \\
-509 \\
-510 \\
-511 \\
-512 \\
-513 \\
-514 \\
-515 \\
-516 \\
-517 \\
-518 \\
-519 \\
-510 \\
-521 \\
-522 \\
-523\end{array}$ & $\mathrm{EE},{ }^{\text {radial }}$ & $\begin{array}{l}1.727 \\
1.725 \\
1.721 \\
1.720 \\
1.716 \\
1.710 \\
1.701 \\
1.713 \\
1.725 \\
1.720 \\
1.727 \\
1.730 \\
1.732 \\
1.732 \\
1.731 \\
1.722 \\
1.718 \\
1.711 \\
1.736 \\
1.722 \\
1.732 \\
1.726 \\
1.726 \\
1.717\end{array}$ & $\begin{array}{l}1043 \\
1192 \\
1094 \\
1315 \\
1370 \\
1440 \\
1538 \\
1419 \\
1336 \\
931 \\
1196 \\
1310 \\
2100 \\
2200 \\
1780 \\
1908 \\
1982 \\
1651 \\
2365 \\
1678 \\
2232 \\
1684 \\
2230 \\
1251\end{array}$ \\
\hline $\begin{array}{r}1 B-E-500 \\
-501 \\
-502 \\
-503 \\
-504 \\
-505 \\
-506 \\
-507 \\
-508 \\
-509 \\
-510 \\
-511 \\
-512 \\
-513 \\
-514 \\
-515 \\
-516 \\
-517 \\
-518 \\
-519 \\
-520 \\
-521 \\
-522 \\
-523\end{array}$ & & $\begin{array}{l}1.734 \\
1.734 \\
1.733 \\
1.732 \\
1.728 \\
1.728 \\
1.739 \\
1.738 \\
1.735 \\
1.731 \\
1.332 \\
1.730 \\
1.728 \\
1.727 \\
1.728 \\
1.724 \\
1.722 \\
1.729 \\
1.729 \\
1.726 \\
1.727 \\
1.722 \\
1.718 \\
1.717 \\
\end{array}$ & $\begin{array}{l}1887 \\
1978 \\
1903 \\
1928 \\
1847 \\
1726 \\
1893 \\
1925 \\
2090 \\
2040 \\
2045 \\
1908 \\
2015 \\
2017 \\
1959 \\
2067 \\
1912 \\
1658 \\
2052 \\
2106 \\
1975 \\
2014 \\
1927 \\
1377 \\
\end{array}$ \\
\hline $\begin{array}{l}\text { Mean } \\
\text { Std. Dev. }\end{array}$ & 1 & $\begin{array}{l}1.726 \\
0.008\end{array}$ & $\begin{array}{r}1923 \\
219\end{array}$ \\
\hline
\end{tabular}


TABLE B-5 (Continued)

\begin{tabular}{|c|c|c|c|}
\hline $\begin{array}{c}\text { GA } \\
\text { Specimen } \\
\text { No. }\end{array}$ & $\begin{array}{l}\text { Location in } \\
\text { Log and } \\
\text { Orientation }\end{array}$ & $\begin{array}{l}\text { Density } \\
\left(\mathrm{g} / \mathrm{cm}^{3}\right)\end{array}$ & $\begin{array}{l}\text { Ultimate } \\
\text { Strength } \\
\text { (psi) }\end{array}$ \\
\hline $\begin{array}{r}1 \mathrm{~A}-\mathrm{E}-200 \\
-201 \\
-202 \\
-203 \\
-204 \\
-205 \\
-206 \\
-207 \\
-208 \\
-209 \\
-210 \\
-211 \\
-212 \\
-213 \\
-214 \\
-215 \\
-216 \\
-217 \\
-218 \\
-219 \\
-220 \\
-221 \\
-222 \\
-223\end{array}$ & $E E$, axial & $\begin{array}{l}1.728 \\
1.731 \\
1.734 \\
1.728 \\
1.731 \\
1.733 \\
1.732 \\
1.734 \\
1.727 \\
1.728 \\
1.733 \\
1.736 \\
1.722 \\
1.722 \\
1.718 \\
1.718 \\
1.717 \\
1.724 \\
1.726 \\
1.729 \\
1.721 \\
1.730 \\
1.726 \\
1.725\end{array}$ & $\begin{array}{l}2425 \\
2141 \\
2272 \\
2327 \\
2282 \\
2481 \\
2477 \\
2455 \\
2239 \\
2079 \\
2603 \\
2373 \\
2347 \\
2413 \\
2462 \\
2454 \\
2302 \\
2567 \\
2160 \\
2229 \\
1355 \\
1959 \\
1981 \\
1934\end{array}$ \\
\hline $\begin{array}{r}1 \mathrm{~B}-\mathrm{E}-200 \\
-201 \\
-202 \\
-203 \\
-204 \\
-205 \\
-206 \\
-207 \\
-208 \\
-209 \\
-210 \\
-212 \\
-213 \\
-214 \\
-215 \\
-216 \\
-217 \\
-218 \\
-219 \\
-220 \\
-221 \\
-222 \\
-223\end{array}$ & & $\begin{array}{l}1.734 \\
1.734 \\
1.731 \\
1.733 \\
1.729 \\
1.733 \\
1.725 \\
1.730 \\
1.728 \\
1.729 \\
1.721 \\
1.728 \\
1.724 \\
1.725 \\
1.721 \\
1.720 \\
1.720 \\
1.708 \\
1.719 \\
1.722 \\
1.716 \\
1.713 \\
1.722 \\
\end{array}$ & $\begin{array}{l}2704 \\
2787 \\
2871 \\
2394 \\
2594 \\
2623 \\
2630 \\
2611 \\
2585 \\
2520 \\
2475 \\
2461 \\
1694 \\
2369 \\
2125 \\
1781 \\
1706 \\
1667 \\
2295 \\
2284 \\
2328 \\
2073 \\
2398 \\
\end{array}$ \\
\hline $\begin{array}{l}\text { Mean } \\
\text { Std. Dev. }\end{array}$ & 1 & $\begin{array}{l}1.726 \\
0.006\end{array}$ & $\begin{array}{r}2311 \\
310\end{array}$ \\
\hline
\end{tabular}


TABLE B-5 (Continued)

\begin{tabular}{|c|c|c|c|}
\hline $\begin{array}{c}\text { GA } \\
\text { Specimen } \\
\text { No. }\end{array}$ & $\begin{array}{l}\text { Location in } \\
\text { Log and } \\
\text { Orientation }\end{array}$ & $\begin{array}{l}\text { Density } \\
\left(\mathrm{g} / \mathrm{cm}^{3}\right)\end{array}$ & $\begin{array}{l}\text { Ultimate } \\
\text { Strength } \\
\quad \text { (psi) }\end{array}$ \\
\hline $\begin{array}{r}C-500 \\
-501 \\
-502 \\
-503 \\
-504 \\
-505 \\
-506 \\
-507 \\
-508 \\
-509 \\
-510 \\
-511 \\
-512 \\
-513 \\
-514 \\
-515 \\
-516 \\
-517\end{array}$ & EC, radial & $\begin{array}{l}1.730 \\
1.729 \\
1.724 \\
1.729 \\
1.733 \\
1.726 \\
1.729 \\
1.732 \\
1.722 \\
1.735 \\
1.731 \\
1.719 \\
1.736 \\
1.730 \\
1.731 \\
1.731 \\
1.730 \\
1.721\end{array}$ & $\begin{array}{l}1532 \\
1136 \\
1368 \\
1027 \\
1476 \\
1256 \\
1206 \\
1627 \\
1630 \\
1435 \\
1197 \\
1814 \\
1249 \\
1352 \\
1671 \\
1426 \\
1835 \\
1880\end{array}$ \\
\hline $\begin{array}{r}B C-500 \\
-501 \\
-502 \\
-503 \\
-504 \\
-505 \\
-506 \\
-507 \\
-508 \\
-509 \\
-510 \\
-511 \\
-512 \\
-513 \\
-514 \\
-515 \\
-516 \\
-517\end{array}$ & & $\begin{array}{l}1.734 \\
1.733 \\
1.725 \\
1.735 \\
1.733 \\
1.730 \\
1.731 \\
1.728 \\
1.725 \\
1.731 \\
1.730 \\
1.718 \\
1.735 \\
1.732 \\
1.727 \\
1.728 \\
1.729 \\
1.726 \\
\end{array}$ & $\begin{array}{l}1875 \\
2000 \\
1716 \\
1434 \\
1404 \\
1612 \\
1502 \\
1805 \\
1272 \\
1606 \\
1926 \\
1656 \\
1363 \\
1729 \\
1734 \\
1705 \\
1815 \\
1693 \\
\end{array}$ \\
\hline $\begin{array}{l}\text { Mean } \\
\text { Std. Dev. }\end{array}$ & 1 & $\begin{array}{l}1.729 \\
0.004\end{array}$ & $\begin{array}{r}1553 \\
245\end{array}$ \\
\hline
\end{tabular}


TABLE B-5 (Continued)

\begin{tabular}{|c|c|c|c|}
\hline $\begin{array}{c}\text { GA } \\
\text { Specimen } \\
\text { No. }\end{array}$ & $\begin{array}{l}\text { Location in } \\
\text { Log and } \\
\text { Orientation }\end{array}$ & $\begin{array}{l}\text { Density } \\
\left(\mathrm{g} / \mathrm{cm}^{3}\right)\end{array}$ & $\begin{array}{l}\text { U1timate } \\
\text { Strength } \\
\text { (psi) }\end{array}$ \\
\hline $\begin{array}{l}C-200 \\
-201 \\
-202 \\
-203 \\
-204 \\
-205 \\
-206 \\
-208 \\
-209 \\
-210 \\
-211 \\
-212 \\
-213 \\
-214 \\
-215\end{array}$ & EC, axial & $\begin{array}{l}1.718 \\
1.728 \\
1.731 \\
1.731 \\
1.722 \\
1.727 \\
1.724 \\
1.718 \\
1.725 \\
1.724 \\
1.727 \\
1.733 \\
1.732 \\
1.732 \\
1.730\end{array}$ & $\begin{array}{l}2594 \\
2487 \\
2191 \\
2280 \\
2141 \\
2030 \\
2403 \\
1861 \\
2026 \\
2058 \\
1958 \\
2054 \\
2278 \\
2098 \\
2113\end{array}$ \\
\hline $\begin{array}{r}1 \mathrm{BC}-200 \\
-201 \\
-202 \\
-203 \\
-204 \\
-205 \\
-206 \\
-207 \\
-208 \\
-209 \\
-210 \\
-211 \\
-212 \\
-213 \\
-214 \\
-215\end{array}$ & & $\begin{array}{l}1.732 \\
1.731 \\
1.737 \\
1.728 \\
1.719 \\
1.729 \\
1.731 \\
1.734 \\
1.737 \\
1.728 \\
1.732 \\
1.728 \\
1.729 \\
1.728 \\
1.726 \\
1.730 \\
\end{array}$ & $\begin{array}{l}1980 \\
2271 \\
2455 \\
2440 \\
1907 \\
2323 \\
2422 \\
2514 \\
2358 \\
2158 \\
2138 \\
2436 \\
1330 \\
2535 \\
2328 \\
2361 \\
\end{array}$ \\
\hline $\begin{array}{l}\text { Mean } \\
\text { Std. Dev. }\end{array}$ & † & $\begin{array}{l}1.728 \\
0.005\end{array}$ & $\begin{array}{r}2212 \\
254\end{array}$ \\
\hline
\end{tabular}

(a) Measured at GLCC. 
TABLE B-6

DENSITY AND TENSILE PROPERTIES OF H-451, LOT 408

DENSITY AND TENSILE PROPERTIES OF H-451, LOT 408
[Log $5651-86$ (GLCC 52), whole log density $=1.74 \mathrm{~g} / \mathrm{cm}^{3}{ }^{(a)}$,
0.505 -in.-diameter by 3.0 -in.-long specimens]

\begin{tabular}{|c|c|c|c|c|c|c|}
\hline $\begin{array}{c}\text { GA } \\
\text { Specimen } \\
\text { No. }\end{array}$ & $\begin{array}{l}\text { Location in } \\
\text { Log and } \\
\text { Orientation }\end{array}$ & $\begin{array}{l}\text { Density } \\
\left(\mathrm{g} / \mathrm{cm}^{3}\right)\end{array}$ & $\mid \begin{array}{l}\text { Modulus of } \\
\text { Elasticity } \\
\mathrm{x} 10^{-6(b)} \\
\quad(\mathrm{psi})\end{array}$ & $\begin{array}{c}\text { Permanent Set } \\
\text { After First } \\
\text { Loading } \\
(\%) \\
\end{array}$ & \begin{tabular}{|c|} 
Strain at \\
Fracture \\
$(\%)$
\end{tabular} & $\begin{array}{c}\text { U1timate } \\
\text { Strength } \\
\text { (psi) }\end{array}$ \\
\hline $\begin{array}{r}5651-86-3 \mathrm{~A}-\mathrm{L} 002 \mathrm{~A} \\
-\mathrm{L} 002 \mathrm{~B} \\
-\mathrm{L} 003 \mathrm{~A} \\
-\mathrm{L} 003 \mathrm{~B} \\
-3 \mathrm{~B}-\mathrm{L} 002 \mathrm{~A} \\
-\mathrm{L} 002 \mathrm{~B}\end{array}$ & MLC, axial & $\begin{array}{l}1.702 \\
1.710 \\
1.711 \\
1.703 \\
1.699 \\
1.686 \\
\end{array}$ & $\begin{array}{l}1.13 \\
1.14 \\
1.25 \\
1.23 \\
0.97 \\
0.93 \\
\end{array}$ & $\begin{array}{l}0.010 \\
0.010 \\
0.008 \\
0.010 \\
0.022 \\
0.014 \\
\end{array}$ & $\begin{array}{l}0.250 \\
0.275 \\
0.245 \\
0.225 \\
0.268 \\
0.265 \\
\end{array}$ & $\begin{array}{l}1965 \\
2163 \\
2129 \\
2009 \\
2014 \\
1984 \\
\end{array}$ \\
\hline $\begin{array}{l}\text { Mean } \\
\text { Std. Dev. }\end{array}$ & 1 & $\begin{array}{l}1.702 \\
0.009\end{array}$ & $\begin{array}{l}1.11 \\
0.132\end{array}$ & $\begin{array}{l}0.012 \\
0.005\end{array}$ & $\begin{array}{l}0.254 \\
0.018\end{array}$ & $\begin{array}{r}2044 \\
82\end{array}$ \\
\hline $\begin{array}{r}5651-86-3 A-L 005 \\
-L 006 \\
-L 007 \\
-L 008 B \\
-3 B-L 005 \\
-L 007\end{array}$ & MLC, radial & $\begin{array}{l}1.712 \\
1.707 \\
1.707 \\
1.698 \\
1.702 \\
1.700 \\
\end{array}$ & $\begin{array}{l}1.07 \\
1.06 \\
1.02 \\
1.06 \\
0.99 \\
0.94 \\
\end{array}$ & $\begin{array}{l}0.013 \\
0.013 \\
0.012 \\
0.013 \\
0.018 \\
0.022 \\
\end{array}$ & $\begin{array}{l}0.204 \\
0.255 \\
0.145 \\
0.205 \\
0.215 \\
0.210 \\
\end{array}$ & $\begin{array}{l}1648 \\
1862 \\
1135 \\
1623 \\
1551 \\
1488 \\
\end{array}$ \\
\hline $\begin{array}{l}\text { Mean } \\
\text { Std. Dev. }\end{array}$ & 1 & $\begin{array}{l}1.704 \\
0.005\end{array}$ & $\begin{array}{l}1.02 \\
0.051\end{array}$ & $\begin{array}{l}0.015 \\
0.004\end{array}$ & $\begin{array}{l}0.206 \\
0.035\end{array}$ & $\begin{array}{r}1551 \\
240\end{array}$ \\
\hline $\begin{array}{r}5651-86-3 A-L 009 A \\
-L 009 B \\
-L 010 A \\
-L 010 B \\
-3 B-L 009 A \\
-L 009 B \\
-L 012\end{array}$ & MLE, axia1 & $\begin{array}{l}1.720 \\
1.712 \\
1.720 \\
1.723 \\
1.714 \\
1.713 \\
1.718 \\
\end{array}$ & $\begin{array}{l}1.32 \\
1.32 \\
1.38 \\
1.36 \\
1.18 \\
1.17 \\
1.34 \\
\end{array}$ & $\begin{array}{l}0.011 \\
0.011 \\
0.007 \\
0.000 \\
0.017 \\
0.018 \\
0.020 \\
\end{array}$ & $\begin{array}{l}0.315 \\
0.315 \\
0.301 \\
0.335 \\
0.310 \\
(c) \\
0.310 \\
\end{array}$ & $\begin{array}{l}2857 \\
2774 \\
2789 \\
3038 \\
2486 \\
(\mathrm{c}) \\
2646 \\
\end{array}$ \\
\hline $\begin{array}{l}\text { Mean } \\
\text { Std. Dev. }\end{array}$ & 1 & $\begin{array}{l}1.717 \\
0.004\end{array}$ & $\begin{array}{l}1.30 \\
0.085\end{array}$ & $\begin{array}{l}0.012 \\
0.007\end{array}$ & $\begin{array}{l}0.314 \\
0.011\end{array}$ & $\begin{array}{r}1765 \\
187\end{array}$ \\
\hline $\begin{array}{r}5651-86-3 \mathrm{~A}-\mathrm{L} 013 \\
-\mathrm{L} 014 \\
-\mathrm{L} 015 \\
-3 \mathrm{~B}-\mathrm{L} 013 \\
-\mathrm{L} 014 \\
-\mathrm{L} 015\end{array}$ & MLE, radial & $\begin{array}{l}1.712 \\
1.718 \\
1.716 \\
1.715 \\
1.713 \\
1.720 \\
\end{array}$ & $\begin{array}{l}1.10 \\
1.09 \\
0.94 \\
1.00 \\
0.97 \\
0.96 \\
\end{array}$ & $\begin{array}{l}0.012 \\
0.010 \\
0.006 \\
0.018 \\
0.022 \\
0.025 \\
\end{array}$ & $\begin{array}{l}0.255 \\
0.220 \\
0.195 \\
0.285 \\
0.285 \\
0.245 \\
\end{array}$ & $\begin{array}{l}1956 \\
1882 \\
1629 \\
1961 \\
1893 \\
1724 \\
\end{array}$ \\
\hline $\begin{array}{l}\text { Mean } \\
\text { Std. Dev. }\end{array}$ & 1 & $\begin{array}{l}1.716 \\
0.003\end{array}$ & $\begin{array}{l}1.01 \\
0.069\end{array}$ & $\begin{array}{l}0.015 \\
0.007\end{array}$ & $\begin{array}{l}0.247 \\
0.036\end{array}$ & $\begin{array}{r}1841 \\
135\end{array}$ \\
\hline
\end{tabular}

(a) Measured at GLCC.

(b) Specimens were loaded to $1000 \mathrm{psi}$, unloaded to zero stress and reloaded to failure while recording the stress-strain curve. Modulus of elasticity = chord modulus between 100 and 1000 psi on second loading.

(c) Bond failure. 
TABLE B-7

DENSITY AND TENSILE PROPERTIES OF H-451, LOT 408

[Log 5651-86 (GLCC 52), whole $10 \mathrm{~g}$ density $=1.74 \mathrm{~g} / \mathrm{cm}^{3}{ }^{(a)}$, 0.25 -in.-diameter by 0.90 -in.-long specimens]

\begin{tabular}{|c|c|c|c|c|c|c|}
\hline $\begin{array}{c}\text { GA } \\
\text { Specimen } \\
\text { No. }\end{array}$ & $\begin{array}{c}\text { Location in } \\
\text { Log and } \\
\text { Orientation }\end{array}$ & $\begin{array}{l}\text { Density } \\
\left(\mathrm{g} / \mathrm{cm}^{3}\right)\end{array}$ & $\mid \begin{array}{c}\text { Modulus of } \\
\text { Elasticity } \\
x \quad 10^{-6(b)} \\
(p s i)\end{array}$ & $\begin{array}{c}\text { Permanent Set } \\
\text { After First } \\
\text { Loading } \\
(\%)\end{array}$ & $\begin{array}{c}\text { Strain at } \\
\text { Fracture } \\
(\%)\end{array}$ & $\begin{array}{l}\text { Ultimate } \\
\text { Strength } \\
\text { (psi) }\end{array}$ \\
\hline $\begin{array}{r}5651-86-3 \mathrm{~A}-006 \mathrm{~B} \\
-007 \mathrm{~B} \\
-012 \mathrm{~B} \\
-013 \mathrm{~B} \\
-018 \mathrm{~B} \\
-019 \mathrm{~B} \\
-024 \mathrm{~B} \\
-025 \mathrm{~B} \\
-027 \mathrm{~B} \\
-028 \mathrm{~B} \\
3 \mathrm{~B}-006 \mathrm{~B} \\
-007 \mathrm{~B} \\
-012 \mathrm{~B} \\
-013 \mathrm{~B} \\
-018 \mathrm{~B} \\
-019 \mathrm{~B} \\
-024 \mathrm{~B} \\
-025 \mathrm{~B} \\
-027 \mathrm{~B} \\
-028 \mathrm{~B}\end{array}$ & MLC, axial & $\begin{array}{l}1.700 \\
1.686 \\
1.715 \\
1.700 \\
1.702 \\
1.707 \\
1.701 \\
1.693 \\
1.701 \\
1.690 \\
1.685 \\
1.701 \\
1.700 \\
1.691 \\
1.701 \\
1.688 \\
1.706 \\
1.710 \\
1.695 \\
1.686 \\
\end{array}$ & $\begin{array}{l}1.06 \\
1.125 \\
1.125 \\
(\mathrm{c}) \\
0.86 \\
1.06 \\
0.90 \\
1.06 \\
1.06 \\
1.00 \\
1.15 \\
1.29 \\
1.125 \\
1.125 \\
0.98 \\
1.20 \\
1.125 \\
1.20 \\
1.125 \\
1.00 \\
\end{array}$ & $\begin{array}{l}0.020 \\
0.020 \\
0.035 \\
(\mathrm{c}) \\
0.030 \\
0.025 \\
0.030 \\
0.020 \\
0.015 \\
0.026 \\
0.025 \\
0.027 \\
0.030 \\
0.030 \\
0.030 \\
0.025 \\
0.017 \\
0.020 \\
0.010 \\
0.020 \\
\end{array}$ & $\begin{array}{l}0.280 \\
0.260 \\
0.325 \\
0.360 \\
0.295 \\
0.325 \\
0.325 \\
0.185 \\
0.375 \\
0.190 \\
0.210 \\
0.300 \\
0.370 \\
0.250 \\
0.265 \\
0.285 \\
0.295 \\
0.255 \\
0.280 \\
0.250 \\
\end{array}$ & $\begin{array}{l}2095 \\
1993 \\
2199 \\
1789 \\
1710 \\
2192 \\
2075 \\
1486 \\
2153 \\
1564 \\
1587 \\
2034 \\
1931 \\
1671 \\
1626 \\
1585 \\
2138 \\
2092 \\
1730 \\
1787 \\
\end{array}$ \\
\hline $\begin{array}{l}\text { Mean } \\
\text { Std. Dev. }\end{array}$ & 9 & $\begin{array}{l}1.698 \\
0.008\end{array}$ & $\begin{array}{l}1.08 \\
0.104\end{array}$ & $\begin{array}{l}0.024 \\
0.006\end{array}$ & $\begin{array}{l}0.279 \\
0.047\end{array}$ & $\begin{array}{r}1872 \\
242\end{array}$ \\
\hline $\begin{array}{r}-86-3 \mathrm{~A}-036 \mathrm{~B} \\
-037 \mathrm{~B} \\
-042 \mathrm{~B} \\
-043 \mathrm{~B} \\
-048 \mathrm{~B} \\
-049 \mathrm{~B} \\
-054 \mathrm{~B} \\
-055 \mathrm{~B} \\
-060 \mathrm{~A} \\
-060 \mathrm{~B} \\
-3 \mathrm{~B}-036 \mathrm{~B} \\
-037 \mathrm{~B} \\
-042 \mathrm{~B} \\
-043 \mathrm{~B} \\
-048 \mathrm{~B} \\
-049 \mathrm{~B} \\
-054 \mathrm{~B} \\
-055 \mathrm{~B} \\
-060 \mathrm{~A} \\
-060 \mathrm{~B}\end{array}$ & MLC, radial & $\begin{array}{l}1.687 \\
1.680 \\
1.700 \\
1.697 \\
1.693 \\
1.694 \\
1.692 \\
1.695 \\
1.698 \\
1.700 \\
1.692 \\
1.679 \\
1.715 \\
1.699 \\
1.697 \\
1.701 \\
1.710 \\
1.692 \\
1.696 \\
1.687 \\
\end{array}$ & $\begin{array}{l}1.06 \\
1.02 \\
1.10 \\
1.10 \\
0.95 \\
1.00 \\
0.95 \\
0.90 \\
1.00 \\
1.20 \\
1.125 \\
1.125 \\
0.86 \\
0.82 \\
1.29 \\
1.06 \\
1.125 \\
1.00 \\
1.125 \\
1.06 \\
\end{array}$ & $\begin{array}{l}0.030 \\
0.027 \\
0.020 \\
0.025 \\
0.020 \\
0.015 \\
0.025 \\
0.035 \\
0.020 \\
0.025 \\
0.025 \\
0.025 \\
0.040 \\
0.040 \\
0.020 \\
0.030 \\
0.020 \\
0.035 \\
0.030 \\
0.020 \\
\end{array}$ & $\begin{array}{l}0.255 \\
0.295 \\
0.280 \\
0.275 \\
0.225 \\
0.230 \\
0.305 \\
0.320 \\
0.205 \\
0.175 \\
0.225 \\
0.295 \\
0.350 \\
0.265 \\
0.285 \\
0.200 \\
0.225 \\
0.245 \\
0.240 \\
0.285 \\
\end{array}$ & $\begin{array}{l}1730 \\
1889 \\
2034 \\
1953 \\
1588 \\
1711 \\
1909 \\
2029 \\
1671 \\
1361 \\
1564 \\
1603 \\
1915 \\
2015 \\
2162 \\
1858 \\
1565 \\
1221 \\
1810 \\
1848 \\
\end{array}$ \\
\hline $\begin{array}{l}\text { Mean } \\
\text { Std. Dev. }\end{array}$ & $r$ & $\begin{array}{l}1.695 \\
0.008\end{array}$ & $\begin{array}{l}1.04 \\
0.114\end{array}$ & $\begin{array}{l}0.026 \\
0.007\end{array}$ & $\begin{array}{l}0.259 \\
0.044\end{array}$ & $\begin{array}{r}1772 \\
239\end{array}$ \\
\hline
\end{tabular}


TABLE B-7 (Continued)

\begin{tabular}{|c|c|c|c|c|c|c|}
\hline $\begin{array}{c}\text { GA } \\
\text { Specimen } \\
\text { No. }\end{array}$ & $\begin{array}{l}\text { Location in } \\
\text { Log and } \\
\text { Orientation }\end{array}$ & $\begin{array}{l}\text { Density } \\
\left(\mathrm{g} / \mathrm{cm}^{3}\right)\end{array}$ & $\begin{array}{l}\text { Modulus of } \\
\text { Elasticity } \\
\mathrm{x} 10^{-6}(\mathrm{~b}) \\
\text { (psi) }\end{array}$ & $\begin{array}{c}\text { Permanent Set } \\
\text { After First } \\
\text { Loading } \\
(\%)\end{array}$ & $\begin{array}{c}\text { Strain at } \\
\text { Fracture } \\
(\%)\end{array}$ & $\begin{array}{l}\text { Ultimate } \\
\text { Strength } \\
\text { (psi) }\end{array}$ \\
\hline $\begin{array}{r}5651-86-3 \mathrm{~A}-106 \mathrm{~B} \\
-107 \mathrm{~B} \\
-112 \mathrm{~B} \\
-113 \mathrm{~B} \\
-118 \mathrm{~B} \\
-119 \mathrm{~B} \\
-124 \mathrm{~B} \\
-125 \mathrm{~B} \\
-127 \mathrm{~B} \\
-128 \mathrm{~B} \\
3 \mathrm{~B}-106 \mathrm{~B} \\
-107 \mathrm{~B} \\
-112 \mathrm{~B} \\
-113 \mathrm{~B} \\
-118 \mathrm{~B} \\
-119 \mathrm{~B} \\
-124 \mathrm{~B} \\
-125 \mathrm{~B} \\
-127 \mathrm{~B} \\
-128 \mathrm{~B}\end{array}$ & MLE, axial & $\begin{array}{l}1.705 \\
1.706 \\
1.698 \\
1.706 \\
1.707 \\
1.707 \\
1.713 \\
1.711 \\
1.692 \\
1.714 \\
1.715 \\
1.706 \\
1.710 \\
1.715 \\
1.704 \\
1.699 \\
1.703 \\
1.709 \\
1.696 \\
1.704 \\
\end{array}$ & $\begin{array}{l}1.38 \\
1.29 \\
1.20 \\
1.15 \\
1.20 \\
1.29 \\
1.00 \\
1.125 \\
1.125 \\
1.125 \\
1.20 \\
1.20 \\
1.20 \\
1.125 \\
1.29 \\
1.50 \\
1.20 \\
1.20 \\
1.29 \\
1.20 \\
\end{array}$ & $\begin{array}{l}0.030 \\
0.030 \\
0.020 \\
0.030 \\
0.017 \\
0.021 \\
0.025 \\
0.040 \\
0.030 \\
0.035 \\
0.007 \\
0.015 \\
0.020 \\
0.025 \\
0.020 \\
0.020 \\
0.035 \\
0.025 \\
0.025 \\
0.027 \\
\end{array}$ & $\begin{array}{l}0.255 \\
0.375 \\
0.365 \\
0.300 \\
0.305 \\
0.320 \\
0.325 \\
0.370 \\
0.315 \\
0.335 \\
0.285 \\
0.350 \\
0.310 \\
0.335 \\
0.240 \\
0.320 \\
0.400 \\
0.360 \\
0.350 \\
0.240 \\
\end{array}$ & $\begin{array}{l}2215 \\
2825 \\
2647 \\
2339 \\
2541 \\
2545 \\
2461 \\
2604 \\
2276 \\
2524 \\
2520 \\
2805 \\
2504 \\
2524 \\
2117 \\
2620 \\
2746 \\
2782 \\
2785 \\
2114\end{array}$ \\
\hline $\begin{array}{l}\text { Mean } \\
\text { Std. Dev. }\end{array}$ & 1 & $\begin{array}{l}1.706 \\
0.006\end{array}$ & $\begin{array}{l}1.21 \\
0.106\end{array}$ & $\begin{array}{l}0.025 \\
0.008\end{array}$ & $\begin{array}{l}0.323 \\
0.044\end{array}$ & $\begin{array}{r}2525 \\
219\end{array}$ \\
\hline $\begin{array}{r}5651-86-3 \mathrm{~A}-136 \mathrm{~B} \\
-137 \mathrm{~B} \\
-142 \mathrm{~B} \\
-143 \mathrm{~B} \\
-148 \mathrm{~B} \\
-149 \mathrm{~B} \\
-154 \mathrm{~B} \\
-155 \mathrm{~B} \\
-160 \mathrm{~A} \\
-160 \mathrm{~B} \\
3 \mathrm{~B}-136 \mathrm{~B} \\
-137 \mathrm{~B} \\
-142 \mathrm{~B} \\
-143 \mathrm{~B} \\
-148 \mathrm{~B} \\
-149 \mathrm{~B} \\
-154 \mathrm{~B} \\
-155 \mathrm{~B} \\
-160 \mathrm{~A} \\
-160 \mathrm{~B}\end{array}$ & MLE, radial & $\begin{array}{l}1.717 \\
1.714 \\
1.714 \\
1.704 \\
1.715 \\
1.710 \\
1.720 \\
1.714 \\
1.716 \\
1.713 \\
1.714 \\
1.714 \\
1.705 \\
1.703 \\
1.706 \\
1.703 \\
1.710 \\
1.709 \\
1.713 \\
1.713 \\
\end{array}$ & $\begin{array}{l}1.125 \\
1.06 \\
1.08 \\
1.00 \\
1.00 \\
1.08 \\
0.82 \\
1.06 \\
0.86 \\
1.06 \\
1.00 \\
1.00 \\
1.125 \\
1.125 \\
0.82 \\
0.86 \\
1.06 \\
1.06 \\
1.125 \\
1.20 \\
\end{array}$ & $\begin{array}{l}0.030 \\
0.035 \\
0.050 \\
0.020 \\
0.037 \\
0.030 \\
0.025 \\
0.030 \\
0.025 \\
0.032 \\
0.025 \\
0.030 \\
0.030 \\
0.020 \\
0.040 \\
0.025 \\
0.015 \\
0.030 \\
0.020 \\
0.015 \\
\end{array}$ & $\begin{array}{l}0.335 \\
0.345 \\
0.395 \\
0.245 \\
0.310 \\
0.305 \\
0.295 \\
0.260 \\
0.295 \\
0.295 \\
0.335 \\
0.370 \\
0.320 \\
0.240 \\
0.450 \\
0.345 \\
0.300 \\
0.340 \\
0.260 \\
0.340\end{array}$ & $\begin{array}{l}2271 \\
2256 \\
2343 \\
1648 \\
2073 \\
2132 \\
1560 \\
1865 \\
1732 \\
2138 \\
2313 \\
2099 \\
2439 \\
2012 \\
2285 \\
2040 \\
2339 \\
2234 \\
2091 \\
2293\end{array}$ \\
\hline $\begin{array}{l}\text { Mean } \\
\text { Std. Dev. }\end{array}$ & 1 & $\begin{array}{l}1.711 \\
0.005\end{array}$ & $\begin{array}{l}1.03 \\
0.108\end{array}$ & $\begin{array}{l}0.028 \\
0.008\end{array}$ & $\begin{array}{l}0.319 \\
0.051\end{array}$ & $\begin{array}{r}2108 \\
243\end{array}$ \\
\hline
\end{tabular}

(a) Measured at GLCC.

(b) Specimens were loaded to $1000 \mathrm{psi}$, unloaded to zero stress and reloaded to failure while recording the stress-strain curve. Modulus of elasticity $=$ chord modulus between 100 and 1000 psi on second loading.

(c) Specimen not cycled. 
TABLE B-8

DENSITY AND TENSILE PROPERTIES OF H-451, LOT 426

$\left[\log 6484-33\right.$ (GLCC 92), whole $10 \mathrm{~g}$ density $=1.75 \mathrm{~g} / \mathrm{cm}^{3}$ (a), 0.505-in.-diameter by 3.00-in.-long specimens]

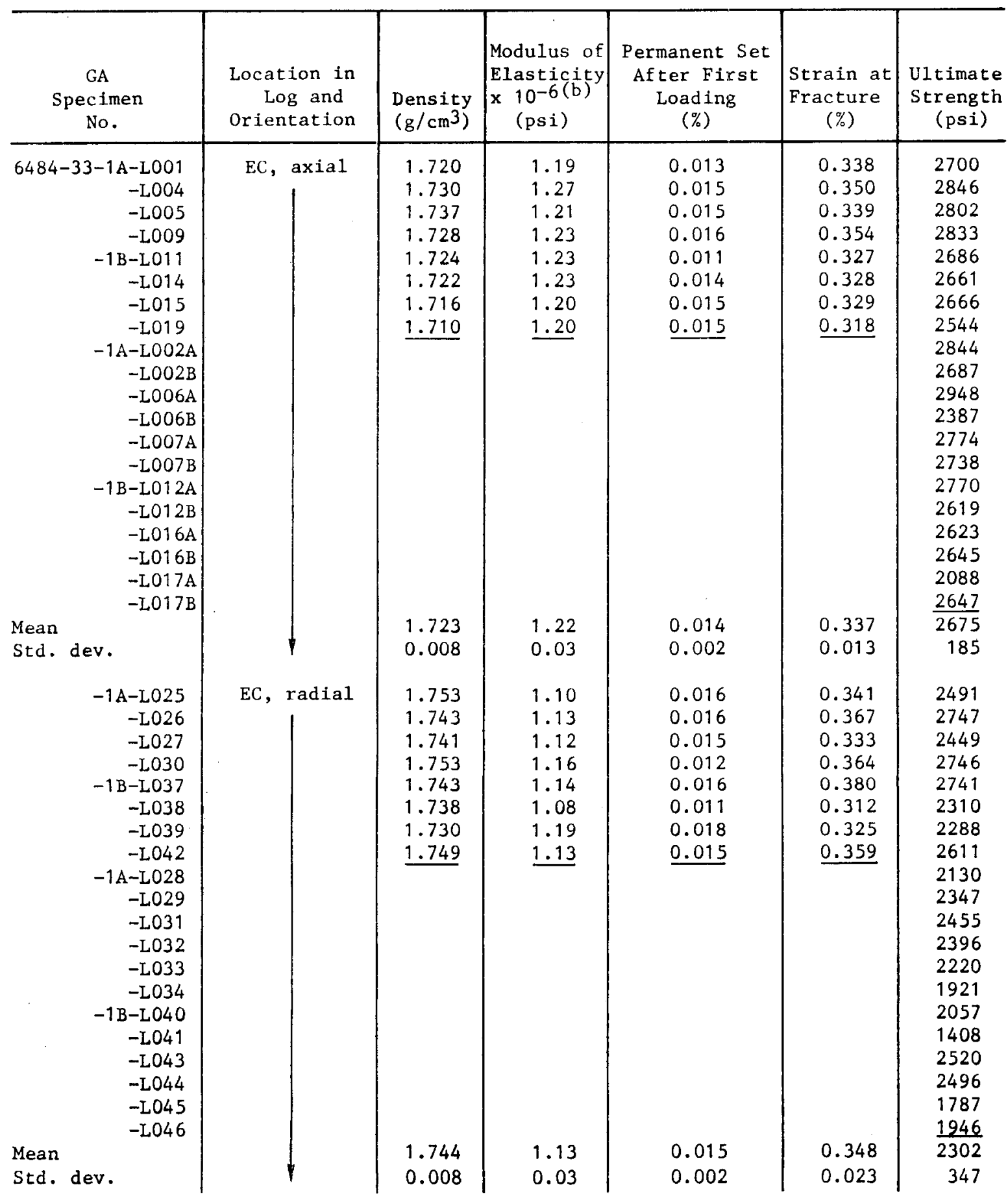


TABLE B-8 (Continued)

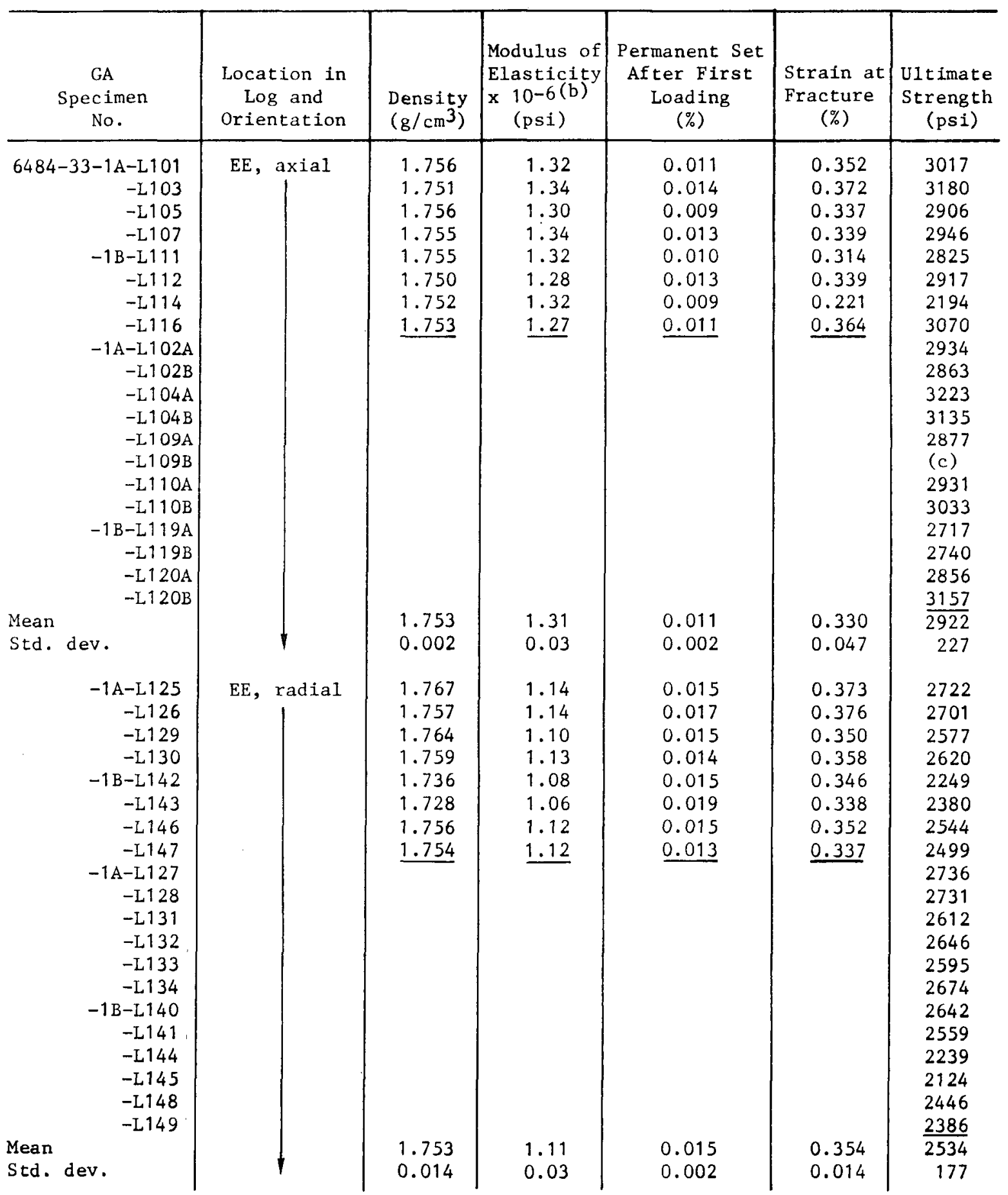


TABLE B-8 (Continued)

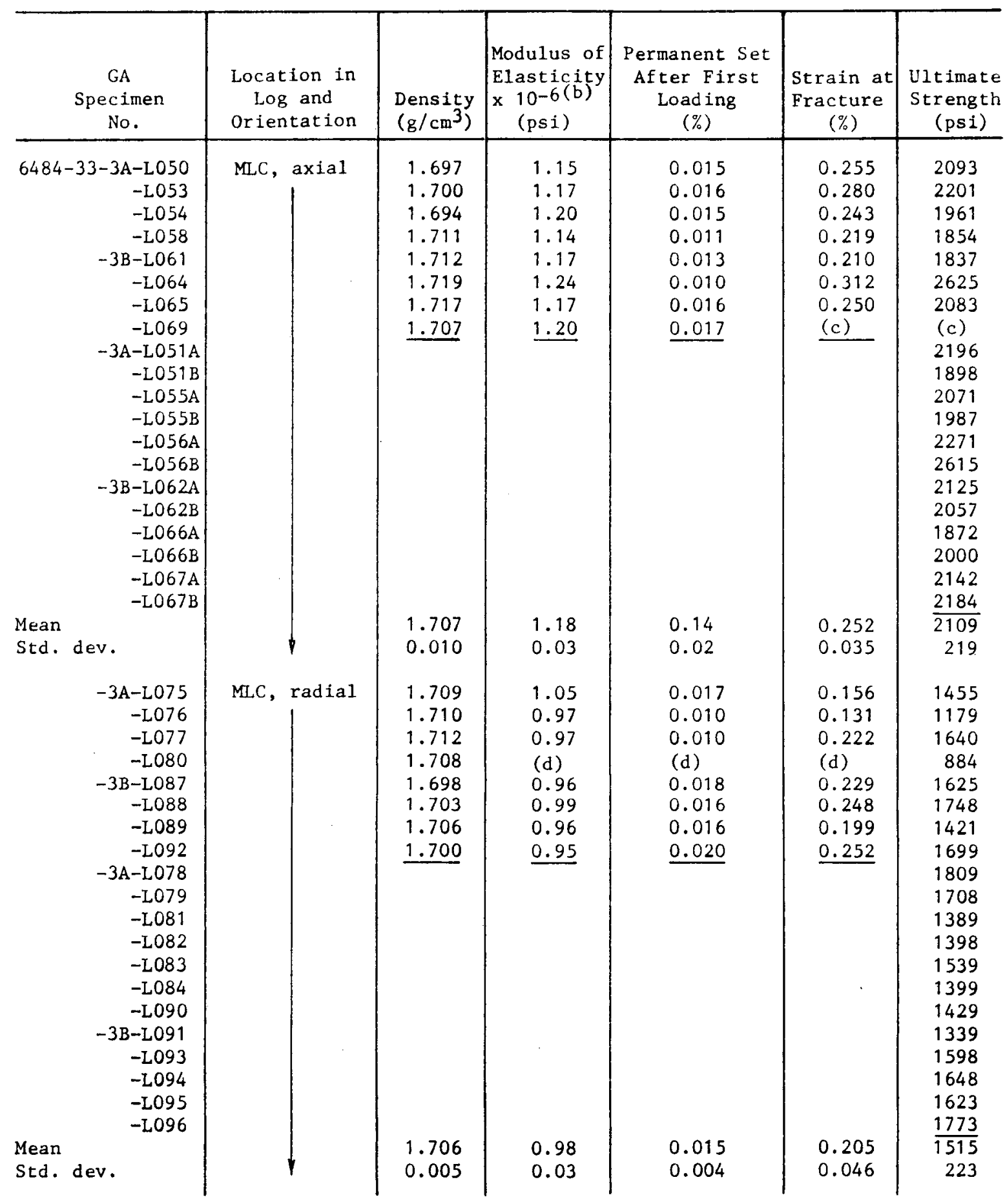


TABLE B-8 (Continued)

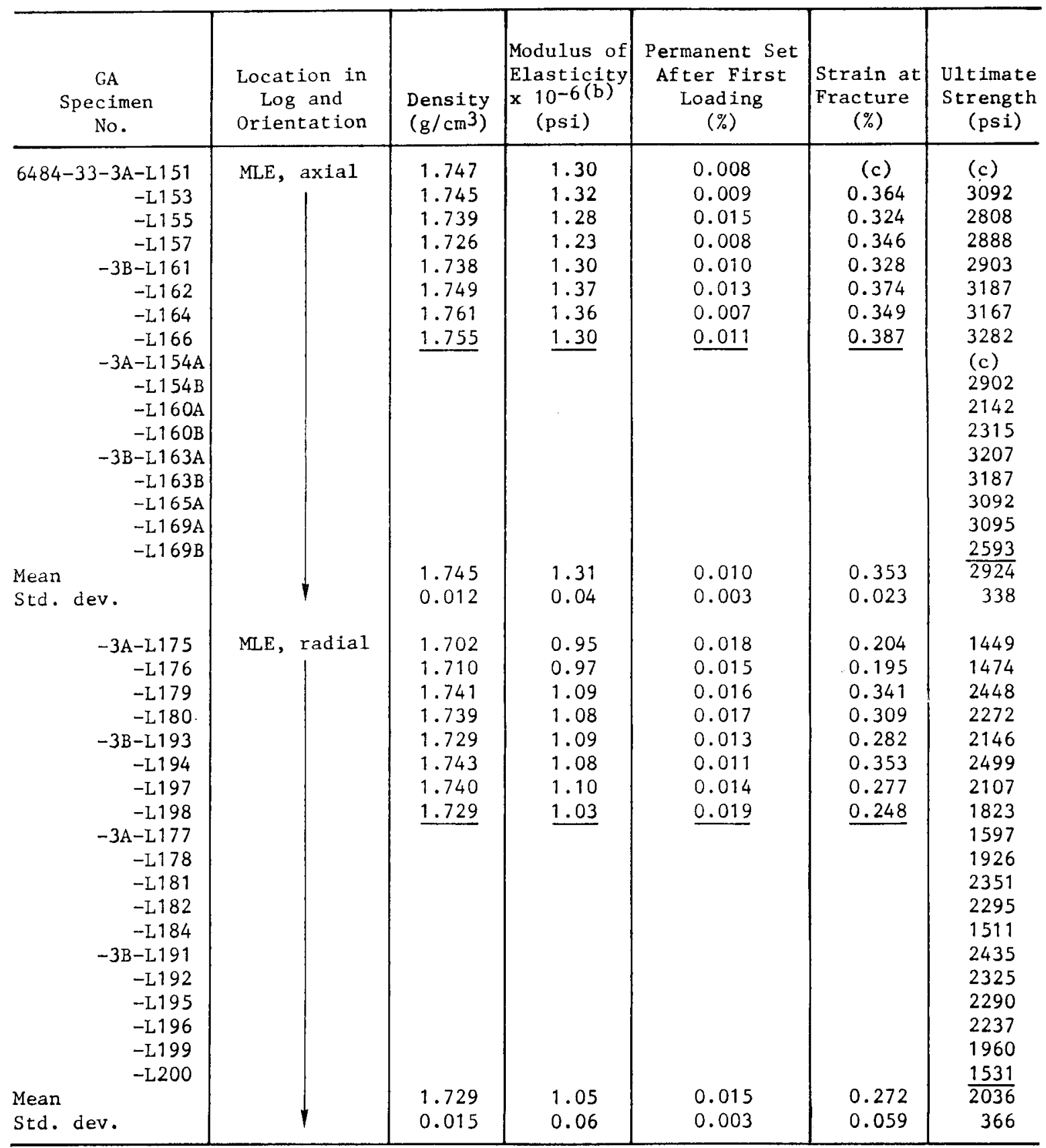

(a) Measured at GLCC.

(b) Specimens were loaded to $1000 \mathrm{psi}$, unloaded to zero stress, and reloaded to failure while recording the stress-strain curve. Modulus of elasticity $=$ chord modulus between 100 and $1000 \mathrm{psi}$ on second loading.

(c) Bond failure.

(d) Specimen broke during first loading cycle. 
TABLE B-9

DENSITY AND TENSILE PROPERTIES OF H-451, LOT 426

[ Log 6484-34 (GLCC 198), whole $\log$ density $=1.73 \mathrm{~g} / \mathrm{cm}^{3}$ (a), 0.505-in.-diameter by 3.00-in.-1ong specimens]

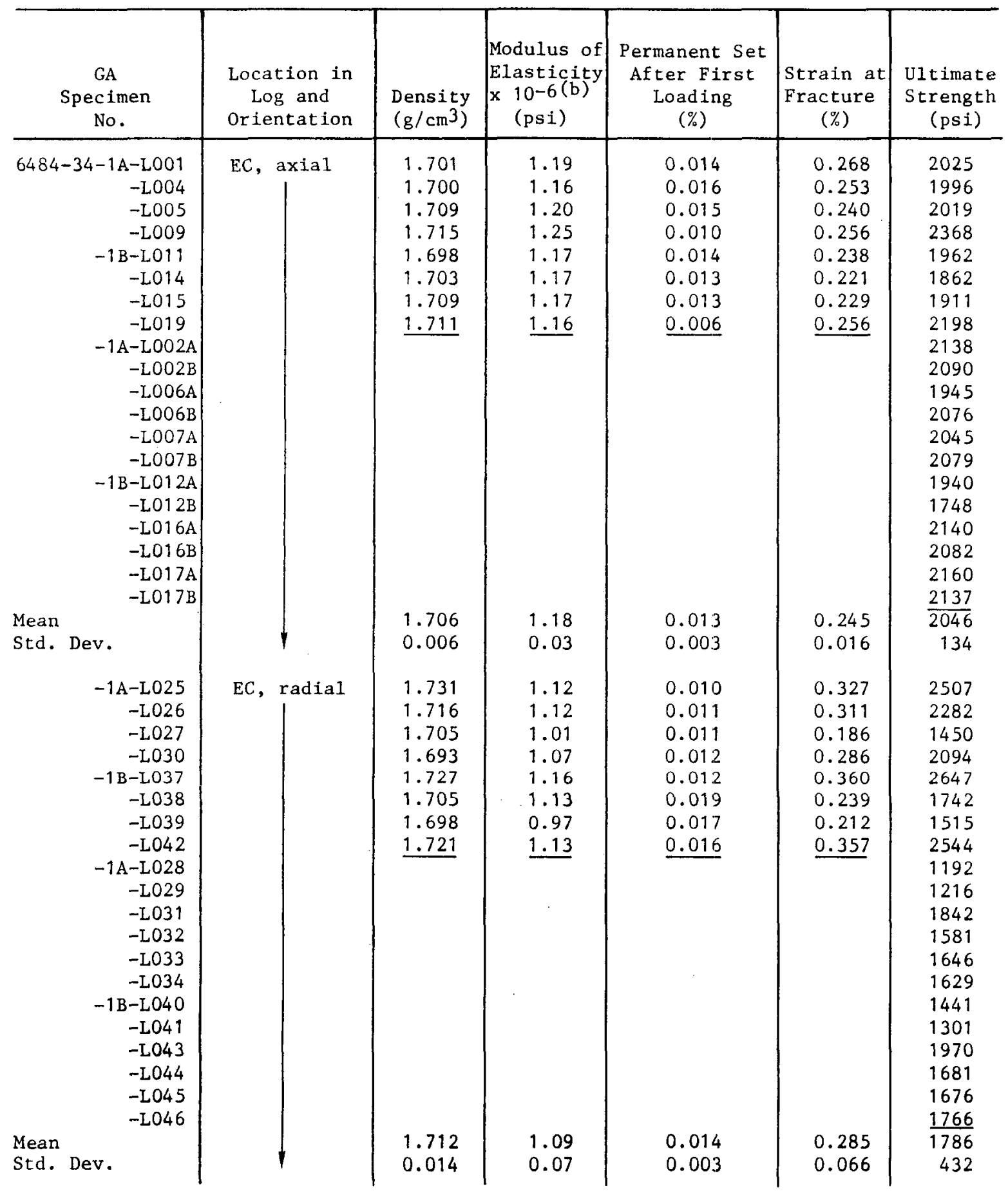


TABLE B-9 (Continued)

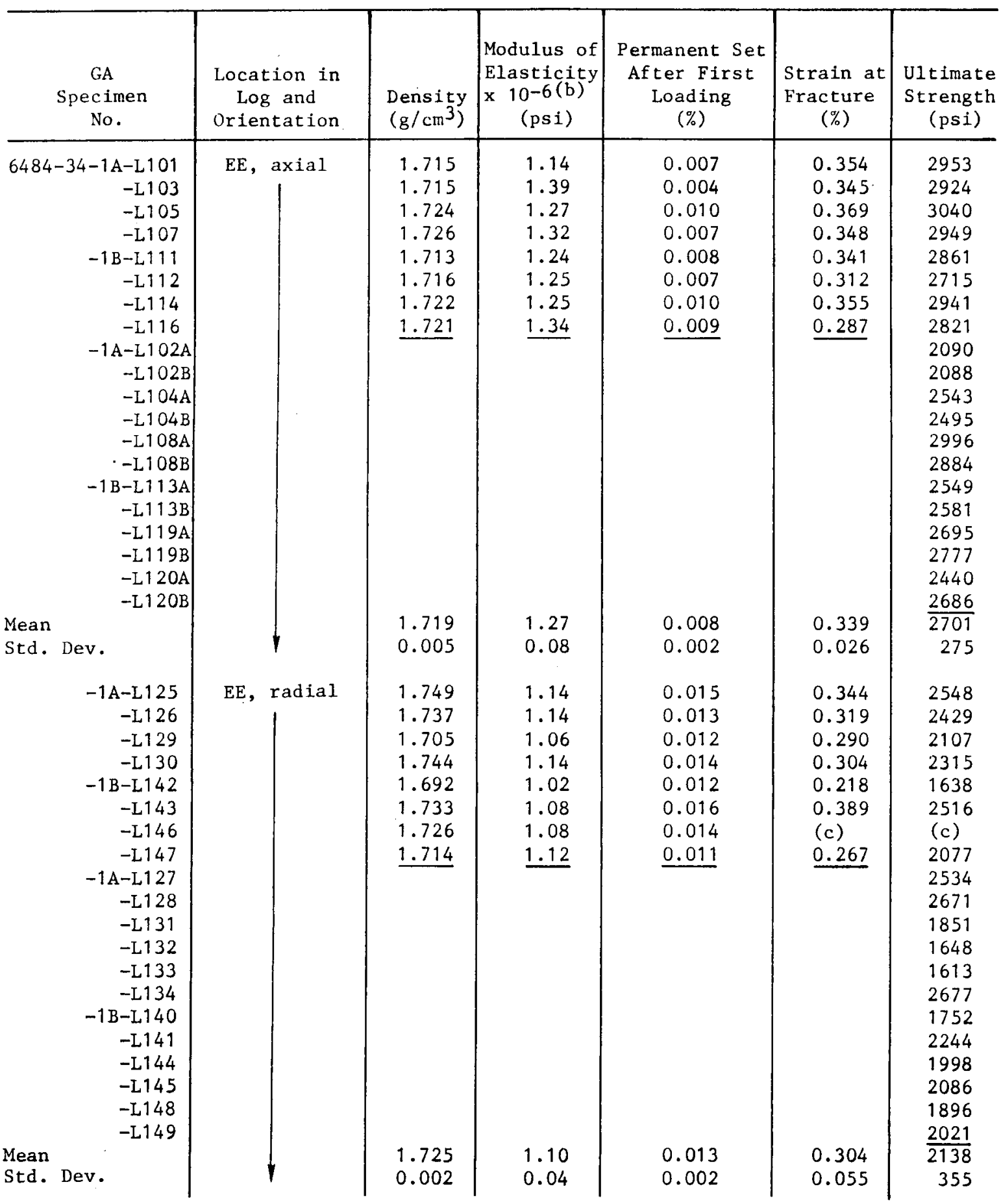


TABLE B-9 (Continued)

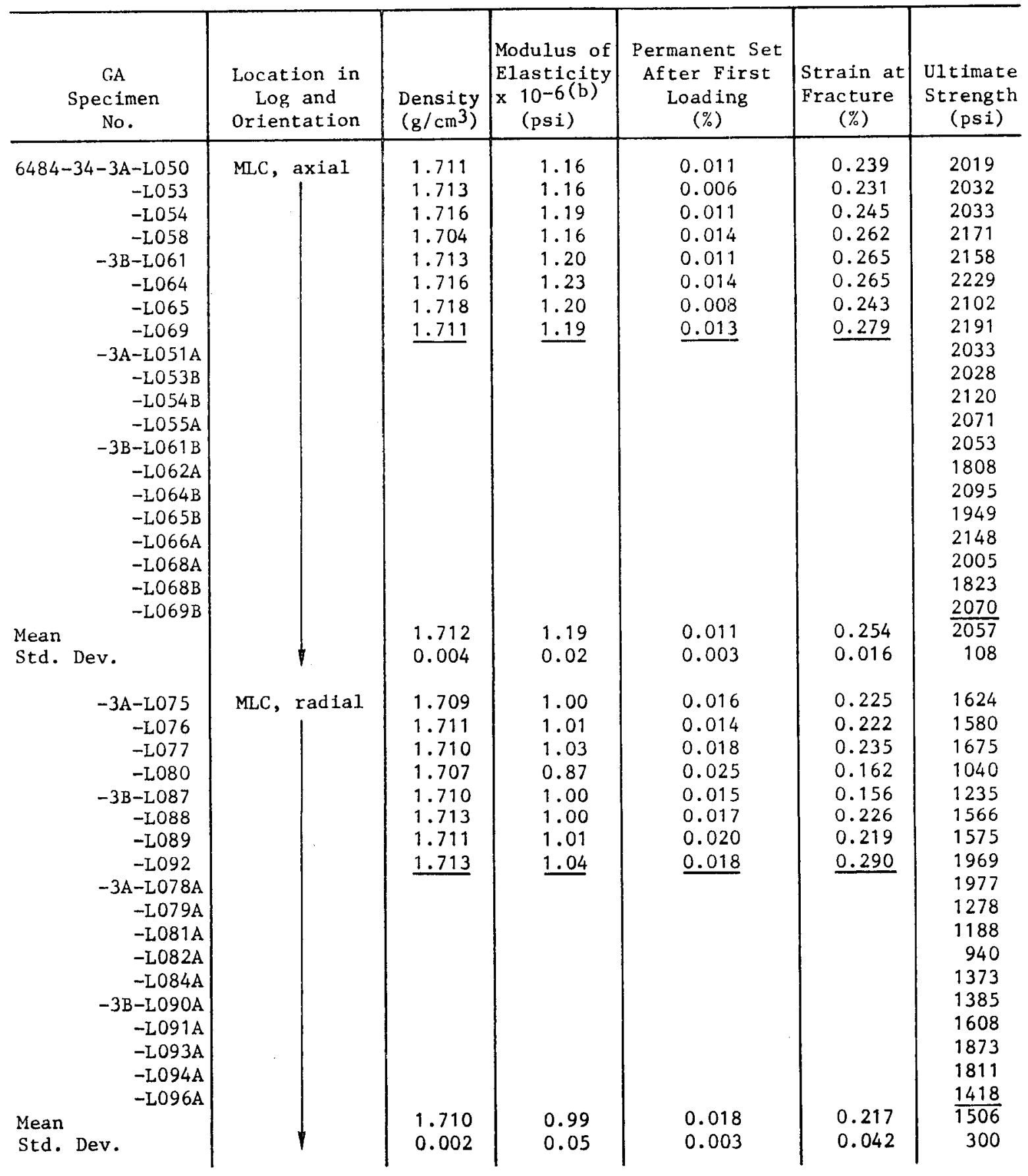


TABLE B-9 (Continued)

\begin{tabular}{|c|c|c|c|c|c|c|}
\hline $\begin{array}{c}\text { GA } \\
\text { Specimen } \\
\text { No. }\end{array}$ & $\begin{array}{l}\text { Location in } \\
\text { Log and } \\
\text { orientation }\end{array}$ & $\begin{array}{l}\text { Density. } \\
\left(\mathrm{g} / \mathrm{cm}^{3}\right)\end{array}$ & $\begin{array}{c}\text { Modulus of } \\
\text { Elasticity } \\
\times 10^{-6}(\mathrm{~b}) \\
(\mathrm{psi})\end{array}$ & $\begin{array}{c}\text { Permanent Set } \\
\text { After First } \\
\text { Loading } \\
(\%)\end{array}$ & $\begin{array}{c}\text { Strain at } \\
\text { Fracture } \\
(\%)\end{array}$ & $\begin{array}{l}\text { Ultimate } \\
\text { Strength } \\
\text { (psi) }\end{array}$ \\
\hline 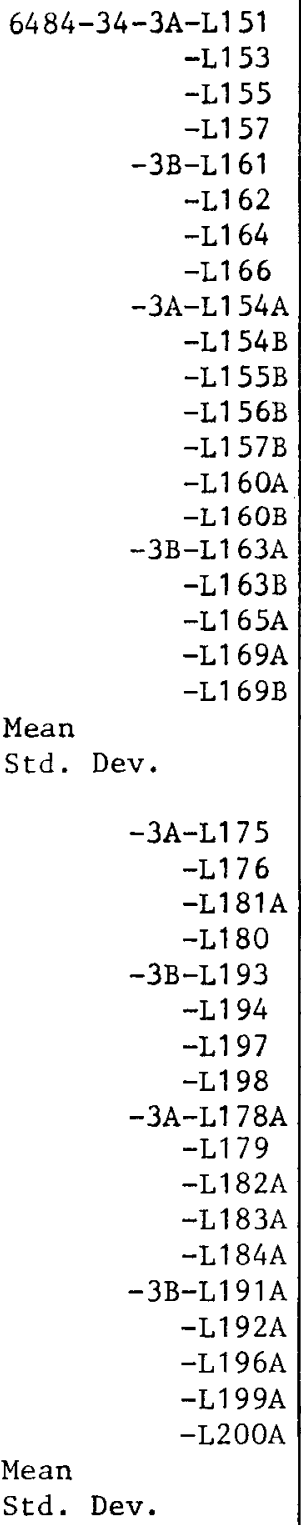 & MLE, radial & $\begin{array}{l}1.733 \\
0.007 \\
1.712 \\
1.723 \\
1.702 \\
1.710 \\
1.718 \\
1.708 \\
1.707 \\
1.709 \\
\end{array}$ & $\begin{array}{l}1.31 \\
0.03 \\
1.36 \\
1.10 \\
0.99 \\
1.06 \\
1.10 \\
1.19 \\
1.08 \\
1.09 \\
\end{array}$ & $\begin{array}{l}0.010 \\
0.001 \\
0.013 \\
0.013 \\
0.014 \\
0.013 \\
0.017 \\
0.014 \\
0.011 \\
0.012 \\
\end{array}$ & $\begin{array}{l}0.351 \\
0.023 \\
0.335 \\
0.345 \\
0.261 \\
0.237 \\
0.283 \\
0.199 \\
0.294 \\
0.244 \\
\end{array}$ & $\begin{array}{r}3120 \\
2854 \\
2994 \\
3282 \\
2812 \\
2881 \\
3000 \\
3094 \\
2446 \\
2243 \\
3039 \\
2841 \\
3077 \\
2926 \\
2696 \\
2580 \\
2597 \\
2715 \\
2680 \\
2520 \\
2817 \\
260 \\
\\
2430 \\
2494 \\
1870 \\
1823 \\
2154 \\
1570 \\
2190 \\
1903 \\
2499 \\
1332 \\
1287 \\
1436 \\
2145 \\
2617 \\
2262 \\
1599 \\
2292 \\
1845 \\
1986 \\
419\end{array}$ \\
\hline
\end{tabular}

(a) Measured at GLCC.

(b) Specimens were loaded to $1000 \mathrm{psi}$, unloaded to zero stress, and reloaded to failure while recording the stress-strain curve. Modulus of elasticity = chord modulus between 100 and 1000 psi on second loading.

(c) Bond failure. 
TABLE B-10

DENSITY AND TENSILE PROPERTIES OF H-451, LOT 426

[ $\log 6484-40$ (GLCC 155), whole $\log$ density $=1.72 \mathrm{~g} / \mathrm{cm}^{3}{ }^{(\mathrm{a})}$,

0.505-in.-diameter by 3.00-in.-long specimens]

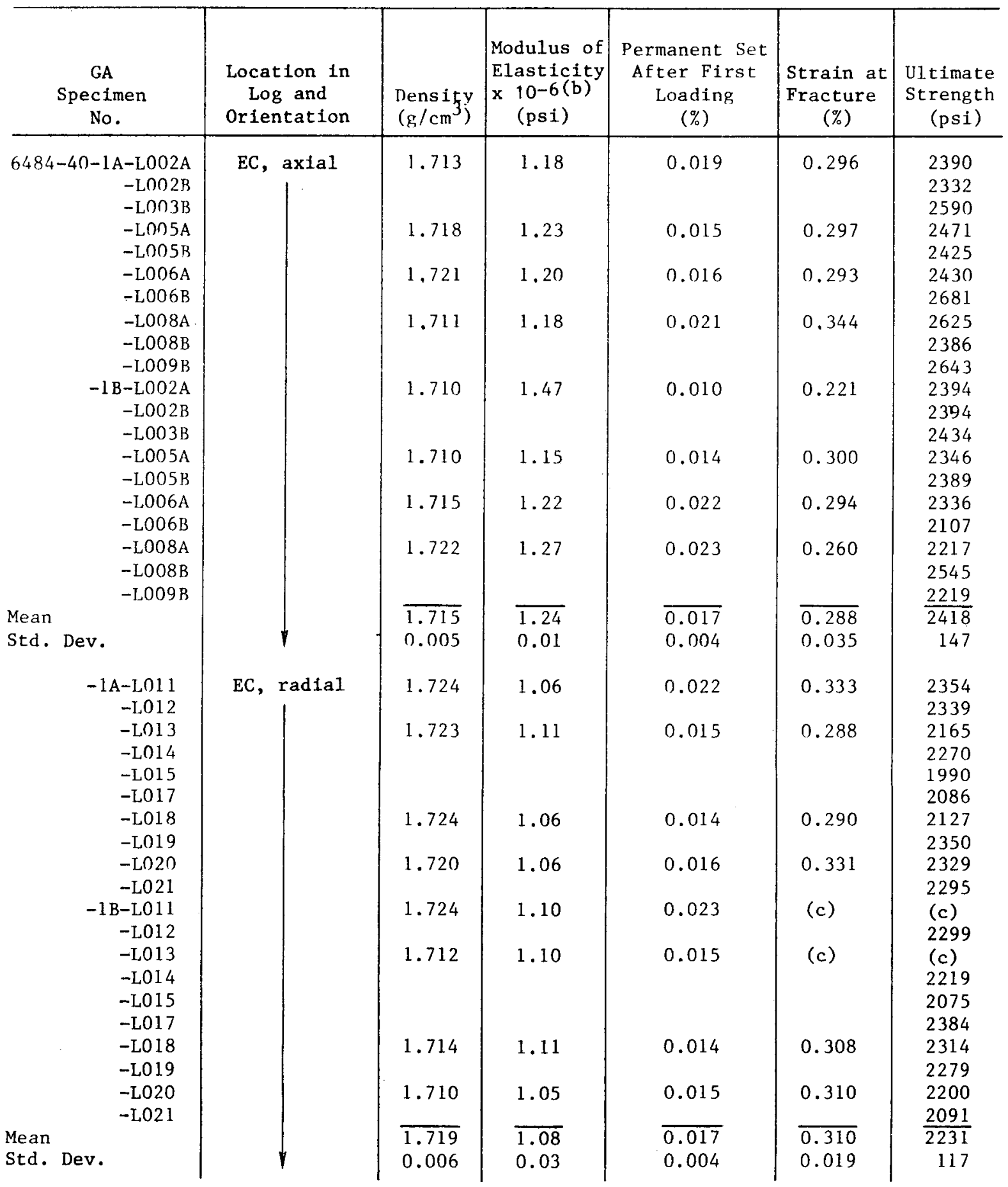


TABLE B-10 (Continued)

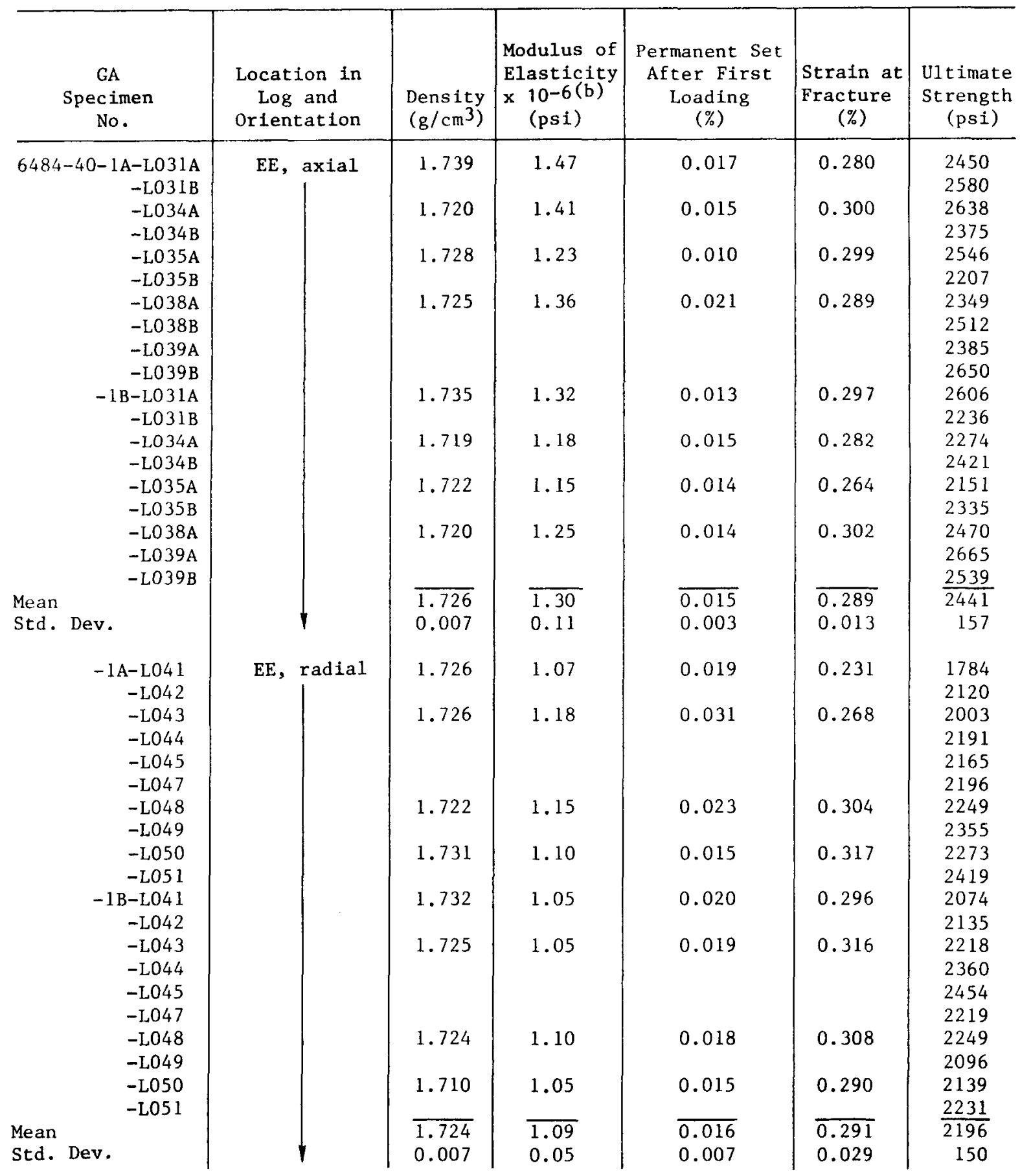


TABLE B-10 (Continued)

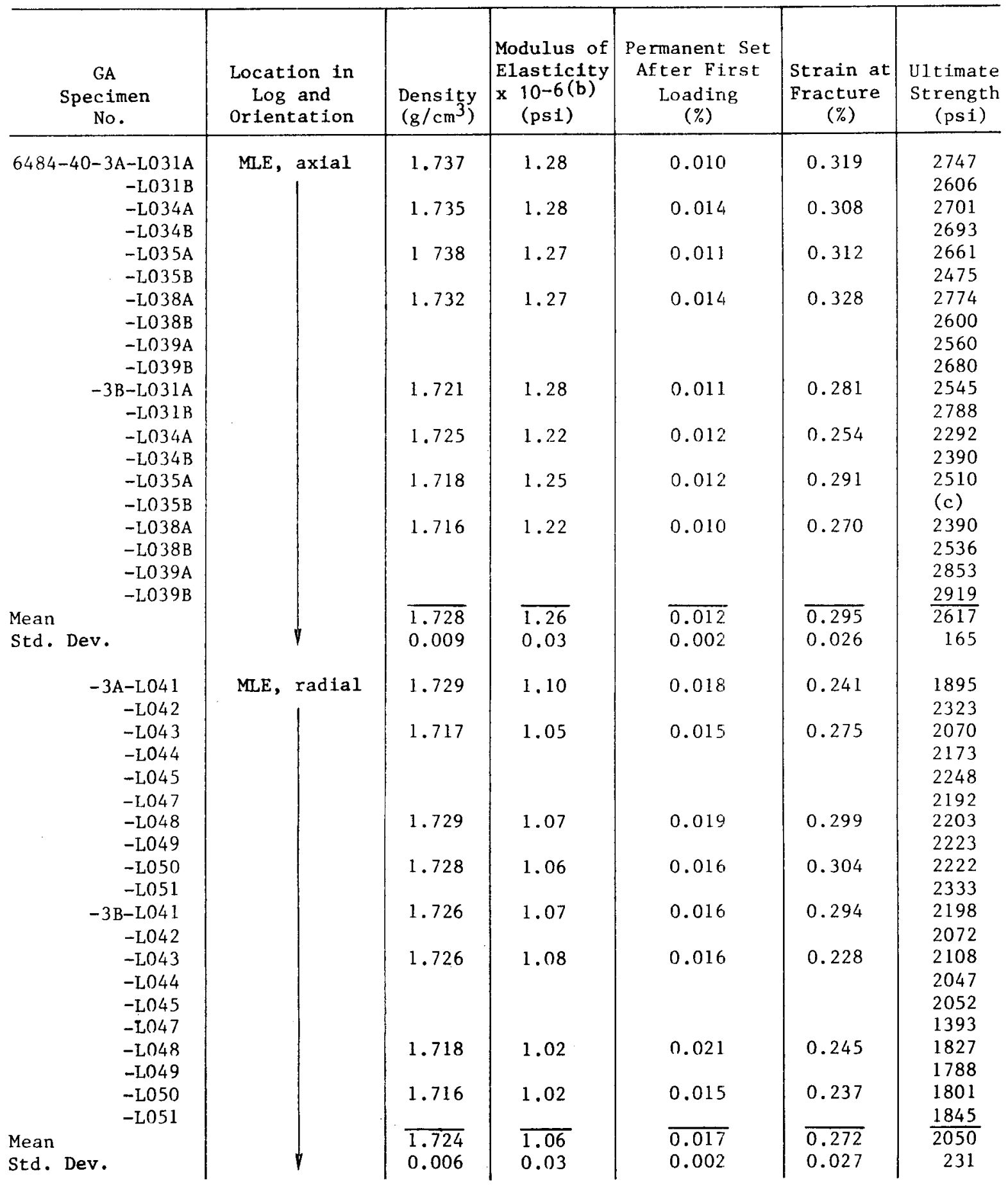


TABLE B-10 (Continued)

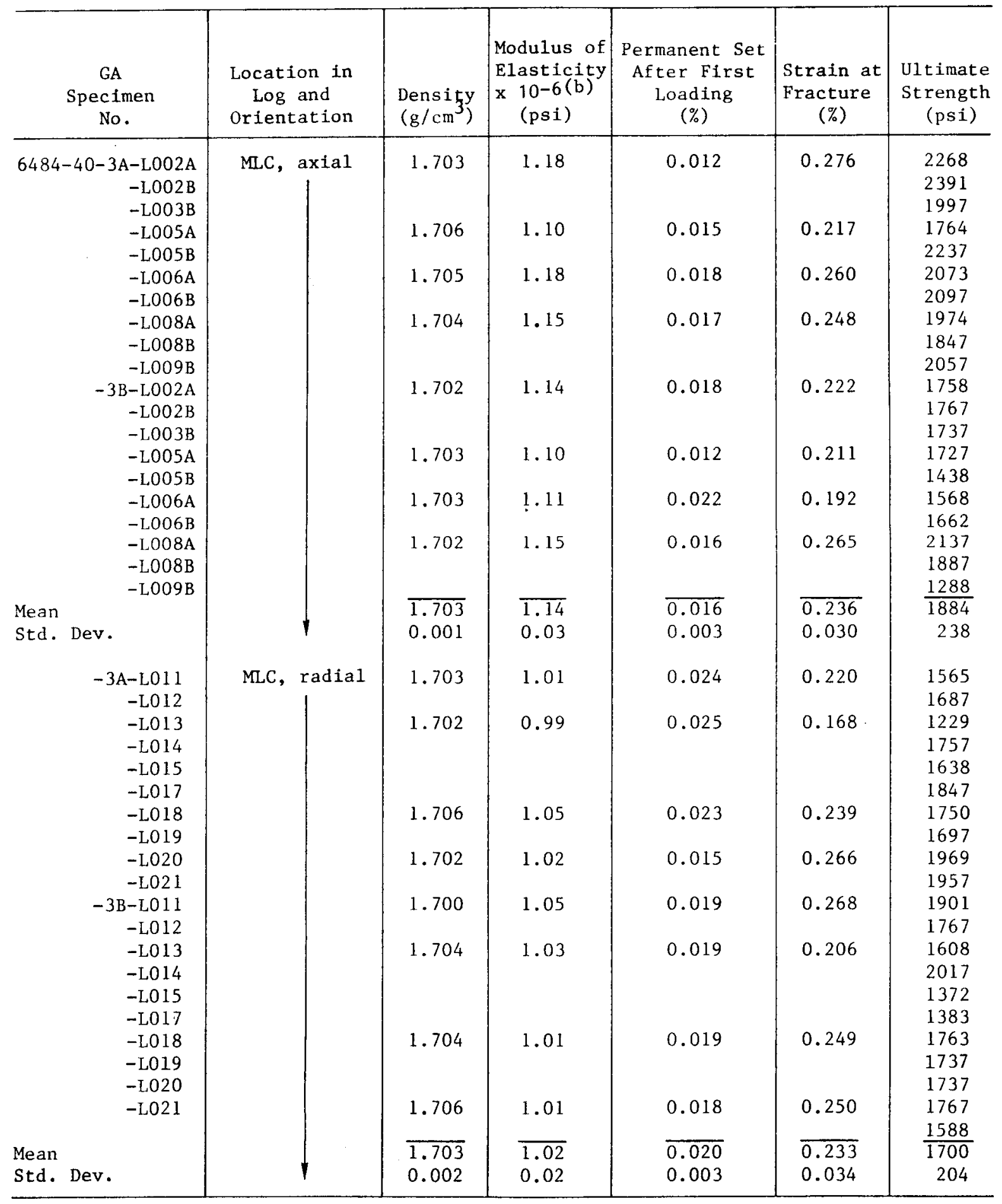

(a) Measured at GLCC.

(b) Specimens were loaded to $1000 \mathrm{psi}$, unloaded to zero stress, and reloaded to failure while recording the stress-strain curve. Modulus of elasticity $=$ chord modulus between 100 and 1000 psi on second loading.

(c) Bond failure. 
TABLE B-11

DENSITY AND TENSILE PROPERTIES OF H-451, LOT 426 [ $\log 6484-41$ (GLCC. 184), whole $\log$ density $=1.72 \mathrm{~g} / \mathrm{cm}^{3}{ }^{(a)}$, 0.505-in.-diameter by 3.00-in.-1ong specimens]

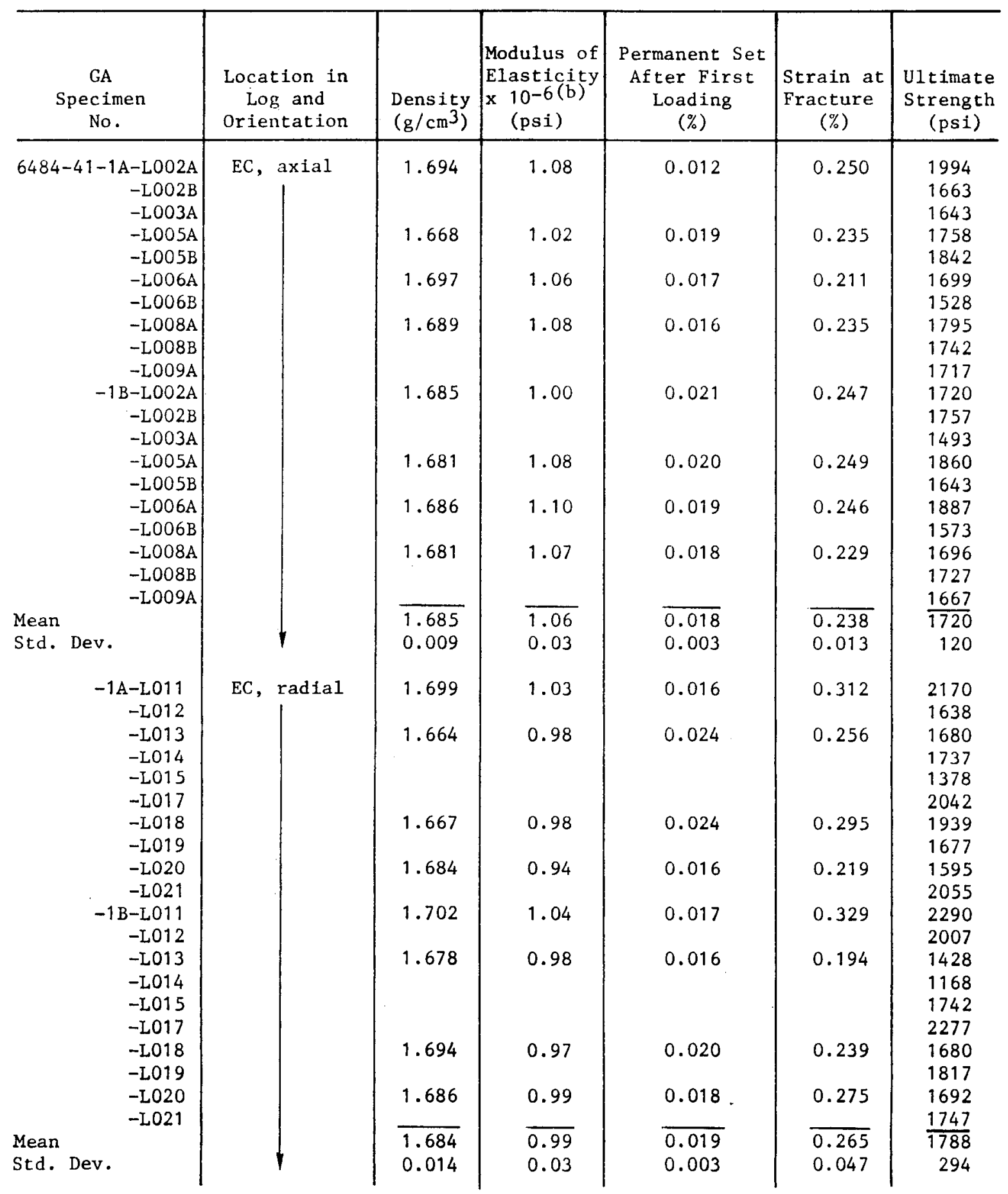


TABLE B-11 (Continued)

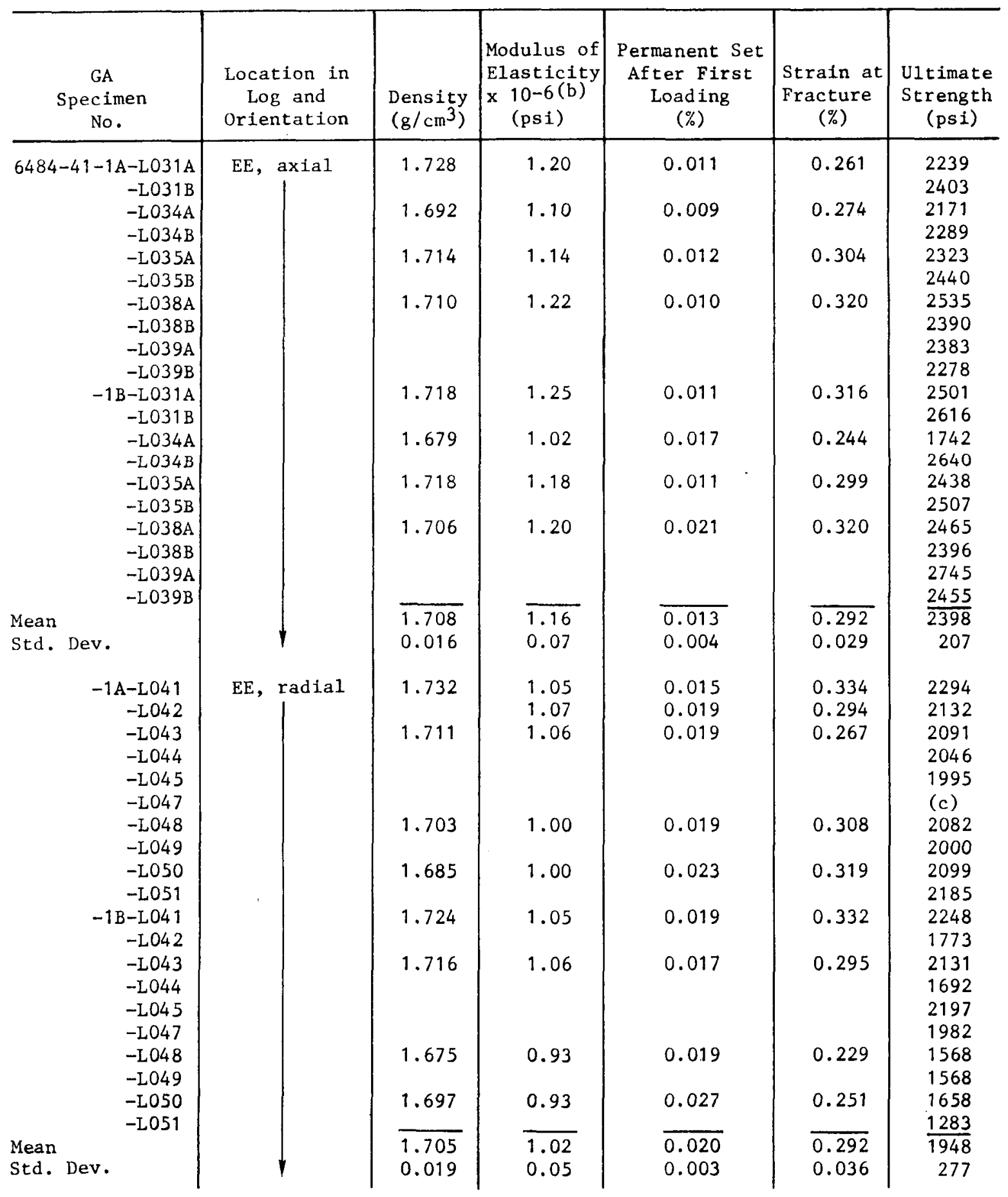


TABLE B-11 (Continued)

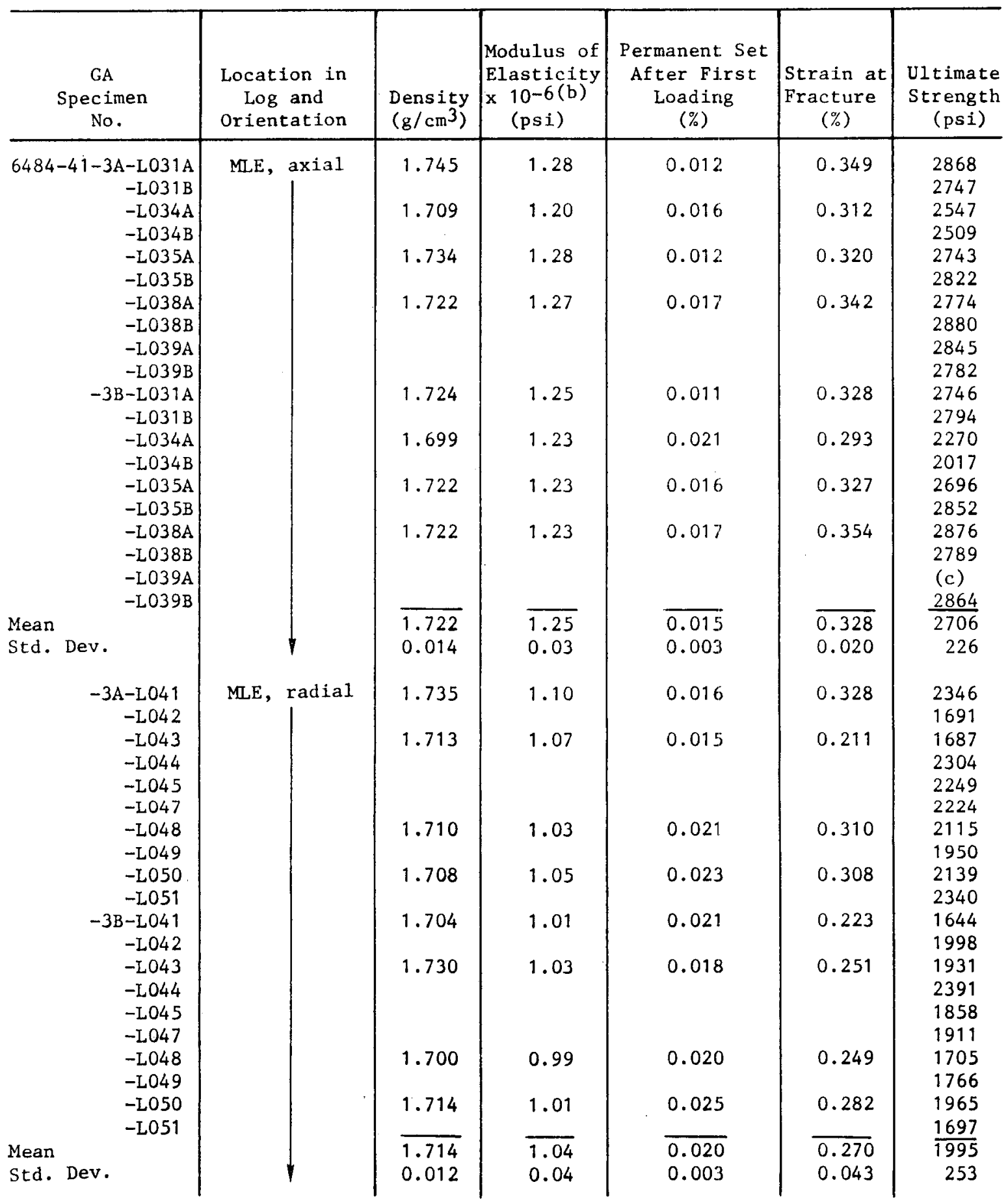


TABLE B-11 (Continued)

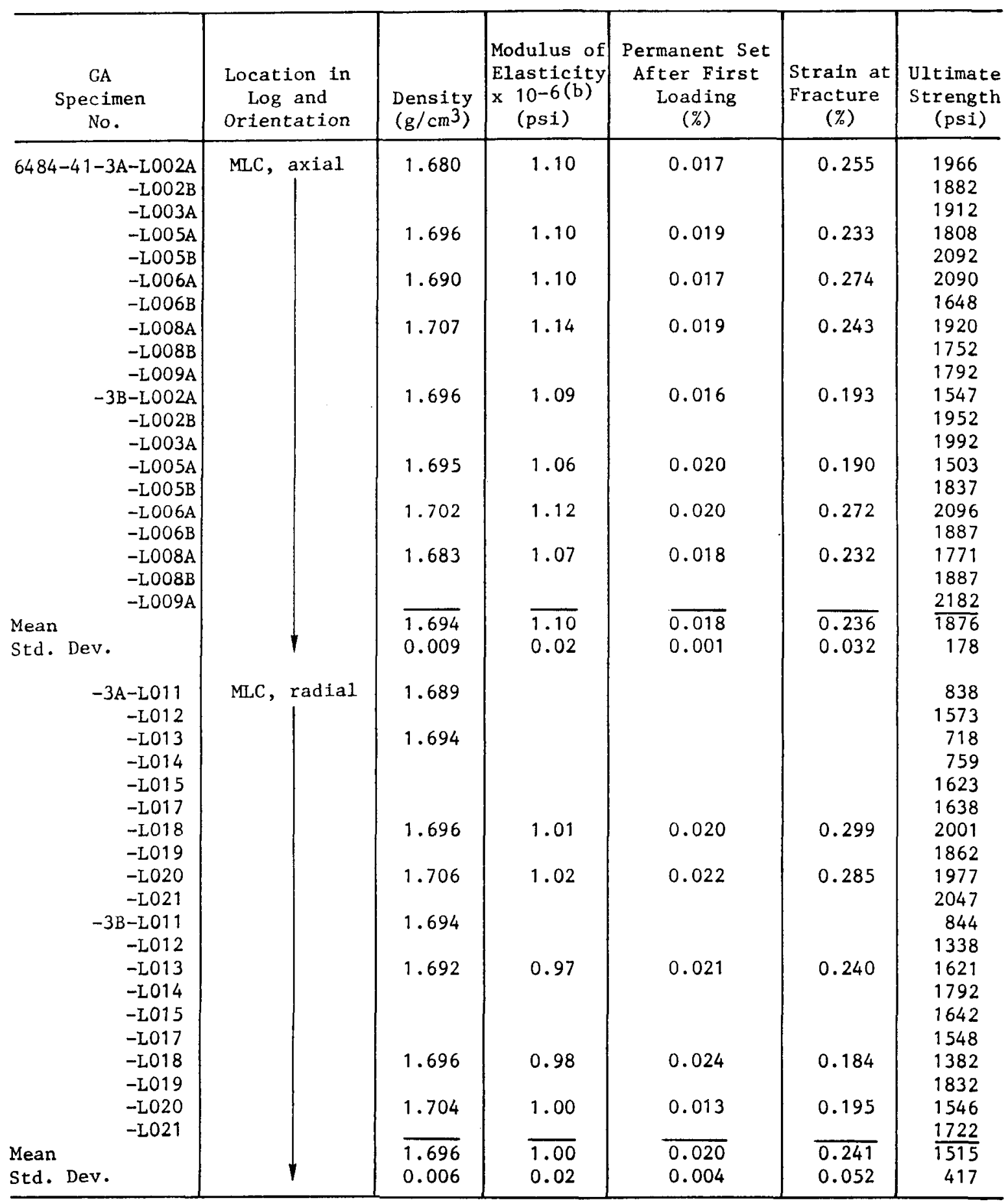

(a) Measured at GLCC.

(b) Specimens were loaded to 1000 psi, unloaded to zero stress, and reloaded to fallure while recording the stress-strain curve. Modulus of elasticity = chord modulus between 100 and $1000 \mathrm{psi}$ on second loading.

(c) Bond failure. 
TABLE B-12

DENSITY AND TENSILE PROPERTIES OF TS-1240, LOT 1

$\left[\log 5651-72\right.$ (UCC 32), whole $\log$ density $=1.77 \mathrm{~g} / \mathrm{cm}^{3}$ (a), 0.505-in.-diameter by 3.0-in.-long specimens]

\begin{tabular}{|c|c|c|c|c|c|c|}
\hline $\begin{array}{l}\text { GA } \\
\text { Specimen } \\
\text { No. }\end{array}$ & $\begin{array}{l}\text { Location in } \\
\text { Log and } \\
\text { Orientation }\end{array}$ & $\begin{array}{l}\text { Density } \\
\left(\mathrm{g} / \mathrm{cm}^{3}\right)\end{array}$ & $\begin{array}{l}\text { Modulus of } \\
\text { Elasticity } \\
x \quad 10-6(\mathrm{~b}) \\
\quad(\mathrm{psi})\end{array}$ & $\begin{array}{c}\text { Permanent Set } \\
\text { After First } \\
\text { Loading } \\
(\%) \\
\end{array}$ & $\begin{array}{c}\text { Strain at } \\
\text { Fracture } \\
(\%)\end{array}$ & $\begin{array}{l}\text { Ultimate } \\
\text { Strength } \\
\text { (psi) }\end{array}$ \\
\hline 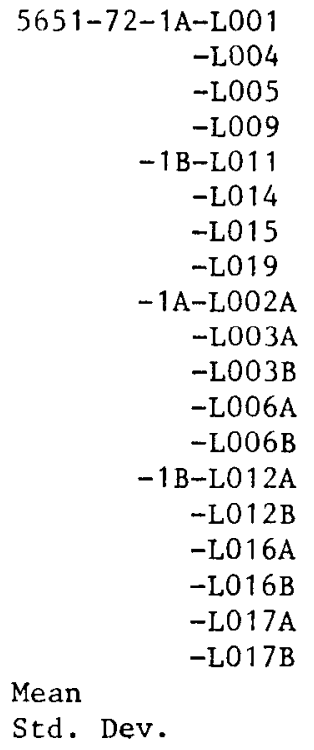 & EC, axial & $\begin{array}{l}1.736 \\
1.721 \\
1.700 \\
1.732 \\
1.727 \\
1.719 \\
1.711 \\
1.734 \\
\end{array}$ & $\begin{array}{l}1.07 \\
0.94 \\
0.82 \\
0.92 \\
0.99 \\
1.00 \\
0.80 \\
0.93 \\
\end{array}$ & $\begin{array}{l}-- \\
-- \\
-- \\
-- \\
-- \\
-- \\
--\end{array}$ & $\begin{array}{l}0.260 \\
0.170 \\
0.156 \\
0.304 \\
0.234 \\
0.176 \\
0.266 \\
0.227 \\
\end{array}$ & $\begin{array}{r}1980 \\
1443 \\
1210 \\
1941 \\
1742 \\
1454 \\
1618 \\
1661 \\
1347 \\
1302 \\
1429 \\
1203 \\
1152 \\
1538 \\
1756 \\
1401 \\
1554 \\
1350 \\
1293 \\
1493 \\
240\end{array}$ \\
\hline 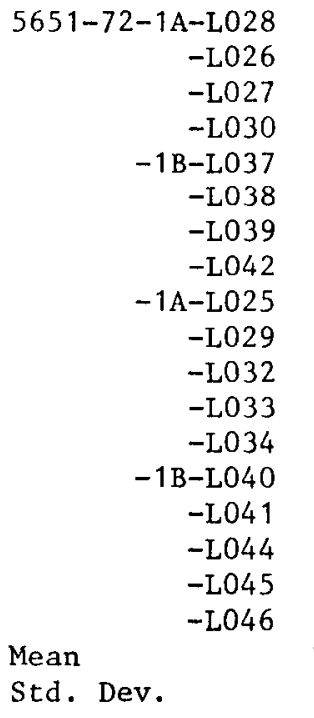 & EC, radial & $\begin{array}{l}1.706 \\
1.744 \\
1.722 \\
1.724 \\
1.731 \\
1.723 \\
1.726 \\
1.722 \\
1.753 \\
\end{array}$ & $\begin{array}{l}0.84 \\
1.02 \\
0.95 \\
1.05 \\
0.96 \\
0.95 \\
1.02 \\
0.98 \\
1.01 \\
\end{array}$ & $\begin{array}{l}0.030 \\
-- \\
-- \\
-- \\
-- \\
-- \\
-- \\
-- \\
-- \\
\end{array}$ & $\begin{array}{l}0.363 \\
0.308 \\
0.326 \\
0.200 \\
0.312 \\
0.295 \\
0.288 \\
0.310 \\
\text { (c) } \\
\end{array}$ & $\begin{array}{l}1909 \\
2179 \\
2079 \\
1776 \\
1979 \\
1964 \\
2127 \\
2071 \\
(c) \\
1920 \\
1892 \\
1581 \\
1609 \\
2077 \\
249 \\
1936 \\
728 \\
2104 \\
1775 \\
519\end{array}$ \\
\hline
\end{tabular}


TABLE B-12 (Continued)

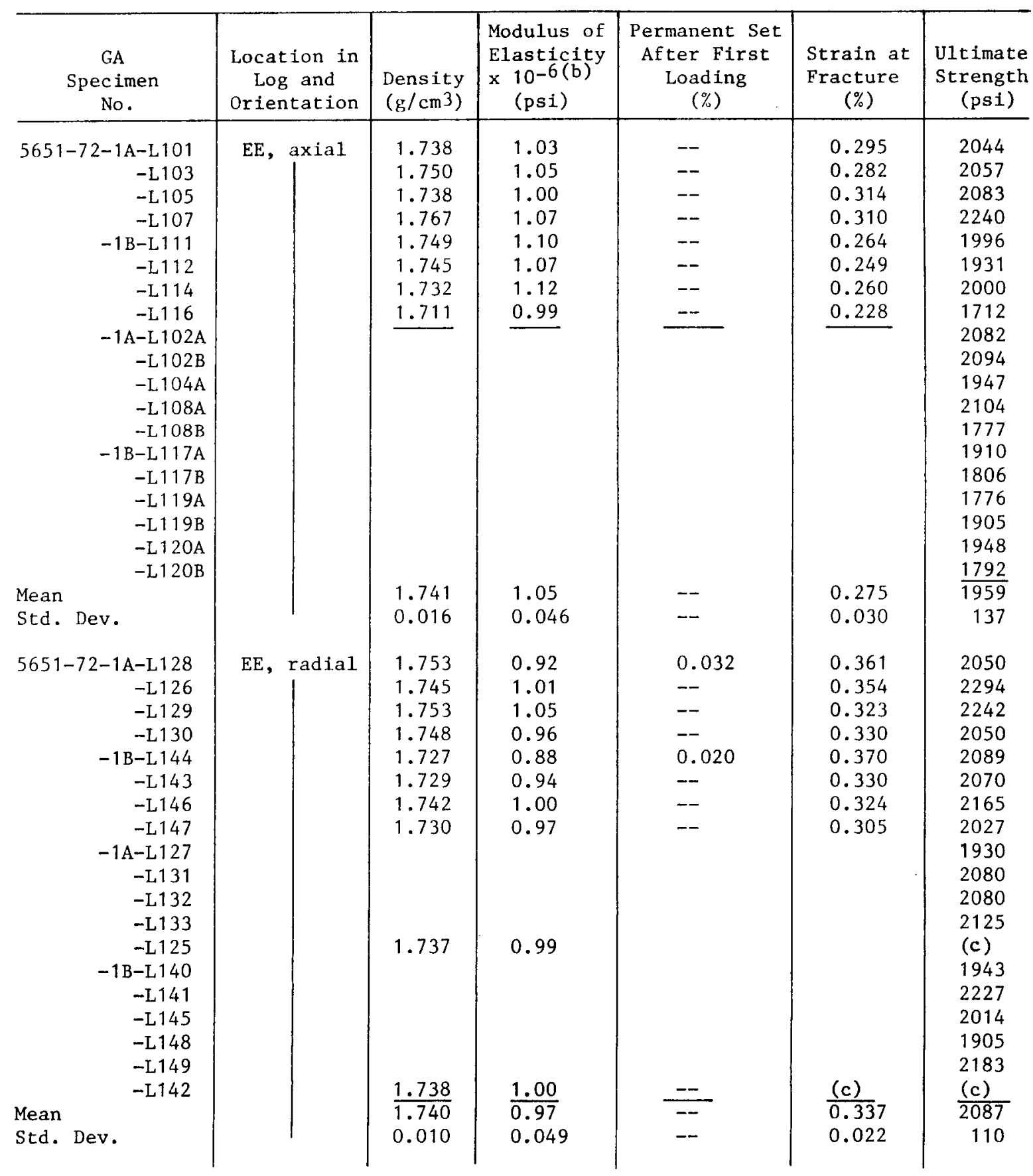


TABLE B-12 (Continued)

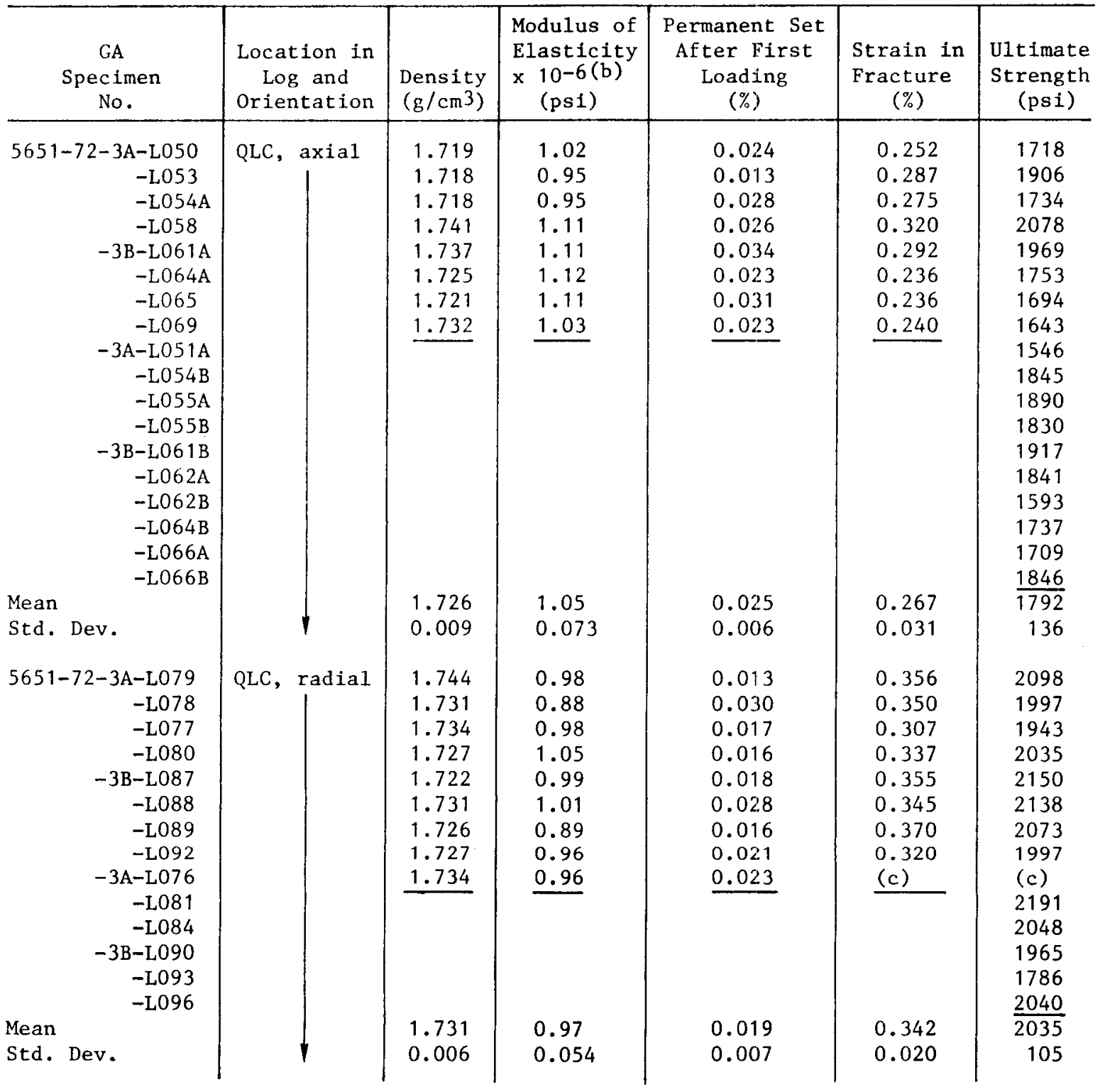


TABLE B-12 (Continued)

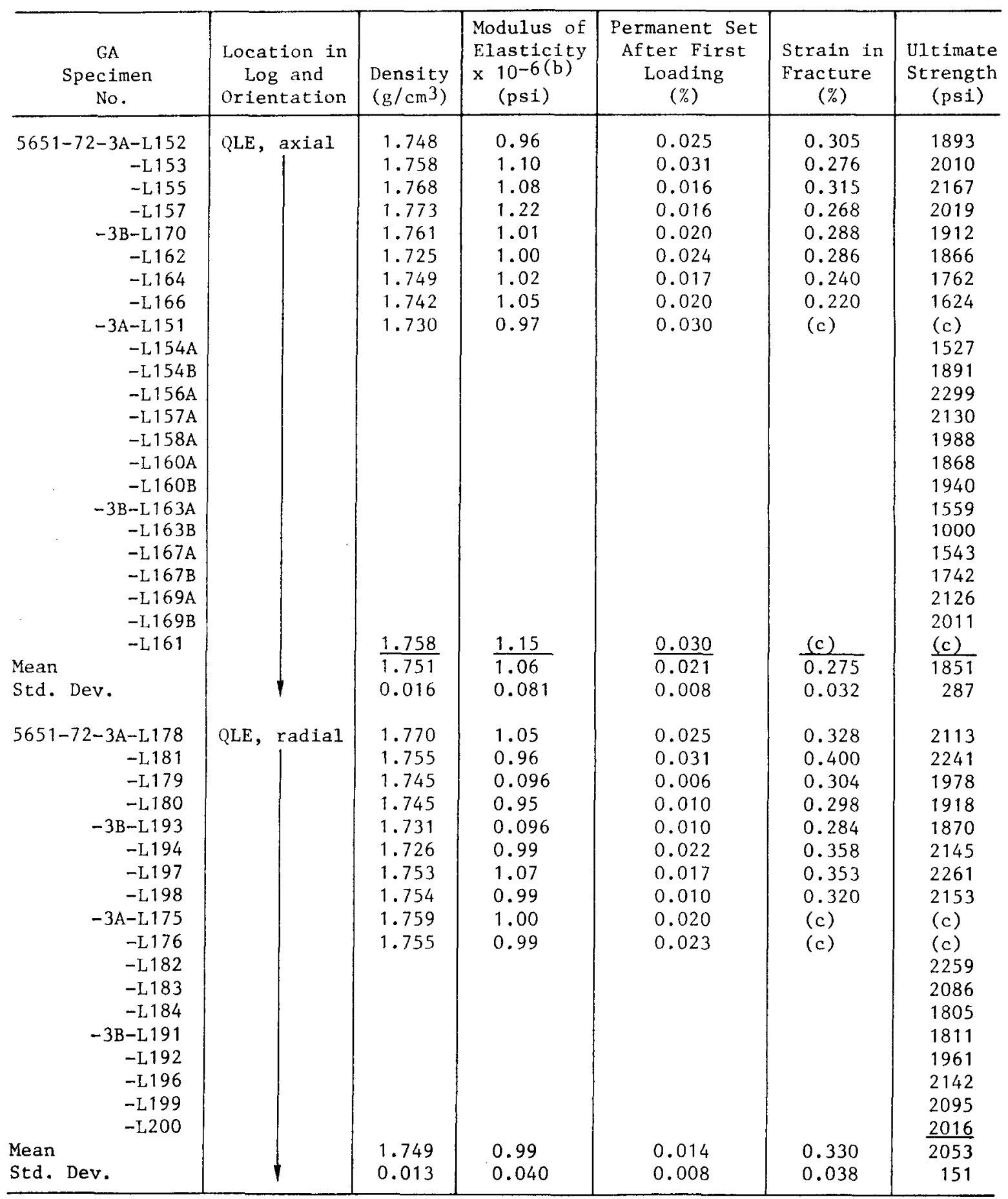

(a) Measured at UCC.

(b) Specimens were loaded to $1000 \mathrm{psi}$, unloaded to zero stress, and reloaded to failure while recordlng the stress-strain curve. Modulus of elasticity $=$ chord modulus between 100 and 1000 psi on second loading.

(c) Bond failure. 
TABLE B-13

DENSITY AND TENSILE PROPERTIES OF TS-1240, LOT 1

$\left[\log 5651-73\right.$ (UCC 40), whole $\log$ density $=1.79 \mathrm{~g} / \mathrm{cm}^{3}$ (a), 0.505-in.-diameter by 3.0-in.-long specimens]

\begin{tabular}{|c|c|c|c|c|c|c|}
\hline $\begin{array}{c}\text { GA } \\
\text { Specimen } \\
\text { No. }\end{array}$ & $\begin{array}{l}\text { Location in } \\
\text { Log and } \\
\text { orientation }\end{array}$ & $\begin{array}{r}\text { Density } \\
\left(\mathrm{g} / \mathrm{cm}^{3}\right)\end{array}$ & $\begin{array}{l}\text { Modulus of } \\
\text { E1asticity } \\
\times 10-6(b) \\
\quad \text { (psi) }\end{array}$ & $\begin{array}{c}\text { Permanent Set } \\
\text { After First } \\
\text { Loading } \\
(\%)\end{array}$ & $\begin{array}{c}\text { Strain at } \\
\text { Fracture } \\
(\%)\end{array}$ & $\begin{array}{l}\text { U1timate } \\
\text { Strength } \\
\quad \text { (psi) }\end{array}$ \\
\hline $5651-73-3 A-L 002 A$ & MLC, axial & 1.783 & 0.97 & 0.009 & 0.242 & 1837 \\
\hline$-\mathrm{L} 002 \mathrm{~B}$ & 1 & 1.782 & 1.00 & 0.008 & 0.255 & 1873 \\
\hline$-\mathrm{L} 003 \mathrm{~A}$ & & 1.777 & 1.00 & 0.007 & 0.230 & 1658 \\
\hline$-\mathrm{L} 003 \mathrm{~B}$ & & 1.779 & 1.02 & 0.010 & 0.230 & 1724 \\
\hline$-3 B-L 002 A$ & & 1.784 & 1.03 & 0.010 & 0.231 & 1714 \\
\hline$-L 002 B$ & & 1.791 & 1.10 & 0.012 & 0.258 & 1969 \\
\hline Mean & & 1.783 & 1.02 & $\overline{0.009}$ & $\overline{0.241}$ & $\overline{1796}$ \\
\hline Std. Dev. & 1 & 0.005 & 0.044 & 0.002 & 0.013 & 117 \\
\hline $5651-73-3 A-L 005$ & MLC, radial & 1.788 & 1.00 & 0.000 & 0.185 & 1452 \\
\hline$-\mathrm{L} 006$ & & 1.789 & 0.97 & 0.005 & 0.227 & 1665 \\
\hline$-\mathrm{L} 007$ & & 1.783 & (c) & (c) & (c) & 1316 \\
\hline-2008 & & 1.779 & 1.00 & 0.017 & 0.158 & 1233 \\
\hline$-3 B-L 005$ & & 1.786 & 1.22 & 0.020 & 0.208 & 1560 \\
\hline$-\mathrm{L} 007$ & & 1.789 & 1.02 & 0.017 & 0.192 & 1388 \\
\hline Mean & & $\overline{1.786}$ & 1.04 & $\overline{0.012}$ & $\overline{0.194}$ & $\overline{1436}$ \\
\hline Std. Dev. & $\gamma$ & 0.004 & 0.101 & 0.009 & 0.026 & 159 \\
\hline $5651-73-3 A-L 009 A$ & MLE, axial & 1.814 & 1.18 & 0.018 & 0.342 & 2583 \\
\hline- L011A & & 1.800 & 1.12 & 0.030 & 0.317 & 2287 \\
\hline$-\mathrm{L} 010 \mathrm{~A}$ & & 1.814 & 1.15 & 0.015 & 0.365 & 2684 \\
\hline$-\mathrm{L} 009 \mathrm{~B}$ & & 1.795 & (c) & (c) & $(c, d)$ & (d) \\
\hline$-\mathrm{L} 010 \mathrm{~B}$ & & 1.815 & (c) & (c) & $(c, d)$ & (d) \\
\hline$-3 B-L 011 A$ & & 1.808 & 1.10 & 0.031 & (d) & (d) \\
\hline$-\mathrm{L} 011 \mathrm{~B}$ & & 1.810 & 1.34 & 0.030 & (d) & (d) \\
\hline$-L 009 A$ & & 1.806 & 1.17 & 0.016 & 0.255 & 2204 \\
\hline$-\mathrm{L} 009 \mathrm{~B}$ & & 1.800 & 1.15 & 0.014 & 0.345 & 2498 \\
\hline Mean & & $\overline{1.807}$ & $\overline{1.17}$ & $\overline{0.022}$ & $\overline{0.327}$ & 2290 \\
\hline Std. Dev. & 1 & 0.007 & 0.079 & 0.008 & 0.049 & 357 \\
\hline $5651-73-3 A-L 013$ & MLE, radial & 1.808 & 0.99 & 0.015 & 0.292 & 2004 \\
\hline$-\mathrm{LO} 14$ & & 1.801 & 1.01 & 0.019 & 0.257 & 1730 \\
\hline$-\mathrm{L} 015$ & & 1.804 & 1.02 & 0.016 & 0.255 & 1755 \\
\hline$-3 B-L 013$ & & 1.799 & 1.03 & 0.019 & 0.285 & 1939 \\
\hline$-\mathrm{L} 014$ & & 1.804 & 1.07 & 0.012 & 0.248 & 1790 \\
\hline$-\mathrm{L} 015$ & & 1.804 & 1.18 & 0.016 & 0.260 & 1805 \\
\hline Mean & & 1.803 & 1.05 & 0.016 & 0.266 & 1837 \\
\hline Std. Dev. & $\gamma$ & 0.003 & 0.069 & 0.003 & 0.018 & 109 \\
\hline
\end{tabular}

(a) Measured at UCC.

(b) Specimens were loaded to $1000 \mathrm{psi}$, unloaded to zero stress and reloaded to failure while recording the stress-strain curve. Modulus of elasticity = chord modulus between 100 and 1000 psi on second loading.

(c) Specimen tested without extensometer and not load cycled.

(d) Bond failure. 
TABLE $B-14$

DENSITY AND TENSILE PROPERTIES OF TS -1240 , LOT 1

$\left[\log 5651-73\right.$ (UCC 40), whole $\log$ density $=1.79 \mathrm{~g} / \mathrm{cm}^{3}{ }^{(a)}$, 0.25-in.-diameter by 0.9-in.-long specimens]

\begin{tabular}{|c|c|c|c|c|c|c|}
\hline $\begin{array}{c}\text { GA } \\
\text { Specimen } \\
\text { No. }\end{array}$ & $\begin{array}{l}\text { Location in } \\
\text { Log and } \\
\text { Orientation }\end{array}$ & $\begin{array}{l}\text { Density } \\
\left(\mathrm{g} / \mathrm{cm}^{3}\right)\end{array}$ & $\begin{array}{l}\text { Modulus of } \\
\text { Elasticity } \\
\times 10-6(b) \\
\quad(p s i)\end{array}$ & $\begin{array}{c}\text { Permanent Set } \\
\text { After First } \\
\text { Loading } \\
(\%)\end{array}$ & $\begin{array}{c}\text { Strain at } \\
\text { Fracture } \\
(\%)\end{array}$ & $\begin{array}{l}\text { Ultimate } \\
\text { Strength } \\
\quad \text { (psi) }\end{array}$ \\
\hline $\begin{array}{r}5651-73-3 \mathrm{~A}-106 \mathrm{~B} \\
-107 \mathrm{~B} \\
-112 \mathrm{~B} \\
-113 \mathrm{~B} \\
-118 \mathrm{~B} \\
-119 \mathrm{~B} \\
-124 \mathrm{~B} \\
-125 \mathrm{~B} \\
-127 \mathrm{~B} \\
-128 \mathrm{~B} \\
-3 \mathrm{~B}-106 \mathrm{~B} \\
-107 \mathrm{~B} \\
-112 \mathrm{~B} \\
-113 \mathrm{~B} \\
-118 \mathrm{~B} \\
-119 \mathrm{~B} \\
-124 \mathrm{~B} \\
-125 \mathrm{~B} \\
-127 \mathrm{~B} \\
-128 \mathrm{~B}\end{array}$ & MLE, axial & $\begin{array}{l}1.812 \\
1.811 \\
1.813 \\
1.796 \\
1.813 \\
1.798 \\
1.803 \\
1.801 \\
1.798 \\
1.801 \\
1.803 \\
1.801 \\
1.817 \\
1.809 \\
1.805 \\
1.800 \\
1.817 \\
1.820 \\
1.811 \\
1.820 \\
\end{array}$ & $\begin{array}{l}1.06 \\
1.02 \\
1.06 \\
1.03 \\
1.06 \\
0.98 \\
0.97 \\
0.99 \\
0.98 \\
1.01 \\
0.95 \\
1.00 \\
1.12 \\
1.20 \\
1.20 \\
1.06 \\
1.06 \\
1.06 \\
(\mathrm{c}) \\
1.29 \\
\end{array}$ & $\begin{array}{l}0.020 \\
0.006 \\
0.017 \\
0.007 \\
0.022 \\
0.032 \\
0.020 \\
0.010 \\
0.025 \\
0.030 \\
0.070 \\
0.035 \\
0.035 \\
0.015 \\
0.020 \\
0.030 \\
0.040 \\
0.030 \\
(\mathrm{c}) \\
0.010 \\
\end{array}$ & $\begin{array}{l}0.440 \\
0.365 \\
0.383 \\
0.318 \\
0.315 \\
0.375 \\
0.375 \\
0.355 \\
0.365 \\
0.356 \\
0.260 \\
0.290 \\
0.350 \\
0.390 \\
0.360 \\
0.410 \\
0.410 \\
0.380 \\
0.130 \\
0.370 \\
\end{array}$ & $\begin{array}{r}2789 \\
2569 \\
2400 \\
2262 \\
2120 \\
2267 \\
2244 \\
2347 \\
2183 \\
2042 \\
1540 \\
2082 \\
2526 \\
2648 \\
2588 \\
2648 \\
2699 \\
2608 \\
971 \\
2777 \\
\end{array}$ \\
\hline $\begin{array}{l}\text { Mean } \\
\text { Std. Dev. }\end{array}$ & 1 & $\begin{array}{l}1.807 \\
0.008\end{array}$ & $\begin{array}{l}1.06 \\
0.089\end{array}$ & $\begin{array}{l}0.025 \\
0.014\end{array}$ & $\begin{array}{l}0.350 \\
0.066\end{array}$ & $\begin{array}{r}2315 \\
440\end{array}$ \\
\hline $\begin{array}{r}5651-73-3 \mathrm{~A}-136 \mathrm{~B} \\
-137 \mathrm{~B} \\
-142 \mathrm{~B} \\
-143 \mathrm{~B} \\
-148 \mathrm{~B} \\
-149 \mathrm{~B} \\
-154 \mathrm{~B} \\
-155 \mathrm{~B} \\
-160 \mathrm{~A} \\
-160 \mathrm{~B} \\
-3 \mathrm{~B}-136 \mathrm{~B} \\
-137 \mathrm{~B} \\
-142 \mathrm{~B} \\
-143 \mathrm{~B} \\
-148 \mathrm{~B} \\
-149 \mathrm{~B} \\
-154 \mathrm{~B} \\
-155 \mathrm{~B} \\
-160 \mathrm{~A} \\
-160 \mathrm{~B}\end{array}$ & MLE, radial & $\begin{array}{l}1.794 \\
1.804 \\
1.812 \\
1.812 \\
1.820 \\
1.807 \\
1.808 \\
1.806 \\
1.799 \\
1.813 \\
1.814 \\
1.815 \\
1.805 \\
1.808 \\
1.808 \\
1.801 \\
1.814 \\
1.809 \\
1.809 \\
1.808 \\
\end{array}$ & $\begin{array}{l}1.38 \\
0.82 \\
1.12 \\
0.95 \\
1.00 \\
1.06 \\
1.00 \\
0.82 \\
0.90 \\
1.00 \\
1.06 \\
1.00 \\
0.95 \\
0.95 \\
1.00 \\
1.00 \\
1.12 \\
0.90 \\
0.95 \\
0.95 \\
\end{array}$ & $\begin{array}{l}0.020 \\
0.035 \\
0.015 \\
0.040 \\
0.005 \\
0.015 \\
0.040 \\
0.030 \\
0.030 \\
0.015 \\
0.030 \\
0.040 \\
0.030 \\
0.045 \\
0.035 \\
0.020 \\
0.020 \\
0.030 \\
0.035 \\
0.020 \\
\end{array}$ & $\begin{array}{l}0.190 \\
0.275 \\
0.290 \\
0.420 \\
0.355 \\
0.320 \\
0.260 \\
0.325 \\
0.250 \\
0.350 \\
0.320 \\
0.340 \\
0.310 \\
0.390 \\
0.390 \\
0.310 \\
0.240 \\
0.290 \\
0.290 \\
0.320 \\
\end{array}$ & $\begin{array}{l}1608 \\
1524 \\
2061 \\
2397 \\
2302 \\
1884 \\
1711 \\
1732 \\
1671 \\
2486 \\
2119 \\
2040 \\
1992 \\
2207 \\
2322 \\
2207 \\
1848 \\
1788 \\
1714 \\
2205 \\
\end{array}$ \\
\hline $\begin{array}{l}\text { Mean } \\
\text { Std. Dev. }\end{array}$ & 1 & $\begin{array}{l}1.808 \\
0.006\end{array}$ & $\begin{array}{l}1.00 \\
0.121\end{array}$ & $\begin{array}{l}0.027 \\
0.011\end{array}$ & $\begin{array}{l}0.312 \\
0.055\end{array}$ & $\begin{array}{r}1991 \\
285\end{array}$ \\
\hline
\end{tabular}


TABLE B-14 (Continued)

\begin{tabular}{|c|c|c|c|c|c|c|}
\hline $\begin{array}{c}\text { GA } \\
\text { Specimen } \\
\text { No. }\end{array}$ & $\begin{array}{l}\text { Location in } \\
\text { Log and } \\
\text { Orientation }\end{array}$ & $\begin{array}{l}\text { Density } \\
\left(\mathrm{g} / \mathrm{cm}^{3}\right)\end{array}$ & $\begin{array}{l}\text { Modulus of } \\
\text { Elasticity } \\
\times 10-6(b) \\
\quad \text { (psi) }\end{array}$ & $\begin{array}{c}\text { Permanent Set } \\
\text { After First } \\
\text { Loading } \\
(\%)\end{array}$ & $\begin{array}{c}\text { Strain at } \\
\text { Fracture } \\
(\%)\end{array}$ & $\begin{array}{l}\text { U1timate } \\
\text { Strength } \\
\quad \text { (psi) }\end{array}$ \\
\hline $\begin{array}{r}5651-73-3 \mathrm{~A}-006 \mathrm{~B} \\
-007 \mathrm{~B} \\
-012 \mathrm{~B} \\
-013 \mathrm{~B} \\
-018 \mathrm{~B} \\
-019 \mathrm{~B} \\
-024 \mathrm{~B} \\
-025 \mathrm{~B} \\
-027 \mathrm{~B} \\
-028 \mathrm{~B} \\
-3 \mathrm{~B}-006 \mathrm{~B} \\
-007 \mathrm{~B} \\
-012 \mathrm{~B} \\
-013 \mathrm{~B} \\
-018 \mathrm{~B} \\
-019 \mathrm{~B} \\
-024 \mathrm{~B} \\
-025 \mathrm{~B} \\
-027 \mathrm{~B} \\
-028 \mathrm{~B}\end{array}$ & MLC, axia1 & $\begin{array}{l}1.774 \\
1.767 \\
1.766 \\
1.776 \\
1.775 \\
1.765 \\
1.775 \\
1.776 \\
1.770 \\
1.777 \\
1.767 \\
1.765 \\
1.780 \\
1.767 \\
1.769 \\
1.778 \\
1.765 \\
1.766 \\
1.764 \\
1.763 \\
\end{array}$ & $\begin{array}{l}0.90 \\
0.95 \\
1.00 \\
0.90 \\
0.86 \\
0.69 \\
0.95 \\
1.00 \\
0.86 \\
0.90 \\
1.00 \\
1.00 \\
1.00 \\
0.95 \\
0.60 \\
0.86 \\
0.88 \\
0.90 \\
0.86 \\
0.86 \\
\end{array}$ & $\begin{array}{l}0.01 .5 \\
0.030 \\
0.025 \\
0.025 \\
0.020 \\
0.015 \\
0.010 \\
0.020 \\
0.010 \\
0.020 \\
0.017 \\
0.025 \\
0.010 \\
0.030 \\
0.030 \\
0.022 \\
0.020 \\
0.030 \\
0.015 \\
0.027 \\
\end{array}$ & $\begin{array}{l}0.210 \\
0.270 \\
0.310 \\
0.320 \\
0.200 \\
0.285 \\
0.275 \\
0.250 \\
0.245 \\
0.265 \\
0.323 \\
0.245 \\
0.185 \\
0.145 \\
0.240 \\
0.190 \\
0.215 \\
0.295 \\
0.275 \\
0.335\end{array}$ & $\begin{array}{l}1427 \\
1711 \\
2015 \\
1896 \\
1469 \\
1406 \\
1796 \\
1691 \\
1449 \\
1672 \\
1976 \\
1670 \\
1438 \\
1141 \\
1080 \\
1283 \\
1487 \\
1569 \\
1507 \\
1731 \\
\end{array}$ \\
\hline $\begin{array}{l}\text { Mean } \\
\text { Std. Dev. }\end{array}$ & $\gamma$ & $\begin{array}{l}1.770 \\
0.005\end{array}$ & $\begin{array}{l}0.90 \\
0.102\end{array}$ & $\begin{array}{l}0.021 \\
0.007\end{array}$ & $\begin{array}{l}0.254 \\
0.051\end{array}$ & $\begin{array}{r}1571 \\
252\end{array}$ \\
\hline $\begin{array}{r}5651-73-3 \mathrm{~A}-036 \mathrm{~B} \\
-037 \mathrm{~B} \\
-042 \mathrm{~B} \\
-043 \mathrm{~B} \\
-048 \mathrm{~B} \\
-049 \mathrm{~B} \\
-054 \mathrm{~B} \\
-055 \mathrm{~B} \\
-060 \mathrm{~A} \\
-060 \mathrm{~B} \\
-3 \mathrm{~B}-036 \mathrm{~B} \\
-037 \mathrm{~B} \\
-042 \mathrm{~B} \\
-043 \mathrm{~B} \\
-048 \mathrm{~B} \\
-049 \mathrm{~B} \\
-054 \mathrm{~B} \\
-055 \mathrm{~B} \\
-060 \mathrm{~A} \\
-060 \mathrm{~B}\end{array}$ & MLC, radial & $\begin{array}{l}1.766 \\
1.775 \\
1.781 \\
1.771 \\
1.781 \\
1.782 \\
1.778 \\
1.771 \\
1.767 \\
1.781 \\
1.776 \\
1.773 \\
1.779 \\
1.776 \\
1.785 \\
1.778 \\
1.777 \\
1.783 \\
1.770 \\
1.769 \\
\end{array}$ & $\begin{array}{l}- \\
0.95 \\
1.00 \\
0.78 \\
0.72 \\
1.00 \\
0.90 \\
0.95 \\
1.06 \\
0.90 \\
0.75 \\
(c) \\
0.95 \\
0.88 \\
0.90 \\
0.95 \\
1.00 \\
0.91 \\
0.83 \\
0.68 \\
\end{array}$ & $\begin{array}{l}- \\
0.025 \\
0.035 \\
0.030 \\
0.030 \\
0.020 \\
0.015 \\
0.020 \\
0.040 \\
0.010 \\
0.035 \\
(c) \\
0.030 \\
0.025 \\
0.030 \\
0.017 \\
0.047 \\
0.030 \\
0.025 \\
0.015 \\
\end{array}$ & $\begin{array}{l}0.140 \\
0.220 \\
0.205 \\
0.300 \\
0.245 \\
0.130 \\
0.210 \\
0.250 \\
0.350 \\
0.175 \\
0.330 \\
0.165 \\
0.210 \\
0.195 \\
0.252 \\
0.255 \\
0.205 \\
0.233 \\
0.295 \\
0.255 \\
\end{array}$ & $\begin{array}{r}957 \\
1428 \\
1367 \\
1266 \\
1388 \\
1120 \\
1450 \\
1531 \\
2400 \\
1170 \\
1448 \\
958 \\
1428 \\
1287 \\
1594 \\
1491 \\
1384 \\
1428 \\
1428 \\
1144 \\
\end{array}$ \\
\hline $\begin{array}{l}\text { Mean } \\
\text { Std. Dev. }\end{array}$ & 1 & $\begin{array}{l}1.776 \\
0.006\end{array}$ & $\begin{array}{l}0.90 \\
0.105\end{array}$ & $\begin{array}{l}0.027 \\
0.009\end{array}$ & $\begin{array}{l}0.231 \\
0.058\end{array}$ & $\begin{array}{r}1383 \\
298\end{array}$ \\
\hline
\end{tabular}

(a) Measured at UCC.

(b) Specimens were loaded to $1000 \mathrm{psi}$, unloaded to zero stress, and reloaded to failure while recording the stress-strain curve. Modulus of elasticity = chord modulus between 100 and 1000 psi on second loading.

(c) Specimen broke during first loading cycle. 
TABLE B-15

DENSITY AND TENSILE PROPERTIES OF TS-1240, LOT 1

[ $\log 5651-74$ (UCC 42), whole $\log$ density $=1.82 \mathrm{~g} / \mathrm{cm}^{3}{ }^{(a)}$, 0.505-in.-diameter by 3.00-in.-long specimens]

\begin{tabular}{|c|c|c|c|c|c|c|}
\hline $\begin{array}{c}\text { GA } \\
\text { Specimen } \\
\text { No. }\end{array}$ & $\begin{array}{l}\text { Location in } \\
\text { Log and } \\
\text { Orientation }\end{array}$ & $\begin{array}{l}\text { Density } \\
\left(\mathrm{g} / \mathrm{cm}^{3}\right)\end{array}$ & $\begin{array}{l}\text { Modulus of } \\
\text { Elasticity } \\
\times 10-6(b) \\
\quad(p s i)\end{array}$ & $\begin{array}{c}\text { Permanent Set } \\
\text { After First } \\
\text { Loading } \\
(\%)\end{array}$ & $\left\{\begin{array}{c}\text { Strain at } \\
\text { Fracture } \\
(\%)\end{array}\right.$ & $\begin{array}{l}\text { Ultimate } \\
\text { Strength } \\
\text { (psi) }\end{array}$ \\
\hline 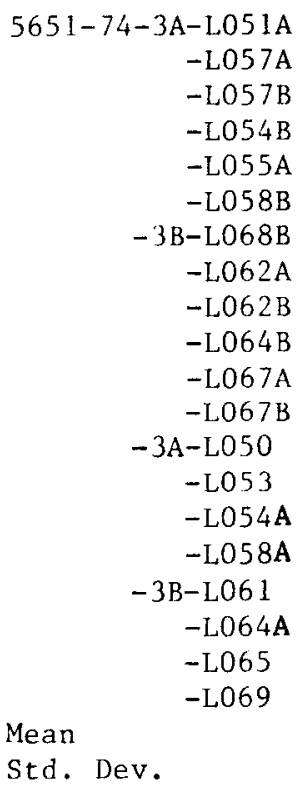 & 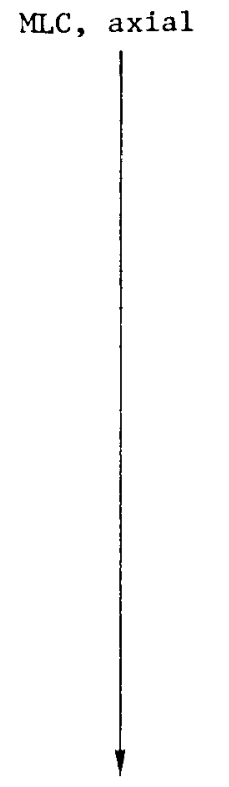 & $\begin{array}{l}1.797 \\
1.798 \\
1.795 \\
1.798 \\
1.793 \\
1.791 \\
1.784 \\
1.789 \\
1.793 \\
0.005\end{array}$ & $\begin{array}{l}1.10 \\
1.12 \\
1.08 \\
1.11 \\
1.06 \\
1.10 \\
1.05 \\
\frac{1.06}{1.08} \\
0.026\end{array}$ & $\begin{array}{l}0.018 \\
0.014 \\
0.021 \\
0.019 \\
0.020 \\
0.025 \\
0.024 \\
0.024 \\
0.021 \\
0.004\end{array}$ & $\begin{array}{l}0.306 \\
0.285 \\
0.275 \\
0.260 \\
0.221 \\
0.271 \\
0.286 \\
0.284 \\
0.273 \\
0.025\end{array}$ & $\begin{array}{l}2028 \\
1987 \\
2035 \\
2038 \\
1895 \\
2137 \\
2175 \\
1918 \\
2035 \\
2150 \\
1986 \\
1970 \\
2238 \\
2186 \\
2010 \\
1914 \\
1661 \\
1890 \\
1943 \\
1944 \\
2007 \\
131\end{array}$ \\
\hline $\begin{array}{r}5651-74-3 \text { A-L079 } \\
- \text { L081 } \\
- \text { L082 } \\
- \text { L084 } \\
-3 B-L 090 \\
-L 091 \\
-L 093 \\
-L 094 \\
- \text { L096 } \\
-3 A-L 075\end{array}$ & MLC, radial & 1.785 & 0.97 & 0.045 & 0.206 & $\begin{array}{l}1588 \\
1642 \\
1672 \\
1641 \\
2120 \\
2409 \\
1621 \\
1692 \\
1237 \\
1445\end{array}$ \\
\hline $\begin{array}{rr} & -L 076 \\
& \text { L077 } \\
& \text {-L080 } \\
-3 \text { B-L087 } & \text {-L088 } \\
& \text {-L089 } \\
& \text {-L092 }\end{array}$ & 1 & $\begin{array}{l}1.791 \\
1.790 \\
1.792 \\
1.791 \\
1.795 \\
1.793 \\
1.789 \\
1.791 \\
0.003\end{array}$ & $\begin{array}{l}1.02 \\
1.02 \\
1.03 \\
1.03 \\
1.05 \\
1.03 \\
1.01 \\
1.02 \\
0.023\end{array}$ & $\begin{array}{l}0.024 \\
0.026 \\
0.028 \\
0.026 \\
0.022 \\
0.022 \\
0.021 \\
0.027 \\
0.008\end{array}$ & $\begin{array}{l}0.250 \\
0.260 \\
0.265 \\
0.261 \\
0.335 \\
0.221 \\
0.189 \\
0.248 \\
0.045\end{array}$ & $\begin{array}{r}1692 \\
1749 \\
1505 \\
1835 \\
2284 \\
1630 \\
1372 \\
1714 \\
305\end{array}$ \\
\hline
\end{tabular}


TABLE B-15 (Continued)

\begin{tabular}{|c|c|c|c|c|c|c|}
\hline $\begin{array}{l}\text { GA } \\
\text { Specimen } \\
\text { No. }\end{array}$ & $\begin{array}{l}\text { Location in } \\
\text { Log and } \\
\text { Orientation }\end{array}$ & $\begin{array}{l}\text { Density } \\
\left(\mathrm{g} / \mathrm{cm}^{3}\right)\end{array}$ & $\begin{array}{l}\text { Modulus of } \\
\text { Elasticity } \\
x 10^{-6}(\mathrm{~b}) \\
\quad(p s i)\end{array}$ & $\begin{array}{c}\text { Permanent Set } \\
\text { After First } \\
\text { Loading } \\
(\%)\end{array}$ & $\begin{array}{c}\text { Strain at } \\
\text { Fracture } \\
(\%)\end{array}$ & $\begin{array}{l}\text { Ultimate } \\
\text { Strength } \\
\quad \text { (psi) }\end{array}$ \\
\hline 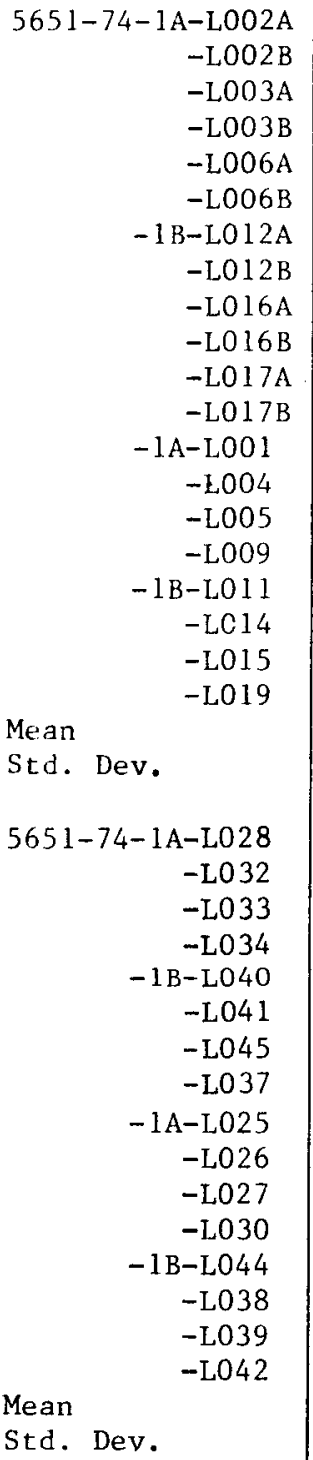 & EC, radia1 & $\begin{array}{l}1.800 \\
1.814 \\
1.802 \\
1.797 \\
1.807 \\
1.802 \\
1.798 \\
\frac{1.815}{1.804} \\
0.007\end{array}$ & $\begin{array}{l}1.15 \\
1.20 \\
1.18 \\
1.18 \\
1.23 \\
1.14 \\
1.15 \\
1.18 \\
1.18 \\
0.030\end{array}$ & $\begin{array}{l}0.018 \\
0.028 \\
0.013 \\
0.019 \\
0.025 \\
0.020 \\
0.023 \\
0.023 \\
0.021 \\
0.005\end{array}$ & $\begin{array}{l}0.250 \\
0.333 \\
0.310 \\
0.330 \\
0.295 \\
0.265 \\
0.320 \\
0.315 \\
0.302 \\
0.030\end{array}$ & $\begin{array}{r}2140 \\
2135 \\
2088 \\
2391 \\
2196 \\
1959 \\
2520 \\
2444 \\
2238 \\
1842 \\
2142 \\
2143 \\
1944 \\
2418 \\
2344 \\
2404 \\
2256 \\
1987 \\
2298 \\
2342 \\
2211 \\
187 \\
1633 \\
2077 \\
1818 \\
1942 \\
2171 \\
1992 \\
1932 \\
(c) \\
2462 \\
2145 \\
1948 \\
2980 \\
1946 \\
2534 \\
2369 \\
2843 \\
2186 \\
380\end{array}$ \\
\hline
\end{tabular}


TABLE B-15 (Continued)

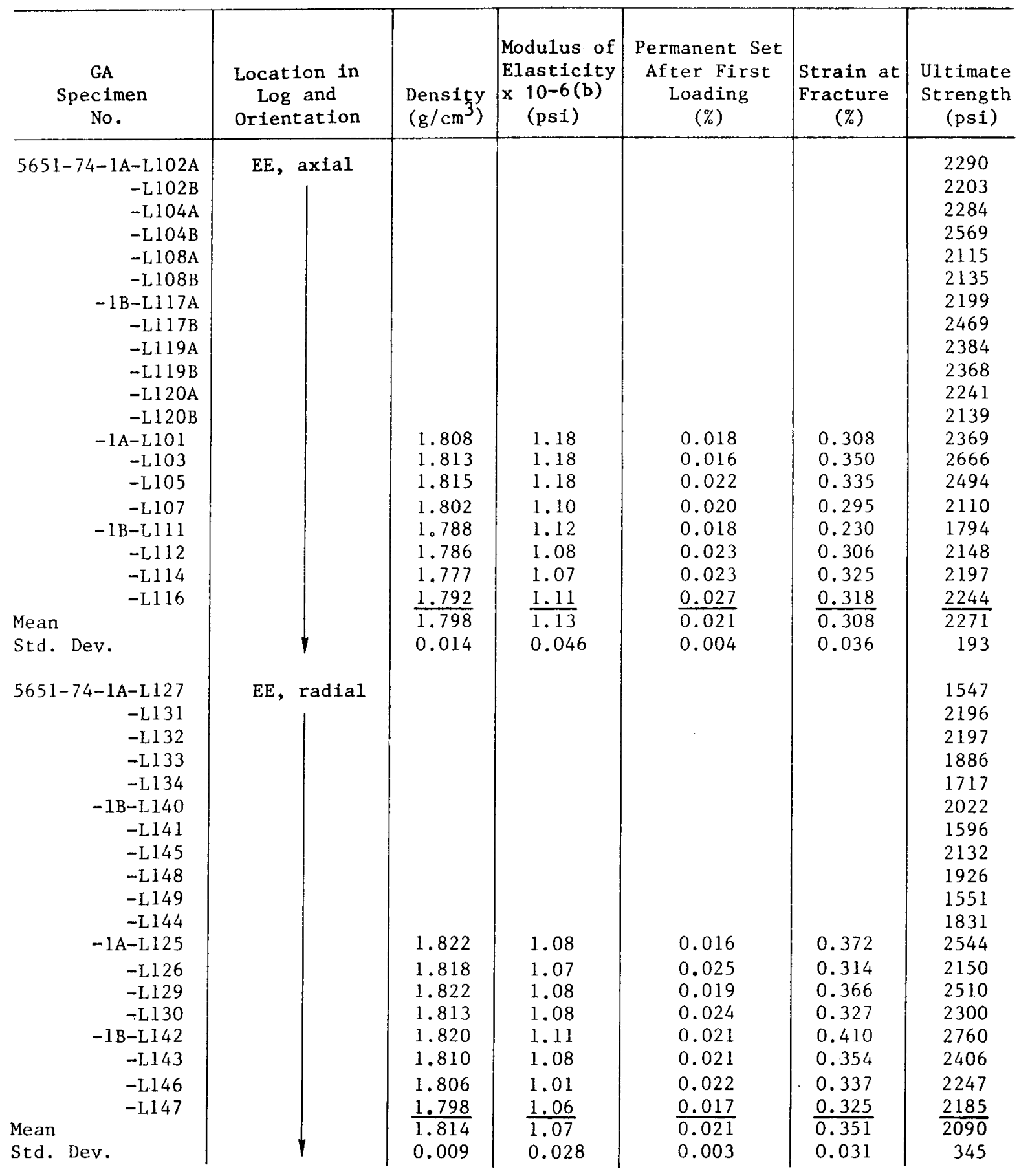


TABLE B-15 (Continued)

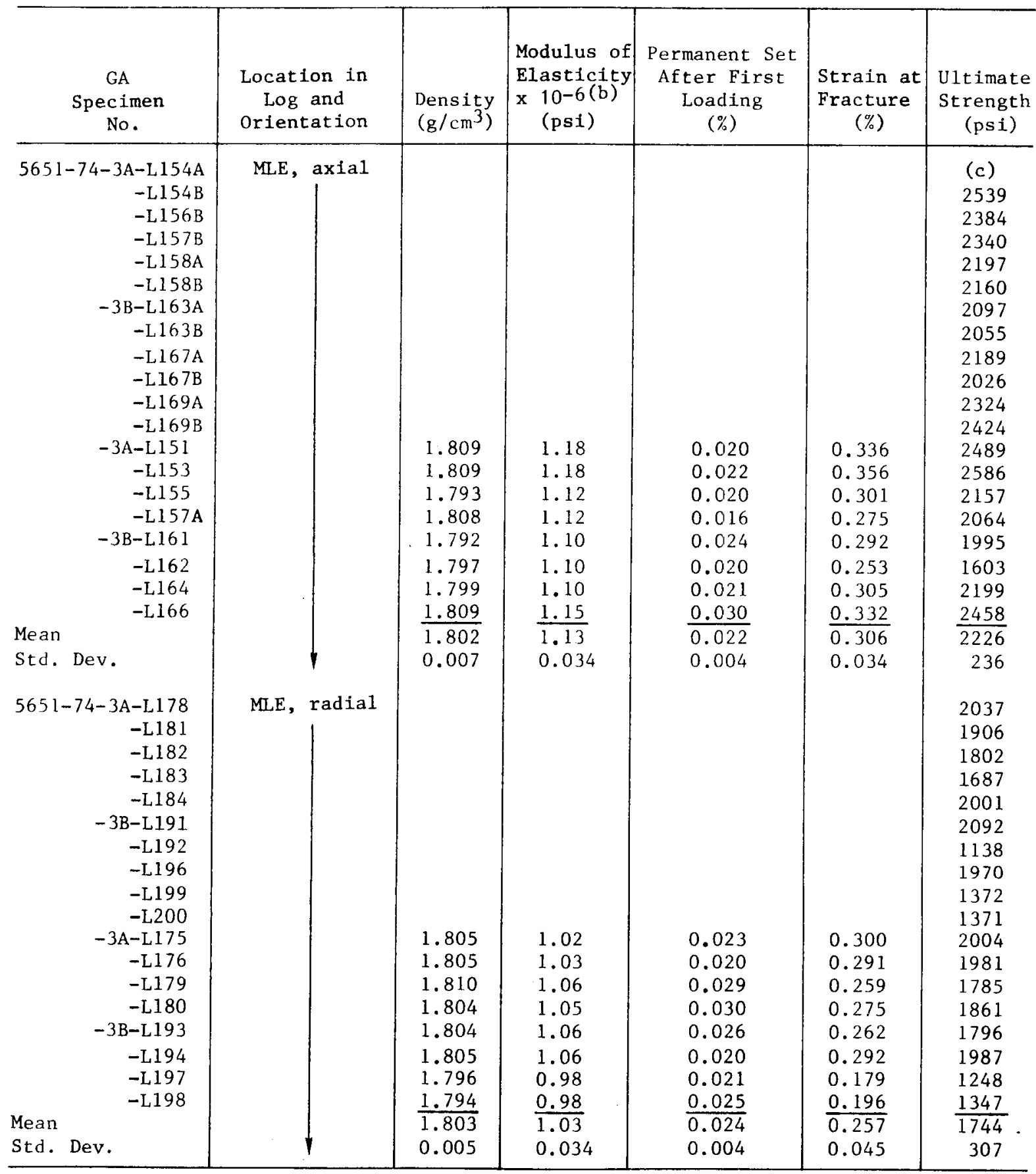

(a) Measured at UCC.

(b) Specimens were loaded to $1000 \mathrm{psi}$, unloaded to zero stress, and reloaded to failure while recording the stress-strain curve. Modulus of elasticity $=$ chord modulus between 100 and 1000 psi on second loading.

(c) Bond failure. 
TABLE B-16

DENSITY AND TENSILE PROPERTIES OF TS-1240, LOT

[Log 5651-75 (UCC 46), whole $\log$ density $=1.84 \mathrm{~g} / \mathrm{cm}^{3}{ }^{\text {(a) }}$, 0.505-in.-diameter by 3.00-in.-1ong specimens]

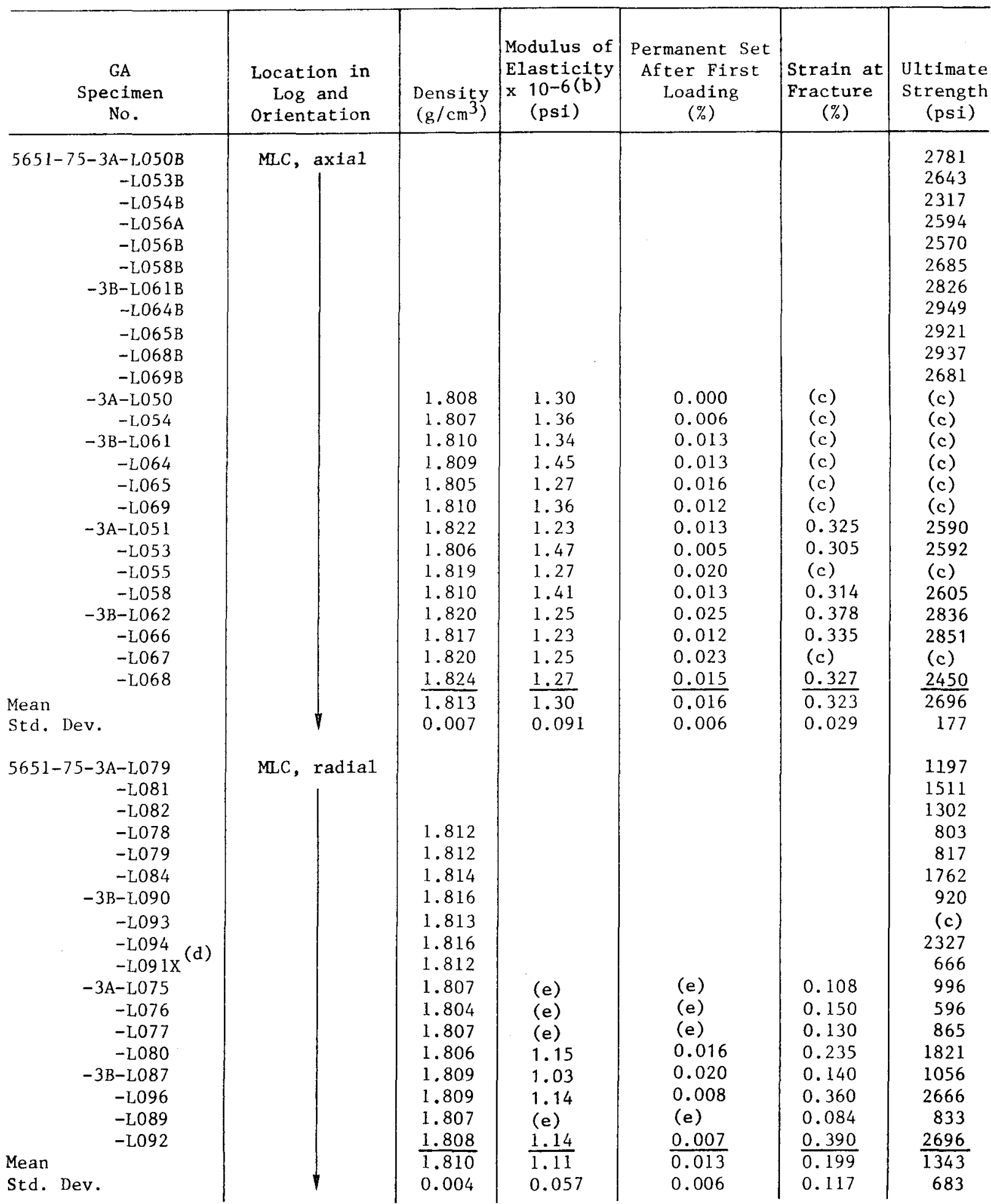


TABLE B-16 (Continued)

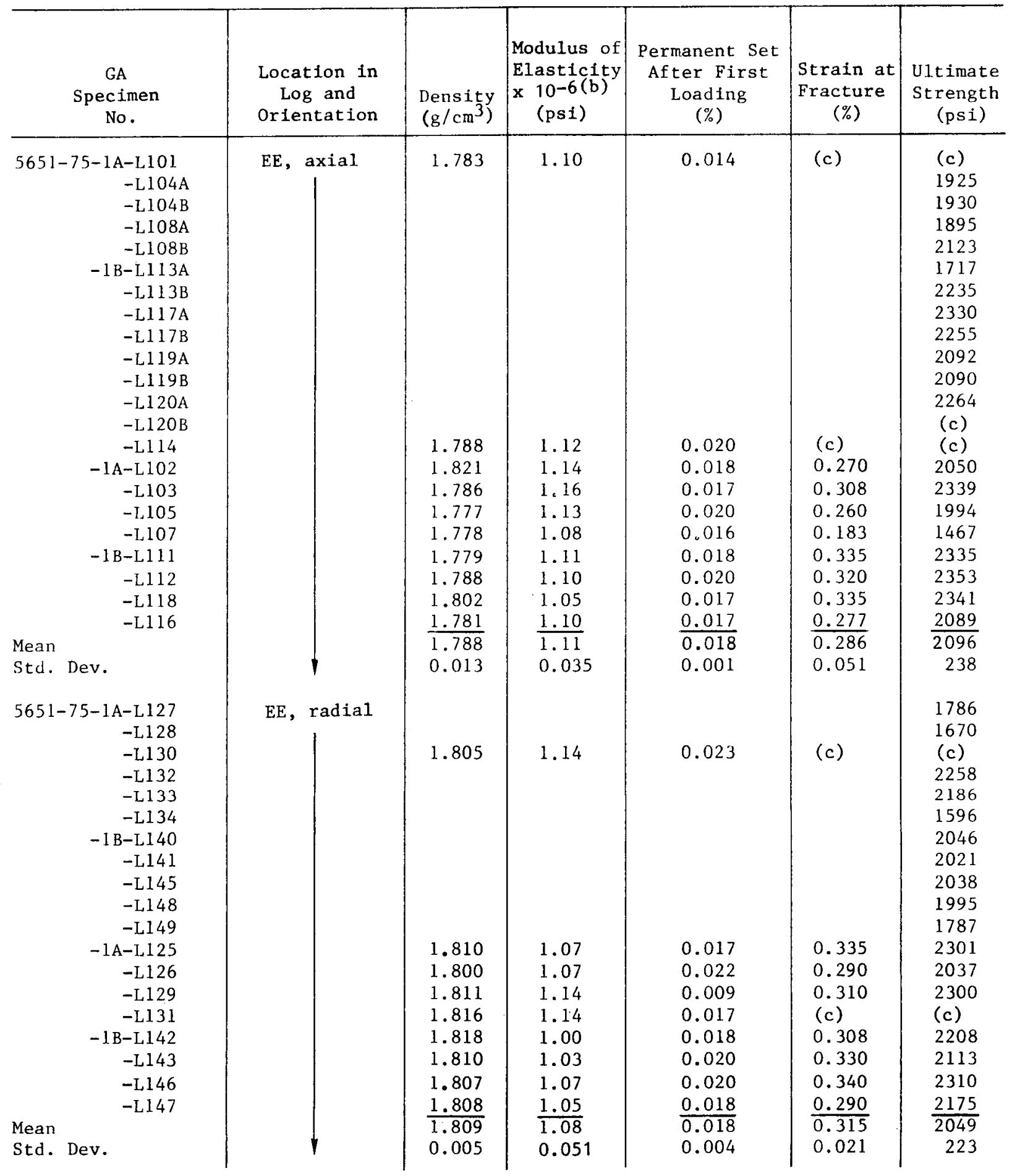


TABLE B-16 (Continued)

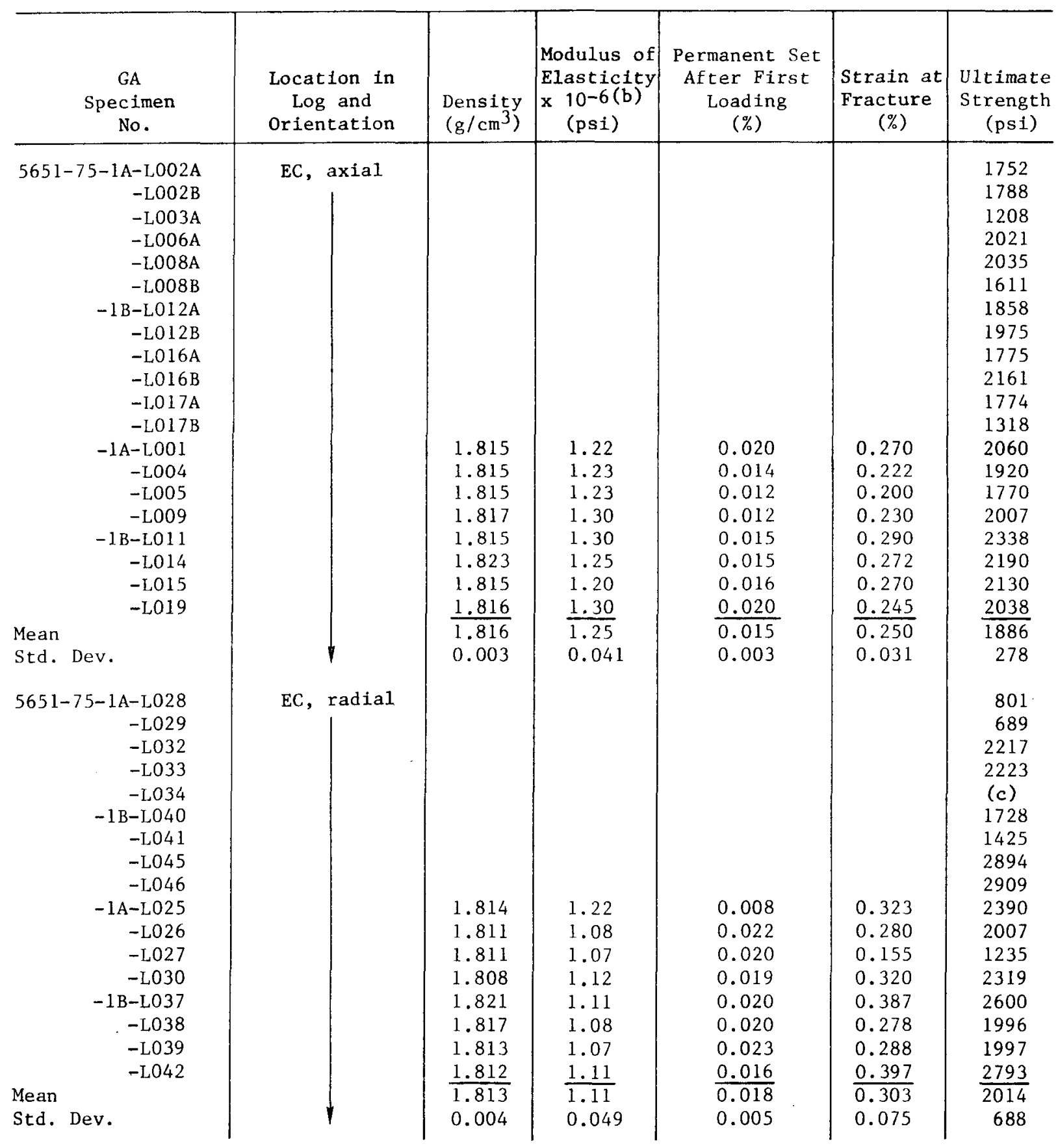


TABLE B-16 (Continued)

\begin{tabular}{|c|c|c|c|c|c|c|}
\hline $\begin{array}{c}\text { GA } \\
\text { Specimen } \\
\text { No. }\end{array}$ & $\begin{array}{l}\text { Location in } \\
\text { Log and } \\
\text { Orientation }\end{array}$ & $\begin{array}{l}\text { Density } \\
\left(\mathrm{g} / \mathrm{cm}^{3}\right)\end{array}$ & $\begin{array}{l}\text { Modulus of } \\
\text { Elasticity } \\
\times 10^{-6}(\mathrm{~b}) \\
\quad(p s i)\end{array}$ & $\begin{array}{l}\text { Permanent Set } \\
\text { After First } \\
\text { Loading } \\
(\%)\end{array}$ & $\begin{array}{c}\text { Strain at } \\
\text { Fracture } \\
(\%)\end{array}$ & $\begin{array}{l}\text { Ultimate } \\
\text { Strength } \\
\quad \text { (psi) }\end{array}$ \\
\hline $\begin{aligned} 5651-75-3 A-L 151 \\
-L 152 A \\
-L 152 B \\
-L 153 B \\
-L 154 A \\
-L 154 B \\
-L 156 B \\
-L 157 B \\
-3 B-L 163 A \\
-L 163 B \\
-L 170 A \\
-L 170 B \\
-3 A-L 155 \\
-L 157 A \\
-3 B-L 164 \\
-3 B-L 166 \\
-3 A-L 151 A \\
-L 153 \\
-L 158 \\
-L 160 \\
\text { Mean } \\
\text { Std. Dev. }\end{aligned}$ & $\left.\right|_{\text {MLE, radial }}$ & $\begin{array}{l}1.804 \\
1.804 \\
1.812 \\
1.815 \\
1.808 \\
1.807 \\
1.818 \\
1.827 \\
1.828 \\
1.826 \\
1.825 \\
1.826 \\
1.817 \\
0.009\end{array}$ & $\begin{array}{l}1.32 \\
1.34 \\
1.45 \\
1.32 \\
1.27 \\
1.34 \\
1.17 \\
1.22 \\
1.27 \\
1.29 \\
1.27 \\
1.27 \\
1.29 \\
0.07\end{array}$ & $\begin{array}{l}0.013 \\
0.014 \\
0.006 \\
0.010 \\
0.013 \\
0.015 \\
0.017 \\
0.019 \\
0.016 \\
0.015 \\
0.015 \\
0.009 \\
0.013 \\
0.004\end{array}$ & $\begin{array}{l}(c) \\
(c) \\
(c) \\
(c) \\
0.356 \\
0.340 \\
0.345 \\
0.360 \\
0.400 \\
0.365 \\
0.295 \\
0.295 \\
0.344 \\
0.035\end{array}$ & $\begin{array}{l}2824 \\
2689 \\
2623 \\
(c) \\
2743 \\
2399 \\
2240 \\
2688 \\
3012 \\
2794 \\
3032 \\
3037 \\
(c) \\
(c) \\
(c) \\
(c) \\
2828 \\
2703 \\
2597 \\
2752 \\
3152 \\
2928 \\
2569 \\
2578 \\
2741 \\
233 \\
1756 \\
1857 \\
1961 \\
1961 \\
2619 \\
1237 \\
2741 \\
1766 \\
1740 \\
1884 \\
1692 \\
1615 \\
1315 \\
1497 \\
2571 \\
2600 \\
1912 \\
471\end{array}$ \\
\hline
\end{tabular}

(a) Measured at UCC.

(b) Specimens were loaded to $1000 \mathrm{psi}$, unloaded to zero stress, and reloaded to failure while recording the stress-strain curve. Modulus of elasticity = chord modulus between 100 and 1000 psi on second loading.

(c) Bond failure.

(d) Specimen machined from extra core taken adjacent to core L091.

(e) Specimen broke during first loading cycle. 
TABLE $\quad B-17$

DENSITY AND TENSILE PROPERTIES OF TS-1240, Lot 1
$\left[\log 6484-29\right.$ (UCC $8-1 \mathrm{~S}-1 \mathrm{D}-23$ ) whole $\log$ density $=1.83 \mathrm{~g} / \mathrm{cm}^{3}$, 0.505-in.-diameter by 3.00-in.-1ong specimens]

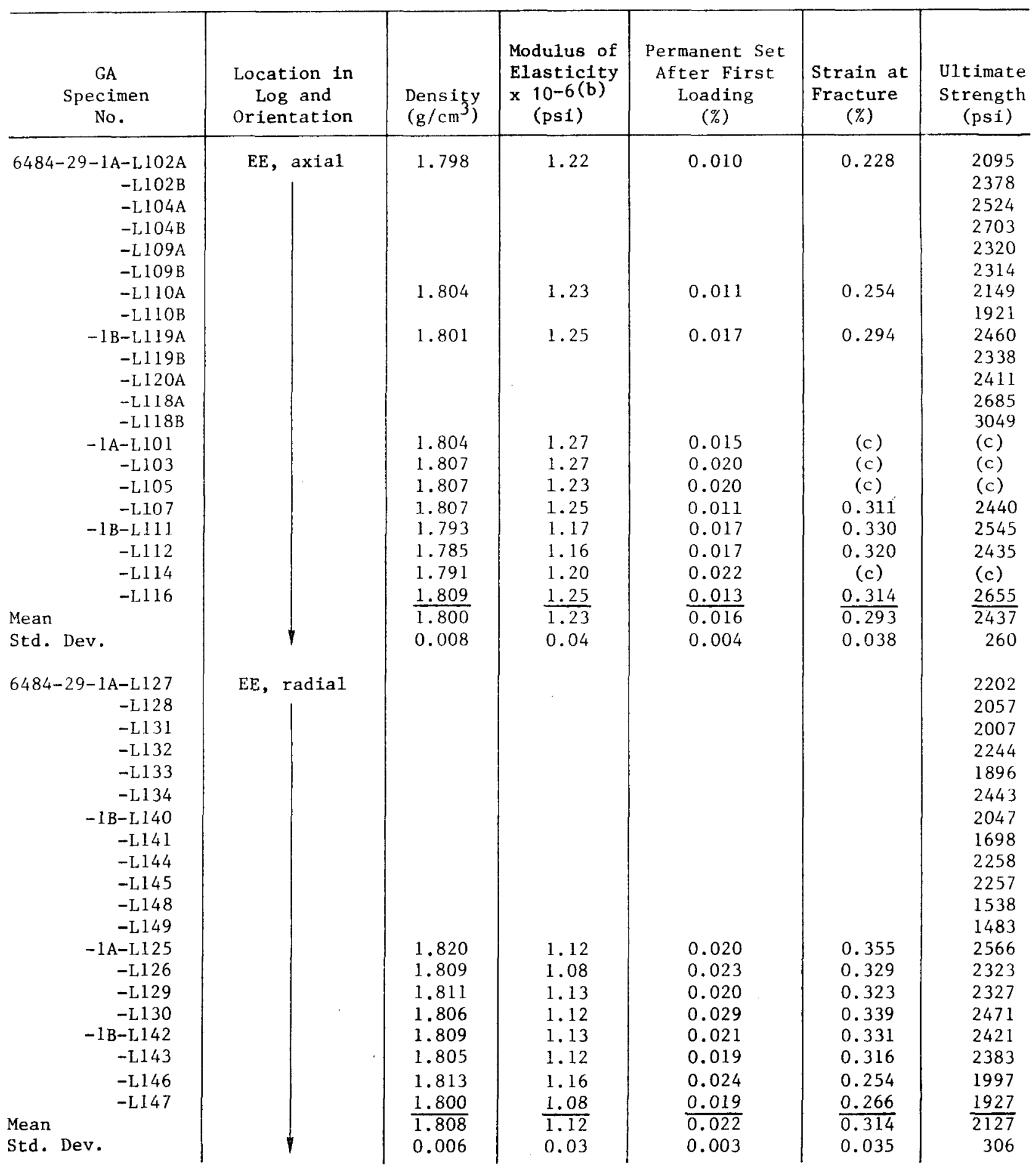


TABLE B-17 (Continued)

\begin{tabular}{|c|c|c|c|c|c|c|}
\hline $\begin{array}{c}\text { GA } \\
\text { Specimen } \\
\text { No. }\end{array}$ & $\begin{array}{l}\text { Location in } \\
\text { Log and } \\
\text { Orientation }\end{array}$ & $\begin{array}{l}\text { Density } \\
\left(\mathrm{g} / \mathrm{cm}^{3}\right)\end{array}$ & $\begin{array}{l}\text { Modulus of } \\
\text { Elasticity } \\
\times 10^{-6}(\mathrm{~b}) \\
\quad \text { (psi) }\end{array}$ & $\begin{array}{l}\text { Permanent Set } \\
\text { After First } \\
\text { Loading } \\
(\%)\end{array}$ & $\begin{array}{c}\text { Strain at } \\
\text { Fracture } \\
\qquad(\%)\end{array}$ & $\begin{array}{c}\text { Ultimate } \\
\text { Strength } \\
\text { (psi) }\end{array}$ \\
\hline 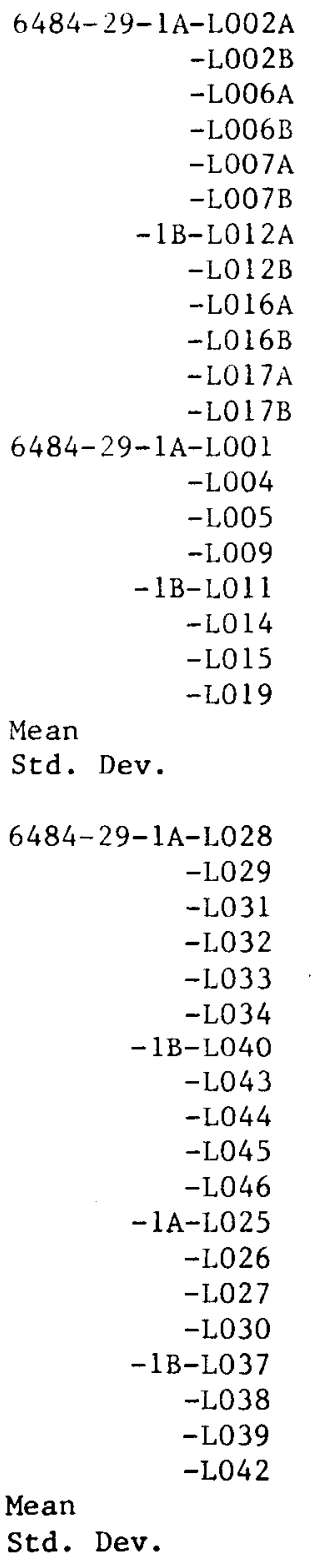 & $\mid$ & $\begin{array}{l}1.796 \\
1.802 \\
1.804 \\
1.799 \\
1.798 \\
1.794 \\
1.795 \\
1.802 \\
1.799 \\
0.004\end{array}$ & $\begin{array}{l}1.17 \\
1.22 \\
1.22 \\
1.19 \\
1.23 \\
1.27 \\
1.23 \\
1.23 \\
1.22 \\
0.03\end{array}$ & $\begin{array}{l}0.018 \\
0.020 \\
0.014 \\
0.015 \\
0.017 \\
0.021 \\
0.018 \\
0.019 \\
0.018 \\
0.002\end{array}$ & $\begin{array}{l}0.265 \\
0.314 \\
0.288 \\
0.299 \\
0.319 \\
0.325 \\
0.325 \\
0.304 \\
0.305 \\
0.021\end{array}$ & $\begin{array}{r}2186 \\
1989 \\
2220 \\
2421 \\
2264 \\
2557 \\
2177 \\
2045 \\
2358 \\
2491 \\
2486 \\
2133 \\
2041 \\
2527 \\
2381 \\
2441 \\
2565 \\
2585 \\
2603 \\
2425 \\
2345 \\
198 \\
\\
1254 \\
1553 \\
2238 \\
2032 \\
1653 \\
1597 \\
1018 \\
2551 \\
1771 \\
1962 \\
2057 \\
2500 \\
2191 \\
1808 \\
2596 \\
2575 \\
1887 \\
1875 \\
2007 \\
1954 \\
436\end{array}$ \\
\hline
\end{tabular}


TABLE B-17 (Continued)

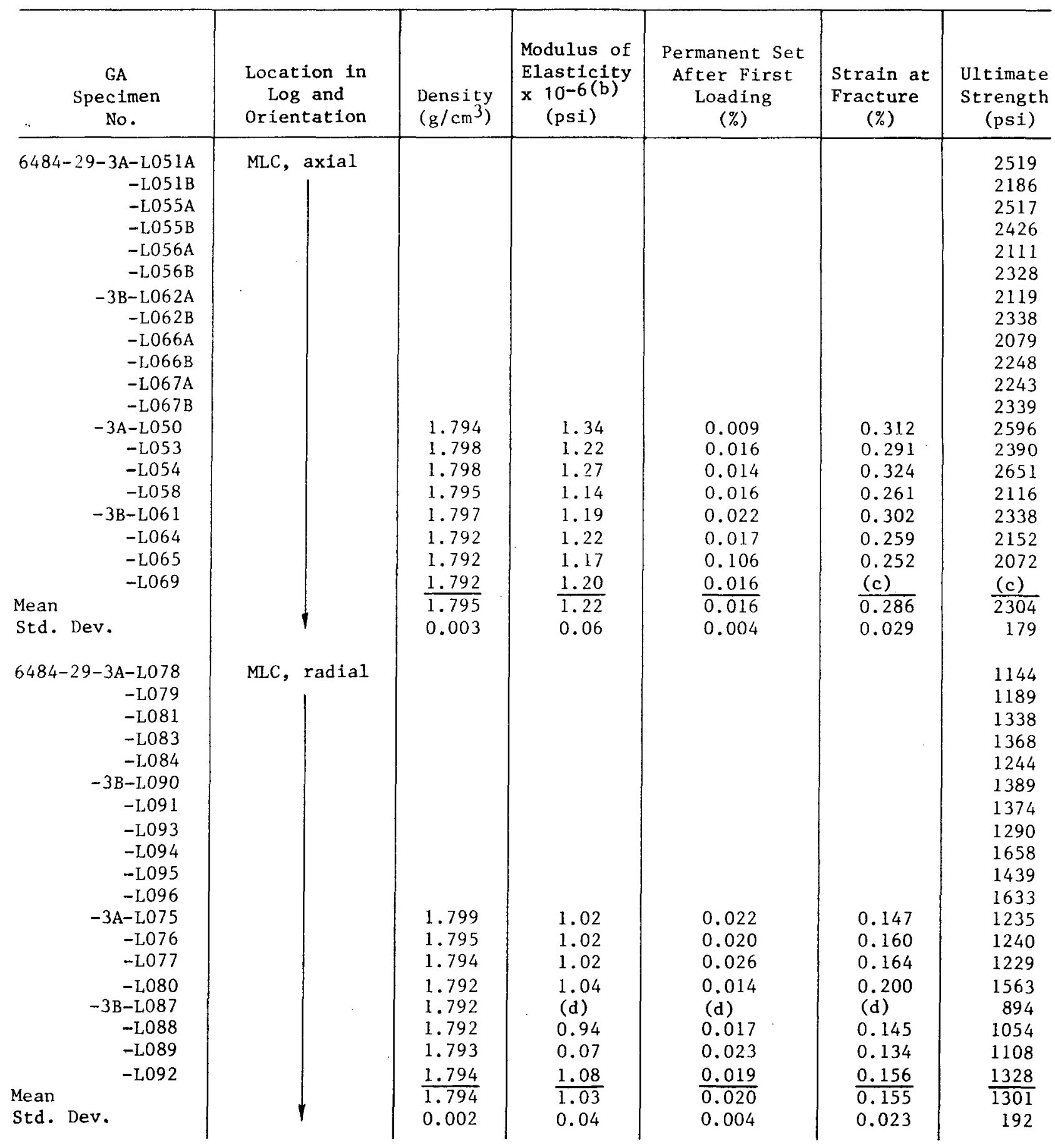


TABLE B-17 (Continued)

\begin{tabular}{|c|c|c|c|c|c|c|}
\hline $\begin{array}{c}\text { GA } \\
\text { Specimen } \\
\text { No. }\end{array}$ & $\begin{array}{l}\text { Location in } \\
\text { Log and } \\
\text { Orientation }\end{array}$ & $\begin{array}{l}\text { Density } \\
\left(\mathrm{g} / \mathrm{cm}^{3}\right)\end{array}$ & $\begin{array}{l}\text { Modulus of } \\
\text { Elasticity } \\
\times 10-6(b) \\
\quad \text { (psi) }\end{array}$ & $\begin{array}{c}\text { Permanent Set } \\
\text { Af ter: First } \\
\text { Loading } \\
(\%)\end{array}$ & $\begin{array}{c}\text { Strain at } \\
\text { Fracture } \\
(\%)\end{array}$ & $\begin{array}{l}\text { Ultimate } \\
\text { Strength } \\
\text { (psi) }\end{array}$ \\
\hline 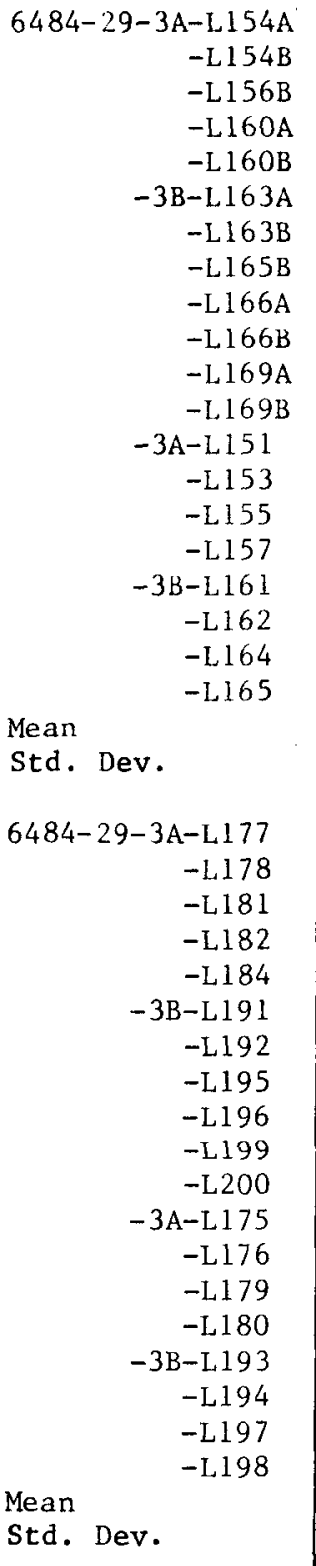 & MLE, radial & $\begin{array}{l}1.804 \\
1.803 \\
1.807 \\
1.805 \\
1.800 \\
1.792 \\
1.797 \\
1.797 \\
1.800 \\
0.005\end{array}$ & $\begin{array}{l}1.27 \\
1.34 \\
1.32 \\
1.23 \\
1.22 \\
1.20 \\
1.27 \\
1.23 \\
1.25 \\
0.05\end{array}$ & $\begin{array}{l}0.013 \\
0.015 \\
\\
\\
0.021 \\
0.016 \\
0.019 \\
0.018 \\
0.018 \\
0.014 \\
0.020 \\
0.014 \\
0.017 \\
0.003\end{array}$ & $\begin{array}{l}0.320 \\
\\
0.223 \\
\\
\\
\\
0.335 \\
(c) \\
(c) \\
0.354 \\
0.365 \\
0.302 \\
0.336 \\
0.316 \\
0.319 \\
0.044 \\
\end{array}$ & $\begin{array}{l}(c) \\
2742 \\
2665 \\
2733 \\
2582 \\
2489 \\
1863 \\
1995 \\
2700 \\
2837 \\
2490 \\
(c) \\
2743 \\
(c) \\
(c) \\
2753 \\
2804 \\
2467 \\
2665 \\
2605 \\
2571 \\
275 \\
1349 \\
1419 \\
1649 \\
1732 \\
1104 \\
1507 \\
1018 \\
1408 \\
1437 \\
948 \\
1013 \\
1549 \\
1758 \\
1844 \\
1724 \\
1543 \\
1394 \\
1023 \\
998 \\
1390 \\
293\end{array}$ \\
\hline
\end{tabular}

(a) Measured at UCC.

(b) Specimens were loaded to $1000 \mathrm{psi}$, unloaded to zero stress and reloaded to failure while recording the stress-strain curve. Modulus of elasticity = chord modulus between 100 and 1000 psi on second loading.

(c) Bond failure.

(d) Specimen broke during first loading cycle. 


\begin{tabular}{|c|c|c|c|c|c|c|c|c|c|c|c|c|c|c|c|}
\hline \multirow{2}{*}{$\begin{array}{l}\text { GA Specimen No./Log No. } \\
\text { (GLCC Log No./Lot No.) }\end{array}$} & \multirow{2}{*}{$\begin{array}{l}\text { Specimen } \\
\text { size } \\
\text { (in.) }\end{array}$} & \multirow{2}{*}{$\begin{array}{c}\text { No. of } \\
\text { Biaxial } \\
\text { Gages }\end{array}$} & \multirow{2}{*}{$\begin{array}{c}\text { Location } \\
\text { in Log }\end{array}$} & \multirow{2}{*}{$\begin{array}{l}\text { Direction } \\
\text { of Stress }\end{array}$} & \multirow{2}{*}{$\begin{array}{c}\text { Direction } \\
\text { of Transverse Strain } \\
\text { (Poisson's Ratio } \\
\text { Tensor Designation) }\end{array}$} & \multirow[b]{2}{*}{ Loading } & \multicolumn{9}{|c|}{ Poisson's Rat1o (Standard Deviation) } \\
\hline & & & & & & & $200 \mathrm{psi}$ & $250 \mathrm{psi}$ & $400 \mathrm{psi}$ & $500 \mathrm{ps} 1$ & $600 \mathrm{ps}$ & $750 \mathrm{psi}$ & $800 \mathrm{psi}$ & $1000 \mathrm{psi}$ & $1200 \mathrm{psi}$ \\
\hline $\begin{array}{l}5947-102-1 / \\
5651-63-1 \\
(22 / 266)\end{array}$ & $\begin{array}{l}0.5 \times 0.5 \\
\times 6 \text { long }\end{array}$ & $2\left(90^{\circ}\right.$ apart $)$ & MLC & Radial & $\begin{array}{l}\text { Axtal } \\
\left(v_{13}=v_{23}\right) \\
\text { Radial } \\
\left(v_{12}=v_{21}\right)\end{array}$ & \begin{tabular}{|c}
1 \\
2 \\
3 \\
Mean \\
1 \\
2 \\
3 \\
Mean
\end{tabular} & $\begin{array}{l}0.125 \\
0.109 \\
\\
0.140 \\
0.119\end{array}$ & & $\begin{array}{l}0.121 \\
\frac{0.119}{0.119} \\
0.139 \\
\frac{0.138}{0.138}\end{array}$ & & $\begin{array}{l}0.116 \\
0.131\end{array}$ & & $\begin{array}{l}0.112 \\
0.117 \\
0.121 \\
0.121 \\
0.127 \\
0.134 \\
0.136 \\
0.136\end{array}$ & 0.108 & $\begin{array}{l}0.105 \\
0.115 \\
0.119 \\
0.119 \\
0.118 \\
0.134 \\
\frac{0.136}{0.136}\end{array}$ \\
\hline $\begin{array}{l}5947-102-2 / \\
5651-63 \\
(22 / 266)\end{array}$ & $\begin{array}{l}0.5 \times 0.5 \\
\times 6 \text { long }\end{array}$ & $4\left(90^{\circ}\right.$ apart $)$ & MLC & Radial & $\begin{array}{l}\text { Axda1 } \\
\left(v_{13}=v_{23}\right) \\
\text { Radial } \\
\left(v_{12}=v_{21}\right)\end{array}$ & $\begin{array}{c}1 \\
2 \\
3 \\
4 \\
5 \\
6 \\
7 \\
8 \\
9 \\
10 \\
\text { Mean } \\
1 \\
2 \\
3 \\
4 \\
5 \\
6 \\
7 \\
8 \\
9 \\
10 \\
\text { Mean }\end{array}$ & $\begin{array}{ll}0.089 & (0.027) \\
0.066 & (0.015) \\
0.086 & (0.024) \\
& \\
0.102 & (0.021) \\
& \\
0.094 & (0.021) \\
0.098 & (0.014) \\
0.067 & (0.004) \\
0.095 & (0.011) \\
& \\
0.113 & (0.013) \\
& \\
0.104 & (0.014)\end{array}$ & & $\begin{array}{l}0.096(0.032) \\
0.096(0.023) \\
0.096(0.024) \\
0.100(0.027) \\
0.098(0.021) \\
0.102(0.013) \\
0.105(0.018) \\
0.106(0.013) \\
0.102(0.001) \\
0.104(0.010)\end{array}$ & & $\begin{array}{l}0.093(0.028) \\
\\
0.096(0.026) \\
\frac{0.095(0.027)}{0.095(0.027)} \\
\\
\\
0.098(0.010) \\
0.104(0.011) \\
\frac{0.103(0.011)}{0.103(0.011)}\end{array}$ & & 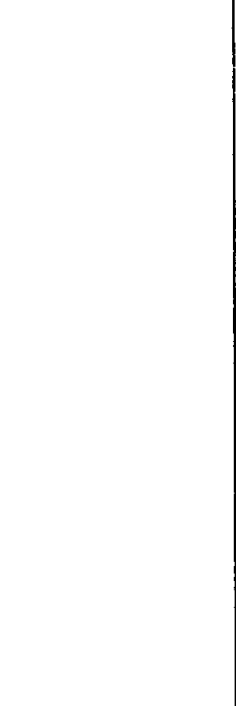 & . & \\
\hline $\begin{array}{l}5947-102-4 / \\
5651-63 \\
(22 / 266)\end{array}$ & $\begin{array}{r}0.5 \times 0.5 \\
\times 6 \text { long }\end{array}$ & $2\left(90^{\circ}\right.$ apart $)$ & MLC & Axtal & $\stackrel{\operatorname{Radial}}{\left(v_{31}=v_{32}\right)}$ & $\begin{array}{r}1 \\
2 \\
3 \\
\text { Mean }\end{array}$ & $\mid \begin{array}{ll}0.137 & (0.004) \\
0.134 & (0.008)\end{array}$ & & $\begin{array}{l}0.131(0.007) \\
\frac{0.124(0.013)}{0.124(0.013)}\end{array}$ & & $0.128(0.008)$ & & $\begin{array}{l}0.124(0.010) \\
0.130(0.011) \\
0.126(0.011) \\
0.126(0.011)\end{array}$ & $0.119(0.010)$ & \begin{tabular}{|l}
$0.114(0.011)$ \\
$0.126(0.011)$ \\
$0.126(0.011)$ \\
$0.126(0.011)$
\end{tabular} \\
\hline $\begin{array}{l}5947-5 / \\
5651-28 \\
(18 / 266)\end{array}$ & $\begin{array}{l}0.505 \text { diameter } \\
\times 6 \text { long }\end{array}$ & $3\left(120^{\circ}\right.$ apart $)$ & MLC & Axial & $\begin{array}{l}\text { Radial } \\
\left(v_{31}=v_{32}\right)\end{array}$ & $\begin{array}{c}1 \\
2 \\
3 \\
4 \\
5 \\
6 \\
\text { Mean }\end{array}$ & & $\begin{array}{l}0.149(0.052) \\
0.133(0.023) \\
0.136(0.018) \\
0.134(0.019)\end{array}$ & & $\left|\begin{array}{ll}0.131 & (0.017) \\
0.130 & (0.013) \\
0.131 & (0.019) \\
& \\
0.130 & (0.015)\end{array}\right|$ & & $\begin{array}{l}0.127(0.008) \\
0.126(0.014) \\
0.126(0.010) \\
0.129(0.020) \\
0.126(0.027) \\
0.125(0.021) \\
0.127(0.022)\end{array}$ & & & \\
\hline
\end{tabular}


TABLE B-18 (Continued)

\begin{tabular}{|c|c|c|c|c|c|c|c|c|c|c|c|c|c|c|c|}
\hline \multirow{2}{*}{$\begin{array}{l}\text { GA Specimen No./Log No. } \\
\text { (GLCC Log No./Lot No.) }\end{array}$} & \multirow{2}{*}{$\begin{array}{l}\text { Specimen } \\
\text { Size } \\
\text { (in.) }\end{array}$} & \multirow{2}{*}{$\begin{array}{l}\text { No. of } \\
\text { Biaxia1 } \\
\text { Gages }\end{array}$} & \multirow{2}{*}{$\begin{array}{l}\text { Location } \\
\text { in Log }\end{array}$} & \multirow{2}{*}{$\begin{array}{l}\text { Direction } \\
\text { of Stress }\end{array}$} & \multirow{2}{*}{$\begin{array}{c}\text { Direction } \\
\text { of Transverse Strain } \\
\text { (Poisson's Ratio } \\
\text { Tensor Designation) }\end{array}$} & \multirow[b]{2}{*}{ Loading } & \multicolumn{9}{|c|}{ Poisson's Ratio (Standard Deviation) } \\
\hline & & & & & & & $200 \mathrm{psi}$ & $250 \mathrm{psi}$ & $400 \mathrm{psi}$ & $500 \mathrm{psi}$ & $600 \mathrm{ps} 1$ & $750 \mathrm{ps} 1$ & $800 \mathrm{psi}$ & $1000 \mathrm{psi}$ & $1200 \mathrm{psi}$ \\
\hline $\begin{array}{l}5651-28-133 / \\
5651-28 \\
(18 / 266)\end{array}$ & $\begin{array}{l}0.5 \times 0.5 \\
\times 6 \text { long }\end{array}$ & $4\left(90^{\circ}\right.$ apart $)$ & MLE & Axial & $\begin{array}{l}\text { Radial } \\
\left(v_{32}\right) \\
\\
\text { Perpendicular } \\
\text { to radius in } \\
\text { radial plane } \\
\left(v_{31}\right)\end{array}$ & $\begin{array}{r}6 \\
7 \\
8 \\
12 \\
12 \\
13 \\
\text { Mean } \\
6 \\
7 \\
8 \\
12 \\
13 \\
13 \\
\text { Mean }\end{array}$ & & & $\begin{array}{l}0.124(0.009) \\
0.124(0.010) \\
\\
0.124(0.008) \\
0.117(0.006) \\
0.117(0.006) \\
\\
0.117(0.005)\end{array}$ & & $\begin{array}{l}0.122(0.012) \\
0.126(0.011) \\
\\
0.124(0.010) \\
\\
0.115(0.006) \\
0.115(0.004) \\
\\
0.115(0.004)\end{array}$ & & $\begin{array}{c}0.121(0.016) \\
0.121(0.016) \\
0.121(0.013) \\
\\
\\
0.106(0.007) \\
\left.\frac{0.106}{0.0099}\right) \\
0.106(0.007)\end{array}$ & & \\
\hline $\begin{array}{l}6399-8-\mathrm{C} / \\
5651-63 / \\
(22 / 266)\end{array}$ & $\begin{array}{l}0.5 \times 0.5 \\
\times 6 \text { long }\end{array}$ & $4\left(90^{\circ}\right.$ apart $)$ & MLE & Axial & $\begin{array}{l}\text { Perpendicular } \\
\text { to radius in } \\
\text { radial plane } \\
\left(v_{31}\right) \\
\begin{array}{l}\text { Radial } \\
\left(v_{32}\right)\end{array}\end{array}$ & $\begin{array}{c}1 \\
2 \\
3 \\
4 \\
5 \\
6 \\
7 \\
8 \\
9 \\
10 \\
\text { Mean } \\
1 \\
2 \\
3 \\
4 \\
5 \\
6 \\
7 \\
8 \\
9 \\
10 \\
\text { Mean }\end{array}$ & 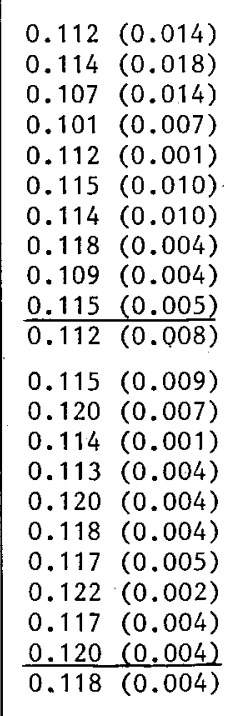 & & 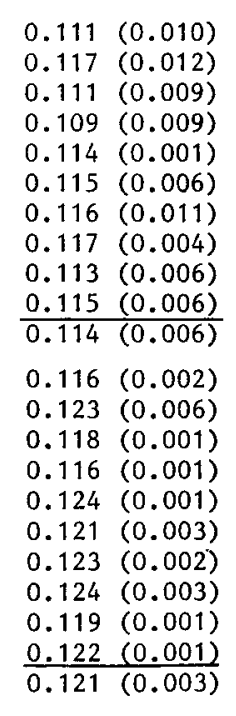 & & 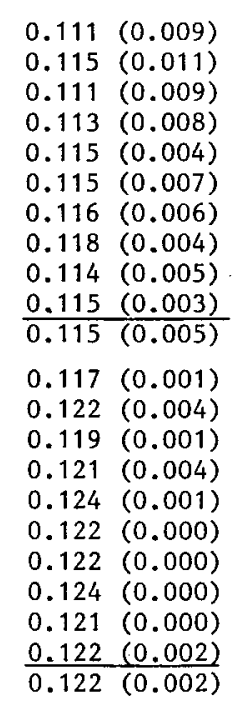 & & $\begin{array}{l}0.108(0.006) \\
0.116(0.004) \\
0.112(0.007) \\
0.112(0.007) \\
0.114(0.007) \\
0.115(0.004) \\
0.116(0.006) \\
0.115(0.006) \\
0.118(0.004) \\
0.115(0.005) \\
0.115(0.005) \\
0.115(0.005) \\
0.115(0.005) \\
0.115(0.001) \\
0.123(0.003) \\
0.120(0.001) \\
0.122(0.001) \\
0.123(0.001) \\
0.123(0.001) \\
0.123(0.001) \\
0.124(0.001) \\
0.122(0.001) \\
0.12(0.001) \\
0.122(0.001) \\
0.123(0.001)\end{array}$ & & \\
\hline
\end{tabular}


TABLE B-18 (Continued)

\begin{tabular}{|c|c|c|c|c|c|c|c|c|c|c|c|c|c|c|c|}
\hline \multirow{2}{*}{$\begin{array}{l}\text { GA Specimen No./Log No. } \\
\text { (GLCC Log No./Lot No.) }\end{array}$} & \multirow{2}{*}{$\begin{array}{l}\text { Specimen } \\
\text { Size } \\
\text { (in.) }\end{array}$} & \multirow{2}{*}{$\begin{array}{l}\text { No. of } \\
\text { Biaxia1 } \\
\text { Gages }\end{array}$} & \multirow{2}{*}{$\begin{array}{l}\text { Location } \\
\text { in Log }\end{array}$} & \multirow{2}{*}{$\begin{array}{l}\text { Direction } \\
\text { of Stress }\end{array}$} & \multirow{2}{*}{$\begin{array}{c}\text { Direction } \\
\text { of Transverse Strain } \\
\text { (poisson's Ratio } \\
\text { Tensor Designation) }\end{array}$} & \multirow[b]{2}{*}{ Loading } & \multicolumn{9}{|c|}{ Poisson's Rat1o (Standard Deviation) } \\
\hline & & & & & & & $200 \mathrm{psi}$ & $250 \mathrm{psi}$ & $400 \mathrm{psi}$ & $500 \mathrm{psi}$ & $600 \mathrm{psi}$ & $750 \mathrm{psi}$ & $800 \mathrm{psi}$ & $1000 \mathrm{psi}$ & $1200 \mathrm{psi}$ \\
\hline $\begin{array}{l}6399-8-\mathrm{D} / \\
5651-63 \\
(22 / 266)\end{array}$ & $\begin{array}{l}0.5 \times 0.5 \\
\times 6 \text { long }\end{array}$ & $4\left(90^{\circ}\right.$ apart $)$ & MLC & Radia1 & $\begin{array}{l}\left.\operatorname{Radia1}_{12}=v_{21}\right) \\
\text { Axial } \\
\left(v_{13}=v_{23}\right)\end{array}$ & $\begin{array}{c}1 \\
2 \\
3 \\
4 \\
5 \\
6 \\
7 \\
8 \\
9 \\
10 \\
\text { Mean } \\
1 \\
2 \\
3 \\
4 \\
5 \\
6 \\
7 \\
8 \\
9 \\
10 \\
\text { Mean }\end{array}$ & 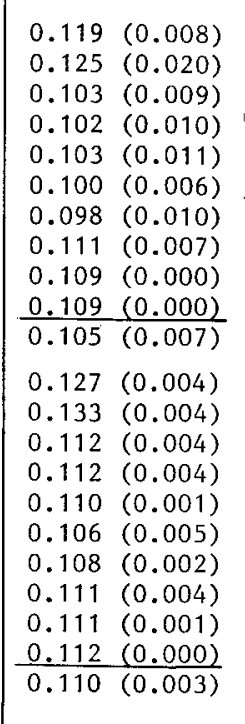 & & 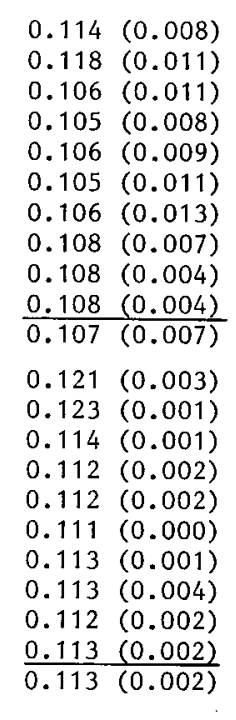 & & 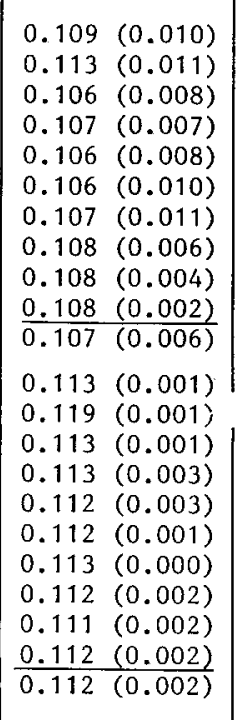 & $\begin{array}{l}1 \\
1 \\
1\end{array}$ & 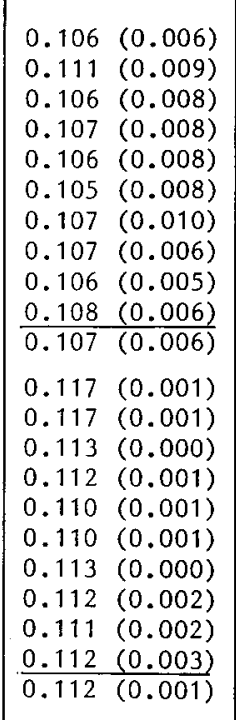 & & \\
\hline $\begin{array}{l}6399-8-\mathrm{A} / \\
5651-63 \\
(22 / 266)\end{array}$ & $\begin{array}{l}0.5 \times 0.5 \\
\times 6 \text { long }\end{array}$ & $4\left(90^{\circ}\right.$ apart $)$ & EE & Axial & $\begin{array}{l}\text { Perpendicular } \\
\text { to radius in } \\
\text { radia1 plane } \\
\left(v_{31}\right) \\
\\
\text { Radial } \\
\left(v_{32}\right)\end{array}$ & $\begin{array}{c}1 \\
2 \\
3 \\
4 \\
5 \\
6 \\
7 \\
7 \\
\text { Mean } \\
1 \\
2 \\
3 \\
4 \\
5 \\
6 \\
7 \\
\text { Mean }\end{array}$ & 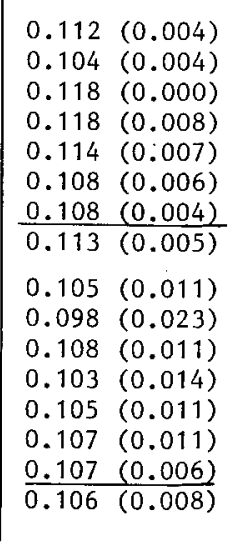 & & $\begin{array}{l}0.112(0.005) \\
0.112(0.007) \\
0.11(0.006) \\
0.121(0.0012) \\
0.116(0.012) \\
0.117(0.012) \\
0.17(0.006) \\
0.117(0.006) \\
0.118(0.007) \\
0.182(0.007) \\
0.106(0.020) \\
0.108(0.016) \\
0.108(0.017) \\
0.107(0.015) \\
0.112(0.013) \\
0.111(0.013) \\
0.111(0.013) \\
0.109(0.011)\end{array}$ & & $\begin{array}{l}0.111(0.008) \\
0.113(0.012) \\
0.119(0.008) \\
0.121(0.011) \\
0.117(0.008) \\
0.118(0.007) \\
0.118(0.007) \\
0.119(0.006) \\
0.103(0.008) \\
0.106(0.017) \\
0.111(0.014) \\
0.111(0.014) \\
0.110(0.013) \\
0.112(0.014) \\
0.113(0.013) \\
0.112(0.010)\end{array}$ & & $\begin{array}{ll}0.111 & (0.015) \\
0.119 & (0.008) \\
0.119 & (0.011) \\
0.1177 & (0.009) \\
0.119 & (0.007) \\
0.1120 & (0.007) \\
0.120 & (0.007) \\
0.119 & (0.006) \\
0.106 & (0.014) \\
0.110 & (0.015) \\
0.1111 & (0.016) \\
0.110 & (0.017) \\
0.1111 & (0.016) \\
0.1144 & (0.014) \\
0.111 & (0.011)\end{array}$ & & \\
\hline
\end{tabular}


TABLE B-18 (Continued)

\begin{tabular}{|c|c|c|c|c|c|c|c|c|c|c|c|c|c|c|c|}
\hline \multirow{2}{*}{$\begin{array}{l}\text { GA Specimen No./Log No. } \\
\text { (GLCC Log No./Lot No.) }\end{array}$} & \multirow{2}{*}{$\begin{array}{l}\text { Specimen } \\
\text { Size } \\
\text { (in.) }\end{array}$} & \multirow{2}{*}{$\begin{array}{l}\text { No. of } \\
\text { Biaxial } \\
\text { Gages }\end{array}$} & \multirow{2}{*}{$\begin{array}{l}\text { Location } \\
\text { in Log }\end{array}$} & \multirow{2}{*}{$\begin{array}{l}\text { Direction } \\
\text { of Stress }\end{array}$} & \multirow{2}{*}{$\begin{array}{c}\text { Direction } \\
\text { of Transverse Strain } \\
\text { (Poisson's Ratio } \\
\text { Tensor Designation) }\end{array}$} & \multirow[b]{2}{*}{ Loading } & \multicolumn{9}{|c|}{ Poisson's Ratio (Standard Deviation) } \\
\hline & & & & & & & $200 \mathrm{psi}$ & $250 \mathrm{psi}$ & $400 \mathrm{psi}$ & $500 \mathrm{psi}$ & $600 \mathrm{psi}$ & $750 \mathrm{psi}$ & $800 \mathrm{psi}$ & $1000 \mathrm{psi}$ & $1200 \mathrm{psi}$ \\
\hline $\begin{array}{l}6399-8-B / \\
5651-63 / \\
(22 / 266)\end{array}$ & $\begin{array}{l}0.5 \times 0.5 \\
\times 6 \text { long }\end{array}$ & $4\left(90^{\circ}\right.$ apart $)$ & MLC & Axial & $\stackrel{\text { Radial }}{\left(v_{31}=v_{32}\right)}$ & \begin{tabular}{|c}
1 \\
2 \\
3 \\
4 \\
5 \\
6 \\
7 \\
8 \\
8 \\
Mean
\end{tabular} & $\begin{array}{l}0.120(0.009) \\
0.118(0.008) \\
0.115(0.010) \\
0.125(0.007) \\
0.121(0.005) \\
0.131(0.003) \\
0.123(0.011) \\
0.123(0.009) \\
0.123(0.009)\end{array}$ & & $\begin{array}{l}0.123(0.008) \\
0.124(0.010) \\
0.122(0.009) \\
0.125(0.008) \\
0.124(0.008) \\
0.127(0.006) \\
0.125(0.007) \\
0.125(0.009) \\
0.125(0.007)\end{array}$ & & $\begin{array}{l}0.123(0.008) \\
0.125(0.009) \\
0.124(0.009) \\
0.125(0.008) \\
0.125(0.009) \\
0.128(0.007) \\
0.126(0.009) \\
0.126(0.008) \\
0.126(0.008)\end{array}$ & 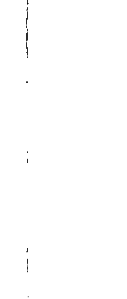 & 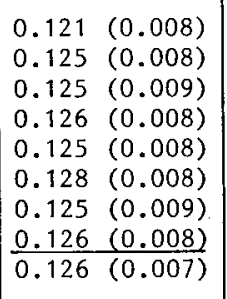 & & \\
\hline
\end{tabular}


TABLE B-19

POISSON'S RATIO: TS- 1240

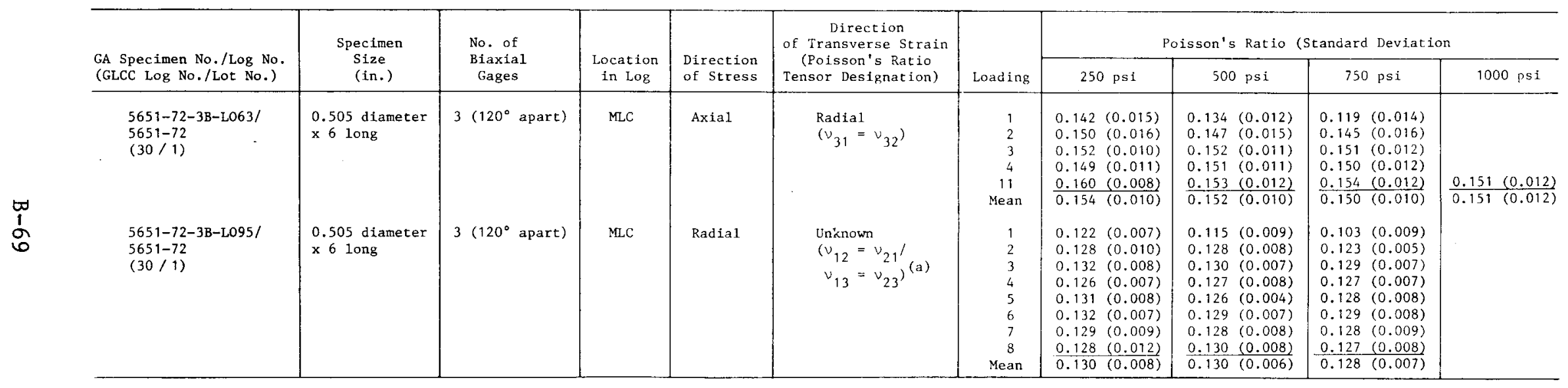

(a) Orfentation of sample in log with respect to directions of measured trantsverse strains was unknowi. poisson's ratio values shown represent mean of various components $\left(v_{12}, v_{21}, v_{13}, v_{23}\right)$ indicated. 
TABLE B-20

THERMAL EXPANSIVITY OF H-451, LOT 266

[Log 6484-28 (GLCC 18), whole $\log$ density $=1.77 \mathrm{~g} / \mathrm{cm}^{3}{ }^{(a)}$ ]

\begin{tabular}{|c|c|c|}
\hline $\begin{array}{c}\text { GA } \\
\text { Specimen } \\
\text { No. } \\
\end{array}$ & $\begin{array}{l}\text { Location in } \\
\text { Log and } \\
\text { Orientation }\end{array}$ & $\begin{array}{c}\text { Mean Coefficient of Thermal } \\
\text { Expansion, } \alpha \times 10^{6}{ }^{\circ} \mathrm{C}^{-1} \\
\left(22^{\circ}-500^{\circ} \mathrm{C}\right) \\
\end{array}$ \\
\hline $\begin{array}{r}353 \\
354 \\
355 \\
356 \\
357\end{array}$ & MLC, axia1 & $\begin{array}{l}3.44 \\
3.44 \\
3.49 \\
3.42 \\
3.46 \\
\end{array}$ \\
\hline $\begin{array}{l}\text { Mean } \\
\text { Std. Dev. }\end{array}$ & $\downarrow$ & $\begin{array}{l}3.45 \\
0.03\end{array}$ \\
\hline $\begin{array}{r}341 \\
342 \\
343 \\
344 \\
345 \\
346\end{array}$ & MLC, radial & $\begin{array}{l}4.43 \\
4.36 \\
4.40 \\
4.20 \\
4.60 \\
4.45 \\
\end{array}$ \\
\hline $\begin{array}{l}\text { Mean } \\
\text { Std. Dev. }\end{array}$ & 1 & $\begin{array}{l}4.41 \\
0.13\end{array}$ \\
\hline $\begin{array}{r}359 \\
360 \\
361 \\
362 \\
363 \\
364\end{array}$ & MLE, axial & $\begin{array}{l}3.36 \\
3.52 \\
3.51 \\
3.43 \\
3.48 \\
3.41 \\
\end{array}$ \\
\hline $\begin{array}{l}\text { Mean } \\
\text { Std. Dev. }\end{array}$ & $f$ & $\begin{array}{l}3.45 \\
0.06\end{array}$ \\
\hline $\begin{array}{r}347 \\
348 \\
349 \\
350 \\
351 \\
352\end{array}$ & MLE, radial & $\begin{array}{l}4.34 \\
4.58 \\
4.61 \\
4.63 \\
4.53 \\
4.25 \\
\end{array}$ \\
\hline $\begin{array}{l}\text { Mean } \\
\text { Std. Dev. }\end{array}$ & $\downarrow$ & $\begin{array}{l}4.49 \\
0.16\end{array}$ \\
\hline
\end{tabular}

(a) Measured at GLCC. 
TABLE $\quad B-21$

THERMAL EXPANSIVITY OF H-451, LOT 408

[ $\log 5651-86$ (GLCC 52), whole $\log$ density $=1.74 \mathrm{~g} / \mathrm{cm}^{3(a)}$ ]

\begin{tabular}{|c|c|c|}
\hline $\begin{array}{l}\text { GA } \\
\text { Specimen } \\
\text { No. }\end{array}$ & $\begin{array}{l}\text { Location in } \\
\text { Log and } \\
\text { Orientation }\end{array}$ & $\begin{array}{l}\text { Mean Coefficient of Thermal } \\
\text { Expansion, (b) } \alpha \times 16^{\circ} \mathrm{C}^{-1} \\
\left(22^{\circ}-500^{\circ} \mathrm{C}\right)\end{array}$ \\
\hline $\begin{array}{r}5651-86-3 \mathrm{~A}-001 \mathrm{~B} \\
-002 \mathrm{~B} \\
-010 \mathrm{~B} \\
-011 \mathrm{~B} \\
-3 \mathrm{~B}-001 \mathrm{~B} \\
-002 \mathrm{~B} \\
-010 \mathrm{~B} \\
-011 \mathrm{~B}\end{array}$ & MLC, axial & $\begin{array}{l}4.00(4.36) \\
4.35(4.26) \\
4.41 \\
4.14 \\
4.08(4.44) \\
4.27(4.35) \\
4.04 \\
4.16\end{array}$ \\
\hline $\begin{array}{l}\text { Mean } \\
\text { Std. Dev. }\end{array}$ & 1 & $\begin{array}{l}4.18 \\
0.15\end{array}$ \\
\hline $\begin{array}{r}5651-86-3 \mathrm{~A}-031 \mathrm{~B} \\
-032 \mathrm{~B} \\
-040 \mathrm{~B} \\
-041 \mathrm{~B} \\
-3 \mathrm{~B}-031 \mathrm{~B} \\
-032 \mathrm{~B} \\
-040 \mathrm{~B} \\
-041 \mathrm{~B} \\
-031 \mathrm{~A} \\
-041 \mathrm{~A}\end{array}$ & MLC, radial & $\begin{array}{ll}4.53 & (4.73) \\
4.81 & (4.77) \\
4.62 & \\
4.82 & \\
4.37 & (4.66) \\
4.47 & (4.44) \\
4.72 & \\
4.64 & \\
4.83 & \\
4.46 & \\
\end{array}$ \\
\hline $\begin{array}{l}\text { Mean } \\
\text { Std. Dev. }\end{array}$ & 1 & $\begin{array}{l}4.63 \\
0.17\end{array}$ \\
\hline $\begin{array}{r}5651-86-3 \mathrm{~A}-101 \mathrm{~B} \\
-102 \mathrm{~B} \\
-110 \mathrm{~B} \\
-111 \mathrm{~B} \\
-3 \mathrm{~B}-101 \mathrm{~A} \\
-101 \mathrm{~B} \\
-111 \mathrm{~A} \\
-111 \mathrm{~B} \\
-102 \mathrm{~B} \\
-110 \mathrm{~B}\end{array}$ & MLE, axial & $\begin{array}{l}4.20(4.39) \\
4.36 \\
4.21 \\
4.11 \quad(4.19) \\
4.10 \\
4.11 \quad(4.31) \\
4.10 \\
4.05 \quad(4.31) \\
4.34 \\
4.28\end{array}$ \\
\hline $\begin{array}{l}\text { Mean } \\
\text { Std. Dev. }\end{array}$ & 1 & $\begin{array}{l}4.19 \\
0.11\end{array}$ \\
\hline $\begin{array}{r}5651-86-3 \mathrm{~A}-131 \mathrm{~A} \\
-131 \mathrm{~B} \\
-141 \mathrm{~A} \\
-141 \mathrm{~B} \\
-132 \mathrm{~B} \\
-140 \mathrm{~B} \\
3 \mathrm{~B}-131 \mathrm{~A} \\
-131 \mathrm{~B} \\
-141 \mathrm{~A} \\
-141 \mathrm{~B} \\
-132 \mathrm{~B} \\
-140 \mathrm{~B}\end{array}$ & MLE, radial & $\begin{array}{l}4.62 \\
4.16(4.40) \\
4.80 \\
4.35(4.64) \\
4.66 \\
4.62 \\
4.84 \\
4.65(4.77) \\
4.69 \\
4.27(4.40) \\
4.72 \\
4.43 \\
\end{array}$ \\
\hline $\begin{array}{l}\text { Mean } \\
\text { Std. Dev. }\end{array}$ & 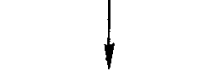 & $\begin{array}{l}4.57 \\
0.21\end{array}$ \\
\hline
\end{tabular}

(a) Measured at GLCC.

(b) Values in parentheses are repeat measurements. 
TABLE B-22

THERMAL EXPANSIVITY OF H-451, LOT 426

[Log 6484-33 (GLCC 92), whole $\log$ density $\left.=1.75 \mathrm{~g} / \mathrm{cm}^{3}{ }^{(a)}\right]$

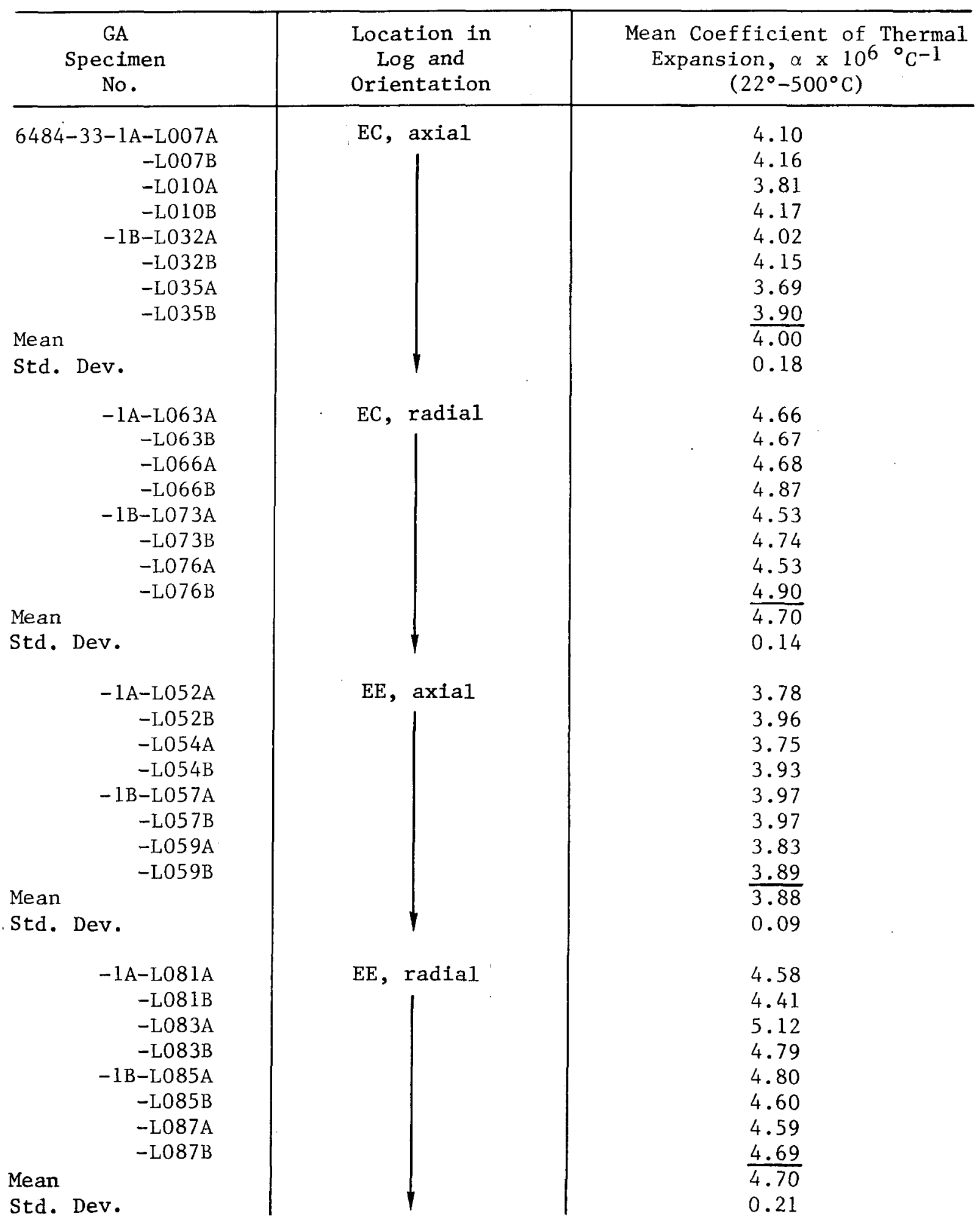


TABLE B-22 (Continued)

\begin{tabular}{|c|c|c|}
\hline $\begin{array}{l}\text { GA } \\
\text { Specimen } \\
\text { No. }\end{array}$ & $\begin{array}{l}\text { Location in } \\
\quad \text { Log and } \\
\text { Orientation }\end{array}$ & $\begin{array}{l}\text { Mean Coefficient of Thermal } \\
\text { Expansion, } \alpha \times 10^{6}{ }^{\circ} \mathrm{C}^{-1} \\
\left(22^{\circ}-500^{\circ} \mathrm{C}\right)\end{array}$ \\
\hline $\begin{array}{l}\begin{array}{r}6484-33-3 A-L 107 A \\
-L 107 B \\
-L 110 A \\
-L 110 B \\
-3 B-L 132 A \\
-L 132 B \\
-L 135 A \\
-L 135 B\end{array} \\
\text { Mean } \\
\text { Std. Dev. }\end{array}$ & $\begin{array}{c}\text { MLC, axial } \\
1\end{array}$ & $\begin{array}{l}4.19 \\
4.27 \\
4.26 \\
4.09 \\
3.96 \\
3.90 \\
4.04 \\
\frac{3.94}{4.08} \\
0.14\end{array}$ \\
\hline $\begin{array}{r}-3 \mathrm{~A}-\mathrm{L} 163 \mathrm{~A} \\
-\mathrm{L} 163 \mathrm{~B} \\
-\mathrm{L} 166 \mathrm{~A} \\
-\mathrm{L} 166 \mathrm{~B} \\
-3 \mathrm{~B}-\mathrm{L} 173 \mathrm{~A} \\
-\mathrm{L} 173 \mathrm{~B} \\
-\mathrm{L} 176 \mathrm{~A} \\
-\mathrm{L} 176 \mathrm{~B}\end{array}$ & MLC, radia1 & $\begin{array}{l}4.62 \\
4.35 \\
5.19 \\
4.37 \\
4.37 \\
4.29 \\
4.45 \\
4.45 \\
\end{array}$ \\
\hline $\begin{array}{l}\text { Mean } \\
\text { Std. Dev. }\end{array}$ & 1 & $\begin{array}{l}\overline{4.51} \\
0.29\end{array}$ \\
\hline $\begin{array}{r}-3 \mathrm{~A}-\mathrm{L} 152 \mathrm{~A} \\
-\mathrm{L} 152 \mathrm{~B} \\
-\mathrm{L} 154 \mathrm{~A} \\
-\mathrm{L} 154 \mathrm{~B} \\
-3 \mathrm{~B}-\mathrm{L} 157 \mathrm{~A} \\
-\mathrm{L} 157 \mathrm{~B} \\
-\mathrm{L} 159 \mathrm{~A} \\
-\mathrm{L} 159 \mathrm{~B}\end{array}$ & MLE, axia1 & $\begin{array}{l}3.78 \\
3.92 \\
3.81 \\
4.24 \\
3.70 \\
3.58 \\
3.80 \\
4.03\end{array}$ \\
\hline $\begin{array}{l}\text { Mean } \\
\text { Std. Dev. }\end{array}$ & 1 & $\begin{array}{l}\overline{3.86} \\
0.20\end{array}$ \\
\hline $\begin{array}{r}-3 \mathrm{~A}-\mathrm{L} 181 \mathrm{~A} \\
-\mathrm{L} 181 \mathrm{~B} \\
-\mathrm{L} 183 \mathrm{~A} \\
-\mathrm{L} 183 \mathrm{~B} \\
-3 \mathrm{~B}-\mathrm{L} 185 \mathrm{~A} \\
-\mathrm{L} 185 \mathrm{~B} \\
-\mathrm{L} 187 \mathrm{~A} \\
-\mathrm{L} 187 \mathrm{~B}\end{array}$ & MLE, radial & $\begin{array}{l}4.80 \\
4.61 \\
4.59 \\
4.85 \\
4.66 \\
4.60 \\
4.65 \\
4.43\end{array}$ \\
\hline $\begin{array}{l}\text { Mean } \\
\text { Std. Dev. }\end{array}$ & $\downarrow$ & $\begin{array}{l}4.65 \\
0.13\end{array}$ \\
\hline
\end{tabular}

(a) Measured at GLCC. 
TABLE B-23

THERMAL EXPANSIVITY OF H-451, LOT 426
$\left[\log 6484-34\right.$ (GLCC 198), whole $\log$ density $\left.=1.71 \mathrm{~g} / \mathrm{cm}^{3}{ }^{(a)}\right]$

\begin{tabular}{|c|c|c|}
\hline $\begin{array}{l}\text { GA } \\
\text { Specimen } \\
\text { No. }\end{array}$ & $\begin{array}{l}\text { Location in } \\
\text { Log and } \\
\text { Orientation }\end{array}$ & $\begin{array}{c}\text { Mean Coefficient of Thermal } \\
\text { Expansion, } \alpha \times 16^{\circ} \mathrm{C}^{-1} \\
\left(22^{\circ}-500^{\circ} \mathrm{C}\right)\end{array}$ \\
\hline $\begin{array}{rr}6484-34-1 A-L 007 A \\
-L 007 B \\
-L 010 A \\
-L 010 B \\
-1 B-L 032 A \\
-L 032 B \\
-L 035 A \\
& -L 035 B \\
& \\
\text { Mean } & \\
\text { Std. Dev. } & \end{array}$ & EC, axial & $\begin{array}{l}4.24 \\
4.32 \\
4.46 \\
4.22 \\
4.31 \\
4.40 \\
4.24 \\
4.29 \\
4.31 \\
0.08\end{array}$ \\
\hline 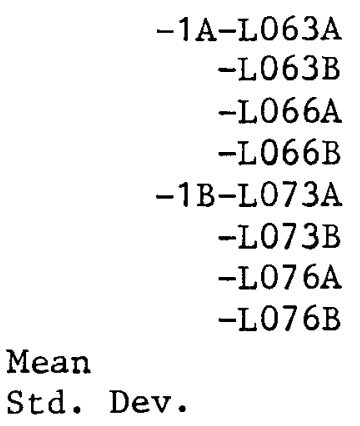 & EC, radial & $\begin{array}{l}5.02 \\
4.91 \\
4.50 \\
4.33 \\
4.41 \\
4.36 \\
4.35 \\
4.52 \\
4.55 \\
0.27\end{array}$ \\
\hline 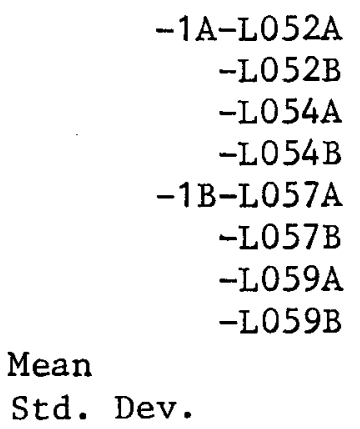 & $\downarrow$ & $\begin{array}{l}3.88 \\
4.13 \\
4.07 \\
4.17 \\
4.24 \\
4.19 \\
4.09 \\
4.25 \\
4.13 \\
0.12\end{array}$ \\
\hline 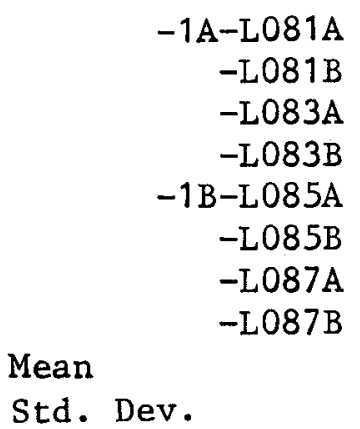 & $\mathrm{EE}$, radial & $\begin{array}{l}4.62 \\
4.52 \\
4.66 \\
4.75 \\
4.70 \\
4.53 \\
4.52 \\
4.45 \\
4.59 \\
0.10\end{array}$ \\
\hline
\end{tabular}


TABLE B-23 (Continued)

\begin{tabular}{|c|c|c|}
\hline $\begin{array}{l}\text { GA } \\
\text { Specimen } \\
\text { No. }\end{array}$ & $\begin{array}{l}\text { Location in } \\
\text { Log and } \\
\text { Orientation }\end{array}$ & $\begin{array}{c}\text { Mean Coefficient of Thermal } \\
\text { Expansion, } \alpha \times 106^{\circ} \mathrm{C}^{-1} \\
\left(22^{\circ}-500^{\circ} \mathrm{C}\right)\end{array}$ \\
\hline $\begin{array}{r}\text { 6484-34-3A-L107A } \\
\text {-L107B } \\
\text {-L110A } \\
\text {-L110B } \\
-3 B-L 132 A \\
-L 132 B \\
\text {-L135A } \\
\text {-L135B } \\
\\
\text { Mean } \\
\text { Std. Dev. }\end{array}$ & MLC, axial & $\begin{array}{l}4.06 \\
4.13 \\
3.98 \\
4.31 \\
4.03 \\
4.07 \\
4.13 \\
4.13 \\
4.10 \\
0.10\end{array}$ \\
\hline 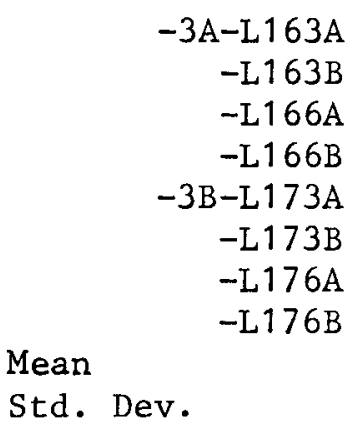 & MLC, radial & $\begin{array}{l}4.75 \\
4.47 \\
4.73 \\
4.72 \\
4.88 \\
4.24 \\
4.68 \\
4.56 \\
4.63 \\
0.20\end{array}$ \\
\hline $\begin{array}{r}-3 A-L 152 A \\
-L 152 B \\
-L 154 A \\
-L 154 B \\
-3 B-L 157 A \\
-L 157 B \\
-L 159 A \\
-L 159 B\end{array}$ & MLE, axial & $\begin{array}{l}3.69 \\
4.08 \\
3.75 \\
3.88 \\
4.21 \\
4.02 \\
3.70 \\
4.01\end{array}$ \\
\hline $\begin{array}{l}\text { Mean } \\
\text { Std. Dev. }\end{array}$ & 1 & $\begin{array}{l}3.92 \\
0.19\end{array}$ \\
\hline 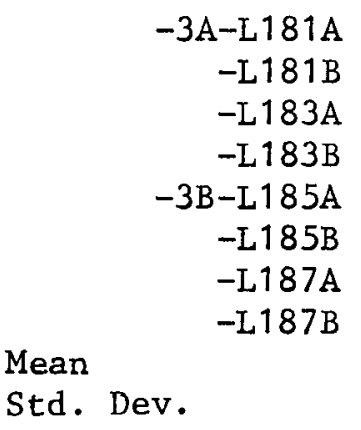 & MLE, radial & $\begin{array}{l}4.61 \\
4.63 \\
5.14 \\
4.66 \\
4.79 \\
4.77 \\
4.61 \\
4.42 \\
4.66 \\
0.25\end{array}$ \\
\hline
\end{tabular}

(a) Measured at GLCC. 
TABLE B-24

THERMAL EXPANSIVITY OF H-451, LOT 426

[ $\log 6484-41$ (GLCC 184), whole $\log$ density $=1.72 \mathrm{~g} / \mathrm{cm}^{3}{ }^{(a)}$ ]

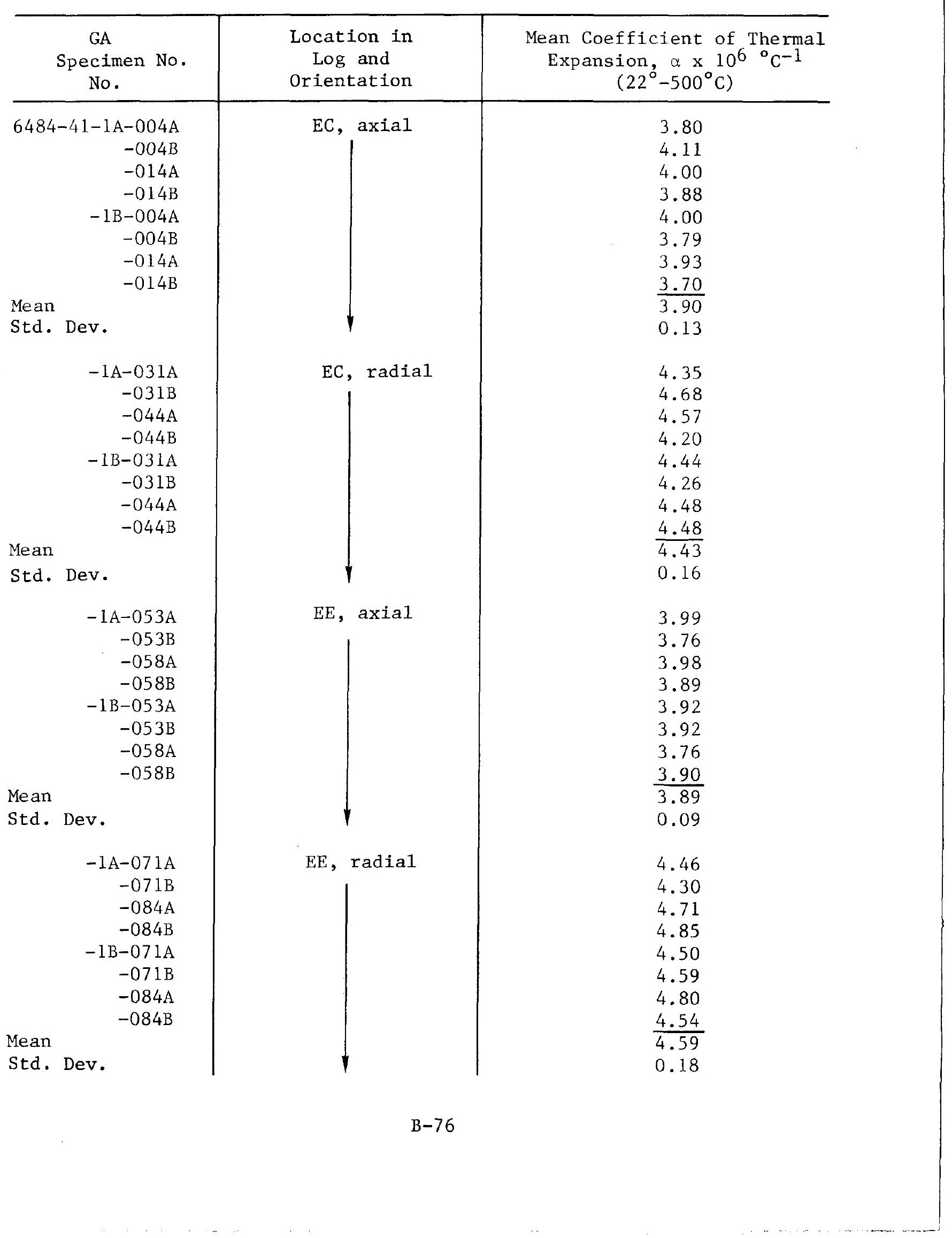


TABLE B-24 (Continued)

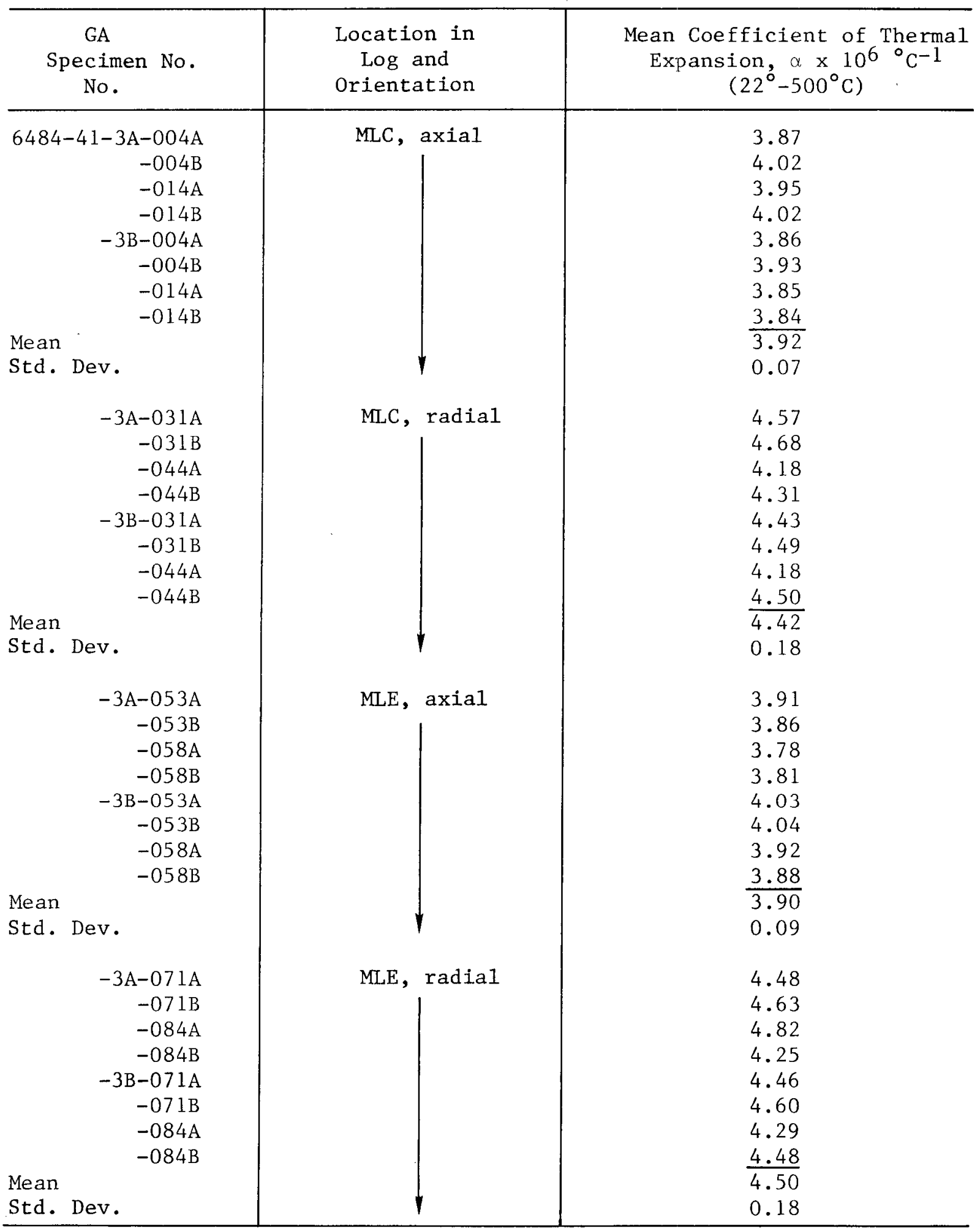

(a) Measured at GLCC. 
TABLE $B-25$

THERMAL EXPANSIVITY OF TS-1240, LOT 1

[Log 5651-72 (UCC 32), whole log density $=1.77 \mathrm{~g} / \mathrm{cm}^{3(\mathrm{a})}$ ]

\begin{tabular}{|c|c|c|}
\hline $\begin{array}{l}\text { GA } \\
\text { Specimen } \\
\text { No. }\end{array}$ & $\begin{array}{l}\text { Location in Log } \\
\text { and Orientation }\end{array}$ & $\begin{array}{c}\text { Mean Coefficient of Thermal } \\
\text { Expansion, } \alpha \times 10^{6}{ }^{\circ} \mathrm{C}^{-1} \\
\left(22^{\circ}-500^{\circ} \mathrm{C}\right)\end{array}$ \\
\hline $\begin{array}{r}5651-72-1 \mathrm{~A}-007 \mathrm{~A} \\
-007 \mathrm{~B} \\
-010 \mathrm{~A} \\
-010 \mathrm{~B} \\
-1 \mathrm{~B}-032 \mathrm{~A} \\
-032 \mathrm{~B} \\
-035 \mathrm{~A} \\
-035 \mathrm{~B}\end{array}$ & EC, axial & $\begin{array}{l}4.51 \\
4.43 \\
4.60 \\
4.45 \\
4.36 \\
4.40 \\
4.64 \\
4.52 \\
\end{array}$ \\
\hline $\begin{array}{l}\text { Mean } \\
\text { Std. Dev. }\end{array}$ & & $\begin{array}{l}4.48 \\
0.097\end{array}$ \\
\hline $\begin{array}{r}5651-72-1 \mathrm{~A}-063 \mathrm{~A} \\
-063 \mathrm{~B} \\
-066 \mathrm{~A} \\
-066 \mathrm{~B} \\
-1 \mathrm{~B}-073 \mathrm{~A} \\
-073 \mathrm{~B} \\
-076 \mathrm{~A} \\
-076 \mathrm{~B}\end{array}$ & EC, radial & $\begin{array}{l}4.70 \\
5.08 \\
4.75 \\
4.61 \\
4.80 \\
4.83 \\
4.66 \\
5.33 \\
\end{array}$ \\
\hline $\begin{array}{l}\text { Mean } \\
\text { Std. Dev. }\end{array}$ & 1 & $\begin{array}{l}4.84 \\
0.243\end{array}$ \\
\hline $\begin{array}{r}5651-72-1 \mathrm{~A}-052 \mathrm{~A} \\
-052 \mathrm{~B} \\
-054 \mathrm{~A} \\
-054 \mathrm{~B} \\
-1 \mathrm{~B}-057 \mathrm{~A} \\
-057 \mathrm{~B} \\
-059 \mathrm{~A} \\
-059 \mathrm{~B}\end{array}$ & $\mathrm{EE}$, axial & $\begin{array}{l}3.96 \\
4.45 \\
4.32 \\
4.75 \\
4.28 \\
4.48 \\
4.96 \\
4.73 \\
\end{array}$ \\
\hline $\begin{array}{l}\text { Mean } \\
\text { Std. Dev. }\end{array}$ & 1 & $\begin{array}{l}4.49 \\
0.317\end{array}$ \\
\hline $\begin{array}{r}5651-72-1 \mathrm{~A}-081 \mathrm{~A} \\
-081 \mathrm{~B} \\
-083 \mathrm{~A} \\
-083 \mathrm{~B} \\
-1 \mathrm{~B}-085 \mathrm{~A} \\
-085 \mathrm{~B} \\
-087 \mathrm{~A} \\
-087 \mathrm{~B}\end{array}$ & EE, radial & $\begin{array}{l}4.77 \\
5.57 \\
5.07 \\
5.04 \\
5.00 \\
5.16 \\
4.31 \\
4.83\end{array}$ \\
\hline $\begin{array}{l}\text { Mean } \\
\text { Std. Dev. }\end{array}$ & & $\begin{array}{l}4.96 \\
0.360\end{array}$ \\
\hline
\end{tabular}


TABLE B-25 (Continued)

\begin{tabular}{|c|c|c|}
\hline $\begin{array}{l}\text { GA } \\
\text { Specimen } \\
\text { No. }\end{array}$ & $\begin{array}{l}\text { Location in Log } \\
\text { and Orientation }\end{array}$ & $\begin{array}{c}\text { Mean Coefficient of Thermal } \\
\text { Expansion, } \alpha \times 10^{\circ} \mathrm{C}^{-1} \\
\left(22^{\circ}-500^{\circ} \mathrm{C}\right)\end{array}$ \\
\hline $\begin{array}{r}5651-72-3 \mathrm{~A}-107 \mathrm{~A} \\
-107 \mathrm{~B} \\
-110 \mathrm{~A} \\
-110 \mathrm{~B} \\
-3 \mathrm{~B}-132 \mathrm{~A} \\
-132 \mathrm{~B} \\
-135 \mathrm{~A} \\
-135 \mathrm{~B}\end{array}$ & QLC, axial & $\begin{array}{l}4.16 \\
4.29 \\
4.00 \\
4.35 \\
3.83 \\
4.10 \\
3.78 \\
3.90\end{array}$ \\
\hline $\begin{array}{l}\text { Mean } \\
\text { Std. Dev. }\end{array}$ & $\eta$ & $\begin{array}{l}4.05 \\
0.209\end{array}$ \\
\hline $\begin{array}{r}5651-72-3 \mathrm{~A}-163 \mathrm{~A} \\
-163 \mathrm{~B} \\
-166 \mathrm{~A} \\
-166 \mathrm{~B} \\
-3 \mathrm{~B}-173 \mathrm{~A} \\
-173 \mathrm{~B} \\
-176 \mathrm{~A} \\
-176 \mathrm{~B}\end{array}$ & QLC, radial & $\begin{array}{l}4.64 \\
4.45 \\
4.42 \\
4.63 \\
4.50 \\
4.31 \\
4.55 \\
4.52\end{array}$ \\
\hline $\begin{array}{l}\text { Mean } \\
\text { Std. Dev. }\end{array}$ & $\gamma$ & $\begin{array}{l}4.50 \\
0.109\end{array}$ \\
\hline $\begin{array}{r}5651-72-3 \mathrm{~A}-152 \mathrm{~A} \\
-152 \mathrm{~B} \\
-154 \mathrm{~A} \\
-154 \mathrm{~B} \\
-3 \mathrm{~B}-157 \mathrm{~A} \\
-157 \mathrm{~B} \\
-159 \mathrm{~A} \\
-159 \mathrm{~B}\end{array}$ & QLE, axial & $\begin{array}{l}3.99 \\
4.02 \\
4.45 \\
3.92 \\
4.16 \\
4.19 \\
4.17 \\
4.03 \\
\end{array}$ \\
\hline $\begin{array}{l}\text { Mean } \\
\text { Std. Dev. }\end{array}$ & 1 & $\begin{array}{l}4.12 \\
0.165\end{array}$ \\
\hline $\begin{array}{r}5651-72-3 \mathrm{~A}-181 \mathrm{~A} \\
-181 \mathrm{~B} \\
-183 \mathrm{~A} \\
-183 \mathrm{~B} \\
-3 \mathrm{~B}-185 \mathrm{~A} \\
-185 \mathrm{~B} \\
-187 \mathrm{~A} \\
-187 \mathrm{~B}\end{array}$ & QLE, radial & $\begin{array}{l}4.90 \\
4.44 \\
5.07 \\
4.65 \\
4.65 \\
4.50 \\
4.65 \\
4.63 \\
\end{array}$ \\
\hline $\begin{array}{l}\text { Mean } \\
\text { Std. Dev. }\end{array}$ & $\gamma$ & $\begin{array}{l}4.68 \\
0.205\end{array}$ \\
\hline
\end{tabular}

(a) Measured at UCC. 
TABLE B-26

THERMAL EXPANSIVITY OF TS-1240, LOT 1
[Log 5651-73 (UCC 40), whole log density $=1.80 \mathrm{~g} / \mathrm{cm}^{3(a)}$ ]

\begin{tabular}{|c|c|c|}
\hline $\begin{array}{l}\text { GA } \\
\text { Specimen } \\
\text { No. }\end{array}$ & $\begin{array}{l}\text { Location in Log } \\
\text { and Orientation }\end{array}$ & $\begin{array}{l}\text { Mean Coefficient of Thermal } \\
\text { Expansion, (b) } \alpha \times 10^{6}{ }^{\circ} \mathrm{C}^{-1} \\
\left(22^{\circ}-300^{\circ} \mathrm{C}\right)\end{array}$ \\
\hline $\begin{array}{r}5651-73-3 \mathrm{~A}-001 \mathrm{~B} \\
-002 \mathrm{~B} \\
-010 \mathrm{~B} \\
-011 \mathrm{~B} \\
-3 \mathrm{~B}-001 \mathrm{~B} \\
-002 \mathrm{~B} \\
-010 \mathrm{~B} \\
-011 \mathrm{~B}\end{array}$ & MLC, axial & $\begin{array}{l}4.17(4.04) \\
4.22 \\
4.20 \\
4.46(4.18) \\
3.97(4.22) \\
4.09 \\
4.67 \\
4.29(3.99)\end{array}$ \\
\hline $\begin{array}{l}\text { Mean } \\
\text { Std. Dev. }\end{array}$ & 1 & $\begin{array}{l}4.26 \\
0.22\end{array}$ \\
\hline $\begin{array}{r}5651-73-3 \mathrm{~A}-031 \mathrm{~B} \\
-032 \mathrm{~B} \\
-040 \mathrm{~B} \\
-041 \mathrm{~B} \\
3 \mathrm{~B}-031 \mathrm{~B} \\
-032 \mathrm{~B} \\
-0408 \\
-041 \mathrm{~B}\end{array}$ & MLC, radial & 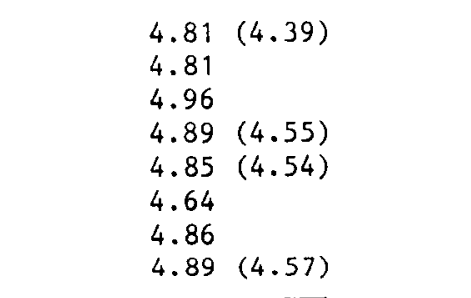 \\
\hline $\begin{array}{l}\text { Mean } \\
\text { Std. Dev. }\end{array}$ & 1 & $\begin{array}{l}4.84 \\
0.09\end{array}$ \\
\hline $\begin{array}{r}5651-73-3 \mathrm{~A}-101 \mathrm{~B} \\
-102 \mathrm{~B} \\
-111 \mathrm{~A} \\
-111 \mathrm{~B} \\
-110 \mathrm{~B} \\
3 \mathrm{~B}-101 \mathrm{~A} \\
-101 \mathrm{~B} \\
-111 \mathrm{~A} \\
-111 \mathrm{~B} \\
-102 \mathrm{~B} \\
-110 \mathrm{~B}\end{array}$ & MLE, axial & $\begin{array}{l}4.29(4.12) \\
4.61 \\
3.86 \\
4.04(4.08) \\
4.51 \\
4.05 \\
4.15(4.02) \\
4.27 \\
4.25 \\
4.63 \\
4.45\end{array}$ \\
\hline $\begin{array}{l}\text { Mean } \\
\text { Std. Dev. }\end{array}$ & 1 & $\begin{array}{l}4.28 \\
0.25\end{array}$ \\
\hline $\begin{array}{r}5651-3 \mathrm{~A}-131 \mathrm{~A} \\
-131 \mathrm{~B} \\
-141 \mathrm{~A} \\
-141 \mathrm{~B} \\
-132 \mathrm{~B} \\
-140 \mathrm{~B} \\
3 \mathrm{~B}-131 \mathrm{~A} \\
-131 \mathrm{~B} \\
-141 \mathrm{~A} \\
-141 \mathrm{~B} \\
-132 \mathrm{~B} \\
-140 \mathrm{~B}\end{array}$ & MLE, radial & $\begin{array}{l}4.28 \\
4.78(4.57) \\
4.56 \\
4.86(4.45) \\
4.70 \\
5.15 \\
4.53 \\
4.83(4.54) \\
4.81 \\
4.70(4.41) \\
4.80 \\
4.78 \\
\end{array}$ \\
\hline $\begin{array}{l}\text { Mean } \\
\text { Std. Dev. }\end{array}$ & 1 & $\begin{array}{l}4.73 \\
0.21\end{array}$ \\
\hline
\end{tabular}

(a) Measured at UCC.

(b) Values in parentheses are repeat measurements. 
TABLE $\quad B-27$

THERMAL EXPANSIVITY OF TS-1240, LOT 1

[ $\log 5651-74$ (UCC 42), whole $\log$ density $\left.=1.82 \mathrm{~g} / \mathrm{cm}^{3}{ }^{(\mathrm{a})}\right]$

\begin{tabular}{|c|c|c|}
\hline $\begin{array}{l}\text { GA } \\
\text { Specimen } \\
\text { No. }\end{array}$ & $\begin{array}{l}\text { Location in Log } \\
\text { and Orientation }\end{array}$ & $\begin{array}{c}\text { Mean Coefficient of Thermal } \\
\text { Expansion, } \alpha \times 10^{6}{ }^{\circ} \mathrm{C}^{-1} \\
\left(22^{\circ}-500^{\circ} \mathrm{C}\right)\end{array}$ \\
\hline $\begin{array}{r}5651-74-1 \mathrm{~A}-007 \mathrm{~A} \\
-007 \mathrm{~B} \\
-010 \mathrm{~A} \\
-010 \mathrm{~B} \\
-1 \mathrm{~B}-032 \mathrm{~A} \\
-032 \mathrm{~B} \\
-035 \mathrm{~A} \\
-035 \mathrm{~B}\end{array}$ & EC, axial & $\begin{array}{l}4.25 \\
4.09 \\
4.28 \\
4.49 \\
4.25 \\
4.55 \\
4.25 \\
4.15\end{array}$ \\
\hline $\begin{array}{l}\text { Mean } \\
\text { Std. Dev. }\end{array}$ & 1 & $\begin{array}{l}4.29 \\
0.16\end{array}$ \\
\hline $\begin{array}{r}5651-74-1 \mathrm{~A}-063 \mathrm{~A} \\
-063 \mathrm{~B} \\
-066 \mathrm{~A} \\
-066 \mathrm{~B} \\
-1 \mathrm{~B}-073 \mathrm{~A} \\
-073 \mathrm{~B} \\
-076 \mathrm{~A} \\
-076 \mathrm{~B}\end{array}$ & EC, radial & $\begin{array}{l}4.45 \\
4.52 \\
4.75 \\
4.75 \\
4.56 \\
4.67 \\
4.71 \\
4.71\end{array}$ \\
\hline $\begin{array}{l}\text { Mean } \\
\text { Std. Dev. }\end{array}$ & 1 & $\begin{array}{l}4.63 \\
0.11\end{array}$ \\
\hline $\begin{array}{r}5651-74-1 \mathrm{~A}-052 \mathrm{~A} \\
-052 \mathrm{~B} \\
-054 \mathrm{~A} \\
-054 \mathrm{~B} \\
-1 \mathrm{~B}-057 \mathrm{~A} \\
-057 \mathrm{~B} \\
-059 \mathrm{~A} \\
-059 \mathrm{~B}\end{array}$ & $\mathrm{EE}$, axial & $\begin{array}{l}4.45 \\
4.68 \\
4.23 \\
4.16 \\
4.20 \\
4.17 \\
4.20 \\
4.16\end{array}$ \\
\hline $\begin{array}{l}\text { Mean } \\
\text { Std. Dev. }\end{array}$ & 1 & $\begin{array}{l}4.28 \\
0.19\end{array}$ \\
\hline $\begin{array}{r}5651-74-1 \mathrm{~A}-081 \mathrm{~A} \\
-081 \mathrm{~B} \\
-083 \mathrm{~A} \\
-083 \mathrm{~B} \\
-1 \mathrm{~B}-085 \mathrm{~A} \\
-085 \mathrm{~B} \\
-087 \mathrm{~A} \\
-087 \mathrm{~B}\end{array}$ & $\mathrm{EE}$, radial & $\begin{array}{l}4.75 \\
4.86 \\
4.94 \\
4.65 \\
4.83 \\
4.85 \\
5.01 \\
4.83\end{array}$ \\
\hline $\begin{array}{l}\text { Mean } \\
\text { Std. Dev. }\end{array}$ & 1 & $\begin{array}{l}4.84 \\
0.11\end{array}$ \\
\hline
\end{tabular}


TABLE B-27 (Continued)

\begin{tabular}{|c|c|c|}
\hline $\begin{array}{l}\text { GA } \\
\text { Specimen } \\
\text { No. }\end{array}$ & $\begin{array}{l}\text { Location in Log } \\
\text { and Orientation }\end{array}$ & $\begin{array}{c}\text { Mean Coefficient of Thermal } \\
\text { Expansion, } \alpha \times 10^{\circ}{ }^{\circ} \mathrm{C}^{-1} \\
\left(22^{\circ}-500^{\circ} \mathrm{C}\right)\end{array}$ \\
\hline $\begin{array}{r}5651-74-3 \mathrm{~A}-107 \mathrm{~A} \\
-107 \mathrm{~B} \\
-110 \mathrm{~A} \\
-110 \mathrm{~B} \\
3 \mathrm{~B}-132 \mathrm{~A} \\
-132 \mathrm{~B} \\
-135 \mathrm{~A} \\
-135 \mathrm{~B}\end{array}$ & MLC, axial & $\begin{array}{l}4.38 \\
4.22 \\
4.39 \\
4.27 \\
4.33 \\
4.33 \\
4.31 \\
4.41\end{array}$ \\
\hline $\begin{array}{l}\text { Mean } \\
\text { Std. Dev. }\end{array}$ & 1 & $\begin{array}{l}4.33 \\
0.06\end{array}$ \\
\hline $\begin{array}{r}5651-74-3 \mathrm{~A}-163 \mathrm{~A} \\
-163 \mathrm{~B} \\
-166 \mathrm{~A} \\
-166 \mathrm{~B} \\
-3 \mathrm{~B}-173 \mathrm{~A} \\
-173 \mathrm{~B} \\
-176 \mathrm{~A} \\
-176 \mathrm{~B}\end{array}$ & MLC, radial & $\begin{array}{l}4.79 \\
4.61 \\
4.70 \\
4.51 \\
4.16 \\
4.59 \\
4.60 \\
4.57\end{array}$ \\
\hline $\begin{array}{l}\text { Mean } \\
\text { Std. Dev. }\end{array}$ & 1 & $\begin{array}{l}4.57 \\
0.18\end{array}$ \\
\hline $\begin{array}{r}5651-74-3 \mathrm{~A}-152 \mathrm{~A} \\
-152 \mathrm{~B} \\
-154 \mathrm{~A} \\
-154 \mathrm{~B} \\
-3 \mathrm{~B}-157 \mathrm{~A} \\
-157 \mathrm{~B} \\
-159 \mathrm{~A} \\
-159 \mathrm{~B}\end{array}$ & MLE, axial & $\begin{array}{l}4.33 \\
4.27 \\
4.28 \\
4.13 \\
4.22 \\
4.27 \\
4.34 \\
4.24\end{array}$ \\
\hline $\begin{array}{l}\text { Mean } \\
\text { Std. Dev. }\end{array}$ & 1 & $\begin{array}{l}4.26 \\
0.07\end{array}$ \\
\hline $\begin{array}{r}5651-74-3 \mathrm{~A}-181 \mathrm{~A} \\
-181 \mathrm{~B} \\
-183 \mathrm{~A} \\
-183 \mathrm{~B} \\
3 \mathrm{~B}-185 \mathrm{~A} \\
-185 \mathrm{~B} \\
-187 \mathrm{~A} \\
-187 \mathrm{~B}\end{array}$ & MLE, radial & $\begin{array}{l}4.66 \\
4.71 \\
4.75 \\
4.74 \\
4.52 \\
4.68 \\
4.41 \\
4.52\end{array}$ \\
\hline $\begin{array}{l}\text { Mean } \\
\text { Std. Dev. }\end{array}$ & 1 & $\begin{array}{l}4.62 \\
0.12\end{array}$ \\
\hline
\end{tabular}

(a) Measured at UCC. 
TABLE $B-28$

THERMAL EXPANSIVITY OF TS-1240, LOT 1

[Log 5651-75 (UCC 46), whole $\log$ density $=1.84 \mathrm{~g} / \mathrm{cm}^{3(a)}$ ]

\begin{tabular}{|c|c|c|}
\hline $\begin{array}{l}\text { GA } \\
\text { Specimen } \\
\text { No. }\end{array}$ & $\begin{array}{l}\text { Location in Log } \\
\text { and Orientation }\end{array}$ & $\begin{array}{c}\text { Mean Coefficient of Thermal } \\
\text { Expansion, } \alpha \times 16^{\circ} \mathrm{C}^{-1} \\
\left(22^{\circ}-500^{\circ} \mathrm{C}\right)\end{array}$ \\
\hline $\begin{array}{r}5651-75-1 \mathrm{~A}-052 \mathrm{~A} \\
-052 \mathrm{~B} \\
-054 \mathrm{~A} \\
-054 \mathrm{~B} \\
-1 \mathrm{~B}-057 \mathrm{~A} \\
-057 \mathrm{~B} \\
-059 \mathrm{~A} \\
-059 \mathrm{~B}\end{array}$ & EE, axial & $\begin{array}{l}4.59 \\
4.38 \\
4.40 \\
4.27 \\
4.28 \\
4.42 \\
4.69 \\
4.56 \\
\end{array}$ \\
\hline $\begin{array}{l}\text { Mean } \\
\text { Std. Dev. }\end{array}$ & 1 & $\begin{array}{l}4.44 \\
0.151\end{array}$ \\
\hline $\begin{array}{r}5651-75-1 \mathrm{~A}-081 \mathrm{~A} \\
-081 \mathrm{~B} \\
-083 \mathrm{~A} \\
-083 \mathrm{~B} \\
-1 \mathrm{~B}-085 \mathrm{~A} \\
-085 \mathrm{~B} \\
-087 \mathrm{~A} \\
-087 \mathrm{~B}\end{array}$ & $E E$, radial & $\begin{array}{l}5.11 \\
4.80 \\
5.26 \\
4.81 \\
4.91 \\
4.86 \\
4.84 \\
5.03 \\
\end{array}$ \\
\hline $\begin{array}{l}\text { Mean } \\
\text { Std. Dev. }\end{array}$ & 1 & $\begin{array}{l}4.95 \\
0.166\end{array}$ \\
\hline $\begin{array}{r}5651-75-3 \mathrm{~A}-152 \mathrm{~A} \\
-152 \mathrm{~B} \\
-154 \mathrm{~A} \\
-154 \mathrm{~B} \\
-3 \mathrm{~B}-157 \mathrm{~A} \\
-157 \mathrm{~B} \\
-159 \mathrm{~A} \\
-159 \mathrm{~B}\end{array}$ & MLE, axial & $\begin{array}{l}4.21 \\
4.31 \\
4.20 \\
4.30 \\
3.94 \\
4.09 \\
4.31 \\
4.12 \\
\end{array}$ \\
\hline $\begin{array}{l}\text { Mean } \\
\text { Std. Dev. }\end{array}$ & 1 & $\begin{array}{l}4.18 \\
0.130\end{array}$ \\
\hline $\begin{array}{r}5651-75-3 \mathrm{~A}-181 \mathrm{~A} \\
-181 \mathrm{~B} \\
-183 \mathrm{~A} \\
-183 \mathrm{~B} \\
-3 \mathrm{~B}-185 \mathrm{~A} \\
-185 \mathrm{~B} \\
-187 \mathrm{~A} \\
-187 \mathrm{~B}\end{array}$ & MLE, radial & $\begin{array}{l}4.68 \\
4.67 \\
2.58 \\
4.72 \\
4.72 \\
4.73 \\
4.57 \\
4.94 \\
\end{array}$ \\
\hline $\begin{array}{l}\text { Mean } \\
\text { Std. Dev. }\end{array}$ & 1 & $\begin{array}{l}4.70 \\
0.114\end{array}$ \\
\hline
\end{tabular}


TABLE B-28 (Continued)

\begin{tabular}{|c|c|c|}
\hline $\begin{array}{l}\text { GA } \\
\text { Specimen } \\
\text { No. }\end{array}$ & $\begin{array}{l}\text { Location in Log } \\
\text { And Orientation }\end{array}$ & $\begin{array}{l}\text { Mean Coefficient of Thermal } \\
\text { Expansion, } \alpha \times 106^{\circ} \mathrm{C}^{-1} \\
\left(22^{\circ}-500^{\circ} \mathrm{C}\right)\end{array}$ \\
\hline $\begin{array}{r}5651-75-3 \mathrm{~A}-107 \mathrm{~A} \\
-107 \mathrm{~B} \\
-110 \mathrm{~A} \\
-110 \mathrm{~B} \\
-3 \mathrm{~B}-132 \mathrm{~A} \\
-132 \mathrm{~B} \\
-135 \mathrm{~A} \\
-135 \mathrm{~B}\end{array}$ & MLC, axial & $\begin{array}{l}3.87 \\
3.95 \\
3.86 \\
3.86 \\
3.96 \\
3.73 \\
4.03 \\
3.91\end{array}$ \\
\hline $\begin{array}{l}\text { Mean } \\
\text { Std. Dev. }\end{array}$ & 1 & $\begin{array}{l}3.90 \\
0.089\end{array}$ \\
\hline $\begin{array}{r}5651-75-3 \mathrm{~A}-163 \mathrm{~A} \\
-163 \mathrm{~B} \\
-166 \mathrm{~A} \\
-166 \mathrm{~B} \\
-3 \mathrm{~B}-173 \mathrm{~A} \\
-173 \mathrm{~B} \\
-176 \mathrm{~A} \\
-176 \mathrm{~B}\end{array}$ & MLC, radial & $\begin{array}{l}4.41 \\
4.33 \\
4.12 \\
4.31 \\
4.26 \\
4.42 \\
4.61 \\
4.45\end{array}$ \\
\hline $\begin{array}{l}\text { Mean } \\
\text { Std. Dev. }\end{array}$ & $\eta$ & $\begin{array}{l}4.36 \\
0.145\end{array}$ \\
\hline $\begin{array}{r}5651-75-1 \mathrm{~A}-007 \mathrm{~A} \\
-007 \mathrm{~B} \\
-010 \mathrm{~A} \\
-010 \mathrm{~B} \\
-1 \mathrm{~B}-032 \mathrm{~A} \\
-032 \mathrm{~B} \\
-035 \mathrm{~A} \\
-035 \mathrm{~B}\end{array}$ & $\mathrm{EC}$, axial & $\begin{array}{l}3.75 \\
3.58 \\
3.54 \\
3.63 \\
3.60 \\
3.61 \\
3.83 \\
3.99 \\
\end{array}$ \\
\hline $\begin{array}{l}\text { Mean } \\
\text { Std. Dev. }\end{array}$ & 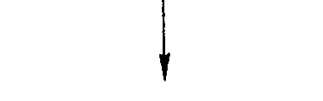 & $\begin{array}{l}3.69 \\
0.154\end{array}$ \\
\hline $\begin{array}{r}5651-75-1 \mathrm{~A}-063 \mathrm{~A} \\
-063 \mathrm{~B} \\
-066 \mathrm{~A} \\
-066 \mathrm{~B} \\
-1 \mathrm{~B}-073 \mathrm{~A} \\
-073 \mathrm{~B} \\
-076 \mathrm{~A} \\
-976 \mathrm{~B}\end{array}$ & $E C$, radial & $\begin{array}{l}4.20 \\
4.30 \\
4.17 \\
4.54 \\
4.30 \\
4.46 \\
4.29 \\
3.91\end{array}$ \\
\hline $\begin{array}{l}\text { Mean } \\
\text { Std. Dev. }\end{array}$ & 1 & $\begin{array}{l}4.27 \\
0.191\end{array}$ \\
\hline
\end{tabular}

(a) Measured at UCC. 
TABLE B-29

THERMAI EXPANSIVITY OF TS-1240, LOT 1

[ $\log$ 6484-29 (UCC 8-1S-ID-23), whole $\log$ density $=1.83 \mathrm{~g} / \mathrm{cm}^{3}{ }^{(a)}$ ]

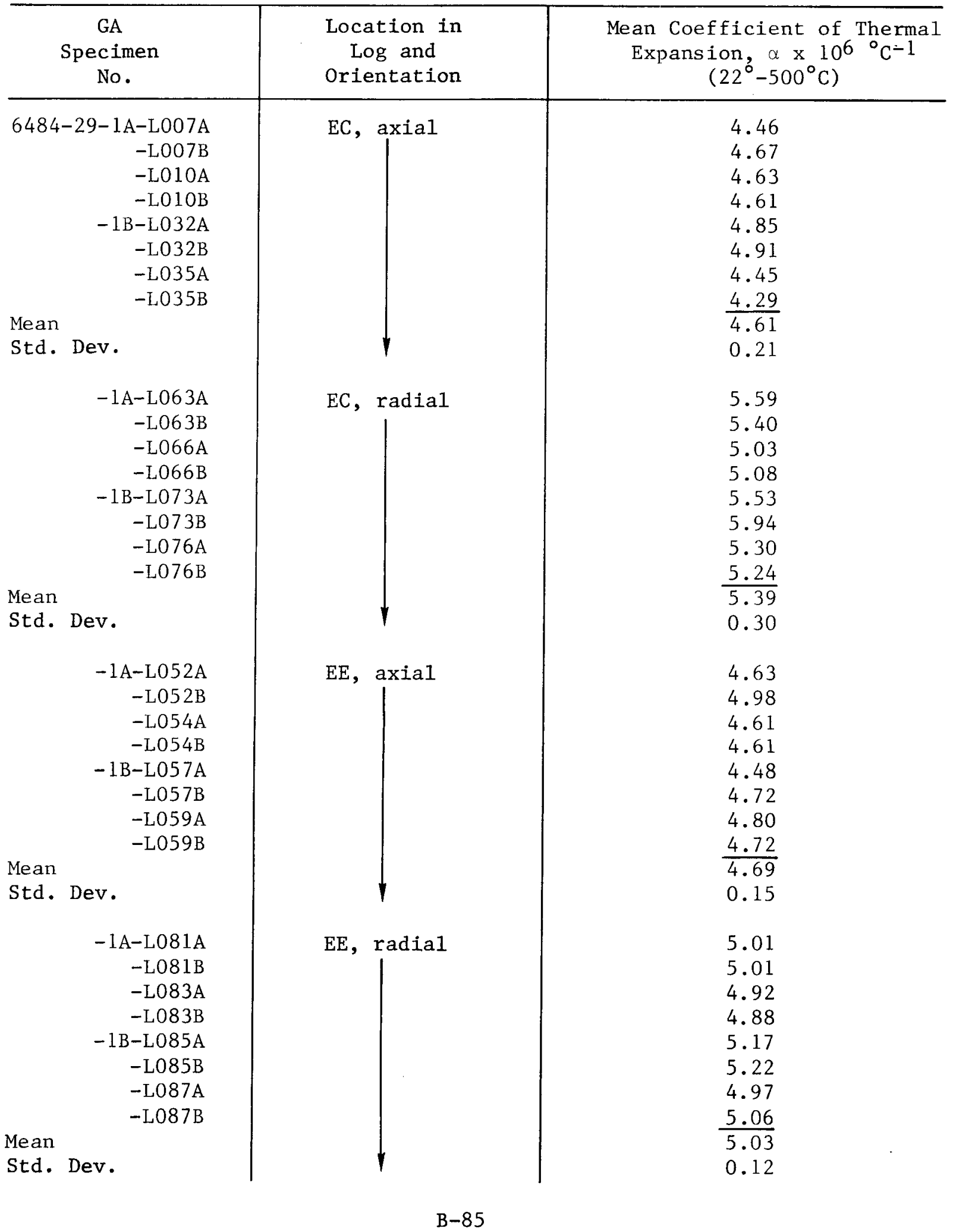


TABLE B-29 (Continued)

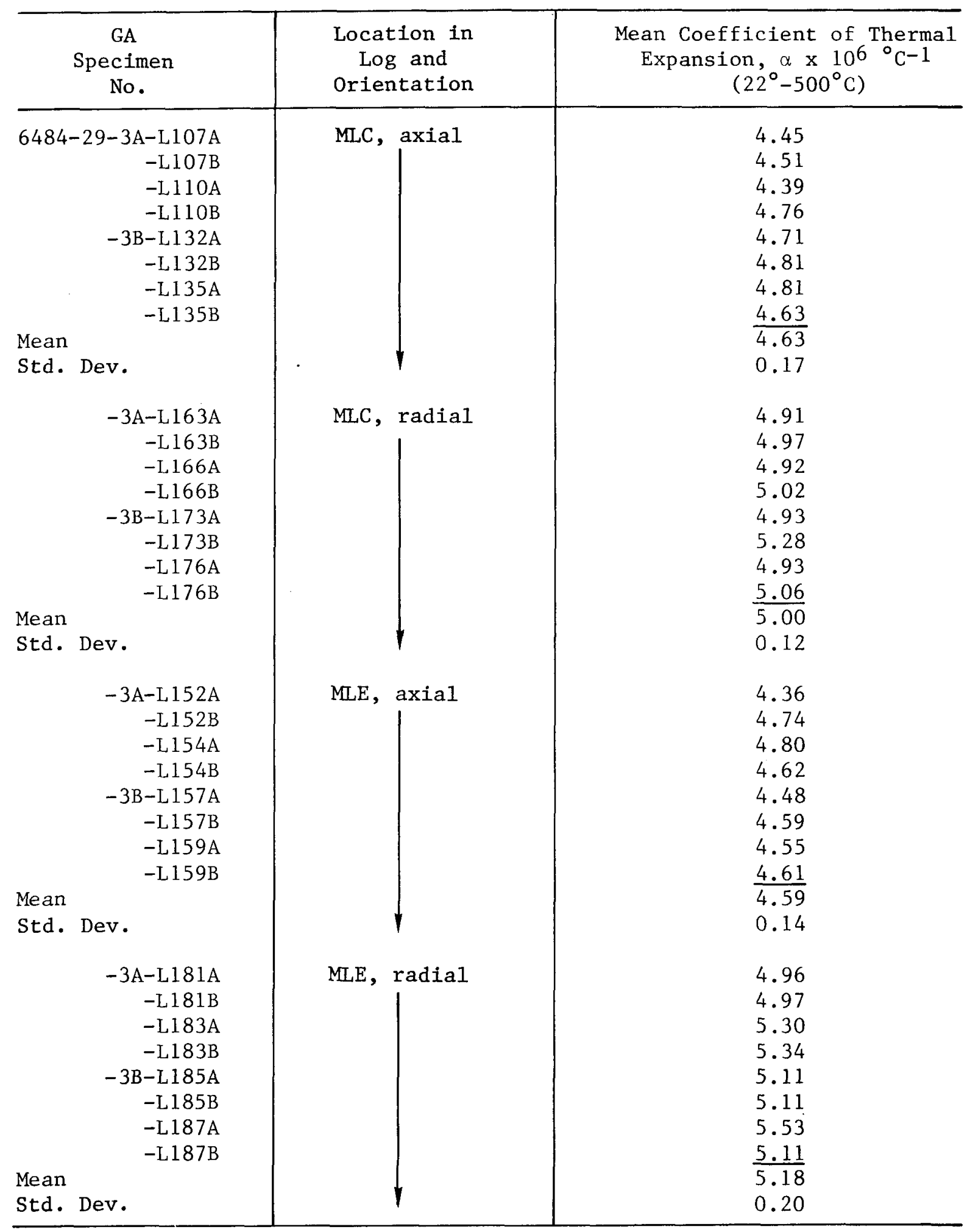

(a) Measured at UCC. 
TABLE B-30

THERMAL CONDUCTIVITY OF H-451, LOT 266

[Log 5651-28 (GLCC 18), whole log density $=1.77 \mathrm{~g} / \mathrm{cm}^{3(\mathrm{a})}$ ]

\begin{tabular}{|c|c|c|c|c|c|c|}
\hline \multirow{2}{*}{$\begin{array}{c}\text { GA } \\
\text { Specimen } \\
\text { No. }\end{array}$} & \multirow{2}{*}{$\begin{array}{l}\text { Location in } \\
\text { Log and } \\
\text { Orientation }\end{array}$} & \multicolumn{5}{|c|}{ Thermal Conductivity $\left(\mathrm{cal} / \mathrm{cm}-\mathrm{sec}-{ }^{\circ} \mathrm{C}\right)$} \\
\hline & & $22^{\circ} \mathrm{C}$ & $200^{\circ} \mathrm{C}$ & $400^{\circ} \mathrm{C}$ & $600^{\circ} \mathrm{C}$ & $800^{\circ} \mathrm{C}$ \\
\hline $\begin{array}{r}\mathrm{L}-0011 \\
0012 \\
0013 \\
0014 \\
0015 \\
0017 \\
0028 \\
0029 \\
0030 \\
0031 \\
0033 \\
0034\end{array}$ & MLC, radial & $\begin{array}{l}0.302 \\
0.324 \\
0.315 \\
0.317 \\
0.310 \\
0.287 \\
0.283 \\
0.278 \\
0.274 \\
0.270 \\
0.295 \\
0.285 \\
\end{array}$ & $\begin{array}{l}0.249 \\
0.273 \\
0.277 \\
0.275 \\
0.270 \\
0.245 \\
0.265 \\
0.265 \\
0.245 \\
0.261 \\
0.264 \\
0.260\end{array}$ & $\begin{array}{l}0.210 \\
0.235 \\
0.225 \\
0.223 \\
0.235 \\
0.204 \\
0.225 \\
0.226 \\
0.212 \\
0.223 \\
0.224 \\
0.222\end{array}$ & $\begin{array}{l}0.173 \\
0.195 \\
0.186 \\
0.175 \\
0.193 \\
0.173 \\
0.190 \\
0.198 \\
0.187 \\
0.185 \\
0.188 \\
0.175\end{array}$ & $\begin{array}{l}0.147 \\
0.163 \\
0.160 \\
0.145 \\
0.175 \\
0.150 \\
0.155 \\
0.165 \\
0.155 \\
0.162 \\
0.162 \\
0.154\end{array}$ \\
\hline $\begin{array}{l}\text { Mean } \\
\text { Std. Dev. }\end{array}$ & † & $\begin{array}{l}0.295 \\
0.018\end{array}$ & $\begin{array}{l}0.262 \\
.0 .011\end{array}$ & $\begin{array}{l}0.222 \\
0.009\end{array}$ & $\begin{array}{l}0.185 \\
0.009\end{array}$ & $\begin{array}{l}0.158 \\
0.008\end{array}$ \\
\hline $\begin{array}{r}\mathrm{L}-0137 \\
0138 \\
0139 \\
0140 \\
0141 \\
0142 \\
0143 \\
0144 \\
0145 \\
0146 \\
0147 \\
0148\end{array}$ & MLC, axial & $\begin{array}{l}0.355 \\
0.346 \\
0.344 \\
0.345 \\
0.347 \\
0.346 \\
0.351 \\
0.343 \\
0.345 \\
0.345 \\
0.346 \\
0.348 \\
\end{array}$ & $\begin{array}{l}0.308 \\
0.309 \\
0.306 \\
0.307 \\
0.300 \\
0.305 \\
0.303 \\
0.297 \\
0.298 \\
0.291 \\
0.300 \\
0.301 \\
\end{array}$ & $\begin{array}{l}0.220 \\
0.252 \\
0.250 \\
0.257 \\
0.253 \\
0.264 \\
0.244 \\
0.257 \\
0.251 \\
0.232 \\
0.241 \\
0.232 \\
\end{array}$ & $\begin{array}{l}0.204 \\
0.209 \\
0.217 \\
0.210 \\
0.209 \\
0.205 \\
0.207 \\
0.210 \\
0.203 \\
0.198 \\
0.198 \\
0.203 \\
\end{array}$ & $\begin{array}{l}0.166 \\
0.179 \\
0.188 \\
0.174 \\
0.175 \\
0.173 \\
0.168 \\
0.173 \\
0.174 \\
0.172 \\
0.173 \\
0.171 \\
\end{array}$ \\
\hline $\begin{array}{l}\text { Mean } \\
\text { Std. Dev. }\end{array}$ & 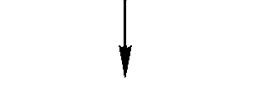 & $\begin{array}{l}0.347 \\
0.003\end{array}$ & $\begin{array}{l}0.302 \\
0.005\end{array}$ & $\begin{array}{l}0.246 \\
0.013\end{array}$ & $\begin{array}{l}0.206 \\
0.005\end{array}$ & $\begin{array}{l}0.174 \\
0.006\end{array}$ \\
\hline
\end{tabular}

(a) Measured at GLCC. 
TABLE B-31

THERMAL CONDUCTIVITY OF H-451, LOT 408

$\left[\log 5651-86\right.$ (GLCC 52), whole $\log$ density $\left.=1.74 \mathrm{gm} / \mathrm{cm}^{3}{ }^{(a)}\right]$

\begin{tabular}{|c|c|c|c|c|c|c|}
\hline \multirow{2}{*}{$\begin{array}{c}\text { GA } \\
\text { Specimen } \\
\text { No. }\end{array}$} & \multirow{2}{*}{$\begin{array}{l}\text { Location in } \\
\text { Log and } \\
\text { Orientation }\end{array}$} & \multicolumn{5}{|c|}{ Thermal Conductivity $\left(\mathrm{ca} 1 / \mathrm{cm}-\mathrm{sec}-{ }^{\circ} \mathrm{C}\right)$} \\
\hline & & $22^{\circ} \mathrm{C}$ & $200^{\circ} \mathrm{C}$ & $400^{\circ} \mathrm{C}$ & $600^{\circ} \mathrm{C}$ & $800^{\circ} \mathrm{C}$ \\
\hline $\begin{array}{r}5651-86-3 A-L 1 M \\
-L 1 N \\
-L 10 \\
-L 1 P \\
-L 1 Q \\
-L 1 R \\
-L 1 S \\
-L 1 T\end{array}$ & MLC, axial & $\begin{array}{l}0.288 \\
0.252 \\
0.242 \\
0.244 \\
0.253 \\
0.253 \\
0.242 \\
0.248\end{array}$ & $\begin{array}{l}0.273 \\
0.245 \\
0.238 \\
0.236 \\
0.235 \\
0.219 \\
0.215 \\
0.246\end{array}$ & $\begin{array}{l}0.242 \\
0.218 \\
0.205 \\
0.203 \\
0.203 \\
0.182 \\
0.195 \\
0.209\end{array}$ & $\begin{array}{l}0.196 \\
0.183 \\
0.176 \\
0.174 \\
0.174 \\
0.163 \\
0.173 \\
0.173\end{array}$ & $\begin{array}{l}0.177 \\
0.141 \\
0.150 \\
0.155 \\
0.155 \\
0.144 \\
0.147 \\
0.151\end{array}$ \\
\hline $\begin{array}{l}\text { Mean } \\
\text { Std. Dev. }\end{array}$ & 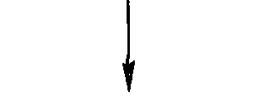 & $\begin{array}{l}0.253 \\
0.015\end{array}$ & $\begin{array}{l}0.238 \\
0.018\end{array}$ & $\begin{array}{l}0.207 \\
0.017\end{array}$ & $\begin{array}{l}0.176 \\
0.010\end{array}$ & $\begin{array}{l}0.152 \\
0.011\end{array}$ \\
\hline $\begin{aligned} 5651-86-3 A-L 8 A \\
-L 8 B \\
-L 8 C \\
-L 8 D \\
-L 8 E \\
-L 8 F \\
-L 8 G \\
-L 8 H\end{aligned}$ & MLC, radial & $\begin{array}{l}0.249 \\
0.253 \\
0.242 \\
0.253 \\
0.266 \\
0.247 \\
0.251 \\
0.248\end{array}$ & $\begin{array}{l}0.221 \\
0.216 \\
0.234 \\
0.233 \\
0.236 \\
0.233 \\
0.228 \\
0.224\end{array}$ & $\begin{array}{l}0.188 \\
0.190 \\
0.201 \\
0.203 \\
0.210 \\
0.199 \\
0.192 \\
0.189\end{array}$ & $\begin{array}{l}0.160 \\
0.156 \\
0.173 \\
0.169 \\
0.178 \\
0.166 \\
0.166 \\
0.164\end{array}$ & $\begin{array}{l}0.145 \\
0.133 \\
0.144 \\
0.148 \\
0.152 \\
0.150 \\
0.147 \\
0.141\end{array}$ \\
\hline $\begin{array}{l}\text { Mean } \\
\text { Std. Dev. }\end{array}$ & 1 & $\begin{array}{l}0.251 \\
0.007\end{array}$ & $\begin{array}{l}0.228 \\
0.007\end{array}$ & $\begin{array}{l}0.196 \\
0.008\end{array}$ & $\begin{array}{l}0.166 \\
0.007\end{array}$ & $\begin{array}{l}0.145 \\
0.006\end{array}$ \\
\hline
\end{tabular}

(a) Measured at GLCC. 
TABLE B-32

THERMAL CONDUCTIVITY OF H-451, LOT 426

[ Log 6484-34 (GLCC 198), whole $\log$ density $=1.73 \mathrm{~g} / \mathrm{cm}^{3}{ }^{(\mathrm{a})}$ and $\log 6484-41$ (GLCC 184), whole $\log$ density $\left.=1.72 \mathrm{~g} / \mathrm{cm}^{3}(\mathrm{a})\right]$

\begin{tabular}{|c|c|c|c|c|c|c|}
\hline \multirow{2}{*}{$\begin{array}{c}\text { GA } \\
\text { Specimen } \\
\text { No. }\end{array}$} & \multirow{2}{*}{$\begin{array}{l}\text { Location in } \\
\text { Log and } \\
\text { Orientation }\end{array}$} & \multicolumn{5}{|c|}{ Thermal Conductivity $\left(\mathrm{ca} 1 / \mathrm{cm}-\mathrm{sec}-{ }^{\circ} \mathrm{C}\right)$} \\
\hline & & $22^{\circ} \mathrm{C}$ & $200^{\circ} \mathrm{C}$ & $400^{\circ} \mathrm{C}$ & $600^{\circ} \mathrm{C}$ & $800^{\circ} \mathrm{C}$ \\
\hline $\begin{array}{r}6484-34-3 \mathrm{~A}-\mathrm{L} 51 \mathrm{~A} \\
-\mathrm{L} 51 \mathrm{~B} \\
-\mathrm{L} 55 \mathrm{~A} \\
-\mathrm{L} 55 \mathrm{~B} \\
-3 \mathrm{~B}-\mathrm{L} 62 \mathrm{~A} \\
-\mathrm{L} 62 \mathrm{~B} \\
-\mathrm{L} 66 \mathrm{~A} \\
-\mathrm{L} 66 \mathrm{~B}\end{array}$ & MLC, axial & $\begin{array}{l}0.271 \\
0.327 \\
0.315 \\
0.317 \\
0.281 \\
0.382 \\
0.384 \\
0.323 \\
\end{array}$ & $\begin{array}{l}0.227 \\
0.268 \\
0.292 \\
0.266 \\
0.271 \\
0.442 \\
0.275 \\
0.265 \\
\end{array}$ & $\begin{array}{l}0.186 \\
0.211 \\
0.241 \\
0.228 \\
0.224 \\
0.231 \\
0.224 \\
0.225 \\
\end{array}$ & $\begin{array}{l}0.164 \\
0.162 \\
0.190 \\
0.192 \\
0.182 \\
0.180 \\
0.187 \\
0.174 \\
\end{array}$ & $\begin{array}{l}0.138 \\
0.147 \\
0.165 \\
0.163 \\
0.158 \\
0.163 \\
0.167 \\
0.142 \\
\end{array}$ \\
\hline $\begin{array}{l}\text { Mean } \\
\text { Std. Dev. }\end{array}$ & 1 & $\begin{array}{l}0.325 \\
0.041\end{array}$ & $\begin{array}{l}0.288 \\
0.065\end{array}$ & $\begin{array}{l}0.221 \\
0.017\end{array}$ & $\begin{array}{l}0.179 \\
0.011\end{array}$ & $\begin{array}{l}0.155 \\
0.011\end{array}$ \\
\hline $\begin{array}{r}6484-34-3 A-L 78 A \\
-L 78 B \\
-L 81 A \\
-L 81 B \\
-3 B-L 90 A \\
-L 90 B \\
-L 93 A \\
-L 93 B\end{array}$ & MLC, radial & $\begin{array}{l}0.312 \\
0.310 \\
0.341 \\
0.324 \\
0.258 \\
0.308 \\
0.312 \\
0.296 \\
\end{array}$ & $\begin{array}{l}0.270 \\
0.273 \\
0.303 \\
0.266 \\
0.284 \\
0.259 \\
0.282 \\
0.266 \\
\end{array}$ & $\begin{array}{l}0.201 \\
0.200 \\
0.222 \\
0.197 \\
0.235 \\
0.199 \\
0.205 \\
0.226 \\
\end{array}$ & $\begin{array}{l}0.173 \\
0.144 \\
0.189 \\
0.175 \\
0.179 \\
0.170 \\
0.180 \\
0.192 \\
\end{array}$ & $\begin{array}{l}0.149 \\
0.141 \\
0.156 \\
0.136 \\
0.163 \\
0.142 \\
0.144 \\
0.168 \\
\end{array}$ \\
\hline $\begin{array}{l}\text { Mean } \\
\text { Std. Dev. }\end{array}$ & 1 & $\begin{array}{l}0.308 \\
0.024\end{array}$ & $\begin{array}{l}0.275 \\
0.014\end{array}$ & $\begin{array}{l}0.211 \\
0.015\end{array}$ & $\begin{array}{l}0.175 \\
0.015\end{array}$ & $\begin{array}{l}0.150 \\
0.011\end{array}$ \\
\hline $\begin{array}{r}6484-41-3 \mathrm{~A}-\mathrm{L} 003 \mathrm{~A} \\
-\mathrm{L} 003 \mathrm{~B} \\
-\mathrm{L} 004 \mathrm{~A} \\
-\mathrm{L} 004 \mathrm{~B} \\
-3 \mathrm{~B}-\mathrm{L} 003 \mathrm{~A} \\
-\mathrm{L} 003 \mathrm{~B} \\
-\mathrm{L} 004 \mathrm{~A}\end{array}$ & MLC, axial & $\begin{array}{l}0.325 \\
0.341 \\
0.430 \\
0.381 \\
0.339 \\
0.328 \\
0.312 \\
\end{array}$ & $\begin{array}{l}0.280 \\
0.272 \\
0.296 \\
0.366 \\
0.301 \\
0.306 \\
0.268 \\
\end{array}$ & $\begin{array}{l}0.219 \\
0.215 \\
0.203 \\
0.219 \\
0.224 \\
0.232 \\
0.228 \\
\end{array}$ & $\begin{array}{l}0.179 \\
0.157 \\
0.175 \\
0.190 \\
0.188 \\
0.198 \\
0.189 \\
\end{array}$ & $\begin{array}{l}0.151 \\
0.150 \\
0.148 \\
0.148 \\
0.167 \\
0.158 \\
0.157 \\
\end{array}$ \\
\hline $\begin{array}{l}\text { Mean } \\
\text { Std. Dev. }\end{array}$ & $\varphi$ & $\begin{array}{l}0.351 \\
0.041\end{array}$ & $\begin{array}{l}0.298 \\
0.033\end{array}$ & $\begin{array}{l}0.220 \\
0.009\end{array}$ & $\begin{array}{l}0.183 \\
0.013\end{array}$ & $\begin{array}{l}0.154 \\
0.007\end{array}$ \\
\hline $\begin{array}{r}6484-41-3 \mathrm{~A}-\mathrm{L} 016 \mathrm{~A} \\
-\mathrm{L} 016 \mathrm{~B} \\
-\mathrm{L} 022 \mathrm{~A} \\
-\mathrm{L} 022 \mathrm{~B} \\
-3 \mathrm{~B}-\mathrm{L} 016 \mathrm{~A} \\
-\mathrm{L} 016 \mathrm{~B} \\
-\mathrm{L} 022 \mathrm{~A}\end{array}$ & MLC, radial & $\begin{array}{l}0.308 \\
0.293 \\
0.340 \\
0.309 \\
0.382 \\
0.361 \\
0.336 \\
\end{array}$ & $\begin{array}{l}0.272 \\
0.245 \\
0.286 \\
0.272 \\
0.274 \\
0.281 \\
0.244 \\
\end{array}$ & $\begin{array}{l}0.205 \\
0.204 \\
0.221 \\
0.215 \\
0.216 \\
0.219 \\
0.197 \\
\end{array}$ & $\begin{array}{l}0.189 \\
0.159 \\
0.178 \\
0.174 \\
0.188 \\
0.179 \\
0.168 \\
\end{array}$ & $\begin{array}{l}0.150 \\
0.139 \\
0.138 \\
0.162 \\
0.163 \\
0.151 \\
0.145 \\
\end{array}$ \\
\hline $\begin{array}{l}\text { Mean } \\
\text { Std. Dev. }\end{array}$ & 1 & $\begin{array}{l}0.333 \\
0.032\end{array}$ & $\begin{array}{l}0.268 \\
0.017\end{array}$ & $\begin{array}{l}0.211 \\
0.009\end{array}$ & $\begin{array}{l}0.176 \\
0.011\end{array}$ & $\begin{array}{l}0.150 \\
0.010\end{array}$ \\
\hline
\end{tabular}

(a) Measured at GLCC. 
TABLE $B-33$

THERMAL CONDUCTIVITY OF TS- 1240 , LOT

[Log 5651-72 (UCC 32), whole log density $=1.77 \mathrm{~g} / \mathrm{cm}^{3}$ (a) and $\log 5651-75$ (UCC 46), whole $\log$ density $=1.84 \mathrm{~g} / \mathrm{cm}^{3}(\mathrm{a})$ ]

\begin{tabular}{|c|c|c|c|c|c|c|}
\hline \multirow{2}{*}{$\begin{array}{c}\text { GA } \\
\text { Specimen } \\
\text { No. }\end{array}$} & \multirow{2}{*}{$\begin{array}{l}\text { Position in } \\
\text { Log and } \\
\text { Orientation }\end{array}$} & \multicolumn{5}{|c|}{ Therma1 Conductivity $\left(\mathrm{cal} / \mathrm{cm}-\mathrm{sec}-{ }^{\circ} \mathrm{C}\right)$} \\
\hline & & $22^{\circ} \mathrm{C}$ & $200^{\circ} \mathrm{C}$ & $400^{\circ} \mathrm{C}$ & $600^{\circ} \mathrm{C}$ & $800^{\circ} \mathrm{C}$ \\
\hline $\begin{array}{r}5651-72-3 \mathrm{~A}-\mathrm{L} 51 \mathrm{~A} \\
-\mathrm{L} 51 \mathrm{~B} \\
-\mathrm{L} 55 \mathrm{~A} \\
-\mathrm{L} 55 \mathrm{~B} \\
-3 \mathrm{~B}-\mathrm{L} 62 \mathrm{~A} \\
-\mathrm{L} 62 \mathrm{~B} \\
-\mathrm{L} 66 \mathrm{~A} \\
-\mathrm{L} 66 \mathrm{~B}\end{array}$ & QLC, axial & $\begin{array}{l}0.234 \\
0.242 \\
0.265 \\
0.256 \\
0.224 \\
0.231 \\
0.266 \\
0.235 \\
\end{array}$ & $\begin{array}{l}0.222 \\
0.236 \\
0.237 \\
0.220 \\
0.207 \\
0.205 \\
0.232 \\
0.212\end{array}$ & $\begin{array}{l}0.196 \\
0.190 \\
0.193 \\
0.193 \\
0.183 \\
0.182 \\
0.213 \\
0.188\end{array}$ & $\begin{array}{l}0.153 \\
0.162 \\
0.163 \\
0.151 \\
0.152 \\
0.156 \\
0.169 \\
0.153\end{array}$ & $\begin{array}{l}0.130 \\
0.139 \\
0.139 \\
0.134 \\
0.133 \\
0.129 \\
0.151 \\
0.129\end{array}$ \\
\hline $\begin{array}{l}\text { Mean } \\
\text { Std. Dev. }\end{array}$ & 1 & $\begin{array}{l}0.245 \\
0.017\end{array}$ & $\begin{array}{l}0.222 \\
0.013\end{array}$ & $\begin{array}{l}0.193 \\
0.010\end{array}$ & $\begin{array}{l}0.157 \\
0.006\end{array}$ & $\begin{array}{l}0.136 \\
0.007\end{array}$ \\
\hline $\begin{array}{r}5651-72-3 \mathrm{~A}-\mathrm{L} 78 \mathrm{~A} \\
-\mathrm{L} 78 \mathrm{~B} \\
-\mathrm{L} 81 \mathrm{~A} \\
-\mathrm{L} 81 \mathrm{~B} \\
-3 \mathrm{~B}-\mathrm{L} 90 \mathrm{~A} \\
-\mathrm{L} 90 \mathrm{~B} \\
-\mathrm{L} 93 \mathrm{~A} \\
-\mathrm{L} 93 \mathrm{~B}\end{array}$ & QLC, radial & $\begin{array}{l}0.219 \\
0.243 \\
0.229 \\
0.247 \\
0.242 \\
0.248 \\
0.231 \\
0.222 \\
\end{array}$ & $\begin{array}{l}0.208 \\
0.230 \\
0.216 \\
0.219 \\
0.198 \\
0.216 \\
0.215 \\
0.201\end{array}$ & $\begin{array}{l}0.183 \\
0.193 \\
0.181 \\
0.186 \\
0.178 \\
0.184 \\
0.187 \\
0.170\end{array}$ & $\begin{array}{l}0.150 \\
0.170 \\
0.172 \\
0.150 \\
0.157 \\
0.165 \\
0.175 \\
0.145 \\
\end{array}$ & $\begin{array}{l}0.123 \\
0.146 \\
0.141 \\
0.130 \\
0.127 \\
0.139 \\
0.142 \\
0.125 \\
\end{array}$ \\
\hline $\begin{array}{l}\text { Mean } \\
\text { Std. Dev. }\end{array}$ & r & $\begin{array}{l}0.235 \\
0.011\end{array}$ & $\begin{array}{l}0.213 \\
0.010\end{array}$ & $\begin{array}{l}0.183 \\
0.007\end{array}$ & $\begin{array}{l}0.160 \\
0.011\end{array}$ & $\begin{array}{l}0.134 \\
0.009\end{array}$ \\
\hline $\begin{array}{r}5651-75-3 \mathrm{~A}-\mathrm{L} 51 \mathrm{~A} \\
-\mathrm{L} 51 \mathrm{~B} \\
-\mathrm{L} 55 \mathrm{~A} \\
-\mathrm{L} 55 \mathrm{~B} \\
-3 \mathrm{~B}-\mathrm{L} 62 \mathrm{~A} \\
-\mathrm{L} 62 \mathrm{~B} \\
-\mathrm{L} 66 \mathrm{~A} \\
-\mathrm{L} 66 \mathrm{~B}\end{array}$ & MLC, axial & $\begin{array}{l}0.323 \\
0.246 \\
0.255 \\
0.248 \\
0.240 \\
0.266 \\
0.262 \\
0.248 \\
\end{array}$ & $\begin{array}{l}0.278 \\
0.244 \\
0.250 \\
0.238 \\
0.233 \\
0.245 \\
0.237 \\
0.236\end{array}$ & $\begin{array}{l}0.240 \\
0.214 \\
0.222 \\
0.221 \\
0.217 \\
0.223 \\
0.220 \\
0.216\end{array}$ & $\begin{array}{l}0.210 \\
0.169 \\
0.194 \\
0.195 \\
0.184 \\
0.189 \\
0.192 \\
0.188 \\
\end{array}$ & $\begin{array}{l}0.175 \\
0.151 \\
0.161 \\
0.172 \\
0.157 \\
0.168 \\
0.163 \\
0.166 \\
\end{array}$ \\
\hline $\begin{array}{l}\text { Mean } \\
\text { Std. Dev. }\end{array}$ & $\varphi$ & $\begin{array}{l}0.261 \\
0.026\end{array}$ & $\begin{array}{l}0.245 \\
0.014\end{array}$ & $\begin{array}{l}0.222 \\
0.008\end{array}$ & $\begin{array}{l}0.190 \\
0.011\end{array}$ & $\begin{array}{l}0.164 \\
0.008\end{array}$ \\
\hline $\begin{array}{r}5651-75-3 \mathrm{~A}-\mathrm{L} 78 \mathrm{~A} \\
-\mathrm{L} 78 \mathrm{~B} \\
-\mathrm{L} 81 \mathrm{~A} \\
-\mathrm{L} 81 \mathrm{~B} \\
-3 \mathrm{~B}-\mathrm{L} 90 \mathrm{~A} \\
-\mathrm{L} 90 \mathrm{~B} \\
-\mathrm{L} 93 \mathrm{~A} \\
-\mathrm{L} 93 \mathrm{~B}\end{array}$ & MLC, radial & $\begin{array}{l}0.246 \\
0.254 \\
0.248 \\
0.262 \\
0.241 \\
0.243 \\
0.275 \\
0.265\end{array}$ & $\begin{array}{l}0.244 \\
0.249 \\
0.248 \\
0.244 \\
0.235 \\
0.241 \\
0.250 \\
0.255\end{array}$ & $\begin{array}{l}0.199 \\
0.213 \\
0.216 \\
0.204 \\
0.202 \\
0.211 \\
0.210 \\
0.214\end{array}$ & $\begin{array}{l}0.178 \\
0.188 \\
0.184 \\
0.177 \\
0.180 \\
0.186 \\
0.185 \\
0.189\end{array}$ & $\begin{array}{l}0.151 \\
0.164 \\
0.160 \\
0.156 \\
0.154 \\
0.160 \\
0.161 \\
0.165\end{array}$ \\
\hline $\begin{array}{l}\text { Mean } \\
\text { Std. Dev. }\end{array}$ & 1 & $\begin{array}{l}0.254 \\
0.012\end{array}$ & $\begin{array}{l}0.254 \\
0.006\end{array}$ & $\begin{array}{l}0.209 \\
0.006\end{array}$ & $\begin{array}{l}0.183 \\
0.004\end{array}$ & $\begin{array}{l}0.159 \\
0.005\end{array}$ \\
\hline
\end{tabular}

(a) Measured at UCC. 
TABLE B-34

THERMAL CONDUCTIVITY OF TS-1240, LOT 1

[ $\log 5651-73$ (UCC 40), whole log density $=1.80 \mathrm{~g} / \mathrm{cm}^{3}{ }^{(a)}$ ]

\begin{tabular}{|c|c|c|c|c|c|c|}
\hline \multirow{2}{*}{$\begin{array}{c}\text { GA } \\
\text { Specimen } \\
\text { No. } \\
\end{array}$} & \multirow{2}{*}{$\begin{array}{l}\text { Location in } \\
\text { Log and } \\
\text { Orientation }\end{array}$} & \multicolumn{5}{|c|}{ Thermal Conductivity $\left(\mathrm{cal} / \mathrm{cm}-\mathrm{sec}-{ }^{\circ} \mathrm{C}\right)$} \\
\hline & & $22^{\circ} \mathrm{C}$ & $200^{\circ} \mathrm{C}$ & $400^{\circ} \mathrm{C}$ & $600^{\circ} \mathrm{C}$ & $800^{\circ} \mathrm{C}$ \\
\hline $\begin{array}{r}5651-73-3 \mathrm{~A}-\mathrm{L} 001 \mathrm{M} \\
-\mathrm{L} 001 \mathrm{~N} \\
-\mathrm{L} 0010 \\
-\mathrm{L} 001 \mathrm{P} \\
-\mathrm{L} 001 \mathrm{Q} \\
-\mathrm{L} 001 \mathrm{R} \\
-\mathrm{L} 001 \mathrm{~S} \\
-\mathrm{L} 001 \mathrm{~T}\end{array}$ & MLC, axial & $\begin{array}{l}0.211 \\
0.219 \\
0.226 \\
0.230 \\
0.225 \\
0.245 \\
0.274 \\
0.243 \\
\end{array}$ & $\begin{array}{l}0.204 \\
0.216 \\
0.213 \\
0.221 \\
0.221 \\
0.229 \\
0.250 \\
0.233 \\
\end{array}$ & $\begin{array}{l}0.185 \\
0.174 \\
0.180 \\
0.199 \\
0.188 \\
0.192 \\
0.218 \\
0.204 \\
\end{array}$ & $\begin{array}{l}0.159 \\
0.155 \\
0.160 \\
0.164 \\
0.165 \\
0.163 \\
0.176 \\
0.180 \\
\end{array}$ & $\begin{array}{l}0.139 \\
0.139 \\
0.143 \\
0.143 \\
0.144 \\
0.139 \\
0.154 \\
0.157 \\
\end{array}$ \\
\hline $\begin{array}{l}\text { Mean } \\
\text { Std. Dev. }\end{array}$ & 1 & $\begin{array}{l}0.234 \\
0.020\end{array}$ & $\begin{array}{l}0.223 \\
0.014\end{array}$ & $\begin{array}{l}0.192 \\
0.014\end{array}$ & $\begin{array}{l}0.165 \\
0.008\end{array}$ & $\begin{array}{l}0.145 \\
0.007\end{array}$ \\
\hline $\begin{array}{r}5651-73-3 A-L 008 \mathrm{~A} \\
-\mathrm{L} 008 \mathrm{~B} \\
-\mathrm{L} 008 \mathrm{C} \\
-\mathrm{L} 008 \mathrm{D} \\
-\mathrm{L} 008 \mathrm{E} \\
-\mathrm{L} 008 \mathrm{~F} \\
-\mathrm{L} 008 \mathrm{G} \\
-\mathrm{L} 008 \mathrm{H}\end{array}$ & MLC, radial & $\begin{array}{l}0.242 \\
0.244 \\
0.258 \\
0.230 \\
0.260 \\
0.251 \\
0.246 \\
0.249 \\
\end{array}$ & $\begin{array}{l}0.230 \\
0.233 \\
0.246 \\
0.224 \\
0.230 \\
0.231 \\
0.227 \\
0.229 \\
\end{array}$ & $\begin{array}{l}0.203 \\
0.188 \\
0.202 \\
0.184 \\
0.207 \\
0.190 \\
0.193 \\
0.197 \\
\end{array}$ & $\begin{array}{l}0.166 \\
0.161 \\
0.179 \\
0.161 \\
0.175 \\
0.163 \\
0.168 \\
0.166 \\
\end{array}$ & $\begin{array}{l}0.158 \\
0.144 \\
0.154 \\
0.141 \\
0.151 \\
0.143 \\
0.143 \\
0.147 \\
\end{array}$ \\
\hline $\begin{array}{l}\text { Mean } \\
\text { Std. Dev. }\end{array}$ & 1 & $\begin{array}{l}0.247 \\
0.010\end{array}$ & $\begin{array}{l}0.231 \\
0.006\end{array}$ & $\begin{array}{l}0.195 \\
0.008\end{array}$ & $\begin{array}{l}0.167 \\
0.006\end{array}$ & $\begin{array}{l}0.148 \\
0.006\end{array}$ \\
\hline
\end{tabular}

(a) Measured at UCC. 
TABLE B-35

IMPURITY CONTENT OF H-451

[in $\operatorname{ppm}(\mathrm{a})]$

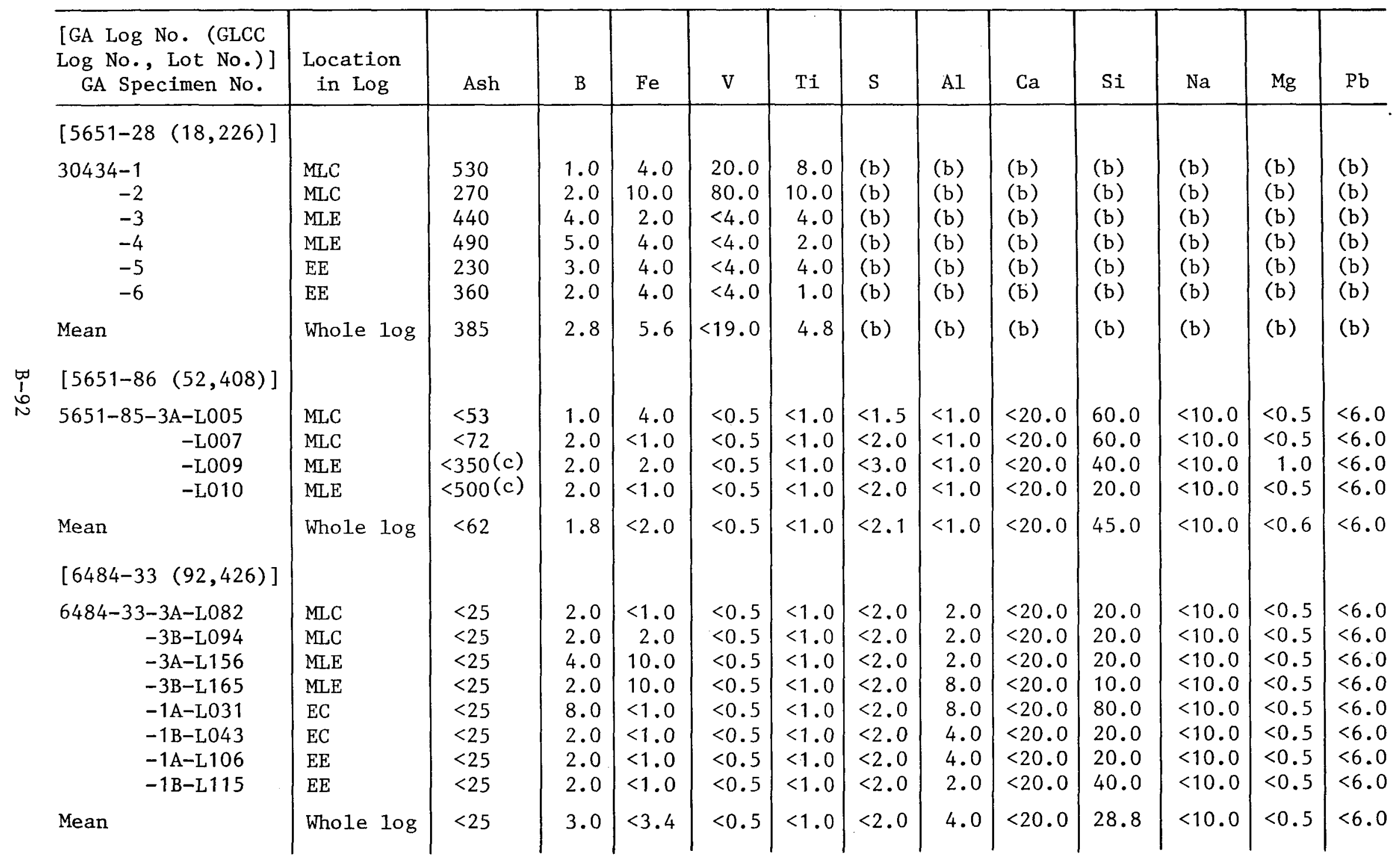


TABLE B-35 (Continued)

\begin{tabular}{|c|c|c|c|c|c|c|c|c|c|c|c|c|c|}
\hline $\begin{array}{l}\text { [GA Log No. (GLCC } \\
\text { Log No., Lot No.)] } \\
\text { GA Specimen No. }\end{array}$ & $\begin{array}{l}\text { Location } \\
\text { in Log }\end{array}$ & Ash & B & $\mathrm{Fe}$ & V & $\mathrm{Ti}$ & $\mathrm{S}$ & A1 & $\mathrm{Ca}$ & $\mathrm{Si}$ & $\mathrm{Na}$ & $\mathrm{Mg}$ & $\mathrm{Pb}$ \\
\hline $\begin{array}{r}6484-34-3 A-L 082 \\
-3 B-L 094 \\
-3 A-L 156 \\
-3 B-L 165 \\
-1 A-L 031 \\
-1 B-L 043 \\
-1 A-L 106 \\
-1 B-L 115\end{array}$ & $\begin{array}{l}\text { MLC } \\
\text { MLC } \\
\text { MLE } \\
\text { MLE } \\
\text { EC } \\
E C \\
\text { EE } \\
\text { EE }\end{array}$ & $\begin{array}{l}<25 \\
<25 \\
<25 \\
<25 \\
<25 \\
<25 \\
<25 \\
<25\end{array}$ & $\begin{array}{r}20.0 \\
2.0 \\
2.0 \\
2.0 \\
2.0 \\
4.0 \\
2.0 \\
4.0\end{array}$ & $\begin{array}{r}<1.0 \\
<1.0 \\
<1.0 \\
<1.0 \\
10.0 \\
40.0 \\
<1.0 \\
4.0\end{array}$ & $\begin{array}{l}<0.5 \\
<0.5 \\
<0.5 \\
<0.5 \\
<0.5 \\
<0.5 \\
<0.5 \\
<0.5\end{array}$ & $\begin{array}{l}<1.0 \\
<1.0 \\
<1.0 \\
<1.0 \\
<1.0 \\
10.0 \\
<1.0 \\
<1.0\end{array}$ & $\begin{array}{l}<2.0 \\
<3.0 \\
<2.0 \\
<2.0 \\
<2.0 \\
<2.0 \\
<2.0 \\
<2.0\end{array}$ & $\begin{array}{r}4.0 \\
4.0 \\
4.0 \\
10.0 \\
8.0 \\
10.0 \\
10.0 \\
40.0\end{array}$ & $\begin{array}{r}<20.0 \\
<20.0 \\
<20.0 \\
<20.0 \\
<20.0 \\
80.0 \\
<20.0 \\
<20.0\end{array}$ & $\begin{array}{l}20.0 \\
10.0 \\
40.0 \\
60.0 \\
40.0 \\
60.0 \\
20.0 \\
40.0\end{array}$ & $\begin{array}{r}<10.0 \\
20.0 \\
<10.0 \\
<10.0 \\
<10.0 \\
<10.0 \\
10.0 \\
<10.0\end{array}$ & $\begin{array}{r}<0.5 \\
<0.5 \\
<0.5 \\
<0.5 \\
<0.5 \\
<0.5 \\
1.0 \\
1.0\end{array}$ & $\begin{array}{l}<6.0 \\
<6.0 \\
<6.0 \\
<6.0 \\
<6.0 \\
40.0 \\
<6.0 \\
<6.0\end{array}$ \\
\hline $\begin{array}{l}\text { Mean } \\
{[6484-40(155,426)]}\end{array}$ & Whole $\log$ & $<25$ & 4.8 & $<7.4$ & $<0.5$ & $<2.1$ & $<2.1$ & 11.3 & $<27.5$ & 36.3 & $<11.3$ & $<0.6$ & $<10.3$ \\
\hline $\begin{array}{r}6484-40-3 \mathrm{~A}-\mathrm{L} 016 \\
-3 \mathrm{~B}-\mathrm{L} 016 \\
-3 \mathrm{~A}-\mathrm{L} 036 \\
-3 \mathrm{~B}-\mathrm{L} 036 \\
-1 \mathrm{~A}-\mathrm{L} 016 \\
-1 \mathrm{~B}-\mathrm{L} 016 \\
-1 \mathrm{~A}-\mathrm{L} 036 \\
-1 \mathrm{~B}-\mathrm{L} 036\end{array}$ & $\begin{array}{l}\text { MLC } \\
\text { MLC } \\
\text { MLE } \\
\text { MLE } \\
E C \\
E C \\
E E \\
E E\end{array}$ & $\begin{array}{r}110 \\
60 \\
140 \\
70 \\
90 \\
50 \\
70 \\
120\end{array}$ & $\begin{array}{r}1.0 \\
1.0 \\
0.5 \\
<0.5 \\
1.0 \\
1.0 \\
<0.5 \\
0.5\end{array}$ & $\begin{array}{l}<1.0 \\
<1.0 \\
<1.0 \\
<1.0 \\
<1.0 \\
<1.0 \\
<1.0 \\
<1.0\end{array}$ & $\begin{array}{l}<0.5 \\
<0.5 \\
<0.5 \\
<0.5 \\
<0.5 \\
<0.5 \\
<0.5 \\
<0.5\end{array}$ & $\begin{array}{l}<1.0 \\
<1.0 \\
<1.0 \\
<1.0 \\
<1.0 \\
<1.0 \\
<1.0 \\
<1.0\end{array}$ & $\begin{array}{l}<1.0 \\
<1.0 \\
<1.0 \\
<1.0 \\
<1.0 \\
<1.0 \\
<1.0 \\
<1.0\end{array}$ & $\begin{array}{l}<1.0 \\
<1.0 \\
<1.0 \\
<1.0 \\
<1.0 \\
<1.0 \\
<1.0 \\
<1.0\end{array}$ & $\begin{array}{l}<20.0 \\
<20.0 \\
<20.0 \\
<20.0 \\
<20.0 \\
<20.0 \\
<20.0 \\
<20.0\end{array}$ & $\begin{array}{l}<10.0 \\
<10.0 \\
<10.0 \\
<10.0 \\
<10.0 \\
<10.0 \\
<10.0 \\
<10.0\end{array}$ & $\begin{array}{l}<10.0 \\
<10.0 \\
<10.0 \\
<10.0 \\
<10.0 \\
<10.0 \\
<10.0 \\
<10.0\end{array}$ & $\begin{array}{l}2.0 \\
2.0 \\
2.0 \\
2.0 \\
4.0 \\
1.0 \\
1.0 \\
2.0\end{array}$ & $\begin{array}{l}<6.0 \\
<6.0 \\
<6.0 \\
<6.0 \\
<6.0 \\
<6.0 \\
<6.0 \\
<6.0\end{array}$ \\
\hline Mean & Whole $10 \mathrm{~g}$ & 89 & $<0.8$ & $<1.0$ & $<0.5$ & $<1.0$ & $<1.0$ & $<1.0$ & $<20.0$ & $<10.0$ & $<10.0$ & 2.0 & $<6.0$ \\
\hline
\end{tabular}


TABLE B-35 (Continued)

\begin{tabular}{|c|c|c|c|c|c|c|c|c|c|c|c|c|c|}
\hline $\begin{array}{l}\text { [GA Log No. (GLCC } \\
\text { Log No., Lot No.)] } \\
\text { GA Specimen No. }\end{array}$ & $\begin{array}{l}\text { Location } \\
\text { in Log }\end{array}$ & Ash & B & $\mathrm{Fe}$ & V & $\mathrm{Ti}$ & $\mathrm{S}$ & A1 & $\mathrm{Ca}$ & $\mathrm{Si}$ & $\mathrm{Na}$ & $\mathrm{Mg}$ & $\mathrm{Pb}$ \\
\hline \multicolumn{14}{|l|}{$[6484-41(184,426)]$} \\
\hline $6484-41-3 A-L 016$ & MLC & 40 & 1.0 & $<1.0$ & $<0.5$ & $<1.0$ & $<1.0$ & $<1.0$ & $<20.0$ & $<10.0$ & $<10.0$ & $<0.5$ & $<6.0$ \\
\hline$-3 B-L 016$ & MLC & 40 & 4.0 & $<1.0$ & $<0.5$ & $<1.0$ & $<1.0$ & $<1.0$ & $<20.0$ & $<10.0$ & $<10.0$ & $<0.5$ & $<6.0$ \\
\hline$-3 A-L 036$ & MLE & 34 & 1.0 & $<1.0$ & $<0.5$ & $<1.0$ & $<1.0$ & $<1.0$ & $<20.0$ & $<10.0$ & $<10.0$ & $<0.5$ & $<6.0$ \\
\hline$-3 B-L 036$ & MIE & 36 & 2.0 & $<1.0$ & $<0.5$ & $<1.0$ & $<1.0$ & $<1.0$ & $<20.0$ & $<10.0$ & $<10.0$ & $<0.5$ & $<6.0$ \\
\hline$-1 \mathrm{~A}-\mathrm{L} 016$ & $\mathrm{EC}$ & 55 & 0.5 & $<1.0$ & $<0.5$ & $<1.0$ & $<1.0$ & $<1.0$ & $<20.0$ & $<10.0$ & $<10.0$ & $<0.5$ & $<6.0$ \\
\hline$-1 B-L 016$ & $\mathrm{EC}$ & 37 & 1.0 & $<1.0$ & $<0.5$ & $<1.0$ & $<1.0$ & $<1.0$ & $<20.0$ & $<10.0$ & $<10.0$ & $<0.5$ & $<6.0$ \\
\hline$-1 \mathrm{~A}-\mathrm{L} 036$ & $\mathrm{EE}$ & 31 & 1.0 & $<1.0$ & $<0.5$ & $<1.0$ & $<1.0$ & $<1.0$ & $<20.0$ & $<10.0$ & $<10.0$ & $<0.5$ & $<6.0$ \\
\hline$-1 B-L 036$ & $\mathrm{EE}$ & 57 & 1.0 & $<1.0$ & $<0.5$ & $<1.0$ & $<1.0$ & $<1.0$ & $<20.0$ & $<10.0$ & $<10.0$ & $<0.5$ & $<6.0$ \\
\hline Mean & Whole log & 41 & $<1.4$ & $<1.0$ & $<0.5$ & $<1.0$ & $<1.0$ & $<1.0$ & $<20.0$ & $<10.0$ & $<10.0$ & $<0.5$ & $<6.0$ \\
\hline Mean & $\begin{array}{l}\text { Lot } 426 \\
\text { (4 1ogs) }\end{array}$ & $<45$ & $<2.5$ & $<3.2$ & $<0.5$ & $<1.3$ & $<1.5$ & $<4.3$ & $<21.9$ & $<21.3$ & $<10.3$ & $<0.9$ & $<7.1$ \\
\hline Mean & $\begin{array}{l}\text { All logs, } \\
\text { lots } 408 \\
\text { and } 426 \\
\text { (5 logs) }\end{array}$ & $<48$ & $<2.4$ & $<3.0$ & $<0.5$ & $<1.2$ & $<1.6$ & $<3.7$ & $<21.5$ & $<26.0$ & $<10.3$ & $<0.8$ & $<6.9$ \\
\hline
\end{tabular}

(a) Analyses for all elements listed except sulfur were obtained by a standard emission spectrograph technique on 20-mg samples. Other elements detected (1ots 426 and 408 only) but not listed are as follows: $\mathrm{Lu}, \mathrm{Ba}, \mathrm{Be}, \mathrm{Zr}, \mathrm{Ag}<0.5 \mathrm{ppm}$; Li, $\mathrm{Sc}, \mathrm{Mn}, \mathrm{Cu}$, Mo $<1.0 \mathrm{ppm} ; \mathrm{Bi}<2.0 \mathrm{ppm}$; Co, In, Tm, Ni<4.0 ppm; $\mathrm{Nb}, \mathrm{Sn}, \mathrm{Er}$ $<6.0 \mathrm{ppm} ; \mathrm{Sb}<8.0 \mathrm{ppm}$; K, Cr, Cd, La, Eu, Gd, Ha, Yb <10.0 ppm; Zn, Hf, Tl, Dy <20.0 ppm; Rb, Sr, Ta, W, Tb $<40.0 \mathrm{ppm}$; Ce $<80.0 \mathrm{ppm} ; \mathrm{Sm}$, Pr, Cs, P <100.0 ppm; Nd $<200.0 \mathrm{ppm}$. The symbol< means less than the sensitivity of the spectrographic procedure used. Results for individual analysis are correct within a factor of $40 \%$ or one standard deviation.

(b) Not measured.

(c) Insufficient material for proper analysis. Values not used in mean value calculation. 
TABLE B-36

IMPURITY CONTENT OF TS-1240, LOT 1

[in ppm(a)]

\begin{tabular}{|c|c|c|c|c|c|c|c|c|c|c|c|c|c|c|c|}
\hline $\begin{array}{l}\text { [GA Log No. } \\
\text { (UCC Log No.)] } \\
\text { GA Specimen No. }\end{array}$ & $\begin{array}{l}\text { Location } \\
\text { in Log }\end{array}$ & Ash & B & $\mathrm{Fe}$ & V & $\mathrm{Ti}$ & $S$ & Al & $\mathrm{Ca}$ & $\mathrm{Si}$ & $\mathrm{Na}$ & $\mathrm{Mg}$ & $\mathrm{Pb}$ & $\mathrm{Ba}$ & $\mathrm{Ni}$ \\
\hline \multicolumn{16}{|l|}{$\left[\begin{array}{lll}5651-72 & (30)\end{array}\right]$} \\
\hline 5651-72-3A-L082 & QLC & 94 & 1.0 & $<10.0$ & 20.0 & 20.0 & 5.0 & & & & & & & & \\
\hline -3B-L094 & QLC & 120 & 1.0 & $<10.0$ & 10.0 & 20.0 & 5.0 & $<1.0$ & $<20.0$ & $<10.0$ & $<10.0$ & $<0.5$ & $<6.0$ & $<0.5$ & $<4.0$ \\
\hline$-3 A-L 156$ & QLE & 140 & 1.0 & $<10.0$ & 10.0 & 10.0 & 10.0 & & & & & & & & \\
\hline$-3 \mathrm{~B}-\mathrm{L} 165$ & QLE & 92 & 1.0 & $<10.0$ & 10.0 & 10.0 & 13.0 & & & & & & & & \\
\hline$-1 \mathrm{~A}-\mathrm{L} 031$ & $\mathrm{EC}$ & 68 & 1.0 & $<10.0$ & 20.0 & 20.0 & 22.0 & & & & & & & & \\
\hline$-1 \mathrm{~B}-\mathrm{L} 043$ & $\mathrm{EC}$ & 69 & 1.0 & $<10.0$ & 10.0 & 10.0 & 18.0 & $<1.0$ & $<20.0$ & $<10.0$ & $<10.0$ & $<0.5$ & $<6.0$ & $<0.5$ & $<4.0$ \\
\hline$-1 \mathrm{~A}-\mathrm{L} 106$ & $\mathrm{EE}$ & 110 & $<0.5$ & $<10.0$ & 10.0 & 10.0 & $<7.0$ & & & & & & & & \\
\hline$-1 \mathrm{~B}-\mathrm{L} 115$ & $\mathrm{EE}$ & 120 & $<0.5$ & $<10.0$ & 10.0 & 10.0 & 25.0 & & & & & & & & \\
\hline Mean & Whole $\log$ & 89 & $<0.9$ & $<10.0$ & 12.5 & 13.7 & $<13.0$ & $<1.0$ & $<20.0$ & $<10.0$ & $<10.0$ & $<0.5$ & $<6.0$ & $<0.5$ & $<4.0$ \\
\hline \multicolumn{16}{|l|}{$\left[\begin{array}{lll}5651-73 & (40)\end{array}\right]$} \\
\hline 5651-73-3A-L005 & MLC & $<98$ & $<0.5$ & $<1.0$ & 10.0 & 10.0 & $<2.0$ & $<1.0$ & $<20.0$ & $<10.0$ & $<10.0$ & $<0.5$ & $<6.0$ & $<0.5$ & $<4.0$ \\
\hline$-\mathrm{L} 007$ & MLC & $<39$ & $<0.5$ & 4.0 & 10.0 & 10.0 & $<2.0$ & 40.0 & $<20.0$ & $<10.0$ & $<10.0$ & 2.0 & $<6.0$ & 6.0 & $<4.0$ \\
\hline$-\mathrm{L} 009$ & MLE & $<30$ & $<0.5$ & $<1.0$ & 20.0 & 10.0 & 22.0 & $<1.0$ & $<20.0$ & $<10.0$ & $<10.0$ & $<0.5$ & $<6.0$ & 0.5 & $<4.0$ \\
\hline$-\mathrm{L} 010$ & MLE & $<31$ & $<0.5$ & $<1.0$ & 10.0 & 8.0 & 10.0 & $<1.0$ & $<20.0$ & $<10.0$ & $<10.0$ & $<0.5$ & $<6.0$ & 4.0 & $<4.0$ \\
\hline Mean & ML position & $<42$ & $<0.5$ & $<1.7$ & 12.5 & 9.5 & $<9.0$ & $<10.8$ & $<20.0$ & $<10.0$ & $<10.0$ & $<0.9$ & $<6.0$ & $<2.8$ & $<4.0$ \\
\hline \multicolumn{16}{|l|}{$\left[\begin{array}{lll}5651-75 & (46)\end{array}\right]$} \\
\hline $5651-75-3 A-L 082$ & MLC & 95 & $<0.5$ & $<10.0$ & 10.0 & 20.0 & 5.0 & & & & & & & & \\
\hline$-3 \mathrm{~B}-\mathrm{L} 094$ & MLC & 81 & $<0.5$ & $<10.0$ & 10.0 & 20.0 & 18.0 & $<1.0$ & $<20.0$ & $<10.0$ & $<10.0$ & $<0.5$ & $<6.0$ & $<0.5$ & $<4.0$ \\
\hline$-3 A-L 156$ & MLE & 74 & $<0.5$ & $<10.0$ & 20.0 & 20.0 & 15.0 & & & & & & & & \\
\hline$-3 B-L 165$ & MLE & 47 & $<0.5$ & $<10.0$ & 10.0 & 20.0 & 13.0 & & & & & & & & \\
\hline$-1 \mathrm{~A}-\mathrm{L} 031$ & $\mathrm{EC}$ & 55 & $<0.5$ & $<10.0$ & 10.0 & 20.0 & 9.0 & & & & & & & & \\
\hline$-1 \mathrm{~B}-\mathrm{L} 043$ & EC & 89 & $<0.5$ & $<10.0$ & 20.0 & 20.0 & 18.0 & $<1.0$ & $<20.0$ & $<10.0$ & $<10.0$ & $<0.5$ & $<6.0$ & $<0.5$ & $<4.0$ \\
\hline$-1 \mathrm{~A}-\mathrm{L} 106$ & $\mathrm{EE}$ & 58 & $<0.5$ & $<10.0$ & 10.0 & 10.0 & 17.0 & & & & & & & & \\
\hline$-1 B-L 115$ & $\mathrm{EE}$ & 39 & $<0.5$ & $<10.0$ & 20.0 & 20.0 & 18.0 & & & & & & & & \\
\hline Mean & Whole log & 67 & $<0.5$ & $<10.0$ & 13.7 & 18.7 & 14.2 & $<1.0$ & $<20.0$ & $<10.0$ & $<10.0$ & $<0.5$ & $<6.0$ & $<0.5$ & $<4.0$ \\
\hline
\end{tabular}


TABLE B-36 (Continued)

\begin{tabular}{|c|c|c|c|c|c|c|c|c|c|c|c|c|c|c|c|}
\hline $\begin{array}{l}\text { [GA Log No. } \\
\text { (UCC Log No.)] } \\
\text { GA Specimen No. }\end{array}$ & $\begin{array}{l}\text { Location } \\
\text { in Log }\end{array}$ & Ash & B & $\mathrm{Fe}$ & V & $\mathrm{Ti}$ & $\mathrm{S}$ & $\mathrm{A} 1$ & $\mathrm{Ca}$ & $\mathrm{Si}$ & $\mathrm{Na}$ & $\mathrm{Mg}$ & $\mathrm{Pb}$ & $\mathrm{Ba}$ & $\mathrm{Ni}$ \\
\hline$[5651-74(42)]$ & & & & & & & & & & & & & & & \\
\hline $\begin{array}{r}5651-74-3 \mathrm{~A}-\mathrm{L} 082 \\
-3 \mathrm{~B}-\mathrm{L} 094 \\
-3 \mathrm{~A}-\mathrm{L} 156 \\
-3 \mathrm{~B}-\mathrm{L} 165 \\
-1 \mathrm{~A}-\mathrm{L} 031 \\
-1 \mathrm{~B}-\mathrm{L} 043 \\
-1 \mathrm{~A}-\mathrm{L} 106 \\
-1 \mathrm{~B}-\mathrm{L} 115\end{array}$ & $\begin{array}{l}\text { MLC } \\
\text { MLC } \\
\text { MLE } \\
\text { MLE } \\
\text { EC } \\
\text { EC } \\
\text { EE } \\
\text { EE }\end{array}$ & $\begin{array}{r}<44 \\
<28 \\
<35 \\
<27 \\
<50 \\
<40 \\
53 \\
<35\end{array}$ & $\begin{aligned}<1.0 \\
<1.0 \\
<1.0 \\
\\
2.0 \\
<1.0 \\
<1.0 \\
<1.0 \\
<1.0\end{aligned}$ & $\begin{array}{l}<10.0 \\
<10.0 \\
<10.0 \\
<10.0 \\
<10.0 \\
<10.0 \\
<10.0 \\
<10.0\end{array}$ & $\begin{array}{r}<10.0 \\
60.0 \\
40.0 \\
<10.0 \\
<10.0 \\
40.0 \\
<10.0 \\
40.0\end{array}$ & $\begin{array}{r}<10.0 \\
40.0 \\
20.0 \\
<10.0 \\
<10.0 \\
40.0 \\
<10.0 \\
40.0\end{array}$ & $\begin{array}{r}<1.5 \\
<1.5 \\
5.5 \\
3.5 \\
3.5 \\
2.5 \\
3.5 \\
5.0\end{array}$ & $\begin{array}{l}<1.0 \\
<1.0 \\
<1.0 \\
<1.0 \\
<1.0 \\
<1.0 \\
<1.0 \\
10.0\end{array}$ & $\begin{array}{l}<20.0 \\
<20.0 \\
<20.0 \\
<20.0 \\
<20.0 \\
<20.0 \\
<20.0 \\
<20.0\end{array}$ & $\begin{array}{r}<10.0 \\
<10.0 \\
10.0 \\
<10.0 \\
<10.0 \\
<10.0 \\
<10.0 \\
<10.0\end{array}$ & & $\begin{array}{r}<0.5 \\
<0.5 \\
6.0 \\
4.0 \\
2.0 \\
0.5 \\
1.0 \\
<0.5\end{array}$ & $\begin{array}{l}<6.0 \\
<6.0 \\
<6.0 \\
<6.0 \\
<6.0 \\
<6.0 \\
<6.0 \\
<6.0\end{array}$ & $\begin{array}{l}<0.5 \\
<0.5 \\
<0.5 \\
<0.5 \\
<0.5 \\
<0.5 \\
<0.5 \\
<0.5\end{array}$ & $\begin{array}{l}<4.0 \\
<4.0 \\
<4.0 \\
<4.0 \\
<4.0 \\
<4.0 \\
<4.0 \\
<4.0\end{array}$ \\
\hline $\begin{array}{l}\text { Mean } \\
{[6484-29} \\
[8-1 S-I D-23)]\end{array}$ & Whole $\log$ & $<39$ & $<1.1$ & $<10.0$ & $<27.5$ & $<22.5$ & $<3.2$ & $<2.1$ & $<20.0$ & $<10.0$ & -- & $<1.9$ & $<6.0$ & $<0.5$ & $<4.0$ \\
\hline $\begin{array}{r}6484-29-3 \mathrm{~A}-\mathrm{L} 082 \\
-3 \mathrm{~B}-\mathrm{L} 094 \\
-3 \mathrm{~A}-\mathrm{L} 156 \\
-3 \mathrm{~B}-\mathrm{L} 165 \\
-1 \mathrm{~A}-\mathrm{L} 031 \\
-1 \mathrm{~B}-\mathrm{L} 043 \\
-1 \mathrm{~A}-\mathrm{L} 106 \\
-1 \mathrm{~B}-\mathrm{L} 115\end{array}$ & $\begin{array}{l}\text { MLC } \\
\text { MLC } \\
\text { MLE } \\
\text { MLE } \\
\text { EC } \\
\text { EC } \\
\text { EE } \\
\text { EE }\end{array}$ & $\begin{array}{r}250 \\
204 \\
138 \\
66 \\
125 \\
171 \\
102 \\
257\end{array}$ & $\begin{array}{r}0.5 \\
<0.5 \\
<0.5 \\
<0.5 \\
<0.5 \\
<0.5 \\
<0.5 \\
<0.5\end{array}$ & $\begin{array}{l}40.0 \\
40.0 \\
10.0 \\
40.0 \\
20.0 \\
10.0 \\
20.0 \\
10.0\end{array}$ & $\begin{array}{r}40.0 \\
40.0 \\
10.0 \\
40.0 \\
20.0 \\
8.0 \\
40.0 \\
20.0\end{array}$ & $\begin{array}{r}20.0 \\
20.0 \\
20.0 \\
20.0 \\
20.0 \\
8.0 \\
20.0 \\
10.0\end{array}$ & $\begin{array}{r}2.0 \\
4.0 \\
12.0 \\
8.5 \\
12.0 \\
8.0 \\
9.5 \\
5.0\end{array}$ & $\begin{array}{l}20.0 \\
<1.0 \\
<1.0 \\
<1.0 \\
<1.0 \\
<1.0 \\
10.0 \\
10.0\end{array}$ & $\begin{array}{l}60.0 \\
40.0 \\
20.0 \\
20.0 \\
20.0 \\
20.0 \\
40.0 \\
40.0\end{array}$ & $\begin{array}{l}-- \\
20.0 \\
20.0 \\
40.0 \\
40.0 \\
40.0 \\
60.0 \\
20.0\end{array}$ & $\begin{array}{l}<10.0 \\
20.0 \\
-- \\
10.0 \\
10.0 \\
<10.0 \\
<10.0 \\
<10.0\end{array}$ & $\begin{array}{r}2.0 \\
<0.5 \\
<0.5 \\
<0.5 \\
<0.5 \\
<0.5 \\
2.0 \\
2.0\end{array}$ & $\begin{array}{l}<6.0 \\
<6.0 \\
<6.0 \\
<6.0 \\
<6.0 \\
<6.0 \\
<6.0 \\
<6.0\end{array}$ & $\begin{array}{l}10.0 \\
10.0 \\
10.0 \\
10.0 \\
10.0 \\
10.0 \\
10.0 \\
10.0\end{array}$ & $\begin{array}{l}8.0 \\
8.0 \\
6.0 \\
8.0 \\
8.0 \\
4.0 \\
8.0 \\
6.0\end{array}$ \\
\hline Mean & Whole log & 157 & $<0.5$ & 23.7 & 28.5 & 15.7 & 7.6 & $<5.6$ & 32.5 & 34.3 & $<11.4$ & $<1.1$ & $<6.0$ & 10.0 & 7.0 \\
\hline Mean & $\begin{array}{l}\text { Whole } \\
\text { lot } 1 \\
\text { (5 logs) }\end{array}$ & $<78.8$ & $<0.7$ & $<11.1$ & $<18.9$ & $<16.0$ & $<9.4$ & $<4.1$ & $<22.5$ & $<14.9$ & $<10.4$ & $<1.0$ & $<6.0$ & $<2.9$ & $<4.6$ \\
\hline
\end{tabular}

(a) Analyses for all elements 1isted except sulfur were obtained by a standard emission spectrograph technique on 20-mg samples. Other elements detected but not Iisted are as follows: $\mathrm{Be}, \mathrm{Zr}, \mathrm{Ag}<0.5 \mathrm{ppm} ; \mathrm{Li}, \mathrm{Sc}, \mathrm{Mn}, \mathrm{Cu}, \mathrm{Mo}<1.0$ $\mathrm{ppm} ; \mathrm{Bi}<2.0 \mathrm{ppm}$; $\mathrm{Co}$, In, $\mathrm{Tm}<4.0 \mathrm{ppm} ; \mathrm{Nb}, \mathrm{Sn}, \mathrm{Er}<6.0 \mathrm{ppm} ; \mathrm{Sb}<8.0 \mathrm{ppm} ; \mathrm{K}, \mathrm{Cr}, \mathrm{Cd}, \mathrm{La}, \mathrm{Eu}, \mathrm{Gd}, \mathrm{Ho}, \mathrm{Yb}<10.0 \mathrm{ppm}$; $\mathrm{Zn}$, Hf, T1, Dy $<20.0 \mathrm{ppm}$; Rb, Sr, Ta, W, Tb $<40.0 \mathrm{ppm} ; \mathrm{Ce}<80.0 \mathrm{ppm} ; \mathrm{Sm}, \mathrm{Pr}, \mathrm{Cs}, \mathrm{P}<100.0 \mathrm{ppm} ; \mathrm{Nd}<200.0 \mathrm{ppm}$. The symbol < means less than the sensitivity of the spectrographic procedure used. Results for individual analyses are correct within a factor of $40 \%$ or one standard deviation. 
TABLE $\quad B-37$

BURNABLE AND NONBURNABLE BORON EQUIVALENT VALUES FOR H-451 AND TS-1240

\begin{tabular}{|c|c|c|c|c|c|c|}
\hline \multirow[b]{2}{*}{ Element } & \multirow{2}{*}{$\begin{array}{c}\text { Boron } \\
\text { Equivalent }\end{array}$} & \multicolumn{2}{|c|}{$\begin{array}{l}\text { Impurity Content, } \\
\text { Less Than Value Given } \\
(\mathrm{ppm})\end{array}$} & \multicolumn{2}{|c|}{$\begin{array}{c}\text { Upper Limit of Boron } \\
\text { Equivalent Value } \\
\text { (ppm) }\end{array}$} & \multirow{2}{*}{$\begin{array}{l}\text { Analytical } \\
\text { Methods (a) }\end{array}$} \\
\hline & & $\mathrm{H}-451$ & TS -1230 & $\mathrm{H}-451$ & $\mathrm{TS}-1240$ & \\
\hline
\end{tabular}

\begin{tabular}{|c|c|c|c|c|c|c|}
\hline & & & NBURNABLE & & & \\
\hline $\mathrm{Ag}$ & 0.0322 & 0.5 & 0.5 & 0.0161 & 0.0161 & 1 \\
\hline AI & 0.00012 & 3.7 & 4.1 & 0.0004 & 0.0005 & 1 \\
\hline $\mathrm{Ba}$ & 0.00042 & 0.5 & 2.9 & 0.0002 & 0.0012 & 1 \\
\hline $\mathrm{Ca}$ & 0.00020 & 21.5 & 22.5 & 0.0043 & 0.0045 & 1 \\
\hline $\mathrm{Cl}$ & 0.0136 & $3.0^{(b)}$ & 1.0 & $0.0408^{(b)}$ & 0.0136 & 2 \\
\hline Co & 0.0114 & 4.0 & 4.0 & 0.0456 & 0.0456 & 1 \\
\hline $\mathrm{Cr}$ & 0.00086 & 1.0 & 1.0 & 0.0009 & 0.0009 & 2 \\
\hline Cs & 0.0127 & 1.0 & 1.0 & 0.0127 & 0.0127 & 2 \\
\hline $\mathrm{Cu}$ & 0.00099 & $0.07^{(b)}$ & $0.47^{\text {(b) }}$ & $0.0001^{(b)}$ & $0.0004^{(b)}$ & 3 \\
\hline Dy & 0.1048 & 0.12 & 0.16 & 0.0126 & 0.0168 & 3 \\
\hline Er & 0.0692 & 0.03 & 0.04 & 0.0021 & 0.0028 & 3 \\
\hline $\mathrm{Fe}$ & 0.00068 & 3.0 & 11.1 & 0.0020 & 0.0076 & 1 \\
\hline Hf & 0.0475 & 0.17 & 0.16 & 0.0081 & 0.0076 & 3 \\
\hline Ho & 0.0222 & 0.08 & 0.08 & 0.0018 & 0.0018 & 3 \\
\hline In & 0.135 & 1.0 & 1.0 & 0.5400 & 0.5400 & 2 \\
\hline $\mathrm{K}$ & 0.00081 & 1.0 & 1.0 & 0.0008 & 0.0008 & 2 \\
\hline $\mathrm{La}$ & 0.00114 & 0.03 & 1.38 & 0.0000 & 0.0016 & 3 \\
\hline $\mathrm{Sn}$ & 0.00029 & 1.0 & 1.0 & 0.0003 & 0.0003 & 2 \\
\hline $\mathrm{Lu}$ & 0.048 & 0.02 & 0.01 & 0.0010 & 0.0010 & 3 \\
\hline $\mathrm{Mg}$ & 0.00005 & 0.8 & 1.0 & 0.0000 & 0.0001 & 1 \\
\hline Mo & 0.0014 & 1.0 & 1.0 & 0.0014 & 0.0014 & 1 \\
\hline Mn & 0.0041 & 1.0 & 1.0 & 0.0041 & 0.0041 & 1 \\
\hline $\mathrm{Na}$ & 0.00034 & 10.3 & 10.4 & 0.0035 & 0.0035 & 1 \\
\hline $\mathrm{Nd}$ & 0.00573 & 0.3 & 0.36 & 0.0017 & 0.0021 & 3 \\
\hline $\mathrm{Ni}$ & 0.00114 & 4.0 & 4.6 & 0.0046 & 0.0052 & 1 \\
\hline $\operatorname{Pr}$ & 0.00156 & 0.42 & 0.64 & 0.0007 & 0.0010 & 3 \\
\hline S & 0.00029 & 1.6 & 9.4 & 0.0005 & 0.0027 & 4 \\
\hline $\mathrm{Sb}$ & 0.0062 & 1.0 & 1.0 & 0.0062 & 0.0062 & 2 \\
\hline $\mathrm{Sc}$ & 0.0074 & 0.01 & 0.01 & 0.0007 & 0.0007 & 3 \\
\hline Si & 0.000085 & 26.0 & 14.9 & 0.0022 & 0.0013 & 1 \\
\hline $\mathrm{Ta}$ & 0.0159 & 0.35 & 0.32 & 0.0056 & 0.0051 & 3 \\
\hline $\mathrm{Tb}$ & 0.0137 & 0.20 & 0.26 & 0.0027 & 0.0036 & 3 \\
\hline $\mathrm{T} \mathbf{i}$ & 0.00180 & 1.2 & 16.0 & 0.0022 & 0.0286 & 1 \\
\hline $\operatorname{Tm}$ & 0.0487 & 0.04 & 0.03 & 0.0019 & 0.0015 & 3 \\
\hline V & 0.00147 & 0.5 & 18.9 & 0.0007 & 0.0278 & 1 \\
\hline W & 0.00857 & $25.5^{(b)}$ & 1.0 & $0.2185(\mathrm{~b})$ & 0.0086 & 1 \\
\hline $\mathrm{Yb}$ & 0.00715 & 0.03 & 0.06 & 0.0002 & 0.0004 & 3 \\
\hline $\mathrm{P}$ & 0.00010 & 1.0 & 1.0 & 0.0001 & 0.0001 & 2 \\
\hline $\mathrm{Pb}$ & 0.000001 & 6.9 & 6.0 & 0.0000 & 0.0000 & 1 \\
\hline $\mathrm{Rb}$ & 0.00033 & 1.0 & 1.0 & 0.0003 & 0.0003 & 2 \\
\hline $\mathrm{T} 1$ & 0.00043 & 1.0 & 1.0 & 0.0004 & 0.0004 & 2 \\
\hline $\mathrm{Zn}$ & 0.00027 & 1.0 & 1.0 & 0.0003 & 0.0003 & 2 \\
\hline $\mathrm{Zr}$ & 0.00010 & 0.08 & 0.5 & 0.0000 & 0.0001 & 2 \\
\hline $\mathrm{Bi}$ & 0.000026 & 1.0 & 1.0 & 0.0000 & 0.0000 & 2 \\
\hline $\mathrm{Nb}$ & 0.00088 & 0.05 & 1.74 & 0.0000 & 0.0015 & 3 \\
\hline $\mathrm{N}$ & 0.002 & -- & -- & -- & -- & -- \\
\hline 0 & 0.000004 & -- & -- & -- & -- & - \\
\hline $\mathrm{Sr}$ & 0.000836 & 0.30 & 0.47 & 0.0003 & 0.0004 & 3 \\
\hline $\mathrm{Ce}$ & 0.0001 & 0.30 & 0.56 & 0.0000 & 0.0001 & 3 \\
\hline $\mathrm{Be}$ & 0.000016 & 0.005 & 0.00 & 0.0000 & 0.0000 & 3 \\
\hline & . & lue, (c) & & 0.9486 & 0.7839 & \\
\hline
\end{tabular}


TABLE B-37 (Continued)

\begin{tabular}{|c|c|c|c|c|c|c|}
\hline \multirow[b]{2}{*}{ Element } & \multirow{2}{*}{$\begin{array}{l}\text { Boron } \\
\text { Equivalent }\end{array}$} & \multicolumn{2}{|c|}{$\begin{array}{c}\text { Impurity Content, } \\
\text { Less Than Value Given } \\
\text { (ppm) }\end{array}$} & \multicolumn{2}{|c|}{$\begin{array}{c}\text { Upper Limit of Boron } \\
\text { Equivalent Value } \\
\text { (ppm) }\end{array}$} & \multirow{2}{*}{$\begin{array}{l}\text { Analytical } \\
\text { Methods }(a)\end{array}$} \\
\hline & & $\mathrm{H}-451$ & $\mathrm{TS}-1230$ & $\mathrm{H}-451$ & TS -1240 & \\
\hline \multicolumn{7}{|c|}{ BURNABLE } \\
\hline B & 1.0 & 2.4 & 0.7 & 2.4 & 0.7 & 1 \\
\hline $\mathrm{Cd}$ & 0.855 & 0.5 & 0.5 & 0.4275 & 0.4275 & 5 \\
\hline Eu & 0.459 & 0.04 & 0.06 & 0.0183 & 0.0275 & 3 \\
\hline $\mathrm{Gd}$ & 1.57 & 0.05 & 0.08 & 0.0785 & 0.1256 & 3 \\
\hline Sin & 0.941 & 0.39 & 0.61 & 0.3670 & 0.5740 & 3 \\
\hline $\mathrm{Li}$ & 0.171 & 0.005 & 0.005 & 0.0009 & 0.0009 & 6 \\
\hline \multicolumn{4}{|c|}{$\begin{array}{l}\text { Burnable boron equivalent value, } \\
\text { less than value given (ppm) }\end{array}$} & 3.2922 & 1.8560 & \\
\hline
\end{tabular}

(a) Method 1. Standard emission spectroscopy on 20-mg samples (Section A.5.2). Values represent means of approximately 40 measurements. Results are correct within a factor of approximately $40 \%$ or one standard deviation.

Method 2. Spark mass spectroscopy (Section A.5.3) (work performed by Accu-Labs Research, Inc., Wheat Ridge, Colorado). Value represents mean of two measurements. Results are correct within approximately 200 to $300 \%$.

Method 3. Same as 1 above except samples were preconcentrated to 20 to $50 \mathrm{mg}$. Samples of 10 to $20 \mathrm{~g}$ were preconcentrated by ashing in still air at $750^{\circ} \mathrm{C}$. Value represents mean of two measurements. Results are correct within $40 \%$ or one standard deviation.

Method 4. Wet chemical analysis (Section A.5.4). Mean of approximately 40 measurements.

Method 5. Same as 1 above but on a single 50-mg sample. Results are correct within approximately $40 \%$ or one standard deviation.

Method 6. Isotopic dilution spark mass spectroscopy (Section A.5.5). Work done by Oak Ridge National Laboratory (ORNL) on $10-g$ samples.

(b) Actual values.

(c) Does not include boron equivalent contributions from 0 and $N$. 
TABLE B-38

IMPURITY CONTENT OF H-451 AND TS-1240

[in $\mathrm{ppm}(\mathrm{a})]$

\begin{tabular}{|c|c|c|c|c|c|c|c|c|c|}
\hline \multirow[b]{2}{*}{ E1ement } & \multicolumn{3}{|c|}{$\begin{array}{c}\text { H-451 } \\
\text { GA Log No. } \\
\text { (GLCC Log No./GLCC Lot No.) }\end{array}$} & \multicolumn{5}{|c|}{$\begin{array}{c}\text { TS }-1240 \\
\text { GA Log No. } \\
\text { (UCC Log No./UCC Lot No.) }\end{array}$} & \multirow[b]{2}{*}{$\operatorname{Mean}(\mathrm{d})$} \\
\hline & $\begin{array}{c}5651-86 \\
(52 / 408)(b)\end{array}$ & $\begin{array}{c}6484-34 \\
(198 / 426)(c)\end{array}$ & $\operatorname{Mean}^{(\mathrm{d})}$ & $\begin{array}{l}5651-72 \\
(30 / 1)(b)\end{array}$ & $\begin{array}{l}5651-73 \\
(40 / 1)^{(b)}\end{array}$ & $\begin{array}{c}5651-74 \\
(42 / 1)(b)\end{array}$ & $\begin{array}{l}5651-75 \\
(46 / 1)(b)\end{array}$ & $\begin{array}{c}6484-29 \\
(8-1 \mathrm{~S}-\mathrm{ID}-23 / 1)\end{array}$ & \\
\hline $\mathrm{Ag}^{(\mathrm{f})}$ & 0.06 & & $\rightarrow$ & 0.01 & 0.30 & 0.30 & 0.22 & & -- \\
\hline $\mathrm{Ba}$ & 7.50 & & 7.50 & 5.25 & 3.00 & 4.50 & 1.88 & & 3.66 \\
\hline $\mathrm{Ca}$ & 6.00 & & 6.00 & $>7.50$ & 7.50 & $>7.50$ & 7.50 & & 37.50 \\
\hline $\mathrm{Co}^{(\mathrm{g})}$ & $<0.03$ & & $<0.03$ & $<0.03$ & 3.00 & 0.30 & 3.75 & & $<1.77$ \\
\hline $\mathrm{Cu}$ & 0.07 & & 0.07 & 0.06 & 0.75 & 0.30 & 0.75 & & 0.47 \\
\hline $\mathrm{Eu}$ & $<0.07$ & $<0.02$ & $<0.04$ & $<0.07$ & $<0.07$ & $<0.07$ & $<0.07$ & $<0.02$ & $<0.06$ \\
\hline $\mathrm{HF}$ & $<0.15$ & $<0.20$ & $<0.17$ & $<0.15$ & $<0.15$ & $<0.15$ & $<0.15$ & $<0.20$ & $<0.16$ \\
\hline $\mathrm{K}^{(\mathrm{g})}$ & 3.00 & & 3.00 & 1.50 & 1.50 & 0.45 & 3.00 & & 1.61 \\
\hline $\mathrm{Lu}_{(\mathrm{g})}$ & $<0.00$ & $<0.04$ & $<0.02$ & $<0.00$ & $<0.00$ & $<0.00$ & $<0.00$ & $<0.04$ & $<0.01$ \\
\hline Mo $(g)$ & $<0.01$ & & $<0.01$ & 0.15 & 0.75 & 0.45 & 0.15 & & 0.38 \\
\hline$N \mathbf{i}^{(g)}$ & 3.00 & & 3.00 & 4.50 & 4.50 & 7.50 & 3.75 & & 5.06 \\
\hline Pr & $<0.75$ & $<0.20$ & $<0.42$ & $<0.75$ & $<0.75$ & $<0.75$ & $<0.75$ & $<0.20$ & $<0.64$ \\
\hline $\mathrm{Sc}_{(\mathrm{g})}$ & $<0.01$ & & $<0.01$ & $<0.01$ & $<0.01$ & $<0.01$ & $<0.01$ & & $<0.01$ \\
\hline $\mathrm{sn}^{(\mathrm{g})}$ & $<0.04$ & & $<0.04$ & $<0.04$ & 0.60 & $<0.04$ & $<0.04$ & & 0.18 \\
\hline $\mathrm{Tb}$ & $<0.30$ & $<0.10$ & $<0.20$ & $<0.30$ & $<0.30$ & $<0.30$ & $<0.30$ & $<0.10$ & $<0.26$ \\
\hline $\mathrm{Tm}$ & $<0.03$ & $<0.04$ & $<0.04$ & $<0.03$ & $<0.03$ & $<0.03$ & $<0.03$ & $<0.04$ & $<0.03$ \\
\hline $\mathrm{Yb}$ & $<0.07$ & $<0.00$ & $<0.03$ & $<0.07$ & $<0.07$ & $<0.07$ & $<0.07$ & $<0.00$ & $<0.06$ \\
\hline A1 & 1.50 & & 1.50 & 1.50 & 7.50 & 3.00 & 6.00 & & 4.50 \\
\hline $\mathrm{Be}_{(f)}$ & $<0.00$ & & $<0.00$ & $<0.00$ & 0.02 & $<0.00$ & $<0.00$ & & $<0.00$ \\
\hline $\mathrm{Cd}(\mathrm{f})$ & 0.30 & $<0.50^{(h)}$ & $<0.50$ & 0.45 & 0.52 & 0.60 & 0.38 & $<0.50^{(h)}$ & $<0.50$ \\
\hline $\mathrm{Cr}^{(\mathrm{g})}$ & $<0.07$ & & $<0.07$ & 7.50 & 6.75 & 7.50 & $<0.07$ & & $<5.46$ \\
\hline Dy $(g)$ & $<0.15$ & $<0.10$ & $<0.12$ & $<0.15$ & $<0.15$ & $<0.15$ & $<0.15$ & 0.20 & $<0.16$ \\
\hline $\mathrm{Fe}(\mathrm{g})$ & 7.50 & & 7.50 & 7.50 & $>7.50$ & 7.50 & 7.50 & & $>7.50$ \\
\hline Ho & $<0.07$ & $<0.10$ & $<0.08$ & $<0.07$ & $<0.07$ & $<0.07$ & $<0.07$ & $<0.10$ & $<0.08$ \\
\hline La & 0.07 & $<0.00$ & $<0.03$ & 0.07 & 3.00 & 0.07 & 3.75 & $<0.00$ & $<1.38$ \\
\hline $\mathrm{Mg}_{(\alpha)}$ & 0.30 & & 0.30 & 0.60 & 0.30 & 0.75 & 0.45 & & 0.53 \\
\hline $\mathrm{Na}(\mathrm{g})$ & 3.00 & & 3.00 & 4.50 & 3.00 & 1.50 & 4.50 & & 3.38 \\
\hline$P(g)$ & $<0.75$ & & $<0.75$ & $<0.75$ & $<0.75$ & $<0.75$ & $<0.75$ & & $<0.75$ \\
\hline $\mathrm{Rb}(\mathrm{g})$ & $<0.30$ & & $<0.30$ & $<0.30$ & $<0.30$ & $<0.30$ & $<0.30$ & & $<0.30$ \\
\hline $\mathrm{Si}(\mathrm{g})$ & 7.50 & & 7.50 & 1.50 & 4.50 & 3.00 & 3.75 & & 3.19 \\
\hline $\mathrm{Sr}$ & $<0.30$ & & $<0.30$ & $<0.30$ & 0.52 & $<0.30$ & 0.75 & & $<0.47$ \\
\hline $\mathrm{Ti}$ & 3.00 & & 3.00 & $>7.50$ & $>7.50$ & $>7.50$ & $>7.50$ & & $>7.50$ \\
\hline$v(f)$ & 0.75 & & - & $7: 50$ & $>7.50$ & $>7.50$ & $>7.50$ & & -- \\
\hline $\ln (f)$ & 0.15 & & -- & $<0.15$ & $<0.15$ & $<0.15$ & $<0.15$ & & -- \\
\hline$B(f)$ & 0.60 & & -- & 0.60 & 0.30 & 0.07 & $<0.00$ & & -- \\
\hline $\mathrm{Bi}(\mathrm{f})$ & $<0.02$ & & -- & $<0.02$ & $<0.02$ & $<0.02$ & $<0.02$ & & - \\
\hline $\mathrm{Ce}$ & $<0.60$ & $<0.10$ & $<0.30$ & $<0.60$ & $<0.60$ & $<0.60$ & $<0.60$ & 0.40 & $<0.56$ \\
\hline $\mathrm{Cs}^{(\mathrm{D})}$ & $<0.75$ & $<10.00(h)$ & $<10.00$ & $<0.75$ & $<0.75$ & $<0.75$ & $<0.75$ & $<10.00^{(h)}$ & $<10.00$ \\
\hline Er & $<0.04$ & $<0.02$ & $<0.03$ & $<0.04$ & $<0.04$ & $<0.04$ & $<0.04$ & $<0.02$ & $<0.04$ \\
\hline $\mathrm{Gd}$ & $<0.07$ & $<0.04$ & $<0.05$ & $<0.07$ & $<0.07$ & $<0.07$ & $<0.07$ & 0.10 & $<0.08$ \\
\hline $\operatorname{In}(g)$ & $<0.03$ & & $<0.03$ & $<0.03$ & $<0.03$ & $<0.03$ & $<0.03$ & & $<0.03$ \\
\hline$L i(f)$ & 0.07 & & -- & 0.07 & 0.45 & 0.04 & 0.45 & & -- \\
\hline $\mathrm{Mn}(g)$ & 0.15 & & 0.15 & 0.60 & 0.52 & 0.75 & 0.10 & & 0.49 \\
\hline $\mathrm{Nb}$ & $<0.04$ & $<0.06$ & $<0.05$ & 0.45 & 0.60 & 7.50 & 0.07 & $<0.06$ & $<1.74$ \\
\hline $\mathrm{Pb}(\mathrm{g})$ & 0.15 & & 0.15 & $<0.04$ & 0.07 & $<0.04$ & 0.10 & & $<0.06$ \\
\hline$S b(g)$ & $<0.06$ & & $<0.06$ & $<0.06$ & $<0.06$ & $<0.06$ & $<0.06$ & & $<0.06$ \\
\hline $\mathrm{Sm}$ & $<0.75$ & $<0.04$ & $<0.39$ & $<0.75$ & $<0.75$ & $<0.75$ & $<0.75$ & $<0.04$ & $<0.61$ \\
\hline $\mathrm{Ta}$ & $<0.30$ & $<0.40$ & $<0.35$ & $<0.30$ & $<0.30$ & $<0.30$ & $<0.30$ & $<0.40$ & $<0.32$ \\
\hline $\mathrm{T} 1$ & $<0.15$ & & -- & $<0.15$ & $<0.52$ & $<0.15$ & $<0.52$ & & (f) \\
\hline$W(g)$ & $<0.30$ & $<0.40$ & $<0.35$ & $<0.30$ & 0.38 & $<0.30$ & $<0.30$ & & $<0.32$ \\
\hline $2 r$ & 0.15 & $<0.01$ & $<0.08$ & 7.50 & 7.50 & 7.50 & 7.50 & 0.20 & 6.04 \\
\hline $\mathrm{Nd}$ & $<0.40$ & $<0.20$ & $<0.30$ & $<0.40$ & $<0.40$ & $<0.40$ & $<0.40$ & $<0,20$ & $<0.36$ \\
\hline
\end{tabular}

(a) Unless otherwise specified, all analyses were obtained on preconcentrated samples (20 to $50 \mathrm{mg}$ ) by a standard emission spectrographic technique. A 10 to $25 \mathrm{~g}$ sample was obtained for each $10 \mathrm{~g}$ by combining cores from several different locations. Samples were preconcentrated by ashing 1 n still air at $2750^{\circ} \mathrm{C}$. The symbols $<$ and $>$ mean less than and greater than the sensitivity of the spectrographic procedure used. Results for individual analyses are correct within a factor of $40 \%$ or one standard deviation.

(b) Composite sample (25 g) of cores 1isted for specific $10 \mathrm{~g}$ in Tables B-34 and B-35.

(c) Sample ( $35 \mathrm{~g}$ ) taken from cores 6484-34-3A-L050, -L054, and -L058 (midlength center position).

(d) Includes only values for elements with no appreciable $(<20 \%)$ losses on ashing.

(e) Sample ( $35 \mathrm{~g}$ ) taken from cores 6484-29-3A-L051, -L053, -L055, and -L062 (midlength center position).

(f) Measurements on an ashed spectrographic standard indicate 40 to $100 \% 10$ s of element on ashing.

(g) Measurement on an ashed spectrographic standard indicate $220 \% 1$ loss of element on ashing.

(h) High resolution emission spectrographic analysis on unashed 50-mg sample. 
TABLE B-39

SPARK MASS SPECTROMETRIC IMPURITY ANALYSIS OF H-451 AND TS-1240

[in $\mathrm{ppm}^{(\mathrm{a})}$ ]

\begin{tabular}{|c|c|c|c|c|c|c|}
\hline \multirow[b]{2}{*}{ Element } & \multicolumn{3}{|c|}{$\begin{array}{c}\text { H-451 } \\
\text { GA } \log \text { No. } \\
\text { (GLCC Log No./GLCC Lot No.) }\end{array}$} & \multicolumn{3}{|c|}{$\begin{array}{c}\text { TS-1240 } \\
\text { GA Log No. } \\
\text { (UCC Log No./UCC Lot No.) }\end{array}$} \\
\hline & $\begin{array}{c}6484-34 \\
(198 / 426) \\
\end{array}$ & $\begin{array}{c}6484-40 \\
(155 / 426) \\
\end{array}$ & Mean & $\begin{array}{l}5651-72 \\
(30 / 1)\end{array}$ & $\begin{array}{l}5651-74 \\
(42 / 1) \\
\end{array}$ & Mean \\
\hline $\begin{array}{l}\mathrm{Ag} \\
\mathrm{Ba}\end{array}$ & & & & & & \\
\hline $\begin{array}{l}\mathrm{Ba} \\
\mathrm{Ca}\end{array}$ & 2 & 1 & 1.5 & 2 & 5 & 3.5 \\
\hline Co & 7 & & $<4.0$ & & & \\
\hline $\mathrm{Cu}$ & & & & & & \\
\hline $\begin{array}{l}\mathrm{Eu} \\
\mathrm{Hf}\end{array}$ & & & & & & \\
\hline $\begin{array}{l}\mathrm{HI} \\
\mathrm{K}\end{array}$ & 3 & & $<2.0$ & 3 & 2 & 2.5 \\
\hline Lu & & & & & & \\
\hline $\begin{array}{l}\text { Mo } \\
\text { Ni }\end{array}$ & & & & & & \\
\hline Pr & & & & & & \\
\hline $\mathrm{Sc}$ & & & & & & \\
\hline $\mathrm{Sn}$ & & & & & & \\
\hline $\begin{array}{l}\mathrm{Tb} \\
\mathrm{Tm}\end{array}$ & & & & & & \\
\hline $\begin{array}{l}\mathrm{Tm} \\
\mathrm{Yb}\end{array}$ & & & & & & \\
\hline $\mathrm{Al}$ & 1 & & & & & \\
\hline $\mathrm{Be}$ & & & & & & \\
\hline $\mathrm{Cd}$ & & & & & & \\
\hline $\begin{array}{l}\text { Cr } \\
\text { Dy }\end{array}$ & & & & & & \\
\hline $\mathrm{Fe}$ & 10 & 3 & 6.5 & 1 & 1 & 1.0 \\
\hline Ho & & & & & & \\
\hline $\begin{array}{l}\mathrm{La} \\
\mathrm{Mg}\end{array}$ & 1 & & & & & \\
\hline $\begin{array}{l}\mathrm{Mg} \\
\mathrm{Na}\end{array}$ & 6 & 2 & 4.0 & 6 & 3 & 4.5 \\
\hline $\begin{array}{l}\mathrm{Na} \\
\mathrm{P}\end{array}$ & & & & & 1 & \\
\hline $\mathrm{Rb}$ & & 7 & 8.5 & & & \\
\hline $\mathrm{Si}$ & 10 & & 8.3 & 3 & 1 & 2.0 \\
\hline $\begin{array}{l}\mathrm{Sr} \\
\mathrm{Ti}\end{array}$ & & & & & 1 & \\
\hline $\begin{array}{l}11 \\
V\end{array}$ & & & & & & \\
\hline $\mathrm{Zn}$ & 1 & & & & & \\
\hline B & 1 & 1 & 1.0 & 1 & 1 & 1.0 \\
\hline $\begin{array}{l}\mathrm{Bi} \\
\mathrm{Ce}\end{array}$ & & & & & & \\
\hline Cs & & & & & & \\
\hline Er & & & & & & \\
\hline Gd & & & & & & \\
\hline In & & & & & & \\
\hline $\begin{array}{l}\mathrm{Li} \\
\mathrm{Mn}\end{array}$ & & & & & & \\
\hline $\begin{array}{l}\mathrm{Mn} \\
\mathrm{Nb}\end{array}$ & & & & & & \\
\hline $\mathrm{Pb}$ & & & & & & \\
\hline $\mathrm{Sb}$ & & & & & & \\
\hline Sm & & & & & & \\
\hline $\mathrm{Ta}$ & & & & & & \\
\hline $\begin{array}{l}\text { T1 } \\
\text { W }\end{array}$ & 50 & 1 & 25.5 & & & \\
\hline $\mathrm{Zr}$ & & & & 1 & & \\
\hline Nd & & & 3.5 & & & \\
\hline $\begin{array}{l}\mathrm{S} \\
\mathrm{C} 1\end{array}$ & $\begin{array}{l}6 \\
4\end{array}$ & 2 & 3.0 & 6 & $\begin{array}{l}6 \\
1\end{array}$ & 6.0 \\
\hline & & & & & & \\
\hline
\end{tabular}

(a) Al1 analyses were performed on $225-m g$ samples by Accu-Labs Research, Inc., Wheat All analyses were pertor Ridge, Colorado. All elements not reported are less than $(<)$ t pade on composite samples of measurements is approximately \pm 200 to $300 \%$. All measurements made on composite

(cores) taken from midlength center/end center positions in log: 6484-34-108/-1B-028. 
TABLE B-40

SUMMARY OF LITHIUM ANALYSES FOR H-451

\begin{tabular}{|c|c|c|c|c|c|}
\hline $\begin{array}{l}\text { GA } \log \text { No. } \\
\text { (GLCC } \log \text { No., Lot No.) }\end{array}$ & Sample (Core) No. & $\begin{array}{l}\text { No. of } \\
\text { Samples }\end{array}$ & $\begin{array}{l}\text { Location } \\
\text { In Log }\end{array}$ & $\begin{array}{l}\text { Analysis } \\
\text { Method (a) }\end{array}$ & $\begin{array}{l}\text { Li Content } \\
\quad(\text { ppm })\end{array}$ \\
\hline \multirow[t]{3}{*}{$\begin{array}{l}5651-86 \\
(52,408)\end{array}$} & $\begin{array}{l}5651-86 \text { cores shown } \\
\text { in Table B-35 }\end{array}$ & 4 & MLC, MIE & 1 & $<1.0^{(b)}$ \\
\hline & $\begin{array}{l}\text { Composite of } 5651-86 \\
\text { cores show in Table } \\
\text { B-35 }\end{array}$ & 1 & MLC/MLE & $2^{(c)}$ & $<0.07$ \\
\hline & $\begin{array}{l}\text { Composite of cores } \\
5651-86-3 A-L 001 \\
-3 B-L 011\end{array}$ & 1 & MLC/MLE: & 3 & $<0.02$ \\
\hline \multirow[t]{3}{*}{$\begin{array}{l}6484-33 \\
(92,426)\end{array}$} & $\begin{array}{l}6484-33 \text { cores shown } \\
\text { in Table } B-35\end{array}$ & 8 & $\begin{array}{l}\mathrm{MLC}, \mathrm{MLE}, \\
\mathrm{EC}, \mathrm{EE}\end{array}$ & 1 & $<1.0^{\text {(b) }}$ \\
\hline & $\begin{array}{l}\text { Composites of cores } \\
6484-33-1 A-L 108 \\
-1 B-L 117,03 A-L 152, \\
-3 A-L 158,-3 B-L 167 \\
\text { and }-3 B-L 170 \text { (d) }\end{array}$ & 2 & MLE/EE & 3 & $0.04^{(b)}$ \\
\hline & Same as above ${ }^{(d)}$ & 2 & MLE/EE & 4 & $<0.005^{(b)}$ \\
\hline \multirow[t]{8}{*}{$\begin{array}{l}6484-34 \\
(198,426)\end{array}$} & $\begin{array}{l}6484-34 \text { cores shown } \\
\text { in Table } \mathrm{B}-35\end{array}$ & 8 & $\begin{array}{l}\text { MLC, MLE, } \\
\mathrm{EC}, \mathrm{EE}\end{array}$ & 1 & $<1.0(b)$ \\
\hline & $\begin{array}{l}\text { Composite of cores } \\
6484-34-1 \mathrm{~A}-\mathrm{L} 108, \\
-1 \mathrm{~B}-\mathrm{L} 117,-3 \mathrm{~A}-\mathrm{L} 152 \text {, } \\
-3 \mathrm{~A}-\mathrm{L} 158,-3 \mathrm{~B}-\mathrm{L} 167 \\
\text { and }-3 \mathrm{~B}-\mathrm{L} 170 \text { (d) }\end{array}$ & 1 & MLE/EE & 3 & 0.07 \\
\hline & Same as above ${ }^{(d)}$ & 1 & $M L E / E E$ & 4 & $<0.005$ \\
\hline & $\begin{array}{l}\text { Composite of cores } \\
6484-34-3 A-L 056 \\
-3 B-L 067 \text { (d) }\end{array}$ & 1 & MLC & 3 & 0.074 \\
\hline & Same as above ${ }^{(d)}$ & 1 & $\mathrm{MLC}$ & 4 & $<0.005$ \\
\hline & $\begin{array}{l}\text { Composite of cores } \\
6484-34-3 A-L 155 \\
-3 B-L 164 \text { (d) }\end{array}$ & 1 & MLE & 3 & $<0.01$ \\
\hline & Same as above ${ }^{(\mathrm{d})}$ & 1 & MLE & 4 & $<0.005$ \\
\hline & $\begin{array}{l}\text { Composite of cores } \\
6484-34-3 \mathrm{~B}-001 \\
\text { and }-1 \mathrm{~A}-004\end{array}$ & 1 & MLC/EC & 5 & $<1.0$ \\
\hline \multirow[t]{3}{*}{$\begin{array}{l}6484-40 \\
(155,426)\end{array}$} & $\begin{array}{l}6484-40 \text { cores shown } \\
\text { in Table } B-35\end{array}$ & 8 & MLC, $M L E$, & 1 & $<1.0^{(b)}$ \\
\hline & $6484-40-3 B-L 037$ & 1 & MLC & 3 & $<0.01$ \\
\hline & $\begin{array}{l}\text { Composite of cores } \\
6484-40-3 A-002 \\
-1 A-001\end{array}$ & 1 & $\mathrm{MLC} / \mathrm{EC}$ & 5 & $<1.0$ \\
\hline \multirow[t]{2}{*}{$\begin{array}{l}6484-41 \\
(184,426)\end{array}$} & $\begin{array}{l}6484-41 \text { cores shown } \\
\text { in Table B-35 }\end{array}$ & 8 & $\begin{array}{l}\text { MLC, MLE, } \\
\mathrm{EC}, \mathrm{EE}\end{array}$ & 1 & $<1.0^{(\mathrm{b})}$ \\
\hline & $6484-41-3 \mathrm{~B}-1001$ & 1 & MLC & 3 & $<0.01$ \\
\hline
\end{tabular}

(a) 1. Standard emission spectrographic analysis on $20-\mathrm{mg}$ sample.

2. Standard emission spectrographic analysis on a preconcentrated sample (20 to $50 \mathrm{mg}$ ). Sample concentrated by ashing a $25-\mathrm{g}$ sample in sti11 air at $2750^{\circ} \mathrm{C}$.

3. Analysis by atomic absorption on chemically ashed $10-\mathrm{g}$ sample.

4. Isotope dilution spark mass spectrometric analysis on $10-\mathrm{g}$ sample (performed at Oak Ridge National Laboratory).

5. Spark mass spectrometric analysis on 225-mg sample (performed at Accu-Labs Research, Inc., Wheat Ridge, Colorado).

(b) All samples have this value.

(c) Analysis of ashed spectrographic standard (doped) indicates $>50 \%$ loss on ashing.

(d) Companion samples. 
TABLE B-41

SUMMARY OF LITHIUM ANALYSES FOR TS-1240

\begin{tabular}{|c|c|c|c|c|c|c|}
\hline (UCC $\mathrm{G}$ & $\begin{array}{l}\text { GA Log No. } \\
\text { Log No., Lot No.) }\end{array}$ & Sample (Core) No. & $\begin{array}{l}\text { No: of } \\
\text { Samples }\end{array}$ & $\begin{array}{l}\text { Location } \\
\text { in Log }\end{array}$ & $\begin{array}{l}\text { Analysis } \\
\text { Method }(a)\end{array}$ & $\begin{array}{l}\text { L1 Content } \\
\text { (ppm) }\end{array}$ \\
\hline \multirow{4}{*}{\multicolumn{2}{|c|}{$\begin{array}{l}5651-72 \\
(30,1)\end{array}$}} & $\begin{array}{l}\text { 5651-72-3B-L094, } \\
-1 \mathrm{~B}-\mathrm{L} 043\end{array}$ & 2 & MLC, EC & 1 & $<1.0^{(\mathrm{b})}$ \\
\hline & & $\begin{array}{l}\text { Composite of } 5651-72 \\
\text { cores shown in Table } \\
\text { B-36 }\end{array}$ & 1 & $\begin{array}{l}\text { QLC/QLE/ } \\
E C / E E\end{array}$ & $2^{(c)}$ & 0.07 \\
\hline & & $5651-72-1 \mathrm{~A}-\mathrm{L} 009$ & 1 & EC & 3 & $<0.01$ \\
\hline & & $\begin{array}{l}\text { Composite of cores } \\
5651-72-3 A-L 051 \\
-1 B-L 014\end{array}$ & 1 & $\mathrm{MLC} / \mathrm{EC}$ & 5 & $<1.0$ \\
\hline \multirow{3}{*}{\multicolumn{2}{|c|}{$\begin{array}{l}5651-73 \\
(40,1)\end{array}$}} & $\begin{array}{l}5651-73 \text { cores shown in } \\
\text { in Table B-36 }\end{array}$ & 4 & MLC, MLE & 1 & $<1.0^{(b)}$ \\
\hline & & $\begin{array}{l}\text { Composite of } 5651-73 \\
\text { cores shown in Table } \\
\text { B-36 }\end{array}$ & 1 & MLC/MLE & $2^{(c)}$ & 0.45 \\
\hline & & $\begin{array}{l}\text { Composite of cores } \\
\text { 5651-73-3A-L001, } \\
-3 \mathrm{~B} \sim \mathrm{L} 011\end{array}$ & 1 & MLC/MLE & 3 & 0.02 \\
\hline \multirow{4}{*}{\multicolumn{2}{|c|}{$\begin{array}{l}5651-74 \\
(42,1)\end{array}$}} & $\begin{array}{l}5651-74 \text { cores shown in } \\
\text { Table B-36 }\end{array}$ & 8 & $\begin{array}{l}\text { MLC, MLE, } \\
E C, E E\end{array}$ & 1. & $<1.0^{(\mathrm{b})}$ \\
\hline & & $\begin{array}{l}\text { Composite of } 5651-74 \\
\text { cores shown in Table } \\
\text { B-35 }\end{array}$ & 1 & $\begin{array}{l}\mathrm{MLC} / \mathrm{MLE} / \\
\mathrm{EC} / \mathrm{EE}\end{array}$ & $2^{(c)}$ & 0.04 \\
\hline & & $5651-74-3 \mathrm{~B}-\mathrm{L} 065$ & 1 & MLC & 3 & $<0.01$ \\
\hline & & $\begin{array}{l}\text { Composite of cores } \\
5651-74-3 A-108 \\
-1 B-028\end{array}$ & 1 & $\mathrm{MLC} / \mathrm{EC}$ & 5 & $<1.0$ \\
\hline \multirow{3}{*}{\multicolumn{2}{|c|}{$\begin{array}{l}5651-75 \\
(46,1)\end{array}$}} & $\begin{array}{l}5651-75-3 \mathrm{~B}-\mathrm{L} 094 \\
-1 \mathrm{~B}-\mathrm{L} 043\end{array}$ & 2 & MLC, EC & 1 & $<1.0^{(\mathrm{b})}$ \\
\hline & & $\begin{array}{l}\text { Composite of } 5651-75 \\
\text { cores shown in Table } \\
\text { B-36 }\end{array}$ & 1 & $\begin{array}{l}\text { MLC, MLE, } \\
E C, E E\end{array}$ & $2^{(c)}$ & 0.07 \\
\hline & & $5651-75-3 \mathrm{~A}-\mathrm{L} 057$ & 1 & MLC & 3 & $<0.01$ \\
\hline \multirow{3}{*}{\multicolumn{2}{|c|}{$\begin{array}{l}6484-29 \\
(8-1 S-I D-23,1)\end{array}$}} & $\begin{array}{l}6484-29 \text { cores shown in } \\
\text { Table B-36 }\end{array}$ & 8 & $\begin{array}{l}\text { MLC, MLE, } \\
\mathrm{EC}, \mathrm{EE}\end{array}$ & 1 & $<1.0^{(b)}$ \\
\hline & & $\begin{array}{l}\text { Composite of cores } 6484- \\
29-1 A-L 108,-1 B-L 117, \\
-3 A-L 152,-3 A-L 158, \\
-3 B-L 167, \text { and }-3 B-L 170 \\
\text { (d) }\end{array}$ & 1 & $\mathrm{MLE} / \mathrm{EE}$ & 3 & 0.06 \\
\hline & & & 1 & MLE/EE & 4 & $\leq 0.005$ \\
\hline
\end{tabular}

(a) 1. Standard emission spectrographic analysts on $20-\mathrm{mg}$ sample.

2. Standard emission spectrographic analysis on a preconcentrated sample (20 to $50 \mathrm{mg}$ ). Sample concentrated by ashing a $25-\mathrm{g}$ sample in still air at $2750^{\circ} \mathrm{C}$.

3. Analysis by atomic absorption on chemically ashed $10-\mathrm{g}$ sample.

4. Isotope dilution spark mass spectrometric analysis on 10-g sample (performed at Oak Ridge National Laboratory).

5. Spark mass spectrometr1c analysis on 225-mg sample (performed at Accu-Labs Research, Inc., Wheat Ridge, Colorado).

(b) All samples have this value.

(c) Analysis of ashed spectrographic standard (doped) indicates $>50 \%$ loss on ashing.

(d) Companion samples. 